The LHCb Detector at the LHC

This article has been downloaded from IOPscience. Please scroll down to see the full text article. 2008 JINST 3 S08005

(http://iopscience.iop.org/1748-0221/3/08/S08005)

View the table of contents for this issue, or go to the journal homepage for more

Download details:

IP Address: 158.42.146.48

The article was downloaded on 26/06/2013 at 10:35

Please note that terms and conditions apply. 


\section{The LHCb Detector at the LHC}

\section{The LHCb Collaboration}

ABSTRACT: The LHCb experiment is dedicated to precision measurements of $\mathrm{CP}$ violation and rare decays of B hadrons at the Large Hadron Collider (LHC) at CERN (Geneva). The initial configuration and expected performance of the detector and associated systems, as established by test beam measurements and simulation studies, is described.

KEYWORDS: Large detector systems for particle and astroparticle physics; Particle tracking detectors; Gaseous detectors; Calorimeters; Cherenkov detectors; Particle identification methods; Photon detectors for UV, visible and IR photons; Detector alignment and calibration methods; Detector cooling and thermo-stabilization; Detector design and construction technologies and materials. 


\section{The LHCb Collaboration}

Centro Brasileiro de Pesquisas Físicas (CBPF), Rio de Janeiro, Brazil

A. Augusto Alves Jr., L.M. Andrade Filho, ${ }^{1}$ A.F. Barbosa, I. Bediaga, G. Cernicchiaro, G. Guerrer, H.P. Lima Jr, A.A. Machado, J. Magnin, F. Marujo, J.M. de Miranda, A. Reis, A. Santos, A. Toledo

Instituto de Física - Universidade Federal do Rio de Janeiro (IF-UFRJ), Rio de Janeiro, Brazil

K. Akiba, S. Amato, B. de Paula, L. de Paula, T. da Silva, ${ }^{2}$ M. Gandelman, J.H. Lopes, B. Maréchal, D. Moraes, ${ }^{3}$ E. Polycarpo, F. Rodrigues

Laboratoire d'Annecy-le-Vieux de Physique des Particules (LAPP), Université de Savoie, CNRS/IN2P3, Annecy-le-Vieux, France

J. Ballansat, Y. Bastian, D. Boget, I. De Bonis, V. Coco, P.Y. David, D. Decamp, P. Delebecque, C. Drancourt, N. Dumont-Dayot, C. Girard, B. Lieunard, M.N. Minard, B. Pietrzyk, T. Rambure, G. Rospabe, S. T'Jampens

Laboratoire de Physique Corpusculaire Université Blaise Pascal (LPC), CNRS/IN2P3, Aubière, France

Z. Ajaltouni, G. Bohner, R. Bonnefoy, D. Borras, C. Carloganu, H. Chanal, E. Conte, R. Cornat, M. Crouau, E. Delage, O. Deschamps, ${ }^{49}$ P. Henrard, P. Jacquet, C. Lacan, J. Laubser, J. Lecoq, R. Lefèvre, M. Magne, M. Martemiyanov, ${ }^{51}$ M.-L. Mercier, S. Monteil, V. Niess, P. Perret, G. Reinmuth, A. Robert ${ }^{4}$ S. Suchorski

Centre de Physique des Particules de Marseille, Aix-Marseille Université (CPPM) CNRS/IN2P3, Marseille, France

K. Arnaud, E. Aslanides, J. Babel, ${ }^{5}$ C. Benchouk, ${ }^{6}$ J.-P. Cachemiche, J. Cogan, F. Derue, ${ }^{7}$ B. Dinkespiler, P.-Y. Duval, V. Garonne, ${ }^{8}$ S. Favard, ${ }^{9}$ R. Le Gac, F. Leon, O. Leroy, P.-L. Liotard, ${ }^{* *}$ F. Marin, M. Menouni, P. Ollive, S. Poss, A. Roche, M. Sapunov, L. Tocco, ${ }^{\|}$B. Viaud, ${ }^{10}$ A. Tsaregorodtsev

Laboratoire de l'Accélérateur Linéaire (LAL), Université Paris-Sud, CNRS/IN2P3, Orsay, France

Y. Amhis, G. Barrand, S. Barsuk, C. Beigbeder, R. Beneyton, D. Breton, O. Callot, D. Charlet, B. DŠ'Almagne, O. Duarte, F. Fulda-Quenzer, A. Jacholkowska, ${ }^{11}$ B. Jean-Marie, J. Lefrancois, F. Machefert, P. Robbe, M.-H. Schune,V. Tocut, I. Videau 
Laboratoire de Physique Nucléaire et des Hautes Energies(LPNHE), Universités Paris VI et VII, CNRS/IN2P3, Paris, France

M. Benayoun, P. David, L. Del Buono, G. Gilles

Fakultät Physik, Technische Universität Dortmund, Dortmund, Germany

M. Domke, H. Futterschneider, ${ }^{12}$ Ch. Ilgner, P. Kapusta, ${ }^{12,52}$ M. Kolander, R. Krause, ${ }^{12}$ M. Lieng, M. Nedos, K. Rudloff, S. Schleich, R. Schwierz, ${ }^{12}$ B. Spaan, K. Wacker, K. Warda

\section{Max-Planck-Institute for Nuclear Physics, Heidelberg, Germany}

M. Agari," C. Bauer, D. Baumeister," N. Bulian,,* H.P. Fuchs, W. Fallot-Burghardt," T. Glebe,"

W. Hofmann, K.T. Knöpfle, S. Löchner, ${ }^{13}$ A. Ludwig, F. Maciuc, F. Sanchez Nieto, ${ }^{14}$ M. Schmelling, B. Schwingenheuer, E. Sexauer, ${ }^{\|}$N.J. Smale, ${ }^{15}$ U. Trunk, H. Voss

Physikalisches Institut der Universität Heidelberg, Heidelberg, Germany J. Albrecht, S. Bachmann, J. Blouw, M. Deissenroth, H. Deppe,${ }^{13}$ H.B. Dreis, F. Eisele, T. Haas, S. HansmannMenzemer, ${ }^{\ddagger}$ S. Hennenberger, J. Knopf, M. Moch, ${ }^{\ddagger}$ A. Perieanu, ${ }^{\ddagger}$ S. Rabenecker, A. Rausch, C. Rummel, R. Rusnyak, M. Schiller, ${ }^{\ddagger}$ U. Stange, U. Uwer, M. Walter, R. Ziegler

Università di Bologna and Sezione INFN, Bologna, Italy

G. Avoni, G. Balbi, ${ }^{53}$ F. Bonifazi, D. Bortolotti, A. Carbone, I. D’Antone, D. Galli, ${ }^{53}$ D. Gregori, ${ }^{53}$ I. Lax, U. Marconi, G. Peco, V. Vagnoni, G. Valenti, S. Vecchi

Università di Cagliari and Sezione INFN, Cagliari, Italy

W. Bonivento, A. Cardini, S. Cadeddu, V. DeLeo, C. Deplano, S. Furcas, A. Lai, R. Oldeman, D. Raspino, B. Saitta, N. Serra

Università di Ferrara and Sezione INFN, Ferrara, Italy

W. Baldini, S. Brusa, S. Chiozzi, A. Cotta Ramusino, F. Evangelisti, A. Franconieri, S. Germani, ${ }^{16}$ A. Gianoli, L. Guoming, L. Landi, R. Malaguti, C. Padoan, C. Pennini, M. Savriè, S. Squerzanti, T. Zhao, ${ }^{17}$ M. Zhu

Università di Firenze and Sezione INFN, Firenze, Italy

A. Bizzeti, ${ }^{54}$ G. Graziani, M. Lenti, M. Lenzi, F. Maletta, S. Pennazzi, G. Passaleva, M. Veltri, ${ }^{56}$

\section{Laboratori Nazionali di Frascati dell'INFN, Frascati, Italy}

M. Alfonsi, M. Anelli, A. Balla, A. Battisti, G. Bencivenni, P. Campana, M. Carletti, P. Ciambrone, G. Corradi, E. Dané, ${ }^{77}$ A. DiVirgilio, P. DeSimone, G. Felici, C. Forti, ${ }^{49}$ M. Gatta, G. Lanfranchi, F. Murtas, M. Pistilli, M. Poli Lener, R. Rosellini, M. Santoni, A. Saputi, A. Sarti, A. Sciubba, ${ }^{57}$ A. Zossi

Università di Genova and Sezione INFN, Genova, Italy

M. Ameri, S. Cuneo, F. Fontanelli, V. Gracco, G. Miní, M. Parodi, A. Petrolini, M. Sannino, A. Vinci 
Università di Milano-Bicocca and Sezione INFN, Milano, Italy

M. Alemi, C. Arnaboldi, T. Bellunato, M. Calvi, F. Chignoli, A. De Lucia, G. Galotta, R. Mazza,

C. Matteuzzi, M. Musy, P. Negri, D. Perego, ${ }^{\S}$ G. Pessina

Università di Roma "La Sapienza" and Sezione INFN, Roma, Italy

G. Auriemma, ${ }^{58}$ V. Bocci, A. Buccheri, G. Chiodi, S. Di Marco, F. Iacoangeli, G. Martellotti, R. Nobrega, ${ }^{59}$ A. Pelosi, G. Penso,${ }^{59}$ D. Pinci, W. Rinaldi, A. Rossi, R. Santacesaria, C. Satriano, ${ }^{58}$

Università di Roma "Tor Vergata" and Sezione INFN, Roma, Italy

G. Carboni, M. Iannilli, A. Massafferri Rodrigues, ${ }^{18}$ R. Messi, G. Paoluzzi, G. Sabatino, ${ }^{60}$ E. Santovetti, A. Satta

National Institute for Subatomic Physics, Nikhef, Amsterdam, Netherlands

J. Amoraal, G. van Apeldoorn, ${ }^{* *}$ R. Arink, ${ }^{* *}$ N. van Bakel, ${ }^{19}$ H. Band, Th. Bauer, A. Berkien, M. van Beuzekom, E. Bos, Ch. Bron, ${ }^{* *}$ L. Ceelie, M. Doets, R. van der Eijk, J J.-P. Fransen, P. de Groen, V. Gromov, R. Hierck, ${ }^{\prime}$ J. Homma, B. Hommels, ${ }^{20}$ W. Hoogland, ${ }^{* *}$ E. Jans, F. Jansen, L. Jansen, M. Jaspers, B. Kaan, ${ }^{* *}$ B. Koene, ${ }^{* *}$ J. Koopstra, F. Kroes, ${ }^{* *}$ M. Kraan, J. Langedijk, ${ }^{* *}$ M. Merk, S. Mos, B. Munneke, J. Palacios, A. Papadelis, A. Pellegrino, ${ }^{49}$ O. van Petten, T. du Pree, E. Roeland, W. Ruckstuhl, ${ }^{\dagger}$ A. Schimmel, H. Schuijlenburg, T. Sluijk, J. Spelt, J. Stolte, H. Terrier, N. Tuning, A. Van Lysebetten, P. Vankov, J. Verkooijen, B. Verlaat, W. Vink, H. de Vries, L. Wiggers, G. Ybeles Smit, N. Zaitsev," M. Zupan," A. Zwart

Vrije Universiteit, Amsterdam, Netherlands

J. van den Brand, H.J. Bulten, M. de Jong, T. Ketel, S. Klous, J. Kos, B. M'charek, F. Mul, G. Raven, E. Simioni

Center for High Energy Physics, Tsinghua University (TUHEP), Beijing, People's Republic of China

J. Cheng, G. Dai, Z. Deng, Y. Gao, G. Gong, H. Gong, J. He, L. Hou, J. Li, W. Qian, B. Shao, T. Xue, Z. Yang, M. Zeng

AGH-University of Science and Technology, Cracow, Poland

B. Muryn, K. Ciba, A. Oblakowska-Mucha

Henryk Niewodniczanski Institute of Nuclear Physics Polish Academy of Sciences, Cracow, Poland

J. Blocki, K. Galuszka, L. Hajduk, J. Michalowski, Z. Natkaniec, G. Polok, M. Stodulski, M. Witek

Soltan Institute for Nuclear Studies (SINS), Warsaw, Poland

K. Brzozowski, A. Chlopik, P. Gawor, Z. Guzik, A. Nawrot, A. Srednicki, K. Syryczynski, M. Szczekowski 
Horia Hulubei National Institute for Physics and Nuclear Engineering, IFIN-HH, MagureleBucharest, Romania

D.V. Anghel, A. Cimpean, C. Coca, F. Constantin, P. Cristian, D.D. Dumitru, D.T. Dumitru, G. Giolu, C. Kusko, C. Magureanu, Gh. Mihon, M. Orlandea, C. Pavel, R. Petrescu, S. Popescu, T. Preda, A. Rosca, V.L. Rusu, R. Stoica, S. Stoica, P.D. Tarta

Institute for Nuclear Research (INR), Russian Academy of Science, Moscow, Russia

S. Filippov, Yu. Gavrilov, L. Golyshkin, E. Gushchin, O. Karavichev, V. Klubakov, L. Kravchuk, V. Kutuzov, S. Laptev, S. Popov

\section{Institute for Theoretical and Experimental Physics (ITEP), Moscow, Russia}

A. Aref'ev, B. Bobchenko, V. Dolgoshein, V. Egorychev, A. Golutvin, O. Gushchin, A. Konoplyannikov, ${ }^{49}$ I. Korolko, T. Kvaratskheliya, I. Machikhiliyan, ${ }^{49}$ S. Malyshev, E. Mayatskaya, M. Prokudin, D. Rusinov, V. Rusinov, P. Shatalov, L. Shchutska, E. Tarkovskiy, A. Tayduganov, K. Voronchev, O. Zhiryakova

Budker Institute for Nuclear Physics (INP), Novosibirsk, Russia

A. BobrovA. Bondar, S. Eidelman, A. Kozlinsky, L. Shekhtman

Institute for High Energy Physics (IHEP), Protvino, Russia

K.S. Beloous, R.I. Dzhelyadin, ${ }^{49}$ Yu.V. Gelitsky, Yu.P. Gouz, K.G. Kachnov, A.S. Kobelev, V.D. Matveev, V.P. Novikov, V.F. Obraztsov, A.P. Ostankov, V.I. Romanovsky, V.I. Rykalin, A.P. Soldatov, M.M. Soldatov, E.N. Tchernov, O.P. Yushchenko

\section{Petersburg Nuclear Physics Institute, Gatchina, St-Petersburg, Russia}

B. Bochin, N. Bondar, O. Fedorov, V. Golovtsov, S. Guets, A. Kashchuk, ${ }^{49}$ V. Lazarev, O. Maev, P. Neustroev, N. Sagidova, E. Spiridenkov, S. Volkov, An. Vorobyev, A. Vorobyov

University of Barcelona, Barcelona, Spain

E. Aguilo, S. Bota, M. Calvo, A. Comerma, X. Cano, A. Dieguez, A. Herms, E. Lopez, S. Luengo, ${ }^{61}$ J. Garra, Ll. Garrido, D. Gascon, A. Gaspar de Valenzuela, ${ }^{61}$ C. Gonzalez, R. Graciani, E. Grauges, A. Perez Calero, E. Picatoste, J. Riera, ${ }^{61}$ M. Rosello, ${ }^{61}$ H. Ruiz, X. Vilasis, ${ }^{61}$ X. Xirgu

University of Santiago de Compostela (USC), Santiago de Compostela, Spain

B. Adeva, X. Cid Vidal, D. Martĩnez Santos, D. Esperante Pereira, J.L. Fungueiriño Pazos, A. Gallas Torreira, C. Lois Gómez, A. Pazos Alvarez, E. Pérez Trigo, M. Pló Casasús, C. Rodriguez Cobo, P. Rodríguez Pérez, J.J. Saborido, M. Seco P. Vazquez Regueiro

Ecole Polytechnique Fédérale de Lausanne (EPFL), Lausanne, Switzerland

P. Bartalini, ${ }^{21}$ A. Bay, M.-O. Bettler, F. Blanc, J. Borel, B. Carron, C. Currat, G. Conti, O. Dormond, ${ }^{\|}$Y. Ermoline, ${ }^{22}$ P. Fauland, L. Fernandez, $\|$ R. Frei, G. Gagliardi, ${ }^{23}$ N. Gueissaz,

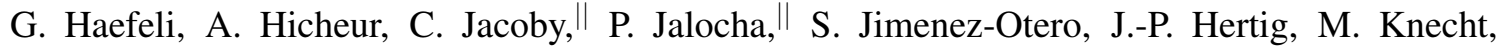
F. Legger, L. Locatelli, J.-R. Moser, M. Needham, L. Nicolas, A. Perrin-Giacomin, J.-P. Perroud, ${ }^{* *}$ C. Potterat, F. Ronga, ${ }^{24}$ O. Schneider, T. Schietinger, ${ }^{25}$ D. Steele, L. Studer, ${ }^{\|}$M. Tareb, M.T. Tran, J. van Hunen," K. Vervink, S. Villa," N. Zwahlen 
University of Zürich, Zürich, Switzerland

R. Bernet, A. Büchler, J. Gassner, F. Lehner, T. Sakhelashvili, C. Salzmann, P. Sievers, S. Steiner, O. Steinkamp, U. Straumann, J. van Tilburg, A. Vollhardt, D. Volyanskyy, M. Ziegler

Institute of Physics and Technologies, Kharkiv, Ukraine

A. Dovbnya, Yu. Ranyuk, I. Shapoval

Institute for Nuclear Research, National Academy of Sciences of Ukraine, KINR, Kiev, Ukraine

M. Borisova, V. Iakovenko, V. Kyva, O. Kovalchuk O. Okhrimenko, V. Pugatch, Yu. Pylypchenko, ${ }^{26}$

H.H. Wills Physics Laboratory, University of Bristol, Bristol, United Kingdom

M. Adinolfi, N.H. Brook, R.D. Head, J.P. Imong, K.A. Lessnoff, F.C.D. Metlica, A.J. Muir, J.H. Rademacker, A. Solomin, P.M. Szczypka

Cavendish Laboratory, University of Cambridge, Cambridge, United Kingdom

C. Barham, C. Buszello, J. Dickens, V. Gibson, S. Haines, K. Harrison, C.R. Jones, S. Katvars, U. Kerzel, C. Lazzeroni, ${ }^{27}$ Y.Y. Li,${ }^{\S}$ G. Rogers, J. Storey, ${ }^{28}$ H. Skottowe, S.A. Wotton

Science and Technology Facilities Council: Rutherford-Appleton Laboratory (RAL), Didcot, United Kingdom

T.J. Adye, C.J. Densham, S. Easo, B. Franek, P. Loveridge, D. Morrow, J.V. Morris, R. Nandakumar, J. Nardulli, A. Papanestis, G.N. Patrick, S. Ricciardi, M.L. Woodward, Z. Zhang

University of Edinburgh, Edinburgh, United Kingdom

R.J.U. Chamonal, P.J. Clark, P. Clarke, S. Eisenhardt, N. Gilardi, A. Khan, ${ }^{29}$ Y.M. Kim, R. Lambert, J. Lawrence, A. Main, J. McCarron, C. Mclean, F. Muheim, A.F. Osorio-Oliveros, S. Playfer, N. Styles, Y. Xie

\section{University of Glasgow, Glasgow, United Kingdom}

A. Bates, L. Carson, F. da Cunha Marinho, F. Doherty, L. Eklund, M. Gersabeck, L. Haddad, A.A. Macgregor, J. Melone, F. McEwan, D.M. Petrie, S.K. Paterson, ${ }^{49, \S}$ C. Parkes, A. Pickford, B. Rakotomiaramanana, E. Rodrigues, A.F. Saavedra, ${ }^{30}$ F.J.P. Soler, ${ }^{62}$ T. Szumlak, S. Viret

\section{Imperial College London, London, United Kingdom}

L. Allebone, O. Awunor, J. Back, ${ }^{31}$ G. Barber, C. Barnes, B. Cameron, D. Clark, I. Clark, P. Dornan, A. Duane, C. Eames, U. Egede, M. Girone, ${ }^{49}$ S. Greenwood, R. Hallam, R. Hare, A. Howard, ${ }^{32}$ S. Jolly, V. Kasey, M. Khaleeq, P. Koppenburg, D. Miller, R. Plackett, D. Price, W. Reece, P. Savage, T. Savidge, B. Simmons, ${ }^{3}$ G. Vidal-Sitjes, D. Websdale 


\section{University of Liverpool, Liverpool, United Kingdom}

A. Affolder, J.S. Anderson, ${ }^{*}$ S.F. Biagi, T.J.V. Bowcock, J.L. Carroll, G. Casse, P. Cooke, S. Donleavy, L. Dwyer, K. Hennessy, ${ }^{*}$ T. Huse, D. Hutchcroft, D. Jones, M. Lockwood, M. McCubbin, R. McNulty, ${ }^{*}$ D. Muskett, A. Noor, G.D. Patel, K. Rinnert, T. Shears, N.A. Smith, G. Southern, I. Stavitski, P. Sutcliffe, M. Tobin, S.M. Traynor, ${ }^{*}$ P. Turner, M. Whitley, M. Wormald, V. Wright

\section{University of Oxford, Oxford, United Kingdom}

J.H. Bibby, S. Brisbane, M. Brock, M. Charles, C. Cioffi, V.V. Gligorov, T. Handford, N. Harnew, F. Harris, M.J.J. John, M. Jones, J. Libby, L. Martin, I.A. McArthur, R. Muresan, C. Newby, B. Ottewell, A. Powell, N. Rotolo, R.S. Senanayake, L. Somerville, A. Soroko, P. Spradlin, P. Sullivan, I. Stokes-Rees, ${ }^{\prime, \S}$ S. Topp-Jorgensen, F. Xing, G. Wilkinson

\section{Physics Department, Syracuse University, Syracuse, N.Y, United States of America}

M. Artuso, I. Belyaev, S. Blusk, G. Lefeuvre, N. Menaa, R. Menaa-Sia, R. Mountain, T. Skwarnicki, S. Stone, J.C. Wang

\section{European Organisation for Nuclear Research (CERN), Geneva, Switzerland}

L. Abadie, G. Aglieri-Rinella, E. Albrecht, J. André,** G. Anelli," N. Arnaud, A. Augustinus, F. Bal, M.C. Barandela Pazos, ${ }^{\text {, }}$ A. Barczyk,${ }^{33}$ M. Bargiotti, J. Batista Lopes, O. Behrendt, S. Berni, P. Binko," V. Bobillier, A. Braem, L. Brarda, J. Buytaert, L. Camilleri, M. Cambpell, G. Castellani, F. Cataneo, ${ }^{* *}$ M. Cattaneo, B. Chadaj, P. Charpentier, S. Cherukuwada, ${ }^{, I}$ E. Chesi, ${ }^{* *}$ J. Christiansen, R. Chytracek, ${ }^{34}$ M. Clemencic, J. Closier, P. Collins, P. Colrain," O. Cooke," B. Corajod, G. Corti, C. D’ Ambrosio, B. Damodaran, ${ }^{\mathbb{I}}{ }^{C}$. David, S. de Capua, G. Decreuse, H. Degaudenzi, H. Dijkstra, J.-P. Droulez, ${ }^{* *}$ D. Duarte Ramos, J.P. Dufey, ${ }^{* *}$ R. Dumps, D. Eckstein, ${ }^{35}$ M. Ferro-Luzzi, F. Fiedler ${ }^{36}$ F. Filthaut, ${ }^{37}$ W. Flegel, ${ }^{* *}$ R. Forty, C. Fournier, M. Frank, C. Frei, B. Gaidioz, C. Gaspar, J.-C. Gayde, P. Gavillet, ${ }^{* *}$ A. Go, ${ }^{38}$ G. Gracia Abril,॥ J.-S. Graulich, ${ }^{39}$ P.-A. Giudici, A. Guirao Elias, P. Guglielmini, T. Gys, F. Hahn, S. Haider, J. Harvey, B. Hay," J.-A. Hernando Morata, J. Herranz Alvarez, E. van Herwijnen, H.J. Hilke, ${ }^{* *}$ G. von Holtey, ${ }^{* *}$ W. Hulsbergen, R. Jacobsson, O. Jamet, C. Joram, B. Jost, N. Kanaya, J. Knaster Refolio, S. Koestner, M. Koratzinos, ${ }^{40}$ R. Kristic, D. Lacarrère, C. Lasseur, T. Lastovicka, ${ }^{41}$ M. Laub, D. Liko, ${ }^{42}$ C. Lippmann, ${ }^{43}$ R. Lindner, M. Losasso, A. Maier, K. Mair, P. Maley, ${ }^{\prime \prime}$ P. Mato Vila, G. Moine, J. Morant, M. Moritz, J. Moscicki, M. Muecke, ${ }^{44}$ H. Mueller, T. Nakada, ${ }^{63}$ N. Neufeld, J. Ocariz ${ }^{45}$ C. Padilla Aranda, U. Parzefall, ${ }^{46}$ M. Patel, M. Pepe-Altarelli, D. Piedigrossi, M. Pivk,, W. Pokorski, S. Ponce, ${ }^{34}$ F. Ranjard, W. Riegler, J. Renaud, ${ }^{* *}$ S. Roiser, A. Rossi, L. Roy, T. Ruf, D. Ruffinoni, ${ }^{* *}$ S. Saladino," A. Sambade Varela, R. Santinelli, S. Schmelling, B. Schmidt, T. Schneider, A. Schöning, ${ }^{24}$ A. Schopper, J. Seguinot, ${ }^{47}$ W. Snoeys, A. Smith, A.C. Smith, ${ }^{\text {II }}$ P. Somogyi, R. Stoica, ${ }^{\mathbb{I}}$ W. Tejessy, ${ }^{* *}$ F. Teubert, E. Thomas, J. Toledo Alarcon, ${ }^{48}$ O. Ullaland, A. Valassi, ${ }^{34}$ P. Vannerem, ${ }^{* *}$ R. Veness, P. Wicht, ${ }^{* *}$ D. Wiedner, W. Witzeling, A. Wright, ${ }^{\prime}$ K. Wyllie, T. Ypsilantis, ${ }^{\dagger}$

\footnotetext{
${ }^{1}$ now at COPPE - Universidade Federal do Rio de Janeiro, COPPE-UFRJ, Rio de Janeiro, Brazil ${ }^{2}$ now at Universidade Federal de Santa Catarina, Florianopolis, Brazil

${ }^{3}$ now at European Organisation for Nuclear Research (CERN), Geneva, Switzerland
} 
${ }^{4}$ now at LPNHE, Université Pierre et Marie Curie, Paris, France

${ }^{5}$ now at Faculté de Sciences Sociales de Toulouse, Toulouse, France

${ }^{6}$ now at Université des Sciences et de la Technologie, Houari Boumediéne, Alger,Algérie

${ }^{7}$ now at Laboratoire de Physique Nucléaire et de Hautes Energies, Paris, France

${ }^{8}$ now at Laboratoire de l'Accélérateur Linéaire, Orsay, France

${ }^{9}$ now at Observatoire de Haute Provence, Saint-Michel de l'Observatoire, France

${ }^{10}$ now at Université de Montréal, Montréal, Canada

${ }^{11}$ now at Laboratoire de Physique Théorique et Astroparticule, Université de Montpellier, Montpellier, France

${ }^{12}$ now at Institut für Kern- und Teilchenphysik, Technische Universität Dresden, Dresden, Germany

${ }^{13}$ now at Gesellschaft für Schwerionenforschung (GSI) Darmstadt, Germany

${ }^{14}$ now at Universitat Autonoma de Barcelona/IFAE, Barcelona, Spain

${ }^{15}$ now at Forschungszentrum Karlsruhe, Eggenstein-Leopoldshafen, Germany

${ }^{16}$ now at Università di Perugia, Perugia, Italy

${ }^{17}$ now at Institute of High Energy Physics (IHEP), Beijing, People's Republic of China

${ }^{18}$ now at Universidade do Estado do Rio de Janeiro (UERJ), Rio de Janeiro, Brazil

${ }^{19}$ now at Stanford University, Palo Alto,United States of America

${ }^{20}$ now at Cavendish Laboratory, University of Cambridge, Cambridge, United Kingdom

${ }^{21}$ now at University of Florida, Gainesville, United States of America

${ }^{22}$ now at Michigan State University, Lansing, United States of America

${ }^{23}$ now at University of Genova and INFN sez. Genova, Genova, Italy

${ }^{24}$ now at Eidgenössische Technische Hochshule Zürich, Zürich, Switzerland

${ }^{25}$ now at Paul Scherrer Institute, Villigen, Switzerland

${ }^{26}$ now at the University of Oslo, Oslo, Norway

${ }^{27}$ now at University of Birmingham, Birmingham, United Kingdom

${ }^{28}$ now at TRIUMF, Vancouver, Canada

${ }^{29}$ now at Brunel University, Uxbridge, United Kingdom

${ }^{30}$ now at University of Sydney, Sydney, Australia

${ }^{31}$ now at University of Warwick, Warwick, United Kingdom

${ }^{32}$ now at University College, London, United Kingdom

${ }^{33}$ now at California Institute of Technology (Caltech),Pasadena, United States of America

${ }^{34}$ now at IT Department, CERN, Geneva, Switzerland

${ }^{35}$ now at Deustches Elektronen-Synchrotron (DESY), Hamburg, Germany

${ }^{36}$ now at Ludwigs-Maximilians University, Munich, Germany

${ }^{37}$ now at Radboud University Nijmegen, Nijmegen, The Netherlands

${ }^{38}$ now at National Center University, Taiwan, Taiwan

${ }^{39}$ now at University of Geneva, Geneva, Switzerland

${ }^{40}$ now at AB Department, CERN, Geneva, Switzerland

${ }^{41}$ now at Oxford University, Oxford, United Kingdom

${ }^{42}$ on leave from Institute of High Energy Physics, Vienna, Austria

${ }^{43}$ now at Gesellschaft für Schwerionenforschung (GSI), Darmstadt, Germany

${ }^{44}$ now at University of Technology, Graz, Austria

${ }^{45}$ now at Université de Paris VI et VII (LPNHE), Paris, France 
${ }^{46}$ now at Albert-Ludwigs-University, Freiburg, Germany

${ }^{47}$ Emeritus, Collège de France, Paris, France

${ }^{48}$ now at Polytechnical University of Valencia, Valencia, Spain

${ }^{49}$ also at CERN, Geneva, Switzerland

${ }^{51}$ also at Institute for Theoretical and Experimental Physics(ITEP), Moscow, Russia

${ }^{52}$ also at Henryk Niewodniczanski Institute of Nuclear Physics, Polish Academy of Sciences, Cracow, Poland

53 also at Alma Mater Studiorum, Università di Bologna, Bologna, Italy

${ }^{54}$ also at University of Modena, Modena, Italy

${ }^{55}$ also at University of Florence, Florence, Italy

${ }^{56}$ also at University of Urbino, Urbino, Italy

57 also at Dipartimento di Energetica, Università di Roma La Sapienza, Roma, Italy

${ }^{58}$ also at University of Basilicata, Potenza, Italy

${ }^{59}$ also at University of Roma "La Sapienza", Rome, Italy

${ }^{60}$ also at LNF, Frascati, Italy

${ }^{61}$ also at Enginyeria i Arquitectura La Salle, Universitat Ramon Llull, Barcelona, Spain

${ }^{62}$ also at Rutherford Appleton Laboratory, Chilton, Didcot, United Kingdom

${ }^{63}$ also at Ecole Polytechnique Fédérale de Lausanne, Lausanne, Switzerland

*from University College Dublin (UCD), Dublin, Ireland

*supported by the Emmy Noether Programme of the Deutsche Forschungsgemeinschaft

${ }^{\S}$ partially supported by the European Community's 5th PCRDT Marie Curie Training Site Programme, at the Centre de Physique des Particules de Marseille, under the Host Fellowship contract HPMT-CT-2001-00339

IIsupported by Marie Curie Early Stage Research Training Fellowship of the European Community's Sixth Framework Programme under contract numbers MEST-CT-2004-007307-MITELCO and MEST-CT-2005-020216-ELACCO

" currently not working in HEP

** retired

$\dagger$ deceased

Corresponding author: Clara Matteuzzi (cxm@mail.cern.ch) 


\section{Contents}

The LHCb Collaboration ii

1 Physics motivations and requirements 1

2 The LHCb Detector 2

2.1 Detector layout 2

2.2 Architecture of the front-end electronics 3

3 The interface to the LHC machine 6

3.1 Beampipe 6

3.1.1 Layout 6

$\begin{array}{ll}3.1 .2 & \text { Vacuum chamber }\end{array}$

3.2 The Beam Conditions Monitor 9

4 Magnet 11

4.1 General description 11

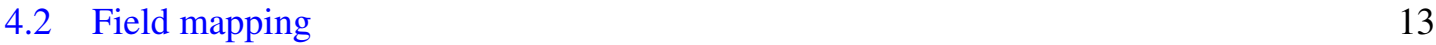

5 Tracking 15

$\begin{array}{llr}5.1 & \text { Vertex locator } & 15\end{array}$

$\begin{array}{lll}\text { 5.1.1 Requirements and constraints } & 16\end{array}$

$\begin{array}{lll}5.1 .2 & \text { Sensors and modules } & 20\end{array}$

5.1.3 Mechanics 25

$\begin{array}{lll}5.1 .4 & \text { Electronics chain } & 28\end{array}$

$\begin{array}{lll}\text { 5.1.5 Material budget } & 33\end{array}$

5.1.6 Test Beam detector commissioning 34

5.1.7 VELO software 36

5.1.8 VELO performance $\quad 40$

5.2 Silicon Tracker 42

5.2.1 Tracker Turicensis $\quad 43$

5.2.2 Inner Tracker 49

$\begin{array}{lll}5.2 .3 & \text { Electronics } & 55\end{array}$

5.2.4 Detector performance $\quad 58$

$\begin{array}{lll}5.3 & \text { Outer Tracker } & 61\end{array}$

5.3.1 Detector layout $\quad 62$

5.3.2 Detector technology $\quad 63$

$\begin{array}{lll}5.3 .3 & \text { Electronics } & 66\end{array}$

$\begin{array}{lll}\text { 5.3.4 Test Beam results } & 69\end{array}$

$\begin{array}{lll}\text { 5.3.5 Alignment and monitoring } & 70\end{array}$

6 Particle identification $\quad 72$ 
$\begin{array}{llr}6.1 & \mathrm{RICH} & 72\end{array}$

$\begin{array}{lll}6.1 .1 & \text { RICH } 1 & 72\end{array}$

$\begin{array}{lll}6.1 .2 & \text { RICH } 2 & 77\end{array}$

$\begin{array}{lll}6.1 .3 & \text { Radiators } & 81\end{array}$

6.1.4 Mirror reflectivity studies $\quad 82$

$\begin{array}{lll}\text { 6.1.5 Photon Detectors } & 83\end{array}$

$\begin{array}{lll}\text { 6.1.6 Electronics } & 87\end{array}$

$\begin{array}{lll}\text { 6.1.7 Monitoring and control } & 90\end{array}$

6.1.8 RICH performance $\quad 93$

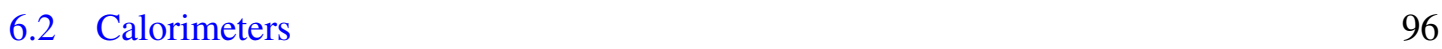

6.2.1 General detector structure $\quad 97$

$\begin{array}{ll}\text { 6.2.2 Electronics overview } & 98\end{array}$

$\begin{array}{lll}\text { 6.2.3 The } \mathrm{pad} / \mathrm{preshower} \text { detector } & 98\end{array}$

6.2.4 The electromagnetic calorimeter 103

$\begin{array}{ll}\text { 6.2.5 The hadron calorimeter } & 107\end{array}$

$\begin{array}{lll}\text { 6.2.6 Electronics of ECAL/HCAL } & 113\end{array}$

6.2.7 Electronics of PS and SPD detectors 118

6.2.8 Monitoring and high voltage system 122

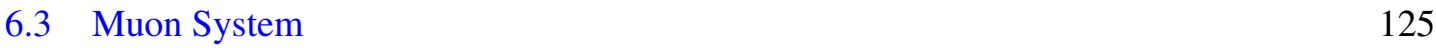

$\begin{array}{lll}6.3 .1 & \text { Overview } & 125\end{array}$

$\begin{array}{ll}\text { 6.3.2 Wire chambers } & 130\end{array}$

$\begin{array}{lll}\text { 6.3.3 } & \text { GEM chambers } & 137\end{array}$

6.3.4 Muon System mechanics 141

6.3.5 Electronics 142

6.3.6 LV and HV systems 146

$\begin{array}{ll}\text { 6.3.7 Experiment control system } & 147\end{array}$

$\begin{array}{lll}\text { 6.3.8 Gas system } & 147\end{array}$

$\begin{array}{lll}\text { 6.3.9 Performance } & 148\end{array}$

7 Trigger $\quad 151$

$\begin{array}{llr}7.1 & \text { Level } 0 \text { trigger } & 153\end{array}$

$\begin{array}{lll}7.1 .1 & \text { Overview } & 153\end{array}$

$\begin{array}{lll}7.1 .2 & \text { Architecture } & 154\end{array}$

$\begin{array}{lll}7.1 .3 & \text { Technology } & 161\end{array}$

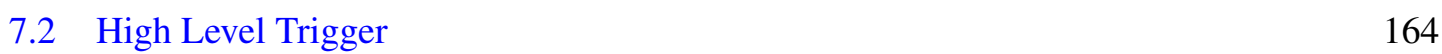

$\begin{array}{llr}7.3 & \text { HLT1 } & 165\end{array}$

$\begin{array}{llr}7.4 & \text { HLT2 } & 166\end{array}$

7.5 HLT monitoring 166

8 Online System $\quad 167$

8.1 System decomposition and architecture 167

$\begin{array}{ll}8.2 & 167\end{array}$

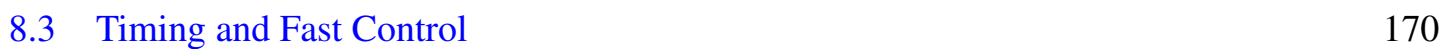


$\begin{array}{lll}8.4 & \text { Experiment Control System } & 171\end{array}$

9 Computing and Resources $\quad 174$

10 Performance 177

$\begin{array}{ll}10.1 \text { Track reconstruction } & 177\end{array}$

\begin{tabular}{lr}
10.2 Particle identification & 180 \\
\hline 10.2 .1 & 181
\end{tabular}

$\begin{array}{ll}\text { 10.2.1 Hadron identification } & 181\end{array}$

10.2.2 Muon identification $\quad 182$

10.2.3 Electron identification $\quad 182$

$\begin{array}{ll}\text { 10.2.4 Photon identification } & 184\end{array}$

$\begin{array}{ll}10.2 .5 \pi^{0} \text { reconstruction } & 184\end{array}$

$\begin{array}{ll}\text { 10.2.6 Expected global performance } & 185\end{array}$

$\begin{array}{ll}11 \text { Summary } & 187\end{array}$

$\begin{array}{ll}\text { Bibliography } & 190\end{array}$ 


\section{Chapter 1}

\section{Physics motivations and requirements}

LHCb is an experiment dedicated to heavy flavour physics at the LHC [1, 2]. Its primary goal is to look for indirect evidence of new physics in $\mathrm{CP}$ violation and rare decays of beauty and charm hadrons.

The current results in heavy flavour physics obtained at the B factories and at the Tevatron are, so far, fully consistent with the CKM mechanism. On the other hand, the level of CP violation in the Standard Model weak interactions cannot explain the amount of matter in the universe. A new source of CP violation beyond the Standard Model is therefore needed to solve this puzzle. With much improved precision, the effect of such a new source might be seen in heavy flavour physics. Many models of new physics indeed produce contributions that change the expectations of the $\mathrm{CP}$ violating phases, rare decay branching fractions, and may generate decay modes which are forbidden in the Standard Model. To examine such possibilities, CP violation and rare decays of $B_{d}, B_{s}$ and $\mathrm{D}$ mesons must be studied with much higher statistics and using many different decay modes.

With the large $b \bar{b}$ production cross section of $\sim 500 \mu \mathrm{b}$ expected at an energy of $14 \mathrm{TeV}$, the LHC will be the most copious source of B mesons in the world. Also $B_{c}$ and b-baryons such as $\Lambda_{b}$ will be produced in large quantities. With a modest luminosity of $2 \times 10^{32} \mathrm{~cm}^{-2} \mathrm{~s}^{-1}$ for $\mathrm{LHCb}, 10^{12} \mathrm{~b} \overline{\mathrm{b}}$ pairs would be produced in $10^{7} \mathrm{~s}$, corresponding to the canonical one year of data taking. Running at the lower luminosity has some advantages: events are dominated by a single pp interaction per bunch crossing (simpler to analyse than those with multiple primary pp interactions), the occupancy in the detector remains low and radiation damage is reduced. The luminosity for the LHCb experiment can be tuned by changing the beam focus at its interaction point independently from the other interaction points. This will allow $\mathrm{LHCb}$ to maintain the optimal luminosity for the experiment for many years from the LHC start-up.

The $\mathrm{LHCb}$ detector must be able to exploit this large number of $\mathrm{b}$ hadrons. This requires an efficient, robust and flexible trigger in order to cope with the harsh hadronic environment. The trigger must be sensitive to many different final states. Excellent vertex and momentum resolution are essential prerequisites for the good proper-time resolution necessary to study the rapidly oscillating $\mathrm{B}_{\mathrm{s}}-\overline{\mathrm{B}}_{\mathrm{s}}$ meson system and also for the good invariant mass resolution, needed to reduce combinatorial background. In addition to electron, muon, $\gamma, \pi^{0}$ and $\eta$ detection, identification of protons, kaons and pions is crucial in order to cleanly reconstruct many hadronic $\mathrm{B}$ meson decay final states such as $\mathrm{B}^{0} \rightarrow \pi^{+} \pi^{-}, \mathrm{B} \rightarrow \mathrm{DK}^{(*)}$ and $\mathrm{B}_{\mathrm{s}} \rightarrow \mathrm{D}_{\mathrm{s}}^{ \pm} \mathrm{K}^{\mp}$. These are key channels for the physics goals of the experiment. Finally, a data acquisition system with high bandwidth and powerful online data processing capability is needed to optimise the data taking. 


\section{Chapter 2}

\section{The LHCb Detector}

\subsection{Detector layout}

$\mathrm{LHCb}$ is a single-arm spectrometer with a forward angular coverage from approximately $10 \mathrm{mrad}$ to 300 (250) mrad in the bending (non-bending) plane. The choice of the detector geometry is justified by the fact that at high energies both the $b$ - and $\bar{b}$-hadrons are predominantly produced in the same forward or backward cone.

The layout of the LHCb spectrometer is shown in figure 2.1. The right-handed coordinate system adopted has the $z$ axis along the beam, and the $y$ axis along the vertical.

Intersection Point 8 of the LHC, previously used by the DELPHI experiment during the LEP

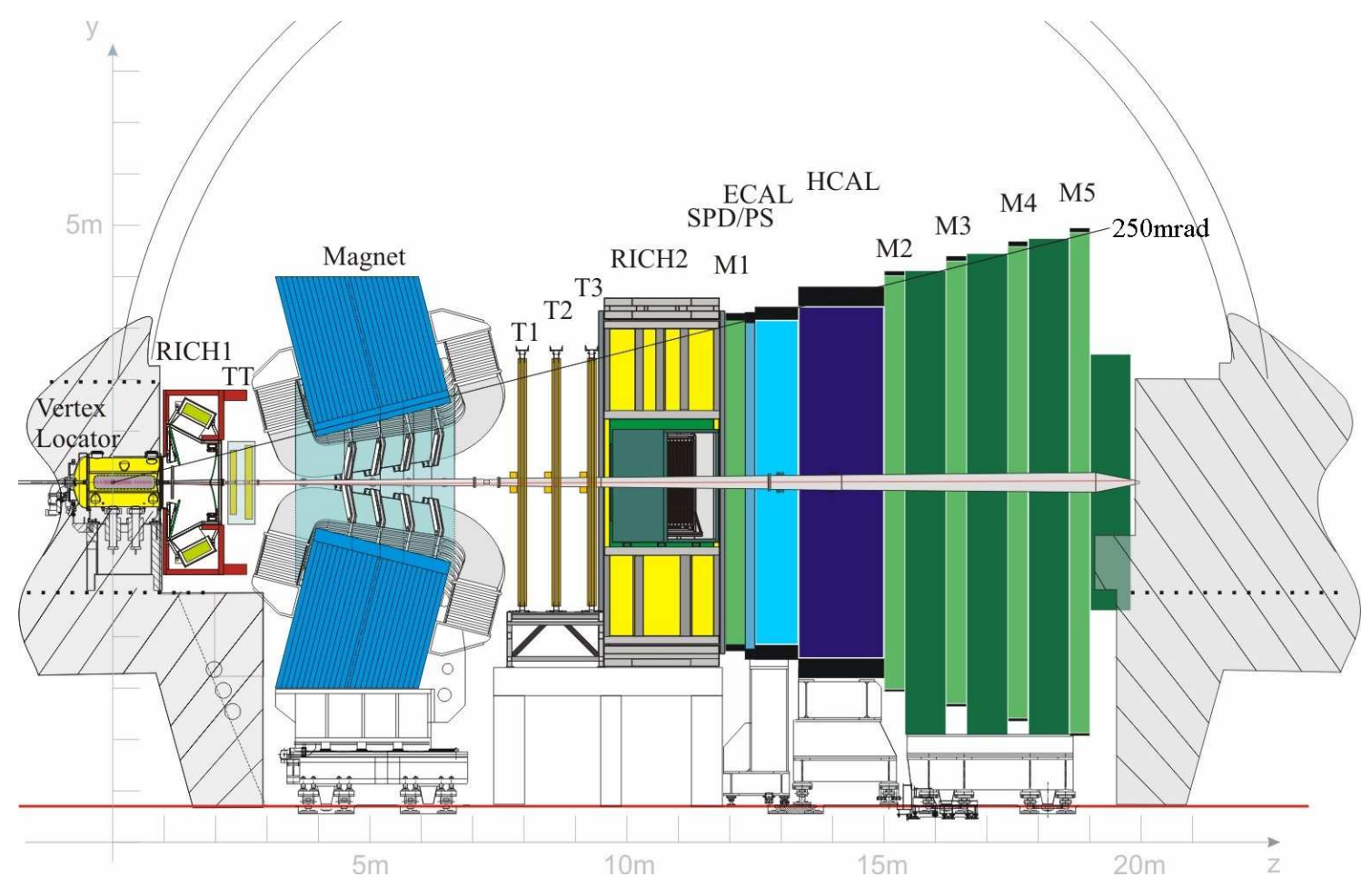

Figure 2.1: View of the $\mathrm{LHCb}$ detector. 
time, has been allocated to the LHCb detector. A modification to the LHC optics, displacing the interaction point by $11.25 \mathrm{~m}$ from the centre, has permitted maximum use to be made of the existing cavern for the $\mathrm{LHCb}$ detector components.

The present paper describes the LHCb experiment, its interface to the machine, the spectrometer magnet, the tracking and the particle identification, as well as the trigger and online systems, including front-end electronics, the data acquisition and the experiment control system. Finally, taking into account the performance of the detectors as deduced from test beam studies, the expected global performance of $\mathrm{LHCb}$, based on detailed MonteCarlo simulations, is summarized.

The interface with the LHC machine is described in section 3. The description of the detector components is made in the following sequence: the spectrometer magnet, a warm dipole magnet providing an integrated field of $4 \mathrm{Tm}$, is described in section 4; the vertex locator system (including a pile-up veto counter), called the VELO, is described in section 5.1; the tracking system made of a Trigger Tracker (a silicon microstrip detector, TT) in front of the spectrometer magnet, and three tracking stations behind the magnet, made of silicon microstrips in the inner parts (IT) and of Kapton/Al straws for the outer parts (OT) is described in sections 5.2 and 5.3; two Ring Imaging Cherencov counters (RICH1 and $\mathrm{RICH} 2$ ) using Aerogel, $\mathrm{C}_{4} \mathrm{~F}_{10}$ and $\mathrm{CF}_{4}$ as radiators, to achieve excellent $\pi$-K separation in the momentum range from 2 to $100 \mathrm{GeV} / \mathrm{c}$, and Hybrid Photon Detectors are described in section 6.1; the calorimeter system composed of a Scintillator Pad Detector and Preshower (SPD/PS), an electromagnetic (shashlik type) calorimeter (ECAL) and a hadronic (Fe and scintillator tiles) calorimeter (HCAL) is described in section 6.2; the muon detection system composed of MWPC (except in the highest rate region, where triple-GEM's are used) is described in section 6.3. The trigger, the online system, the computing resources and the expected performance of the detector are described in sections 7, 8, 9, and 10, respectively.

Most detector subsystems are assembled in two halves, which can be moved out separately horizontally for assembly and maintenance, as well as to provide access to the beampipe.

Interactions in the detector material reduce the detection efficiency for electrons and photons; multiple scattering of pions and kaons complicates the pattern recognition and degrades the momentum resolution. Therefore special attention was paid to the material budget up to the end of the tracking system. Estimations of the material budget of the detector [3] using realistic geometries for the vacuum chamber and all the sub-detectors show that at the end of the tracking, just before entering $\mathrm{RICH} 2$, a particle has seen, on average, about $60 \%$ of a radiation length and about $20 \%$ of an absorption length.

\subsection{Architecture of the front-end electronics}

The front-end architecture chosen for $\mathrm{LHCb}[4,5]$ has to a very large extent been determined by the requirement of making a hardware-based short latency trigger, with an efficient event selection, for complicated B events. A fast first level trigger has been found capable of making an event rate reduction of the order of 1 in 10 . This has for the chosen LHCb luminosity enforced the use of a front-end architecture with a first level trigger rate of up to $1 \mathrm{MHz}$. This was considered to be the highest rate affordable for the data acquisition system (DAQ) and required readout links. The general front-end electronics architecture and data flow in the DAQ interface are shown in figure 2.2. All sub-detectors store sampled detector signals at the $40 \mathrm{MHz}$ bunch crossing rate in 


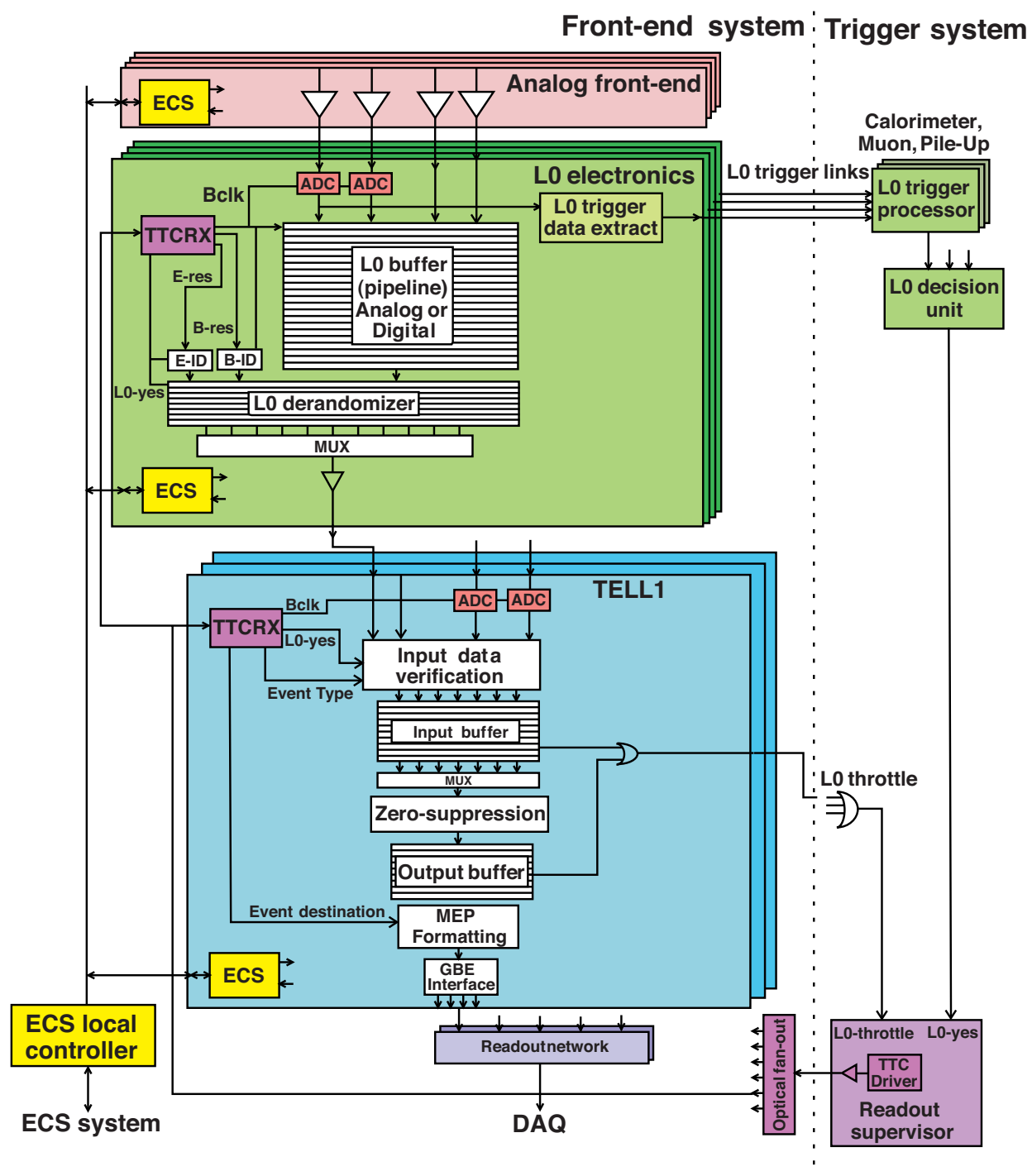

Figure 2.2: General front-end electronics architecture and data flow in the DAQ interface.

$4 \mu$ s deep pipeline buffers, while the hardware-based first level trigger (hereafter called Level-0 or L0) makes the required trigger selection. The speed of the analog detector signal shaping is in general such that only a single sample from one bunch crossing within $25 \mathrm{~ns}$ is extracted from each detector channel when a positive trigger is given (with an exception for the OT with a drift time of up to two bunch crossings). After the first level trigger acceptance, event data are transferred to 16 event deep first level derandomizing buffers to enable a nearly constant data readout rate to the DAQ interface modules located in the counting house. The 16-deep derandomizer allows the frontend to handle closely spaced triggers efficiently. At the output of the derandomizer, data from 32 channels are transferred at a rate of $40 \mathrm{MHz}$, giving a maximum rate of $1.1 \mathrm{MHz}$, and multiplexed into constant data rate links.

All sub-detectors, except the VELO, use optical links (6000 in LHCb) based on the radiation hard GOL (Gigabit Optical Link) serializer chip [6]. A centralized scheme, implemented in the readout supervisor module (see section 8.3), imposes global restrictions on the trigger accept rate 
to prevent overflows of the derandomizer buffers and the following readout and data processing stages. Specific test and monitoring features are integrated into the different front-end systems to assure that thorough testing and monitoring can be made [7] as indicated in figure 2.2.

The Trigger, Timing and Control system (TTC) developed for the LHC experiments [8] is used to distribute clock and sampling phase, timing control (reset and synchronization signals), trigger (trigger accept and trigger types) and a set of dedicated test and calibration commands to all front-end and DAQ interface modules. Control and monitoring of the front-end electronics are based on either the LHCb specific SPECS control interface or the ELMB CAN bus module developed by ATLAS [9].

The reception of accepted event data from the sub-detectors is handled by $3509 \mathrm{U}$ VME sized DAQ interface modules in the counting house. These Field Programmable Gate Array (FPGA) based modules receive front-end data, perform data and event synchronization verifications, appropriate zero-suppression and/or data compression, data buffering and finally send the event information to the DAQ system over up to four Gigabit-Ethernet links per module, as indicated in the lower part of figure 2. The DAQ interface module is in general based on a highly flexible and programmable module named TELL1 [10] with the exception of the RICH detector that has chosen to use a dedicated module ${ }^{1}$ (c.f. section 6.1).

The electronics equipment is located in two different areas. Front-end electronics are installed on the subdetectors or in their close vicinity. Readout and trigger electronics as well as the Experiment Control System and the Data Acquisition system are located in a radiation protected counting house, composed of three levels of electronic barracks separated by a concrete shielding from the experimental area. The control room of the experiment in located on the ground floor.

The present paper does not contain a dedicated chapter with the detailed description of the electronics. The implementation of the specific front-end electronics is described in more detail in the chapters on the individual sub-detectors. Details of the readout supervisor, the Experiment Control System (ECS) and the DAQ system are described as part of the online system (section 8).

\section{Radiation tolerance of the electronics}

All detector components need to tolerate significant radiation doses. Parts of the trigger outside of the counting house are no exception.

The front-end electronics of each sub-detector are located either within the sub-detector itself or on its periphery. Sub-detector specific ASIC's and modules have been custom developed to handle the signal processing needed for the large channel counts in an environment with significant radiation levels. Radiation resistance requirements for all locations with electronics have been defined based on FLUKA simulations with appropriate safety factors [11] (e.g. 10 MRad and $10^{14}$ $1 \mathrm{MeV}$-neutron equivalent $\left(\mathrm{n}_{\mathrm{eq}}\right)$ per $\mathrm{cm}^{2}$ for the front-end chip used for the VELO and the Silicon Tracker; $4 \mathrm{kRad}$ and $10^{12} \mathrm{n}_{\mathrm{eq}} / \mathrm{cm}^{2}$ for the ECAL/HCAL electronics, over 10 years of running). Extensive radiation tests have been made for all electronics components used in the front-end electronics, to verify their correct behaviour, after radiation (total dose and displacement damage), and during radiation exposure (single event upsets).

\footnotetext{
${ }^{1}$ Called UKL1 board.
} 


\section{Chapter 3}

\section{The interface to the LHC machine}

\subsection{Beampipe}

The beampipe design is particularly delicate since the LHCb experiment is focussed on the high rapidity region, where the particle density is high. The number of secondary particles depends on the amount of material seen by incident primary particles. The mass of the beampipe and the presence of flanges and bellows have a direct influence on the occupancy, in particular for the tracking chambers and the RICH detectors. Optimisation of the design and selection of materials were therefore performed in order to maximize transparency in these critical regions [12, 13].

\subsubsection{Layout}

The beampipe, schematically represented in figure 3.1, includes the forward window of the VELO covering the full $\mathrm{LHCb}$ acceptance and four main conical sections, the three closer to the interaction point being made of beryllium and the one further away of stainless steel.

Beryllium was chosen as the material for $12 \mathrm{~m}$ out of the $19 \mathrm{~m}$ long beampipe, for its high transparency to the particles resulting from the collisions. It is the best available material for this application given its high radiation length combined with a modulus of elasticity higher than that of stainless steel. However, its toxicity [14], fragility and cost are drawbacks which had to be taken into account in the design, installation and operation phases. Flanges, bellows and the VELO exit window are made of high strength aluminium alloys which provide a suitable compromise between performance and feasibility. The remaining length, situated outside the critical zone in terms of transparency, is made of stainless steel, a material widely used in vacuum chambers because of its good mechanical and vacuum properties. The VELO window, a spherically shaped thin shell made of aluminium 6061-T6, is $800 \mathrm{~mm}$ in diameter and was machined from a specially forged block down to the final thickness of $2 \mathrm{~mm}$. The machining of the block included a four convolution bellows at its smallest radius. The first beampipe section (UX85/1), that traverses RICH1 and TT (see figure 3.2), is made of $1 \mathrm{~mm}$ thick Be, includes a $25 \mathrm{mrad}$ half-angle cone and the transition to the $10 \mathrm{mrad}$ half-angle cone of the three following beampipe sections. In order to avoid having a flange between the VELO window and UX85/1, the two pieces were electron beam welded before installation. Sections UX85/2 (inside the dipole magnet) and UX85/3 (that traverses the Tracker, RICH2, M1 and part of ECAL) are $10 \mathrm{mrad}$ beryllium cones of wall thickness varying from 1 


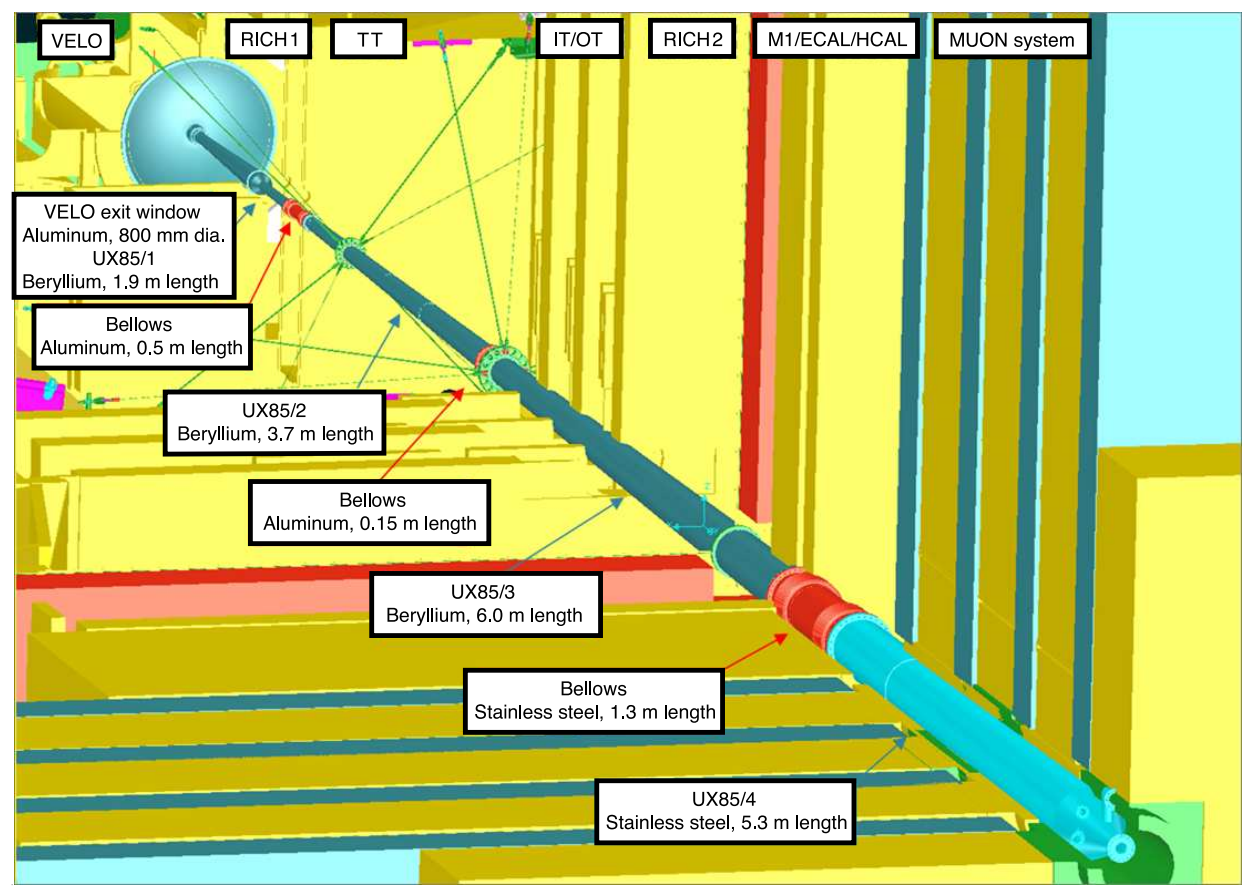

Figure 3.1: The $19 \mathrm{~m}$ long vacuum chamber inside the $\mathrm{LHCb}$ experiment is divided into four main sections. The first three are made of machined beryllium cones assembled by welding and the fourth of stainless steel. Bellows expansion joints provide interconnect flexibility in order to compensate for thermal expansions and mechanical tolerances.

to $2.4 \mathrm{~mm}$ as the diameter increases from 65 up to $262 \mathrm{~mm}$. UX85/3 is connected to a stainless steel bellows through a Conflat seal on the larger diameter. The transition between aluminium and stainless steel is formed using an explosion bonded connection. The three Be beampipes were machined from billets up to $450 \mathrm{~mm}$ long and assembled by arc welding with a non-consumable electrode under inert gas protection (TIG) to achieve the required length. TIG welding was also used to connect the aluminium flanges at the extremities of the tubes.

The UX85/4 section completes the $10 \mathrm{mrad}$ cone and includes a $15^{\circ}$ half-angle conical extremity that provides a smooth transition down to the $60 \mathrm{~mm}$ final aperture. It was manufactured from rolled and welded stainless steel sheet of $4 \mathrm{~mm}$ thickness. A copper coating of $100 \mu \mathrm{m}$ was deposited before assembly on the downstream side end cone to minimise the impedance seen by the beam. The aluminium and stainless steel bellows compensate for thermal expansion during bakeout and provide the necessary flexibility to allow beampipe alignment. Optimised Ultra High Vacuum (UHV) flanges were developed in order to minimise the background contribution from the various connections in the high transparency region [15]. The resulting flange design is based on all-metal Helicoflex seals and high strength AA 2219 aluminium alloy flanges to ensure reliable leak tightness and baking temperatures up to $250^{\circ} \mathrm{C}$. A relatively low sealing force allows the use of aluminium and a significant reduction of the overall mass compared to a standard Conflat flange.

Another important source of background is the beampipe support system [16]. Each beampipe section must be supported at two points, with one fixed, i.e. with displacements restrained in all directions, and the other movable, the latter allowing free displacements along the 


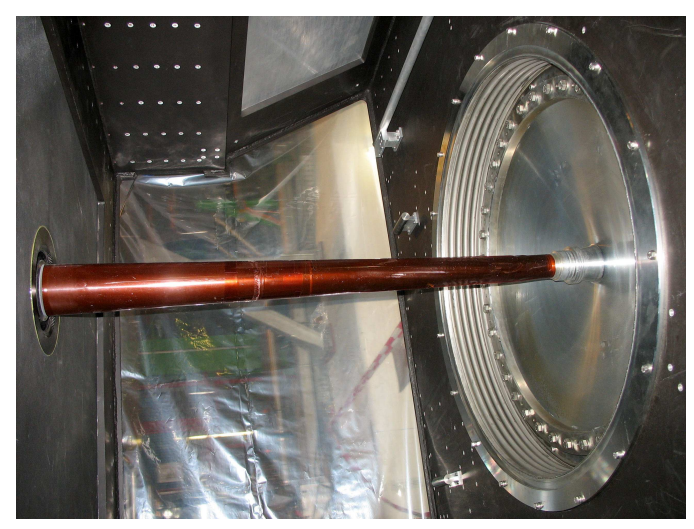

Figure 3.2: View of the VELO exit window and UX85/1 beampipe as installed inside the RICH1 gas enclosure.

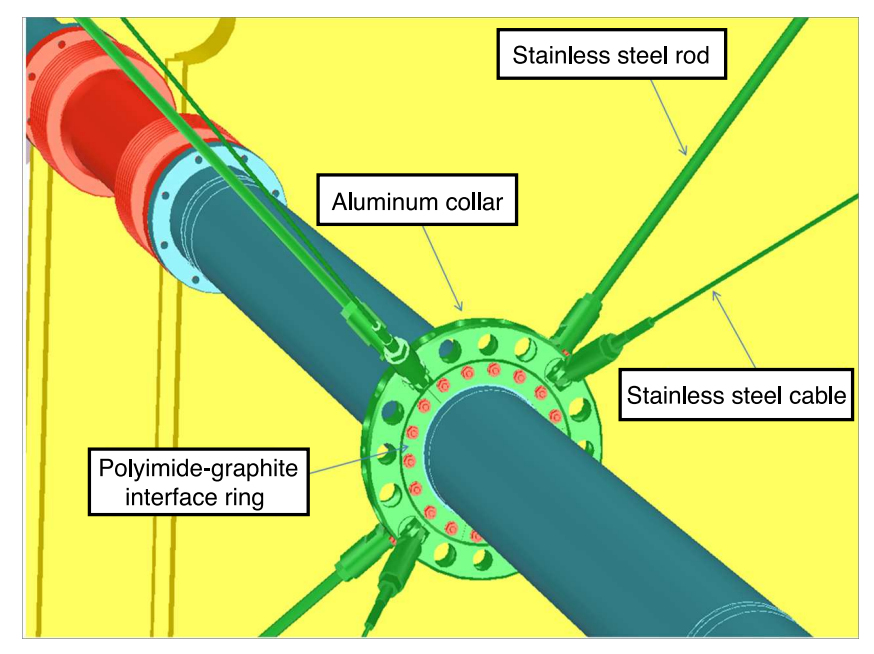

Figure 3.3: Optimised beampipe support inside the acceptance region. A system of eight high resistance cables and rods provide the required rigidity in all directions. A polyimide-graphite ring split in several parts, which are bolted together between the collar and the beampipe, prevents scratches on the beryllium and reduces local stresses at the contact surfaces.

beampipe axis. The fixed supports, which must compensate the unbalanced vacuum forces due to the conical shape of the beampipe, are each constructed using a combination of eight stainless steel cables or rods mounted under tension, pulling in both upstream and downstream directions with an angle to the beam axis (figure 3.3). Where a movable support is required to allow thermal expansion, four stainless steel cables are mounted in the plane perpendicular to the beampipe, blocking all movements except along the beam axis. The support cables and rods are connected to the beampipe through aluminium alloy collars with minimised mass, and an intermediate polyimide-graphite ring to avoid scratching the beryllium and to reduce stresses on contacting surfaces.

The experiment beam vacuum is isolated from the LHC with two sector valves, installed at the cavern entrances, which allow interventions and commissioning independently of the machine vacuum system. 


\subsubsection{Vacuum chamber}

In order to achieve an average total dynamic pressure of $10^{-8}$ to $10^{-9}$ mbar with beam passing through, the LHCb beampipe and the VELO RF-boxes are coated with sputtered non-evaporable getter (NEG) [17]. This works as a distributed pump, providing simultaneously low outgassing and desorption from particle interactions with the walls. Another purpose of the NEG coating is to prevent electron multipacting [18] inside the chamber, since the secondary electron emission yield is much lower than for the chamber material. The UHV pumping system is completed by sputter-ion pumps in the VELO vessel and at the opposite end of the beampipe in order to pump non-getterable gases. Once the NEG coating has been saturated, the chamber must be heated periodically (baked out) to $200^{\circ} \mathrm{C}$, for 24 hours, in order to recover the NEG pumping capacity. The temperature will have to be gradually increased with the number of activation cycles, however it is limited to $250^{\circ} \mathrm{C}$ in the optimised flange assemblies for mechanical reasons. Before NEG activation, the vacuum commissioning procedure also includes the bakeout of the non-coated surfaces inside the VELO vacuum vessel to a temperature of $150^{\circ} \mathrm{C}$. Removable heating jackets are installed during shutdowns covering the VELO window and the beampipe up to the end of RICH2. From there to the end of the muon chambers, a permanent system is installed. As there are no transparency constraints, the insulation of the beampipe inside the muon filters is made from a mixture of silica, metal oxides and glass fiber, whilst the heating is provided by standard resistive tapes.

Such an optimised vacuum chamber must not be submitted to any additional external pressure or shocks while under vacuum, due to the risk of implosion. Hence, it must be vented to atmospheric pressure before certain interventions in the surrounding detectors. Saturation of the NEG coating and consequent reactivation after the venting will be avoided by injecting an inert gas not pumped by the NEG. Neon was found to be the most suitable gas for this purpose because of its low mass and the fact that it is not used as a tracer for leak detection, such as helium or argon. However, commercially available Ne must first be purified before injection. A gas injection system installed in the cavern will provide the clean neon to be injected simultaneously into both VELO beam vacuum and detector vacuum volumes, as the pressure difference between the two volumes must be kept lower than 5 mbar to prevent damage to the VELO RF-boxes (c.f. section 5.1).

\subsection{The Beam Conditions Monitor}

In order to cope with possible adverse LHC beam conditions, particularly with hadronic showers caused by misaligned beams or components performance failures upon particle injection into the LHC, the LHCb experiment is equipped with a Beam Conditions Monitor (BCM) [19]. This system continuously monitors the particle flux at two locations in the close vicinity of the vacuum chamber in order to protect the sensitive LHCb tracking devices. In the case of problems, the BCM system will be the first to respond and will request a dump of the LHC beams. The BCM connects to both the LHCb experiment control system and to the beam interlock controller of the LHC [20]. As a safety system, the BCM is equipped with an uninterruptable power supply and continuously reports its operability also to the vertex locator control system through a hardwired link.

The BCM detectors consist of chemical-vapor deposition (CVD) diamond sensors, which have been proven to withstand radiation doses as high as those that may occur in LHC accident 


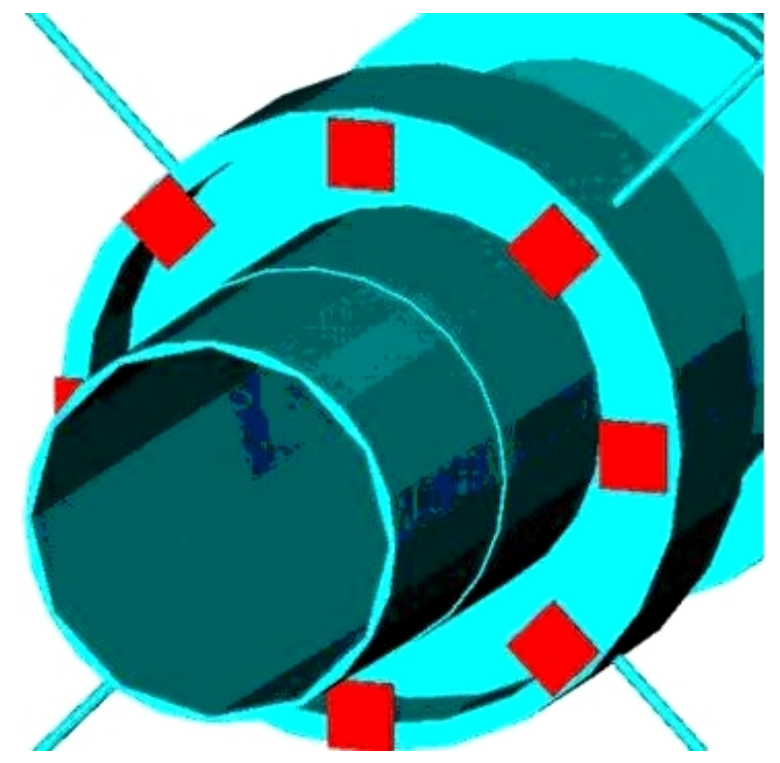

Figure 3.4: Schematic view of the eight CVD-diamond sensors surrounding the beampipe at the downstream BCM station.

scenarios. In order to assure compatibility of the signals with those from other LHC experiments, the dimensions of the sensors are the same as those of the ATLAS and CMS experiments, i.e. their thickness is $500 \mu \mathrm{m}$, the lateral dimensions are $10 \mathrm{~mm} \times 10 \mathrm{~mm}$, with a centered $8 \mathrm{~mm} \times 8 \mathrm{~mm}$ metallized area. The metallization is made of a $500 \AA$ thick gold layer on a $500 \AA$ thick layer of titanium. The radiation resistance of the metallization has been studied with the exposure of a $4 \mathrm{~mm}^{2}$ surface to $4 \times 10^{15}$ protons of an energy of $25 \mathrm{MeV}$ over 18 hours. No sign of degradation was observed.

The two BCM stations are placed at $2131 \mathrm{~mm}$ upstream and $2765 \mathrm{~mm}$ downstream from the interaction point. Each station consists of eight diamond sensors, symmetrically distributed around the vacuum chamber with the sensitive area starting at a radial distance of $50.5 \mathrm{~mm}$ (upstream) and $37.0 \mathrm{~mm}$ (downstream). Figure 3.4 shows the downstream BCM station around the beampipe. The sensors are read out by a current-to-frequency converter card [21] with an integration time of $40 \mu \mathrm{s}$, developed for the Beam Loss Monitors of the LHC.

Simulations were carried out with the GAUSS package [22] to study the expected performance of the BCM. Unstable beam situations are described in a simplified way in generating $7 \mathrm{GeV}$ protons at $3000 \mathrm{~mm}$ upstream of the interaction point in a direction parallel to the beam and in calculating the energy deposited in the BCM sensors caused by these protons. All sensors experience an increase of their signals due to hadronic showers produced by the protons in intermediate material layers. Assuming that during unstable LHC beam condition, the beam comes as close as $475 \mu \mathrm{m}$ (approximately 6 times its RMS) to the RF foil of the VELO (see section 5.1), it would take $40-80 \mu$ s of integration time (or about 20 LHC turns) for the BCM to detect the critical situation and request a beam dump. 


\section{Chapter 4}

\section{Magnet}

\subsection{General description}

A dipole magnet is used in the $\mathrm{LHCb}$ experiment to measure the momentum of charged particles. The measurement covers the forward acceptance of $\pm 250 \mathrm{mrad}$ vertically and of $\pm 300 \mathrm{mrad}$ horizontally. The super-conducting magnet originally proposed in the Technical Proposal [1], would have required unacceptably high investment costs and very long construction time. It was replaced by a warm magnet design with saddle-shaped coils in a window-frame yoke with sloping poles in order to match the required detector acceptance. Details on the design of the magnet are given in the Magnet Technical Design Report [23] and in [24, 25]. The design of the magnet with an integrated magnetic field of $4 \mathrm{Tm}$ for tracks of $10 \mathrm{~m}$ length had to accommodate the contrasting needs for a field level inside the RICHs envelope less than $2 \mathrm{mT}$ and a field as high as possible in the regions between the vertex locator, and the Trigger Tracker tracking station [26]. The design was also driven by the boundary conditions in the experimental hall previously occupied by the DELPHI detector. This implied that the magnet had to be assembled in a temporary position and to be subdivided into two relatively light elements. The DELPHI rail systems and parts of the magnet carriages have been reused as the platform for the LHCb magnet for economic reasons. Plates, $100 \mathrm{~mm}$ thick, of laminated low carbon steel, having a maximum weight of 25 tons, were used to form the identical horizontal bottom and top parts and the two mirror-symmetrical vertical parts (uprights) of the magnet yoke. ${ }^{1}$ The total weight of the yoke is 1500 tons and of the two coils is 54 tons.

The two identical coils are of conical saddle shape and are placed mirror-symmetrically to each other in the magnet yoke. Each coil consists of fifteen pancakes arranged in five triplets and produced of pure Al-99.7 hollow conductor in an annealed state which has a central cooling channel of $25 \mathrm{~mm}$ diameter. The conductor has a specific ohmic resistance below $28 \Omega \cdot \mathrm{m}$ at $20^{\circ} \mathrm{C}$. It is produced in single-length of about $320 \mathrm{~m}$ by rotary extrusion ${ }^{2}$ and tested for leaks with water up to 50 bars and for extrusion imperfections before being wound. The coils were produced in industry ${ }^{3}$ with some equipment and technical support from CERN. Cast Aluminum clamps are used to hold together the triplets making up the coils, and to support and centre the

\footnotetext{
${ }^{1}$ Jebens, Germany.

${ }^{2}$ Holton Machinery, Bournemouth, UK.

${ }^{3}$ SigmaPhi, Vannes, France.
} 


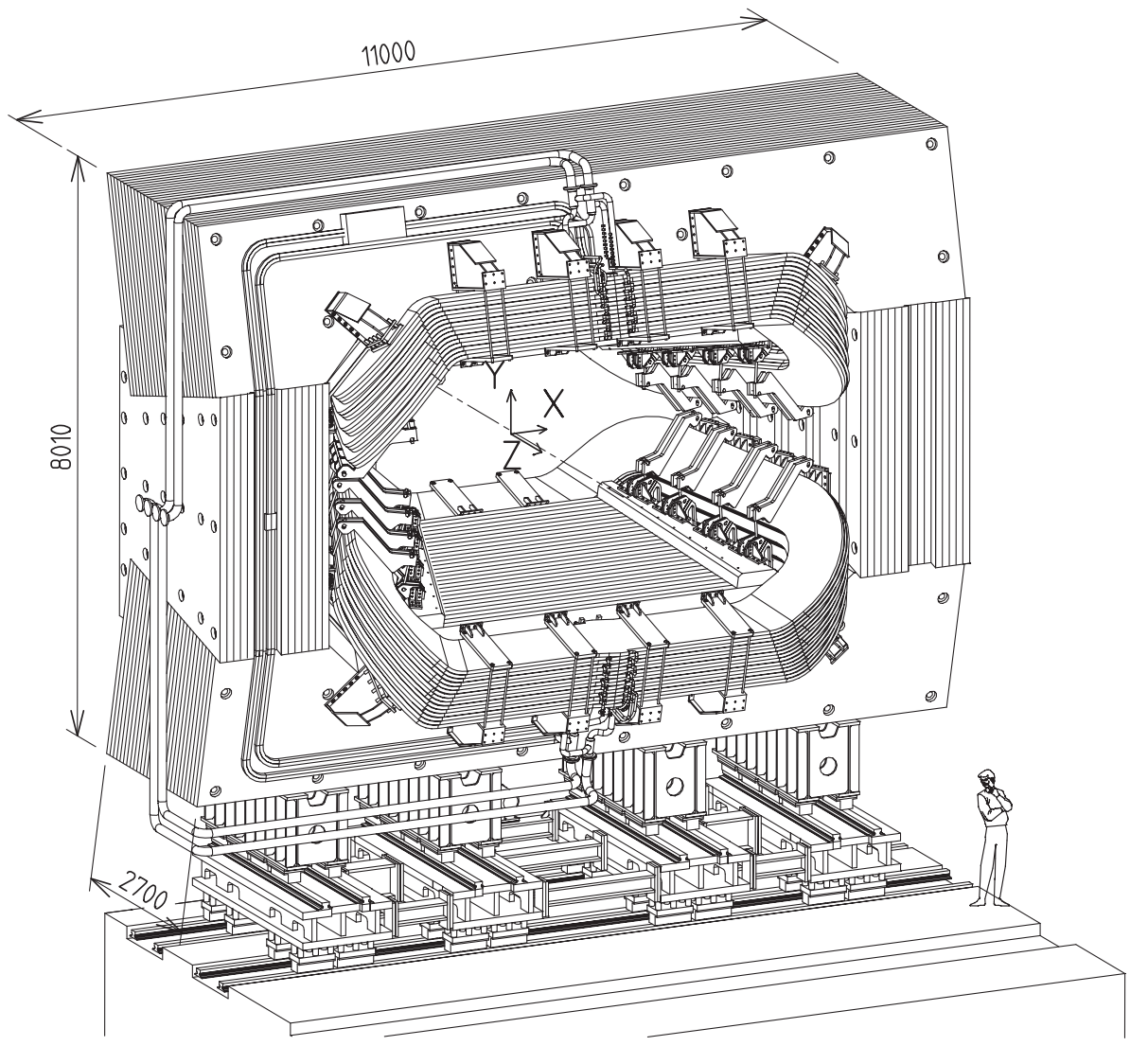

Figure 4.1: Perspective view of the LHCb dipole magnet with its current and water connections (units in $\mathrm{mm}$ ). The interaction point lies behind the magnet.

coils with respect to the measured mechanical axis of the iron poles with tolerances of several millimeters. As the main stress on the conductor is of thermal origin, the design choice was to leave the pancakes of the coils free to slide upon their supports, with only one coil extremity kept fixed on the symmetry axis, against the iron yoke, where electrical and hydraulic terminations are located. Finite element models (TOSCA, ANSYS) have been extensively used to investigate the coils support system with respect to the effect of the electromagnetic and thermal stresses on the conductor, and the measured displacement of the coils during magnet operation matches the predicted value quite well. After rolling the magnet into its nominal position, final precise alignment of the yoke was carried out in order to follow the $3.6 \mathrm{mrad}$ slope of the LHC machine and its beam. The resolution of the alignment measurements was about $0.2 \mathrm{~mm}$ while the magnet could be aligned to its nominal position with a precision of $\pm 2 \mathrm{~mm}$. Details of the measurements of the dipole parameters are given in table 4.1. A perspective view of the magnet is given in figure 4.1.

The magnet is operated via the Magnet Control System that controls the power supply and monitors a number of operational parameters (e.g. temperatures, voltages, water flow, mechanical movements, etc.). A second, fully independent system, the Magnet Safety System (MSS), ensures the safe operation and acts autonomously by enforcing a discharge of the magnet if critical parameters are outside the operating range. The magnet was put into operation and reached its nominal 
Table 4.1: Measured main parameters of the LHCb magnet.

\begin{tabular}{|c|c|}
\hline Non-uniformity of $|B|$ & $\pm 1 \%$ in planes xy of $1 \mathrm{~m}^{2}$ from $\mathrm{z}=3 \mathrm{~m}$ to $\mathrm{z}=8 \mathrm{~m}$ \\
\hline $\int B d l$ upstream TT region $(0-2.5 \mathrm{~m})$ & $0.1159 \mathrm{Tm}$ \\
\hline $\int B d l$ downstream TT region $(2.5-7.95 \mathrm{~m})$ & $3.615 \mathrm{Tm}$ \\
\hline Max field at HPD's of RICH1 & $20 \times 10^{-4} \mathrm{~T}\left(14 \times 10^{-4} \mathrm{~T}\right.$ with mu-metal $)$ \\
\hline Max field at HPD's of RICH2 & $9 \times 10^{-4} \mathrm{~T}$ \\
\hline Electric power dissipation & $4.2 \mathrm{MW}$ \\
\hline Inductance L & $1.3 \mathrm{H}$ \\
\hline Nominal / maximum current in conductor & $5.85 \mathrm{kA} / 6.6 \mathrm{kA}$ \\
\hline Total resistance (two coils + bus bars) & $\mathrm{R}=130 \mathrm{~m} \Omega$ @ $20^{\circ} \mathrm{C}$ \\
\hline Total voltage drop (two coils) & $730 \mathrm{~V}$ \\
\hline Total number of turns & $2 \times 225$ \\
\hline Total water flow & $150 \mathrm{~m}^{3} / \mathrm{h}$ \\
\hline Water Pressure drop & $11 \mathrm{bar} @ \Delta \mathrm{T}=25^{\circ} \mathrm{C}$ \\
\hline Overall dimensions H $\mathrm{V} \mathrm{x} \mathrm{L}$ & $11 \mathrm{~m} \mathrm{x} 8 \mathrm{~m} \mathrm{x} 5 \mathrm{~m}$ \\
\hline Total weight & 1600 tons \\
\hline
\end{tabular}

current of $5.85 \mathrm{kA}$ in November 2004, thereby being the first magnet of the LHC experiments operational in the underground experimental areas. Several magnetic field measurement campaigns have been carried out during which the magnet has shown stable and reliable performance.

\subsection{Field mapping}

In order to achieve the required momentum resolution for charged particles, the magnetic field integral $\int B d l$ must be measured with a relative precision of a few times $10^{-4}$ and the position of the B-field peak with a precision of a few millimetres. A semi-automatic measuring device was constructed which allowed remotely controlled scanning along the longitudinal axis of the dipole by means of an array of Hall probes. The measuring machine was aligned with a precision of $1 \mathrm{~mm}$ with respect to the experiment reference frame. The support carrying the Hall probes could be manually positioned in the horizontal and vertical direction such as to cover the magnetic field volume of interest. The Hall probe array consisted of 60 sensor cards mounted on a G10 support covering a grid of $80 \mathrm{~mm} \times 80 \mathrm{~mm}$. Each sensor card contained three Hall probes mounted orthogonally on a cube together with a temperature sensor and the electronics required for remote readout. These 3D sensor cards ${ }^{4}$ have been calibrated to a precision of $10^{-4}$ using a rotating setup in an homogeneous field together with an NMR for absolute field calibration [27]. The calibration process allowed correcting for non-linearity, temperature dependence and non-orthogonal mounting of the Hall probes.

The goal of the field mapping campaigns was to measure the three components of the magnetic field inside the tracking volume of the detector for both magnet polarities and to compare it to the magnetic field calculations obtained with TOSCA. ${ }^{5}$ For the measurement of $\mathrm{CP}$ asymmetries it

\footnotetext{
${ }^{4}$ Developed in collaboration between CERN and NIKHEF for the ATLAS muon system.

${ }^{5}$ Vector Field TM.
} 


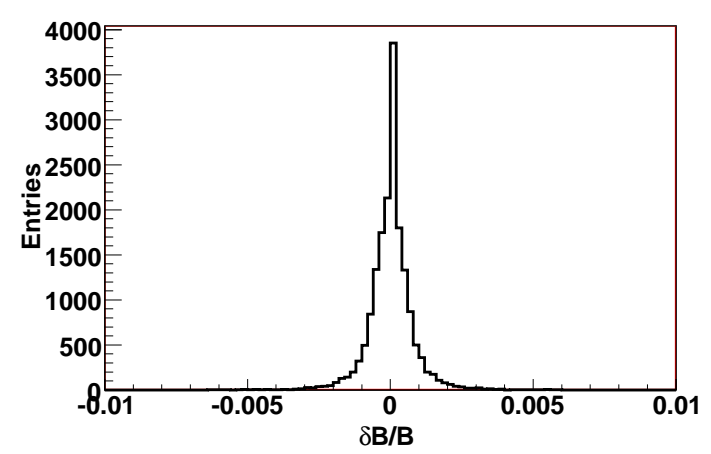

Figure 4.2: Relative difference between the measurements of B using different Hall probes at the same position in the magnet. The resolution is completely dominated by the precision of the calibration of the Hall probes.

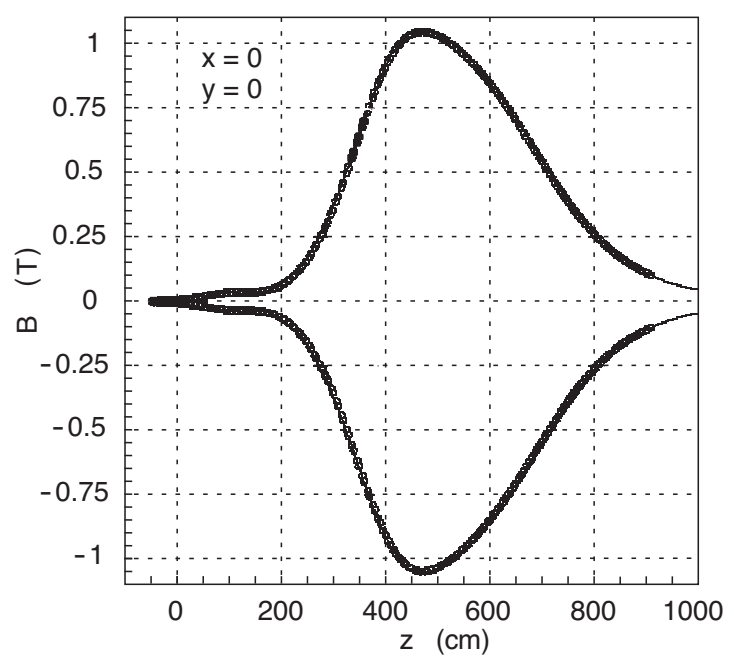

Figure 4.3: Magnetic field along the $z$ axis.

is important to control the systematic effects of the detector, by changing periodically the direction of the magnetic field. To this purpose, the impact of hysteresis effects on the reproducibility of the magnetic field has to be taken into account.

The magnetic field has been measured in the complete tracking volume inside the magnet and in the region of the VELO and the tracking stations, and also inside the magnetic shielding for the RICH1 and RICH2 photon detectors. The precision of the measurement obtained for the field mapping in the tracking volume is about $4 \times 10^{-4}$, as shown in figure 4.2. The main component, $\mathrm{B}_{y}$, is shown in figure 4.3 for both polarities, together with the result of the model calculation. The overall agreement is excellent; however, in the upstream region of the detector (VELO, RICH1) a discrepancy of about 3.5\% for the field integral has been found which can be attributed both to the precision of the TOSCA model computation and to the vicinity of the massive iron reinforcement embedded in the concrete of the hall. In all other regions the agreement between measurement and calculation is better than $1 \%$.

In conclusion, the three components of the magnetic field have been measured with a fine grid of $8 \times 8 \times 10 \mathrm{~cm}^{3}$ spanning from the interaction point to the RICH2 detector (i.e. over distance of about $9 \mathrm{~m}$ ) and covering most of the LHCb acceptance region. The precision of the field map obtained is about $4 \times 10^{-4}$ and the absolute field value is reproducible for both polarities to better than this value, provided the right procedure for the demagnetization of the iron yoke is applied. 


\section{Chapter 5}

\section{Tracking}

The LHCb tracking system consists of the vertex locator system (VELO) and four planar tracking stations: the Tracker Turicensis (TT) upstream of the dipole magnet and T1-T3 downstream of the magnet. VELO and TT use silicon microstrip detectors. In T1-T3, silicon microstrips are used in the region close to the beam pipe (Inner Tracker, IT) whereas straw-tubes are employed in the outer region of the stations (Outer Tracker, OT). The TT and the IT were developed in a common project called the Silicon Tracker (ST).

The VELO is described in section 5.1 the ST in section 5.2 and the OT in section 5.3.

\subsection{Vertex locator}

The VErtex LOcator (VELO) provides precise measurements of track coordinates close to the interaction region, which are used to identify the displaced secondary vertices which are a distinctive feature of $b$ and c-hadron decays [28]. The VELO consists of a series of silicon modules, each providing a measure of the $r$ and $\phi$ coordinates, arranged along the beam direction (figure 5.1). Two planes perpendicular to the beam line and located upstream of the VELO sensors are called the pile-up veto system and are described in section 7.1. The VELO sensors are placed at a radial distance from the beam which is smaller than the aperture required by the LHC during injection and must therefore be retractable. The detectors are mounted in a vessel that maintains vacuum around the sensors and is separated from the machine vacuum by a thin walled corrugated aluminum sheet. This is done to minimize the material traversed by a charged particle before it crosses the sensors and the geometry is such that it allows the two halves of the VELO to overlap when in the closed position. Figure 5.2 shows a cross section of the VELO vessel, illustrating the separation between the primary (beam) vacuum and the secondary (detector) vacuum enclosed by the VELO boxes. Figure 5.3 shows an expanded view from inside one of the boxes, with the sides cut away to show the staggered and overlapping modules of the opposite detector half. The corrugated foils, hereafter referred to as RF-foils, form the inner faces of the boxes (RF-boxes) within which the modules are housed. They provide a number of functions which are discussed in the following sections. 


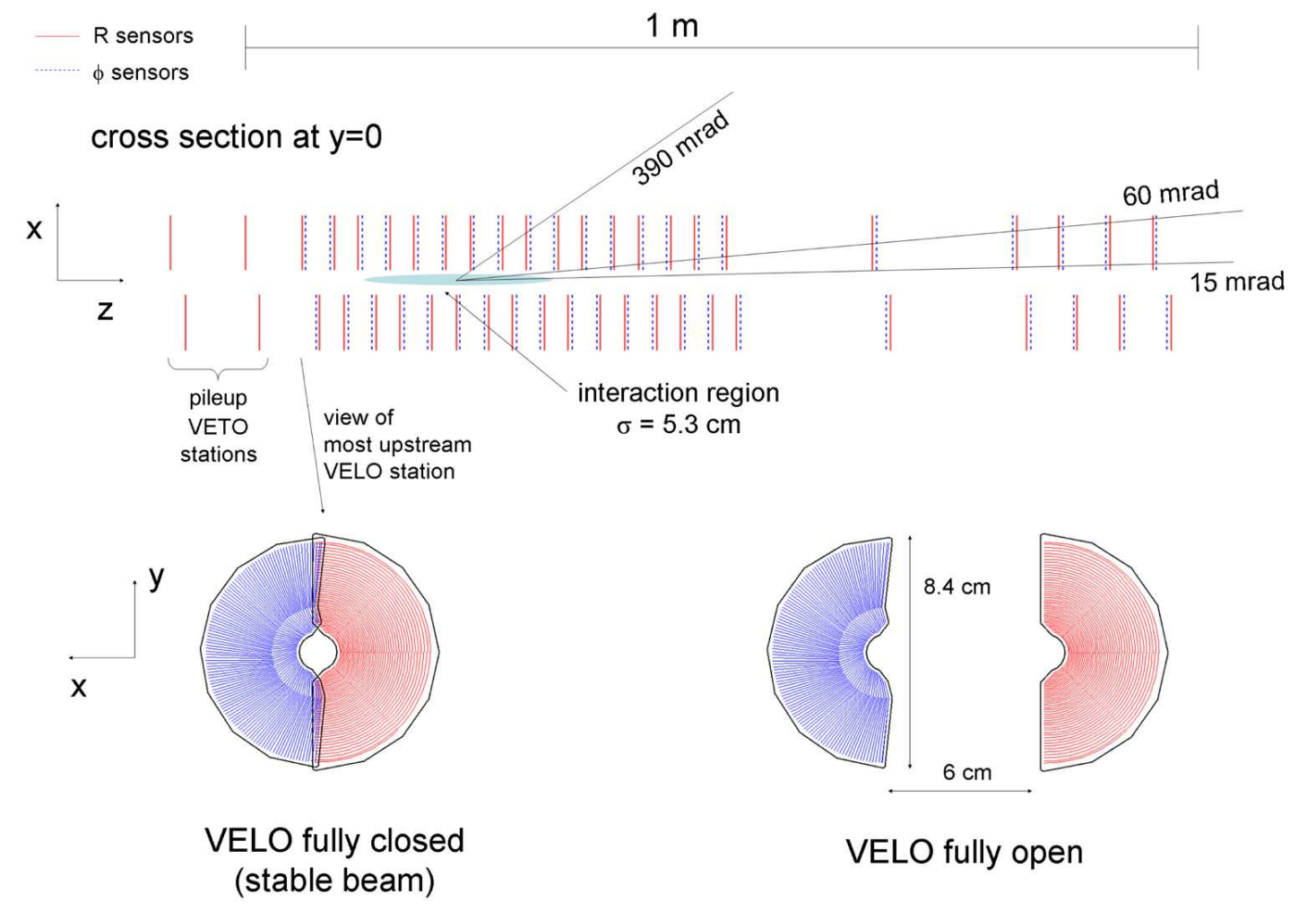

Figure 5.1: Cross section in the $(x, z)$ plane of the VELO silicon sensors, at $y=0$, with the detector in the fully closed position. The front face of the first modules is also illustrated in both the closed and open positions. The two pile-up veto stations are located upstream of the VELO sensors.

\subsubsection{Requirements and constraints}

The ability to reconstruct vertices is fundamental for the LHCb experiment. The track coordinates provided by the VELO are used to reconstruct production and decay vertices of beauty- and charmhadrons, to provide an accurate measurement of their decay lifetimes and to measure the impact parameter of particles used to tag their flavour. Detached vertices play a vital role in the High Level Trigger (HLT, see section 7.2), and are used to enrich the b-hadron content of the data written to tape, as well as in the LHCb off-line analysis. The global performance requirements of the detector can be characterised with the following interrelated criteria:

- Signal to noise ${ }^{1}$ ratio $(\mathrm{S} / \mathrm{N})$ : in order to ensure efficient trigger performance, the VELO aimed for an initial signal to noise ratio of greater than 14 [29].

- Efficiency: the overall channel efficiency was required to be at least $99 \%$ for a signal to noise cut $\mathrm{S} / \mathrm{N}>5$ (giving about 200 noise hits per event in the whole VELO detector).

\footnotetext{
${ }^{1}$ Signal $\mathrm{S}$ is defined as the most probable value of a cluster due to a minimum-ionizing particle and noise $\mathrm{N}$ as the RMS value of an individual channel.
} 


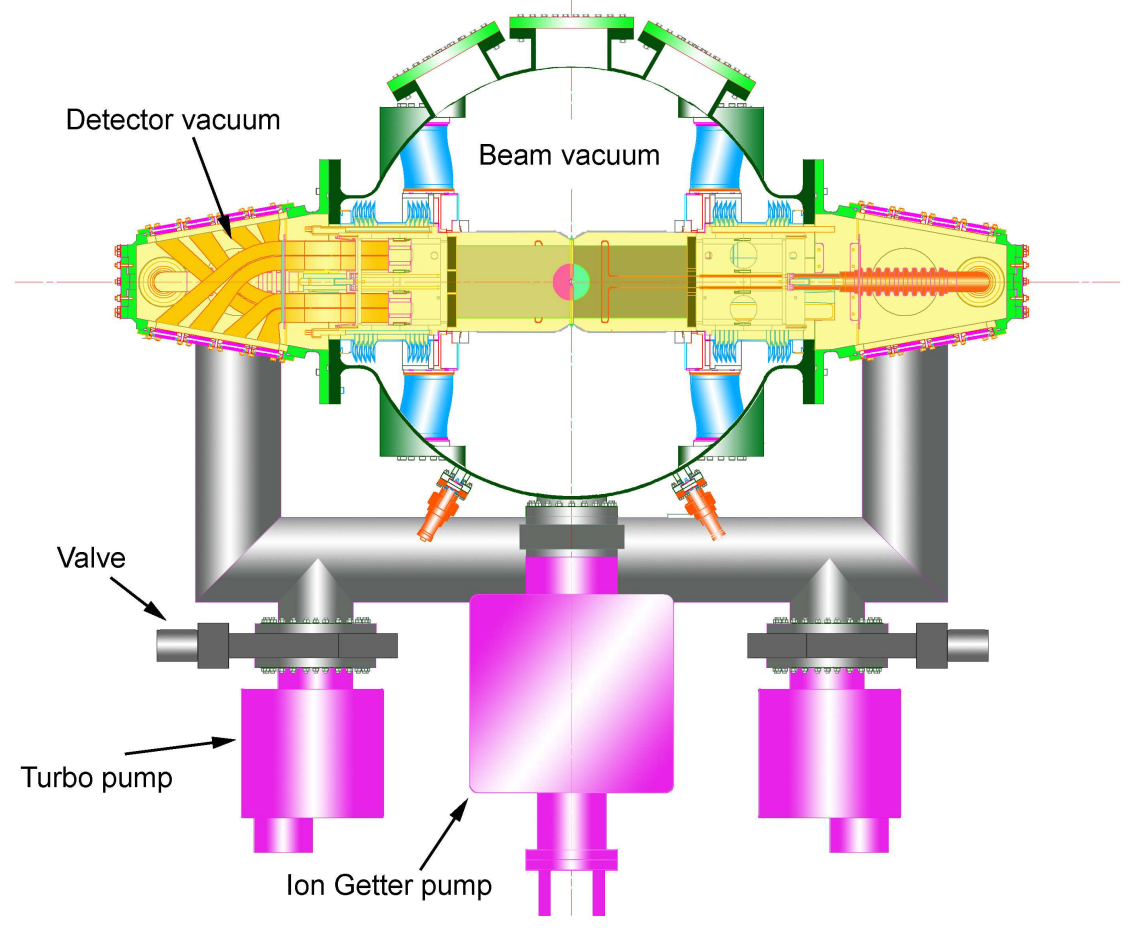

Figure 5.2: Cross section of the VELO vacuum vessel, with the detectors in the fully closed position. The routing of the signals via kapton cables to vacuum feedthroughs are illustrated. The separation between the beam and detector vacua is achieved with thin walled aluminium boxes enclosing each half.

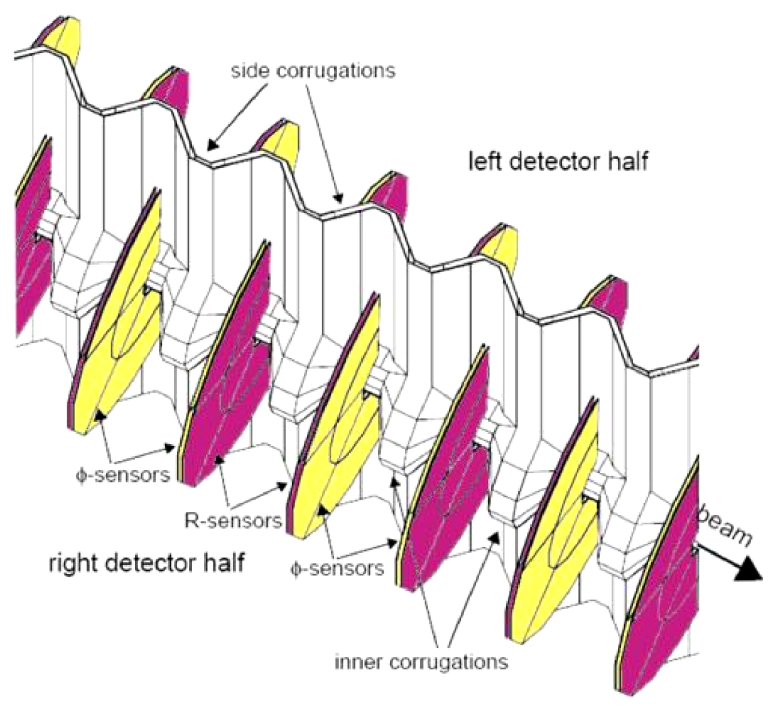

Figure 5.3: Zoom on the inside of an RF-foil, as modelled in GEANT, with the detector halves in the fully closed position. The edges of the box are cut away to show the overlap with the staggered opposing half. The R- and $\phi$-sensors are illustrated with alternate shading. 
- Resolution: a spatial cluster resolution of about $4 \mu \mathrm{m}$ was aimed at for $100 \mathrm{mrad}$ tracks in the smallest strip pitch region (about $40 \mu \mathrm{m}$ ), in order to achieve the impact parameter resolution performance described in section 10. Furthermore, it was required that the resolution not be degraded by irradiation nor by any aspect of the sensor design.

Another important consideration is the spillover probability, which is defined as the fraction of the peak signal remaining after $25 \mathrm{~ns}$. An additional requirement imposed on the system, affecting the readout electronics, is that the spillover probability be less than 0.3 , in order to keep the number of remnant hits at a level acceptable for the HLT [30].

The construction of the VELO followed a number of requirements and constraints, which are briefly described in this section.

\section{Geometrical}

The VELO has to cover the angular acceptance of the downstream detectors, i.e. detect particles with a pseudorapidity in the range ${ }^{2} 1.6<\eta<4.9$ and emerging from primary vertices in the range $|z|<10.6 \mathrm{~cm}$. The detector setup was further constrained by the following considerations:

- Polar angle coverage down to $15 \mathrm{mrad}$ for a track emerging at $z=10.6 \mathrm{~cm}$ downstream from the nominal interaction point (IP), together with the minimum distance of the sensitive area to the beam axis ( $8 \mathrm{~mm}$, see below), and the requirement that a track should cross at least three VELO stations, defined the position $z_{N-2}$ of the first of the three most downstream stations: $z_{N-2} \simeq 65 \mathrm{~cm}$.

- A track in the LHCb spectrometer angular acceptance of $300 \mathrm{mrad}$ should cross at least three VELO stations. Given a maximum ${ }^{3}$ outer radius of the sensors of about $42 \mathrm{~mm}$, the distance between stations in the central region needed to be smaller than $5 \mathrm{~cm}$. Requiring four stations to be traversed (or allowing for missing hits in one of four stations), imposed a module pitch of at most $3.5 \mathrm{~cm}$. Dense packing of stations near the IP also reduces the average extrapolation distance from the first measured hit to the vertex.

- For covering the full azimuthal acceptance and for alignment issues, the two detector halves were required to overlap. This was achieved by shifting along $z$ the positions of sensors in one half by $1.5 \mathrm{~cm}$ relative to sensors in the opposite half.

The use of cylindrical geometry ( $r \phi$ coordinates), rather than a simpler rectilinear scheme, was chosen in order to enable fast reconstruction of tracks and vertices in the LHCb trigger. Indeed, simulations showed that 2D $(r z)$ tracking allows a fast reconstruction in the HLT with sufficient impact parameter resolution to efficiently select events with b-hadrons. For this reason, an $r \phi$ geometry was selected for the design. Each VELO module was designed to provide the necessary 3D spatial information to reconstruct the tracks and vertices. One of the two sensors of the module, called the $\phi$-measuring sensor, or $\phi$-sensor, provides information on the azimuthal coordinate

\footnotetext{
${ }^{2}$ Some coverage of negative pseudorapidity is used to improve the primary vertex reconstruction and, using two special stations, to reduce the number of multiple-interaction events passing the Level-0 trigger (L0, see section 7.1).

${ }^{3}$ This allowed the use of $10 \mathrm{~cm} \mathrm{Si} \mathrm{wafers} \mathrm{for} \mathrm{sensor} \mathrm{production.}$
} 
around the beam. The other sensor, called the $r$-measuring sensor, or R-sensor, provides information on the radial distance from the beam axis. The third coordinate is provided by knowledge of the position of each sensor plane within the experiment. The $r z$ tracking requirement imposes the additional constraint that the VELO circular strips should be centered as perfectly as possible around the beam axis. The result of simulation studies showing how the trigger performance would degrade as a function of various VELO R-sensor misalignments [31] indicate that the R-sensors should be mounted with a mechanical accuracy of better than $20 \mu \mathrm{m}$ in $x$ and $y$ relative to each other within each half, and the two halves should be aligned to better than $100 \mu \mathrm{m}$ relative to each other in these coordinates. The number of strips for both sensor types needed to satisfy the competing requirements of the LHCb environment, physics and a budgetary limit, is about 180000 channels.

\section{Environmental}

The VELO detector will be operated in an extreme radiation environment with strongly nonuniform fluences. The damage to silicon in the most irradiated area for one nominal year of running, i.e. an accumulated luminosity of $2 \mathrm{fb}^{-1}$, is equivalent to that of $1 \mathrm{MeV}$ neutrons with a flux of $1.3 \times 10^{14} \mathrm{n}_{\mathrm{eq}} / \mathrm{cm}^{2}$, whereas the irradiation in the outer regions does not exceed a flux of $5 \times 10^{12} \mathrm{n}_{\mathrm{eq}} / \mathrm{cm}^{2}$. The detector is required to sustain 3 years of nominal LHCb operation. In order to evacuate the heat generated in the sensor electronics (in vacuum) and to minimize radiationinduced effects, the VELO cooling system was required to be capable of maintaining the sensors at a temperature between -10 and $0^{\circ} \mathrm{C}$ with a heat dissipation of about $24 \mathrm{~W}$ per sensor and hybrid. To increase the sensor lifetime, continuous cooling after irradiation was also requested (with the aim to expose the irradiated sensors to room temperature for periods shorter than 1 week per year).

The sensor full depletion voltage is expected to increase with fluence. The ability to increase the operational bias voltage to ensure full depletion during the 3 years lifetime of the sensors was imposed as a further requirement.

\section{Machine integration constraints}

The required performance demands positioning of the sensitive area of the detectors as close as possible to the beams and with a minimum amount of material in the detector acceptance. This is best accomplished by operating the silicon sensors in vacuum. As a consequence, integration into the LHC machine became a central issue in the design of the VELO, imposing a number of special constraints which are briefly discussed here.

- The amount of material in front of the silicon detector is mainly determined by the necessity to shield against RF pickup and the mechanical constraint of building a sufficiently rigid foil. The detectors operate in a secondary vacuum and hence the foils are not required to withstand atmospheric pressure. However, the design of the vacuum system had to ensure that the pressure difference between detector and beam vacuum never be so large as to cause inelastic deformations of the detector box. The VELO surfaces exposed to beam-induced bombardment (secondary electrons, ions, synchrotron radiation) needed to be coated with suitable material in order to maintain beam-induced effects, such as electron multipacting and gas desorption, at acceptable levels for efficient LHC and LHCb operation. The LHC 
beam vacuum chamber, and therefore also the VELO vacuum vessel, were required to be bakeable (to $160^{\circ} \mathrm{C}$ in the case of the VELO).

- A short track extrapolation distance leads to a better impact parameter measurement. Therefore, the innermost radius of the sensors should be as small as possible. In practice, this is limited by the aperture required by the LHC machine. During physics running conditions, the RMS spread of the beams will be less than $100 \mu \mathrm{m}$, but for safety reasons, the closest approach allowed to the nominal beam axis is $5 \mathrm{~mm}$. This value is dominated by the yet unknown closed-orbit variations of the LHC and could be reduced in an upgraded detector. To this must be added the thickness of the RF-foil, the clearance between the RF-foil and the sensors, and the design of about $1 \mathrm{~mm}$ of guard-ring structures on the silicon. Taking everything into account, the sensitive area can only start at a radius of about $8 \mathrm{~mm}$.

- During injection, the aperture required by the LHC machine increases, necessitating retraction of the two detector halves by $3 \mathrm{~cm}$, which brings the movable parts into the shadow of the LHCb beampipe ( $54 \mathrm{~mm}$ diameter). Furthermore, the repeatability of the beam positions could not be guaranteed, initially, to be better than a few $\mathrm{mm}$. This imposed that the VELO detectors be mounted on a remote-controllable positioning system, allowing fine adjustment in the $x$ and $y$ directions.

- The need for shielding against RF pickup from the LHC beams, and the need to protect the LHC vacuum from outgassing of the detector modules, required a protection to be placed around the detector modules. This function is carried out by the RF-foils, which represent a major fraction of the VELO material budget in the LHCb acceptance. In addition, the beam bunches passing through the VELO structures will generate wake fields which can affect the LHC beams. The RF foils, together with wake field suppressors which provide the connection to the rest of the beampipe, also provide the function of suppressing wake fields by providing continuous conductive surfaces which guide the mirror charges from one end of the VELO vessel to the other. These issues have been addressed in detail [32] and are further discussed in section 5.1.3.

\subsubsection{Sensors and modules}

\section{Sensors}

The severe radiation environment at $8 \mathrm{~mm}$ from the LHC beam axis required the adoption of a radiation tolerant technology. The choice was n-implants in n-bulk technology with strip isolation achieved through the use of a p-spray. The minimum pitch achievable ${ }^{4}$ using this technology was approximately $32 \mu \mathrm{m}$, depending on the precise structure of the readout strips. For both the $\mathrm{R}$ and $\phi$-sensors the minimum pitch is designed to be at the inner radius to optimize the vertex resolution.

The conceptual layout of the strips on the sensors is illustrated in figure 5.4. For the R-sensor the diode implants are concentric semi-circles with their centre at the nominal LHC beam position. In order to minimize the occupancy each strip is subdivided into four $45^{\circ}$ regions. This also has the beneficial effect of reducing the strip capacitance. The minimum pitch at the innermost radius

\footnotetext{
${ }^{4}$ The company chosen to fabricate the LHCb sensors was Micron Semiconductor Ltd.
} 


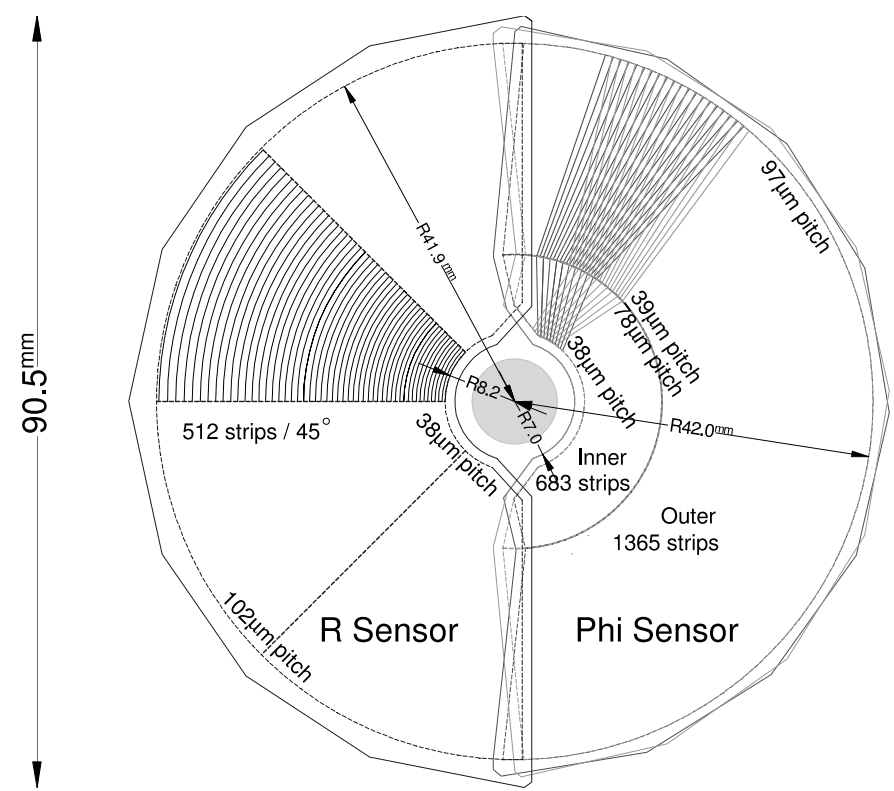

Figure 5.4: Sketch illustrating the $r \phi$ geometry of the VELO sensors. For clarity, only a portion of the strips are illustrated. In the $\phi$-sensor, the strips on two adjacent modules are indicated, to highlight the stereo angle. The different arrangement of the bonding pads leads to the slightly larger radius of the $\mathrm{R}$-sensor; the sensitive area is identical.

is $38 \mu \mathrm{m}$, increasing linearly to $101.6 \mu \mathrm{m}$ at the outer radius of $41.9 \mathrm{~mm}$. This ensures that measurements along the track contribute to the impact parameter precision with roughly equal weight.

The $\phi$-sensor is designed to readout the orthogonal coordinate to the R-sensor. In the simplest possible design these strips would run radially from the inner to the outer radius and point at the nominal LHC beam position with the pitch increasing linearly with radius starting with a pitch of $35.5 \mu \mathrm{m}$. However, this would result in unacceptably high strip occupancies and too large a strip pitch at the outer edge of the sensor. Hence, the $\phi$-sensor is subdivided into two regions, inner and outer. The outer region starts at a radius of $17.25 \mathrm{~mm}$ and its pitch is set to be roughly half $(39.3 \mu \mathrm{m})$ that of the inner region $(78.3 \mu \mathrm{m})$, which ends at the same radius. The design of the strips in the $\phi$-sensor is complicated by the introduction of a skew to improve pattern recognition. At $8 \mathrm{~mm}$ from the beam the inner strips have an angle of approximately $20^{\circ}$ to the radial whereas the outer strips make an angle of approximately $10^{\circ}$ to the radial at $17 \mathrm{~mm}$. The skew of inner and outer sections is reversed giving the strips a distinctive $d o g$-leg design. The modules are placed so that adjacent $\phi$-sensors have the opposite skew with respect to the each other. This ensures that adjacent stations are able to distinguish ghost hits from true hits through the use of a traditional stereo view. The principal characteristics of the VELO sensors are summarized in table 5.1.

The technology utilized in both the R- and $\phi$-sensors is otherwise identical. Both sets of sensors are $300 \mu \mathrm{m}$ thick. Readout of both $\mathrm{R}$ - and $\phi$-sensors is at the outer radius and requires the use of a second layer of metal (a routing layer or double metal) isolated from the AC-coupled diode strips by approximately $3 \mu \mathrm{m}$ of chemically vapour deposited (CVD) $\mathrm{SiO}_{2}$. The second metal layer is connected to the first metal layer by wet etched vias. The strips are biased using 
Table 5.1: Principal characteristics of VELO sensors.

\begin{tabular}{|l|c|c|}
\hline & R sensor & $\phi$-sensor \\
\hline number of sensors & $42+4(\mathrm{VETO})$ & 42 \\
readout channels per sensor & 2048 & 2048 \\
sensor thickness & $300 \mu \mathrm{m}$ & $300 \mu \mathrm{m}$ \\
smallest pitch & $40 \mu \mathrm{m}$ & $38 \mu \mathrm{m}$ \\
largest pitch & $102 \mu \mathrm{m}$ & $97 \mu \mathrm{m}$ \\
length of shortest strip & $3.8 \mathrm{~mm}$ & $5.9 \mathrm{~mm}$ \\
length of longest strip & $33.8 \mathrm{~mm}$ & $24.9 \mathrm{~mm}$ \\
inner radius of active area & $8.2 \mathrm{~mm}$ & $8.2 \mathrm{~mm}$ \\
outer radius of active area & $42 \mathrm{~mm}$ & $42 \mathrm{~mm}$ \\
angular coverage & $182 \mathrm{deg}$ & $\approx 182 \mathrm{deg}$ \\
stereo angle & - & $10-20 \mathrm{deg}$ \\
double metal layer & yes & yes \\
average occupancy & $1.1 \%$ & $1.1 / 0.7 \%$ inner/outer \\
\hline
\end{tabular}

polysilicon $1 \mathrm{M} \Omega$ resistors and both detectors are protected by an implanted guard ring structure.

The pitch as a function of the radius $r$ in $\mu \mathrm{m}$ increases linearly and is given by the following expressions:

$$
\begin{array}{ll}
\mathrm{R} \text { - sensor : } & 40+(101.6-40) \times \frac{r-8190}{41949-8190} \\
\phi-\text { sensor : } & 37.7+(79.5-37.7) \times \frac{r-8170}{17250-8170}(r<17250) \\
\phi-\text { sensor : } & 39.8+(96.9-39.8) \times \frac{r-17250}{42000-17250} \quad(r>17250)
\end{array}
$$

The sensors were developed for high radiation tolerance. Early prototype detectors used pstop isolation. This was later replaced by $\mathrm{p}$-spray isolated detectors which showed much higher resistance to micro-discharges. The $\mathrm{n}^{+} \mathrm{n}$ design was compared with an almost geometrically identical $\mathrm{p}^{+} \mathrm{n}$ design and was shown to have much better radiation characteristics as measured by charge collection as a function of voltage.

Prototype sensors were also irradiated with non-uniform fluence in order to study the effects of cluster bias due to inhomogeneous irradiation. It was shown that the transverse electric fields produce less than $2 \mu \mathrm{m}$ effects on the cluster centroid.

A subset of the production sensors were exposed to a high neutron fluence $(1.3 \times$ $10^{14} \mathrm{n}_{\mathrm{eq}} / \mathrm{cm}^{2}$ ) representing 1 year of operation at nominal luminosity. A strong suppression of surface breakdown effects was demonstrated. The evolution of the depletion voltage was found to correspond to the expectation over LHC operation: with an integrated luminosity of $2 \mathrm{fb}^{-1}$ per year, the maximum deliverable full depletion voltage $(500 \mathrm{~V})$ is reached after approximately 3 years. During production the possibility arose of manufacturing full size $\mathrm{n}^{+} \mathrm{p}$ sensors. These are expected to have similar long term radiation resistance characteristics to the $n^{+} n$ technology, but feature some advantages, principally in cost of manufacture due to the fact that double sided processing is not needed. One full size module was produced in this technology and installed in one of the most upstream slots. It is forseen to replace all the VELO modules after damage due to accumulated radiation or beam accidents. The replacement modules will be constructed in the $\mathrm{n}^{+} \mathrm{p}$ technology [33]. 


\section{Modules}

The module has three basic functions. Firstly it must hold the sensors in a fixed position relative to the module support. Secondly it provides and connects the electrical readout to the sensors. Finally it must enable thermal management of the modules which are operating in vacuum.

Each module is designed to hold the sensors in place to better than $50 \mu \mathrm{m}$ in the plane perpendicular to the beam and within $800 \mu \mathrm{m}$ along the direction of the beam. Sensor-to-sensor alignment (within a module) is designed to be better than $20 \mu \mathrm{m}$.

The module is comprised of a substrate, for thermal management and stability, onto which two circuits are laminated. This forms the hybrid. The substrate is fabricated in-house and is approximately $120 \times 170 \times 1 \mathrm{~mm}$. It has a core of $400 \mu \mathrm{m}$ thick thermal pyrolytic graphite (TPG), and is encapsulated, on each side, with $250 \mu \mathrm{m}$ of carbon fibre (CF). A CF frame of about $7 \mathrm{~mm}$ thickness surrounds the TPG and is bonded directly to the CF encapsulation to prevent delamination. The TPG is designed to carry a maximum load of $32 \mathrm{~W}$ away from the front-end chips. A semicircular hole is cut into the substrate under the region where the detectors are glued. Particular attention in the design and fabrication process is given to producing almost planar hybrids, to simplify the subsequent module production. Typical non-planarities of order $250 \mu$ m were achieved.

The circuits ${ }^{5}$ were commercially hand populated to minimize exposure of the hybrid to high temperature and hence the possibility of delamination. The sensor front-end ASICs (Beetle 1.5 [34]) were then glued to the circuits. A total of 32 Beetle chips are used in each module. Kapton pitch adaptors, ${ }^{6}$ were glued to the circuits in order to facilitate the wire bonding of the sensors to the Beetle chips. Sensors were glued to the double-sided hybrid with a sensor-to-sensor accuracy of better than $10 \mu \mathrm{m}$. The sensors were bonded to the electronics using a combination of H\&K 710 and K\&S 8090 bonding machines with $25 \mu \mathrm{m}$ thick Al wire. After bonding and final testing $99.4 \%$ of all strips were operational, with no sensor having more than 30/2048 faulty channels.

The final mechanical mounting of the hybrid was to the module pedestal and base. The pedestal is a low mass CF fibre construction designed to hold the hybrid stably. It is a hollow rigid structure approximately $140 \times 150 \times 10 \mathrm{~mm}$. Holes are drilled where appropriate to reduce the possibility of outgassing. One end was glued to the hybrid and the other to a CF base which contains two precisely manufactured invar feet. The design of the base allows repeatable mounting of the module to the module support with a precision a better than $10 \mu \mathrm{m}$. The accuracy of the final assembled modules satisfied the design criteria. The complete module, in schematic and after final assembly, is illustrated in figure 5.5.

The thermal performance of the modules was required to be such that the silicon could be operated below zero degrees, for a minimum cooling liquid temperature of $-30^{\circ} \mathrm{C}$. The performance of each module was monitored individually in a vacuum tank during construction with a thermal camera, as illustrated in figure 5.6. After the final cooling connections had been made on the module bases, all modules were rechecked for their thermal performance in vacuum. The temperature difference between the cooling blocks and the silicon is estimated to be about $(21.4 \pm 1)^{\circ} \mathrm{C}$ for individually cooled modules, and an improvement of up to $2^{\circ} \mathrm{C}$ is expected when the modules are

\footnotetext{
${ }^{5}$ The circuits were fabricated and laminated to the substrates by Stevenage Circuits, Ltd.

${ }^{6}$ The pitch adapters were manufactured at CERN.
} 

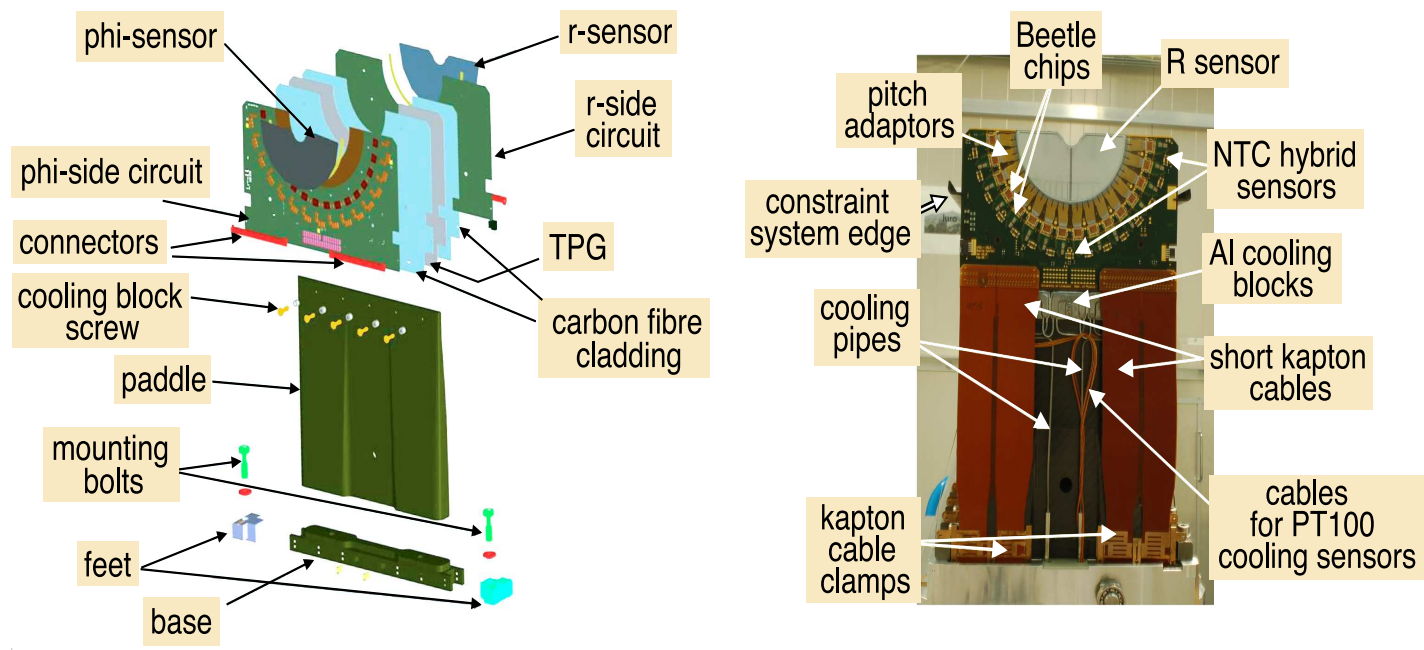

Figure 5.5: The left part of the figure illustrates the principal components of the VELO module. The right part of the figure is a photograph of the module as finally mounted. The short kapton cables are visible, along with the clamps at the base which prevent any long kapton cable movement coupling to the module itself. The aluminium blocks encasing the stainless steel $\mathrm{CO}_{2}$ cooling tubes are also partially visible.
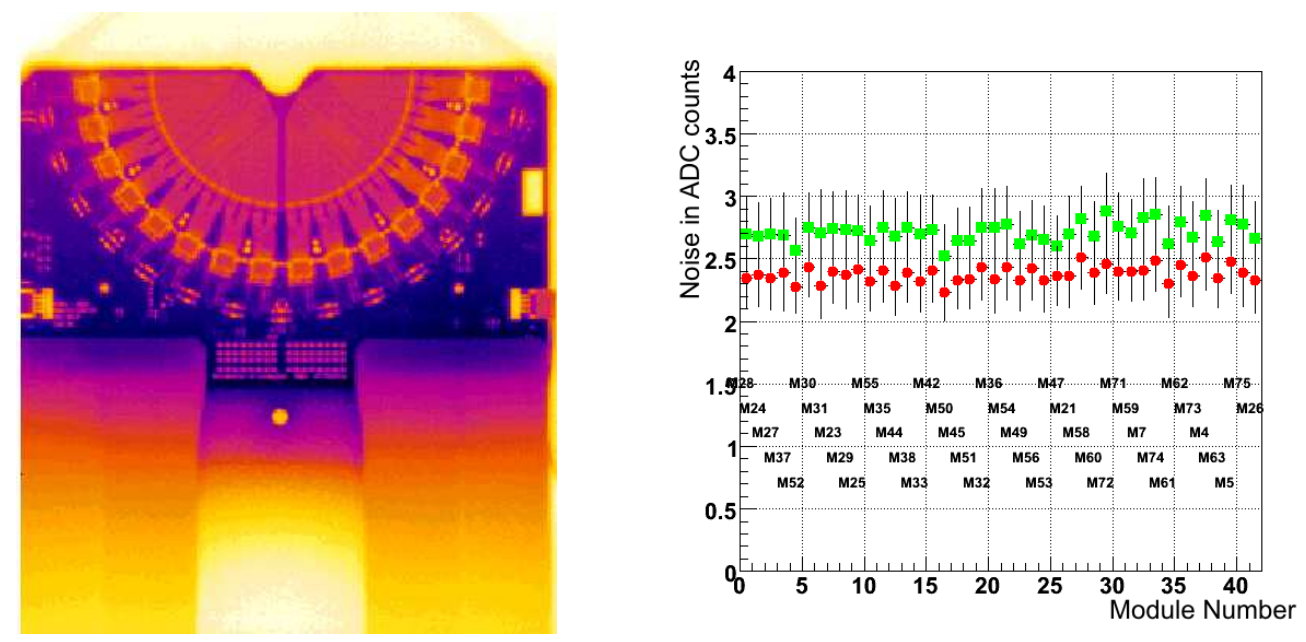

Figure 5.6: Quality control of the VELO modules. The left part of the figure shows an example of a thermograph which was routinely taken on both sides of the module during the construction. The right plot shows an example of a noise uniformity measurement for all the $\phi$-sensors. The green squares are before common mode correction, and the red circles after the common mode correction. The error bars show the noise spread which is due to varying strip capacitances on the sensor.

cooled simultaneously. At the same time, the noise uniformity of the modules was checked, as shown in figure 5.6.

Each module was transported to CERN in a dedicated transport box [35] and submitted to a visual inspection with a high resolution microscope, including the survey of all bond wires, on 


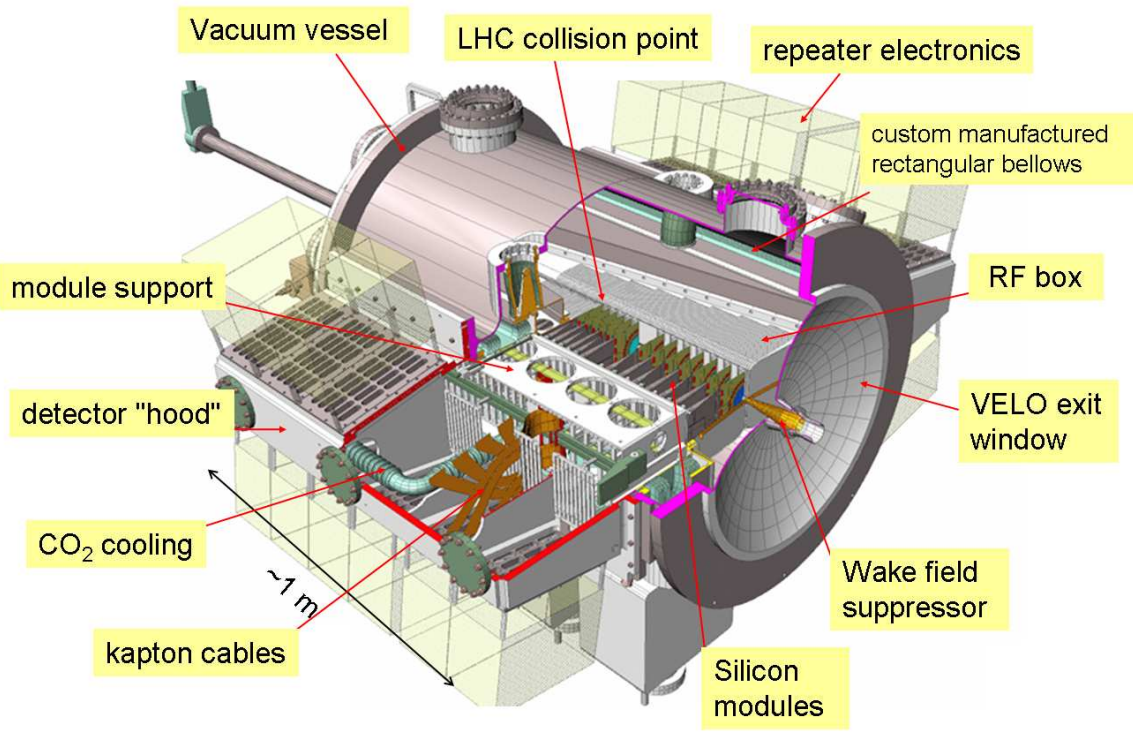

Figure 5.7: Overview of the VELO vacuum vessel.

reception. After this it underwent a 16 hour burn-in [36] consisting of a sequence of powering, cooling and data taking cycles, in vacuum. This was designed to uncover any inherent weaknesses introduced to the modules during manufacturing. Of all the modules transported, one was finally taken as a reserve as a result of the burn-in procedure, and a second was rejected after assembly due to a slightly worse thermal performance.

\subsubsection{Mechanics}

\section{Introduction}

The ultra high vacuum requirements of the LHC ring, the necessity for wake field suppression, the need to shield the detectors from electromagnetic effects induced by the high frequency beam structure, and the necessity to retract the detectors by $30 \mathrm{~mm}$ from the interaction region during injection of a new LHC fill, make the VELO mechanical design demanding. To meet all these constraints, a design with two detector halves was chosen, each placed inside a thin-walled aluminum box, as introduced in the previous sections. Aluminum was chosen since it has a relatively low $\mathrm{Z}$ (resulting in a small radiation length), good electrical conductivity, and can be machined quite easily. The side walls of these boxes are $0.5 \mathrm{~mm}$ thick. In order to allow for overlap in the two detector halves, the top surfaces of these vacuum boxes have a corrugated shape and are made from $0.3 \mathrm{~mm}$ thick AlMg3 foil (an aluminum alloy with 3\% magnesium). The two detector boxes are placed in a $1.4 \mathrm{~m}$ long vacuum vessel with a diameter of $1.1 \mathrm{~m}$. The whole assembly is shown in figure 5.7. Two rectangular bellows allow for the movement of the detector boxes inside the vacuum system. Each detector support is connected via three spheres on holders placed within circular bellows to 


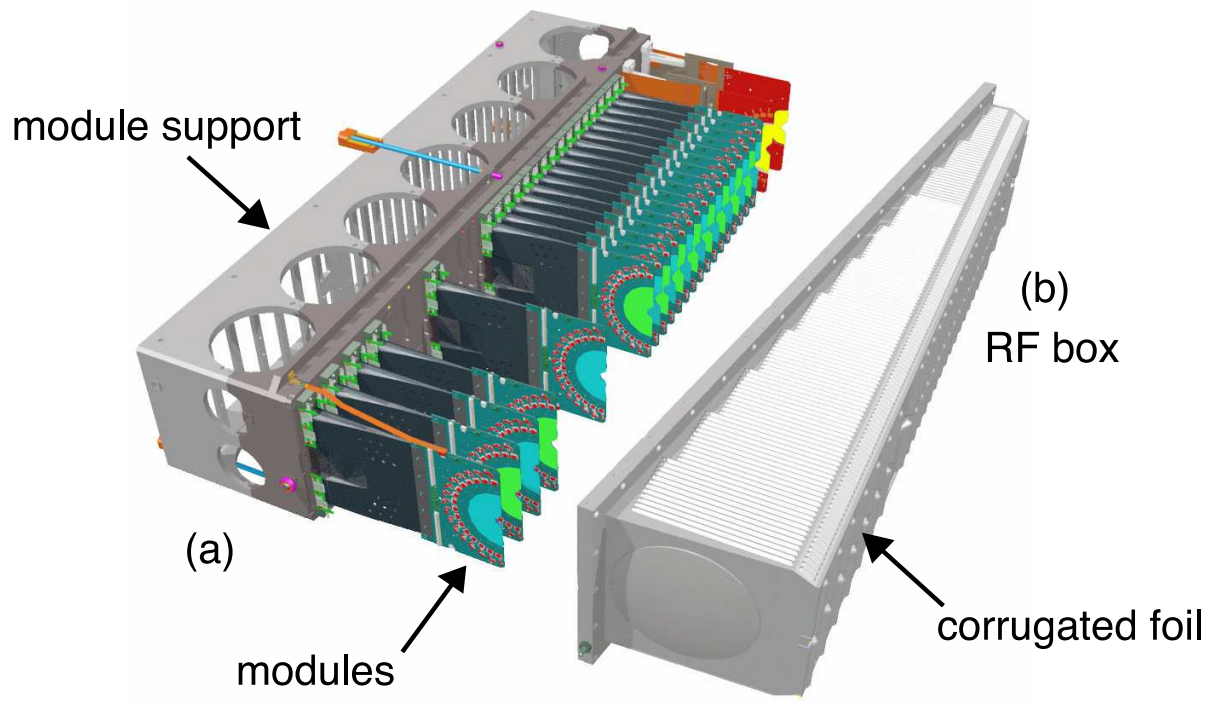

Figure 5.8: Exploded view of the module support and the modules (a), and the RF box (b). The corrugated foil on the front face of the box, which forms a beam passage can be seen. Its form allows the two halves to overlap when in the closed position.

the movement mechanism that is located outside the vacuum vessel. The module support, illustrated in figure 5.8, is mounted on bearings which lie in precisely machined slots inside the detector support and is bolted into position against three precision surfaces. The positioning of the modules on the module support is fully constrained with a combination of a slot and a dowel pin. The positioning of the modules relative to one another is determined by the precision of the machining of the module support. The positioning of the two halves relative to each other is determined by the precision of the module support, together with its positioning inside the detector support, which is adjustable. To suppress wake field effects, the dimension of the beampipe as seen by the proton beams has to vary very gradually. To match the beampipe upstream and downstream from the VELO, wake field suppressors made of $50 \mu \mathrm{m}$ thick copper-beryllium have been constructed, so a good electrical match is provided in both the open and closed positions.

The durability of the wake field suppressors has been tested by performing an opening and closing movement 30,000 times, after which no damage was observed. A photograph of the downstream wake field suppressors is shown in figure 5.9. The exit foil of the vessel consists of a $2 \mathrm{~mm}$ thick aluminum window (see section 3 ).

\section{Movement system}

Before the LHC ring is filled, the detectors have to move away from the interaction region by $30 \mathrm{~mm}$ in order to allow for beam excursions during injection and ramping. After stable beam conditions have been obtained, the detectors should be placed into an optimized position centered in $x$ and $y$ around the interaction region. This position is not exactly known beforehand; it may vary over $\pm 5 \mathrm{~mm}$ in both $x$ and $y$, even from fill to fill. Therefore, a procedure has been developed to determine the beam position with the detectors not completely moved in, and then move to 


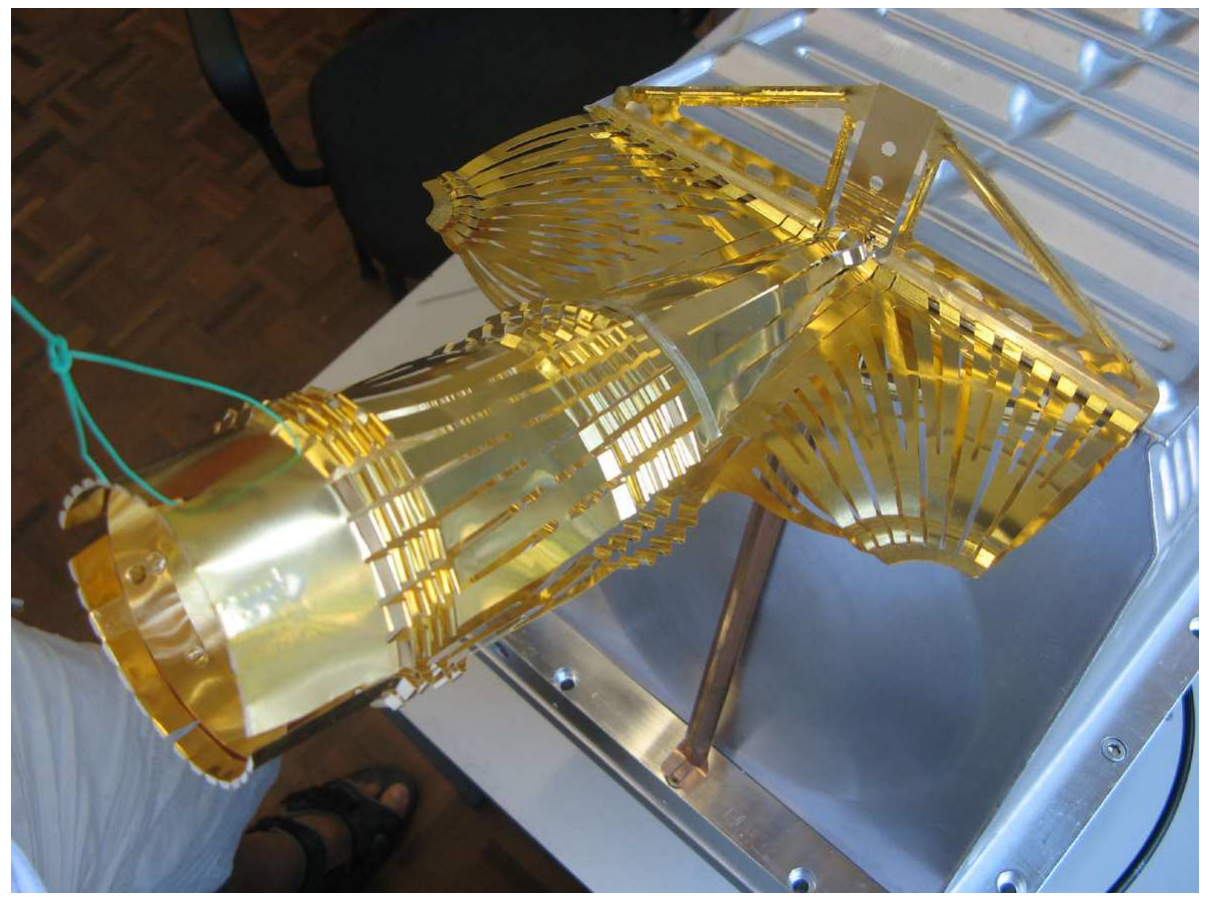

Figure 5.9: Photograph of the downstream wakefield suppressor.

the optimal position. This is performed with a motion mechanism that can bring the detectors to their position with an accuracy of the order of $10 \mu \mathrm{m}$ by means of a stepping motor with resolver readout. Additional potentiometers have been used to verify independently the proper functioning. The motion procedures are controlled by a Programmable Logic Controller (PLC). The boxes can move together in $y$ and independently in $x$, and a series of stops prevents mechanical interference.

\section{Vacuum system}

In the LHC ring, Ultra High Vacuum conditions of better than $10^{-8}$ mbar are required, compatible with reduced beam-induced effects. To maintain these conditions, the beampipes are equipped with a Non-Evaporable Getter (NEG) layer. To maintain the low desorption yield of the NEG coating, the vacuum system will be vented during maintenance with ultra-pure neon. The thin walled detector boxes are expected to plastically deform at a pressure difference of $20 \mathrm{mbar}$ (and to rupture above 50 mbar). Therefore, the detectors also have to be operated under vacuum. Due to outgassing of detectors, hybrids, cables and connectors, a vacuum around $10^{-4}$ mbar is expected. Hence a good separation between the beam and detector vacuum is necessary as exposure to the detector vacuum would saturate and contaminate the NEG material on the inside of the beampipe.

A procedure has been implemented to make sure that during venting and evacuation of the VELO vacuum system the pressure difference between beam and detector vacuum will never exceed 5 mbar overpressure in the detector vacuum and 2 mbar overpressure in the beam vacuum. This asymmetry is designed to maximise the protection of the modules. This is achieved by using dedicated valves and restrictions, that are activated by membrane switches that react at the desired pressure difference. The complete vacuum system between the two sector valves around $\mathrm{LHCb}$ is controlled by a PLC. 


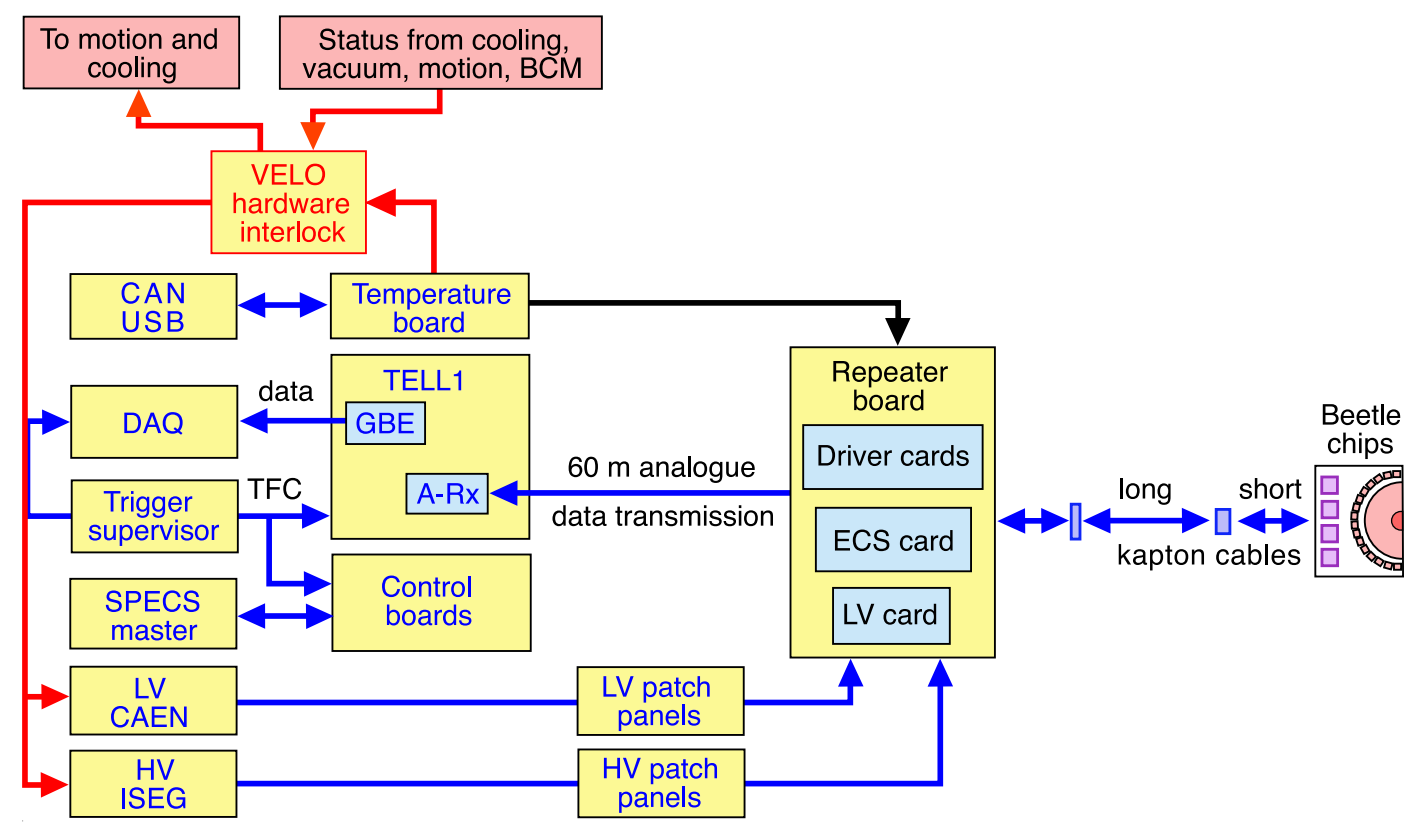

Figure 5.10: VELO readout chain for one side of one module.

\section{Cooling system}

Since the detectors and read-out electronics are operated inside the vacuum system, active cooling is required. Furthermore, in order to limit the effects of radiation damage of the silicon sensors, the irradiated sensors should be operated and kept at temperatures below $-5^{\circ} \mathrm{C}$ at all times. The radiation hard refrigerant in the system is two-phase $\mathrm{CO}_{2}$ cooled by a conventional freon cooler. The liquid $\mathrm{CO}_{2}$ is transported via a $60 \mathrm{~m}$ long transfer line to the VELO, where it is distributed over 27 capillaries per detector half. Each capillary is thermally connected to five cooling blocks that are attached to each detector module. A redundant pumping system is incorporated, so that also during maintenance periods cooling capacity is available. The complete cooling system is controlled by a PLC.

\subsubsection{Electronics chain}

The electronics layout is summarised in figure 5.10, which shows the readout chain for one side of one module. The individual components are discussed in this section.

\section{Beetle chip}

The VELO uses the Beetle, a custom designed radiation hard ASIC based on $0.12 \mu \mathrm{m}$ CMOS technology ${ }^{7}$ with an analog front-end, as the FE chip [34]. The chip was designed with a peaking time and sampling frequency to match the LHC bunch crossing rate of $40 \mathrm{MHz}$, and to be able to readout at a speed which can match the L0 accept rate of $1 \mathrm{MHz}$. For a full discussion of the design requirements see $[28,37]$. Each of the 128 channels consists of a charge sensitive

\footnotetext{
${ }^{7}$ IBM, USA.
} 


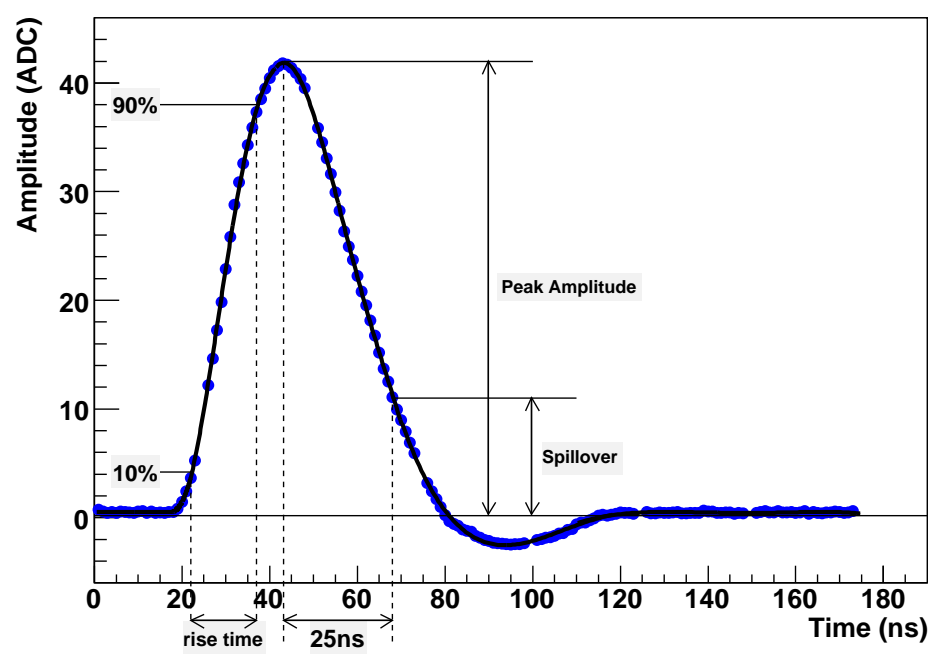

Figure 5.11: Beetle pulse shape as measured for a VELO sensor using test-pulses.

preamplifier/shaper and an analog pipeline of 160 stages designed to match the $\mathrm{L} 04 \mu$ s latency. The data are brought off chip at a clock frequency of $40 \mathrm{MHz}$, with 32 channels multiplexed on each of 4 output lines. This allows the readout of one event to be achieved in $900 \mathrm{~ns}$. The chip specifications required a survival more than 5 years of nominal operation at a dose of $2 \mathrm{MRad}$ per year. In fact the chip is radiation hard against total dose effects of more than 100 MRad. Robustness against single event upset is achieved with redundant logic. It is programmable via a standard I2C interface, which controls the bias settings and various other parameters. The circuit can be tested via a charge injector (test-pulse circuit) with adjustable pulse height. In the case of the pile-up readout, where the data are used in the L0 trigger, a fast binary signal is required. This is achieved by routing the output of the front-end amplifier to a comparator stage with extra output pads on the chip.

For the VELO, the spillover value, or the remnant signal remaining after $25 \mathrm{~ns}$, is of particular importance, and must be below 0.3 of its peak value for reliable HLT performance. Figure 5.11 shows the measured pulse shape on a Beetle fully mounted on a VELO module and bonded to the sensor, using a test-pulse corresponding to a minimum ionising particle in $300 \mu \mathrm{m}$ of silicon, for the bias settings expected for $\mathrm{LHCb}$ operation. The rise time is measured to be $14.7 \pm 0.5 \mathrm{~ns}$ and the spillover $(26.0 \pm 0.6) \%$. For more details on the measurement see [38].

\section{Kapton cables}

During injection of the proton beams in the ring, the detectors have to be retracted by $30 \mathrm{~mm}$. The total number of data and control signals that run between the hybrids and the feed-through flanges at the vessel exceeds 18000 . Kapton cables were chosen as they are thin, flexible and radiation hard. The central part of the cable consists of a $17 \mu \mathrm{m}$ thick copper layer with $150 \mu \mathrm{m}$ wide strips. This layer is covered on each side with a $100 \mu \mathrm{m}$ thick kapton foil, a $17 \mu \mathrm{m}$ thick rolled annealed (RA) copper foil which is used to supply power to the Beetle chips, and a $25 \mu \mathrm{m}$ thick cover kapton foil.

Each cable consists of two parts: a short tail from the hybrid to a fixed connector, and a long cable from this connector to the vacuum feed through on the vessel. 


\section{Repeater boards}

The repeater board (RPT) is located directly outside of the VELO tank inside repeater crates. The RPT function is mainly a repeater for the differential signals arriving at the board, including data signals, Time and Fast Control (TFC, see section 8.3) and FE chips configuration signals. Also, monitoring signals are sent out via the board to the detector slow control system. The RPT carries the voltage regulators required by the FE and the L0 electronics service system. For flexibility in design and mainly for maintenance, the RPT is built as a motherboard hosting several mezzanine cards:

- Four Driver Cards: four driver cards are mounted in the RPT board as mezzanine cards. Each card contains 16 fully differential analog drivers. Because the data streams are sent to the digitizer card via a $60 \mathrm{~m}$ individual shielded twisted-pair cable, the drivers include a line equalizer to compensate distortions introduced by the cable.

- One LV card: the low voltage card provides the power for the FE hybrid, the analog driver cards and the ECS card (c.f. 8.4). Eight radiation hard voltage regulators are mounted on the board. Each voltage is monitored through an amplifier. The card is supplied by three power supplies located at $60 \mathrm{~m}$ cable distance. Sense-lines are used to compensate the voltage drop through the long supply cable.

- One ECS card: the ECS repeats the signals for the I2C configuration bus and controls and monitors the LV regulators. The applied voltage and the current limit signals are multiplexed and sent on differential lines. The ECS card communicates with the control boards located in intermediate crates $15 \mathrm{~m}$ away from the VELO.

Another part implemented on the board is the TFC functionality. These fast LVDS signals are signals required by the FE chips and are sent through a LVDS repeater mounted on the board. The boards and their components underwent radiation tests to confirm that they could tolerate more than ten times the expected level of $73 \mathrm{kRad}$ during 10 years of operation [39].

\section{Control board}

The control board [40] is the heart of the control system of the L0 electronics. The timing signals and fast commands from the Readout Supervisor are distributed to the L0 electronics via the control board. Moreover the configuration data is received by the SPECS slave [41] on the control board and distributed to the configurable components via I2C. The monitored voltages are digitized on the control board and the values are propagated to the ECS system via the SPECS bus. Each control board supports six VELO electronics hybrids or two PU electronics hybrids and four PU optical boards.

\section{Temperature board}

The temperatures of the electronics hybrids and voltage regulators are monitored by the temperature boards. The temperature boards also generate signals to the VELO interlock board, which is the last safety mechanism in case of failure (see below). Each temperature board hosts one ELMB (Embedded Local Monitoring Board [9]) and four Interlock Boxes (IBox [42]), which together 
serve 16 repeater boards. The ELMB has an embedded Atmel ATMega128 processor and communicates with the supervisory system via a CAN bus. It has digital I/O ports, 64 16-bit ADC channels and a voltage reference circuit. This provides a stand-alone system for temperature monitoring read out via a CAN bus. The IBox supplies a voltage across the NTC resistor and a precision resistor in series. The voltage drop across the NTC resistor is compared to a reference on the IBox and two bits per temperature channel are generated signalling the states $O K$, too warm, too cold or error. The ELMB is in this configuration connected in parallel with the IBox, passively monitoring the voltage drop and hence the temperature. The interlock states of the 64 temperature sensors are read out by one temperature board and combined into a common interlock state of the board. This interlock matrix is implemented in an FPGA and provides the facility to monitor the interlock status and mask faulty channels.

\section{VELO specific TELL1 features}

The TELL1 boards of LHCb are described in section 8.2. Specific to the VELO is the digitization of the data on the TELL1 and the complex pre-processing of the data.

Due to the high radiation levels and space constraints, the digitization of the data and the use of optical drivers close to the detector were discarded as solutions for the VELO. As a consequence, the analog data are directly transmitted to the TELL1 board and digitized on plug-in cards. Each TELL1 board deals with the data from one sensor, i.e. 64 analog links, and features 4 A-Rx cards. One A-Rx card provides 16 channels of 10-bit ADC's to sample the analog data from 4 Beetle chips at $40 \mathrm{MHz}$. In order to compensate for the time skew of the signals resulting from different cable lengths the sampling time can be chosen by phase adjustable programmable clocks, individually for each ADC channel.

After digitization, the TELL1 performs data processing in the ppFPGAs (pre-processing FPGAs, of which there are four per TELL1) before sending the zero-suppressed data to the trigger farm [43]. The first stages are implemented in 10-bits and the precision is then reduced to 8 bits. The steps in this data processing include pedestal subtraction, cross-talk removal, channel re-ordering, common mode suppression, and clustering. Pedestal subtraction is implemented with a running average pedestal following algorithm available, if required, to calculate the value for each channel. Although most of the signal distortion in the long data cable is removed already through frequency compensation, a Finite Impulse Response Filter is used to correct for cross-talk and further improve the system performance. In both the R- and the $\phi$-sensors, adjacent physical strips are scrambled in the readout chain. For the clustering it is essential to bring them back into order by implementing a channel reordering step. Common mode suppression algorithms may be performed in both the readout chip channel ordering and in the physical strip ordering. This allows correlated noise pickup or other baseline level shifts on the sensors or in the front-end chips and readout chain to be corrected. A mean common mode algorithm that corrects for a constant shift and a linear common mode algorithm that corrects for both a constant and a slope are implemented over appropriate groupings of channels. The last processing step is the clustering [44]. Strips are selected as seeding strips if the signal passes a certain seeding threshold. Strips next to the seeding strips are included in the cluster if their signals are above the inclusion threshold cut. A cluster can be formed by a maximum of four strips. The cluster centre is calculated with a 3-bit precision. 
The VELO raw data are sent in a format that allows a fast access of the cluster information by the trigger by sending the calculated 14-bit cluster position. For more refined calculations of the cluster centre and offline analyses, the ADC information of all strips is added in another data block [45].

\section{The VELO hardware interlock system}

The VELO is protected with a simple and failsafe hardware interlock system. An overview of its functionality is given in [46]. Switching on the low voltage when the VELO is not properly cooled is for instance prevented by this system. The status of the cooling, vacuum, motion and detector front-end systems and the BCM (cf. section 3) are combined in an interlock logic unit and fed back to high and low voltage systems and to the motion and cooling system. The interlock unit is based on a FPGA to provide flexibility and possible future interfacing to PVSS (see section 8.4) for monitoring. All input signals are continuously monitored and their status shown on the front panel LED's. The inputs are fail safe such that any disconnection or power loss will result in a bad status and the interlocks will fire. Any of the inputs may additionally be forced to a good status by internal switches for debugging or override purposes, and this is also indicated on the front panel. The interlock outputs are also fail safe such that cable removal or power failure will result in module power and cooling being removed. The status of the outputs is shown on the front panel and all individual outputs can be overidden by internal switches. Hardware signals are also exchanged between motion, cooling and vacuum PLCs to prevent, for example, cooling when there is no vacuum. As the VELO cannot be allowed to move in while the LHC beam is not stable, beam inhibit and beam status signals are exchanged with the BCM and the LHC beam interlock system [20]. Direct signals from the LHC sector valves and the neon injection system are given to the VELO vacuum system to prevent venting, evacuating or neon injection when one of these systems is not ready.

\section{LV system}

The VELO and pile-up low voltage system is based on a multi-channel power supply system. ${ }^{8}$ All the power supplies are installed in the detector counting house. Each module has 12 fully floating channels and can supply 4 repeater boards. Each channel has its own voltage sense-line to permit a correction to be made for any voltage drop over the long distance cable. Each repeater board requires three voltages which are supplied over shielded twisted-pairs. The LV power supplies interface to the hardware interlock system.

\section{HV system}

The silicon sensors will be operated under a reverse bias ranging from 100-500 V, with the operating voltage being increased as the sensors undergo radiation damage. The biasing scheme ties the $n^{+}$strips to ground, and applies a negative voltage to the backplane. The high voltage system utilises 6 power supply modules, ${ }^{9}$ each controlling up to 16 sensors, which are housed in an

\footnotetext{
${ }^{8}$ Manufactured by CAEN.

${ }^{9}$ Manufactured by ISEG Spezialelektronik GmbH.
} 
uninterruptible power supply crate in the detector counting house. The output is fed via 37-core cables to a patch panel in the counting house. Long 56-core cables connect the counting house to a second patch panel located near the detector. Three-core cables then provide the high voltage, high voltage guard and ground to the repeater board of each module. The high voltage guard connection provides the voltage to a guard trace on the sensor hybrids, which surrounds the detector high voltage trace, thus reducing possibilities of shorting. The high voltage guard line can be connected to the high voltage or left floating by adjustment of jumper switches in the counting house patch panel. The high voltage system is controlled through PVSS. Voltage and current limits are also set in hardware on the power supply units. The high voltage power supplies interface to the hardware interlock system.

\section{Grounding and power supply}

The power distribution and grounding scheme partitioning of the VELO and the pile-up electronics follows the detector topology. Each silicon detector with its hybrid forms a group. The number of groups connected to the same power distribution connection is $\mathrm{k}$ ept to a minimum. There are in total 84 VELO and 4 pile-up hybrids with $16 \mathrm{FE}$ chips each installed inside the tank. The tank is the central part of the system and is connected to the LHC machine. The LHC machine earth cables form part of the cavern network grounding. All metallic devices, such as electrical cabinets and cable trays, have ground connection to the main VELO tank support. All electrical devices forming part of the cooling and vacuum system must be grounded for safety reasons. All components connected to the main power network line are equipped with protection circuits and electrically floating devices made of conductive material are not permitted.

The analog FE electronics is potentially prone to pickup from external noise sources. The effects are minimised by keeping the signal current path as short as practicable. The reference voltage of the charge preamplifier in the FE chip is internally connected to the ground pins of the chip. The current loop for the signal generated in the sensor is closed through the sensor bias line. The bias line is AC-connected to the reference ground of the FE chip. The silicon detectors and hybrids are located close to the detector RF shield, which is connected to the ground. Potential differences between these two components give rise to the main source of noise in the detector. This is minimised by keeping the hybrid ground plane at the same potential as the RF shield. Each hybrid ground plane is tied to the module support with a silver-plated copper grounding strap.

\subsubsection{Material budget}

The material budget was investigated in the simulation by generating straight tracks originating from the interaction point and extrapolating them through the VELO. The radiation length seen by the track was calculated for each volume it crossed in the geometry description. The average radiation length is shown as a function of the azimuthal angle $\phi$ and the pseudorapidity $\eta$, with $1.6<\eta<4.9$ in figure 5.12. The contribution of the major components is also shown. The average radiation length of the VELO is $17.5 \%$ of a radiation length with the largest contribution coming from the RF-foil. 

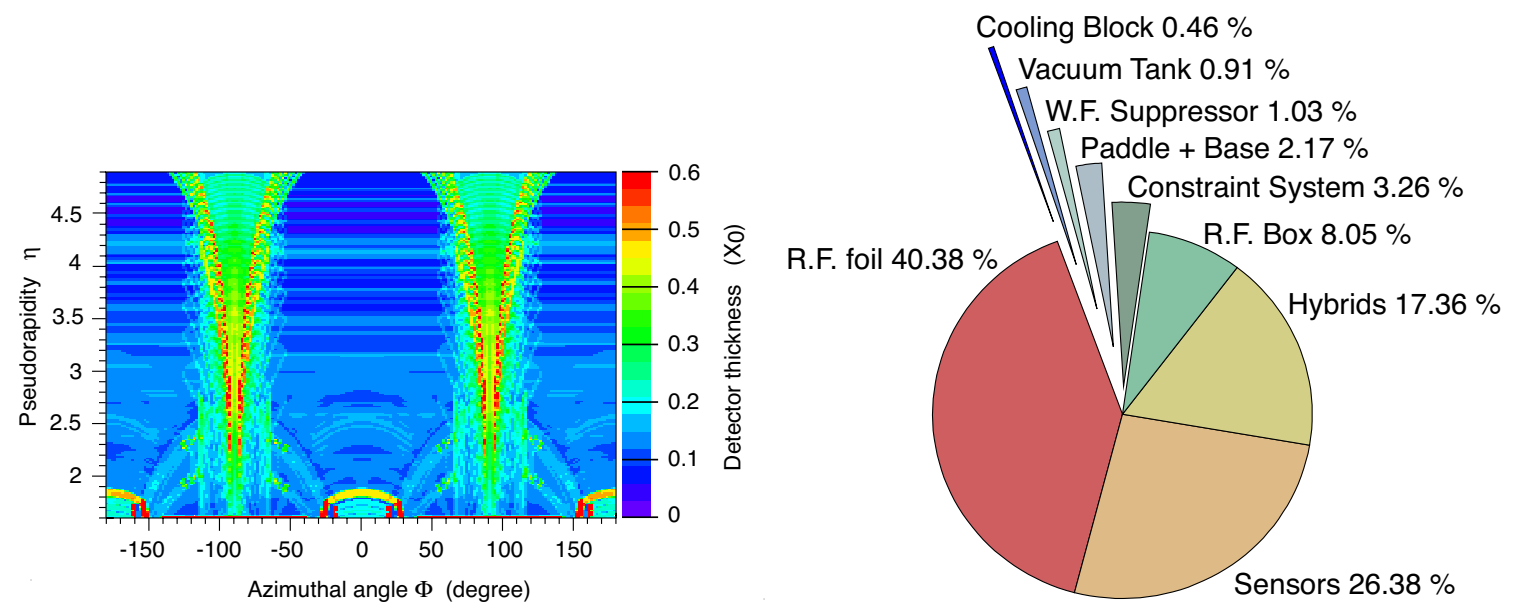

Figure 5.12: The average radiation length seen by particles passing through the VELO as a function of azimuthal angle, $\phi$, and pseudorapidity, $\eta$, with $1.6<\eta<4$.9. For a uniform sampling over these coordinates the average contribution to the radiation length is $0.175 \mathrm{X}_{0}$.

\subsubsection{Test Beam detector commissioning}

Test Beam studies of two different configurations of fully instrumented VELO modules were carried out at the CERN H8 experimental area, using $180 \mathrm{GeV} \pi$ beams with tuneable intensity and spot size. An external trigger was provided by a scintillator telescope that could be configured to select events including single tracks crossing the detectors without interactions, multiple track events produced by interaction in the detectors or on the entrance window of their enclosure, and, in the second data taking cycle, interactions in target modules installed in the module array. A total of 4 target planes, mounted in two modules were used. Each plane included a small Cu disk, with a thickness of $300 \mu \mathrm{m}$ and a $2 \mathrm{~mm}$ diameter, centered on the beam axis, as well as $5 \mathrm{~mm}$ diameter $\mathrm{Cu}$ disks with a radial displacement of $15 \mathrm{~mm}$ from the beam axis. The latter targets had the purpose of investigating the vertex reconstruction capabilities of the detector in the retracted position (open VELO).

The first configuration included 3 fully instrumented half-disk pre-production modules built using $200 \mu \mathrm{m}$ sensors, enclosed in a box providing accurate positioning on a table including a rotating stage that allowed data taking with the detectors oriented at an angle with respect to the beam direction. Thus the detectors were operated in air, at a typical temperature of $40-50^{\circ} \mathrm{C}$. Only $1 / 4$ of the electronics was read out, due to limitations in the available hardware. This data set provided considerable insight into the performance of the final detector modules. Figure 5.13 shows that the measured cluster multiplicity as a function of the strip number is in good agreement with the expectations based on a dedicated MonteCarlo simulation [47]. Based on Monte-Carlo simulation and the performance of the electronics a resolution of about $6 \mu \mathrm{m}$ can be achieved at the inner radius of the sensors. This optimal resolution will be obtained by utilizing a non-linear charge-sharing algorithm that depends on the incident angle of the tracks (for the R-sensor) and the irradiation level of the sensors. All the parameters affecting the performance of the sensors and the readout electronics were explored, for example data was taken with the sensors biased with different high 

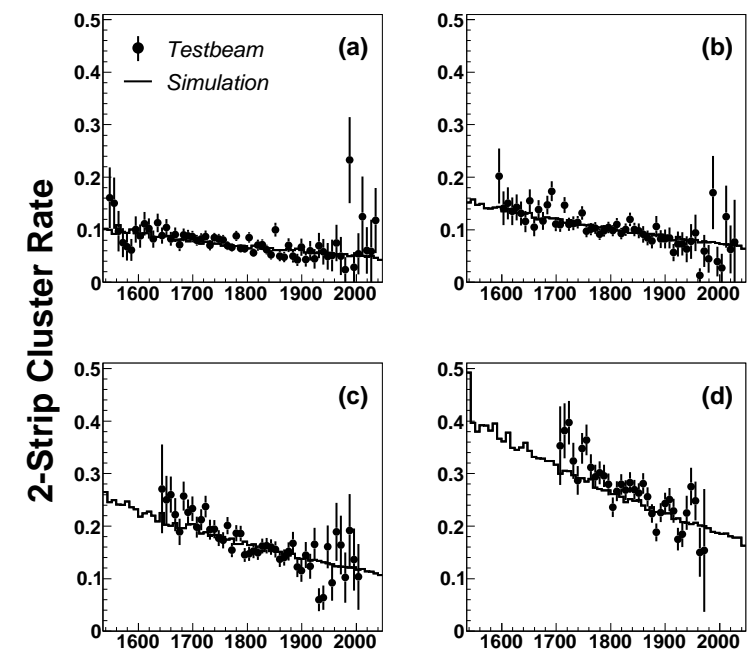

Software Strip ID

Figure 5.13: Fraction of two strip clusters in R-sensors, for different beam track angles with respect to the normal to the detector planes: a) $0^{\circ}$, b) $5^{\circ}$, c) $10^{\circ}$ d) $15^{\circ}$. The horizontal axis represents the strip number, corresponding to a progressively coarser inter-strip pitch.

voltages, and the operating parameters of the Beetle chip were changed. In addition, the noise performance was studied both with random triggers, and with electronic calibration runs. These data validate in a multi-detector configuration the laboratory characterization of the individual module components. The data acquisition and monitoring infrastructure planned for the experiment were used. The system performance was excellent.

The second data set was taken using a system of 10 production modules including $300 \mu \mathrm{m}$ thick sensors, mounted in the vacuum tank built for the final system, reading out 6 of them in different combinations, depending upon the trigger scheme chosen. Most of the data was taken in vacuum (below $10^{-3} \mathrm{mbar}$ ) and at a temperature of about $-3^{\circ} \mathrm{C}$. This test beam cycle provided valuable operating experience with the cooling system built for the experiment and with vacuum implementation and monitoring. Data were taken in air at room temperature, and it was confirmed that the module positions remained stable through the transition between air and vacuum and throughout temperature cycling.

The production detectors operated with a signal to noise ratio of between 17 and 25 , depending on strip length, where signal is defined as the most probable value of the charge cluster produced by a minimum ionizing particle (MIP) and noise is the incoherent noise measured from calibration data after subtracting the coherent noise component. Their performance proved to be remarkably stable throughout the 14 days of data taking. Single track and interaction trigger data complement the first data set in assessing the hit resolution as a function of angle. In addition, target data produced reconstructed primary vertices from all the targets that help in tuning the vertex reconstruction algorithms. 


\subsubsection{VELO software}

\section{Reconstruction software}

The TELL1 data processing boards (see sections 5.1.4 and 8.2) perform a set of processing algorithms on the raw VELO data and identify clusters for use in the trigger and for off-line physics analysis. For use in the trigger the clusters are stored in a compressed form that is optimised for speed and provides 3-bit precision on the inter-strip position of the clusters using a simple weighted pulse height algorithm. For offline use a higher precision calculation, and an estimate of the uncertainty on this position, is provided [48]. This calculation uses the inter-strip pitch and track angle as well as the pulse-height of the strips in the cluster.

In addition to the standard output data format of the TELL1, a number of other output formats are provided for calibration and monitoring purposes and are decoded in the software framework. Notably, these include a non zero-suppressed raw data format, where the ADC values (at 8-bit precision) are provided for all strips.

A bit-perfect emulation of the full TELL1 processing algorithm is available in the software framework. Using the emulation the raw data can be processed to produce the cluster format. The performance of the TELL1 algorithms can thus be assessed at each stage of the processing. The emulation is also used to tune the optimal settings of the adjustable parameters in the TELL1, such as the signal thresholds used in the clustering algorithm.

A simulation framework for the VELO has been provided. This framework describes the material and layout of the VELO detector. The response of the silicon detectors and front-end chip pulse shape to the passage of particles is described, based on physically motivated parametrisations tuned to describe laboratory and test beam data. The resulting simulated analog signals are passed through the emulated TELL1 clustering algorithm and the output clusters are stored in the same format as for the real data [49].

\section{Monitoring}

The VELO data monitoring can be divided into two strands: the short term operational checks performed on-line; and the longer-term off-line performance monitoring.

The on-line monitoring is performed using the LHCb monitoring farm. Cluster and track monitoring, including monitoring of residuals for the alignment, is performed using the standard output data. Raw data is also produced for a subset of events at a rate of a fraction of a $\mathrm{Hz}$ and, through use of a special calibration trigger, read out to the monitoring farm. This rate allows the performance of individual channels to be accurately assessed on a timescale of one hour. The full information of the TELL1 processing boards is available through monitoring using the TELL1 credit-card PC. Preliminary tests of the on-line monitoring have already been performed in the VELO test beam.

A critical element of the monitoring is the determination of the beam-position. The alignment framework, reconstruction, pattern recognition, track fitting and vertex-finding algorithms are used to build up a 3 dimensional picture of the beam position. This monitoring process is used to determine the correct step-wise movements that are required to close the two halves and centre 
them around the beam at the start of an LHC fill. The beam stability is then monitored during LHC operation.

The off-line monitoring uses a range of analyses to assess the performance and to tune operational parameters including the high voltage applied to each sensor, the TELL1 hit processing parameters, and the cluster resolution model used in the tracking. The analyses include: time alignment studies for beam synchronisation; charge collection efficiency and signal-to-noise studies; resolution studies as a function of detector pitch and projected angle; cross-coupling, pedestal, noise and common-mode noise studies making particular use of the TELL1 emulation. These studies use the full range of VELO TELL1 output formats for the data and also make use of special calibration trigger and test-pulses generated in the Beetle front-end chip of the VELO modules.

\section{Alignment}

The alignment of the VELO relies on three components: the precision construction and assembly of the hardware; the metrology of the individual modules and assembled system; and the software alignment of the system using tracks.

The construction and assembly of the system is reported elsewhere in this section. The construction precision has tight mechanical tolerances, for example the silicon sensors are nominally located only $1 \mathrm{~mm}$ from the the aluminium RF foil. However, the driving factor for the required construction tolerance is the successful operation of the LHCb trigger. The VELO pattern recognition algorithm, used to identify high impact parameter tracks in the trigger, is performed for speed reasons initially in the $r z$ projection. This requires that the strips on the R-sensors accurately describe circles around the beam position.

A survey of the individual modules and of the assembled halves on the module supports was performed. The relative positions of the modules and of the R- and $\phi$-sensors on a single module were measured to a precison of better than $10 \mu \mathrm{m}$. The silicon sensors were found to have no significant curvature: 8 points were measured on the surface of the silicon sensors and the mean RMS deviation of the sensors from a plane was found to be $14 \mu \mathrm{m}$. The deviations of the modules from their nominal positions are shown in figure 5.14 for the two detector halves (A and C sides). The scatters give an impression of the total assembly precision. In the $x$ coordinate the scatter is very small, and the overall offsets are fully compensated by the fact that the two halves move independently in $x$. In $y$ there is a small slope seen in the C-side results, which was traced to the fact that the module support was not perfectly flat, but had a $40 \mu \mathrm{m}$ corkscrew twist between the corners. The resulting slope was fully compensated by adjustments of the detector supports during the installation of the halves.

The precision survey is an important element of the alignment: not only does it provide the starting position for the VELO software alignment but it also constrains degrees of freedom of the system which will not be possible to accurately measure with data. For example, the overall $z$ positions of the sensors are obtained from the metrology. The sensor alignment parameters obtained from the survey were propagated to the LHCb conditions database.

Whilst the survey was performed at room temperature and pressure, no significant deviations are expected for the final system. The module support will be maintained at a constant temperature of $20^{\circ} \mathrm{C}$. The deviations of the system under vacuum have been determined from the software 

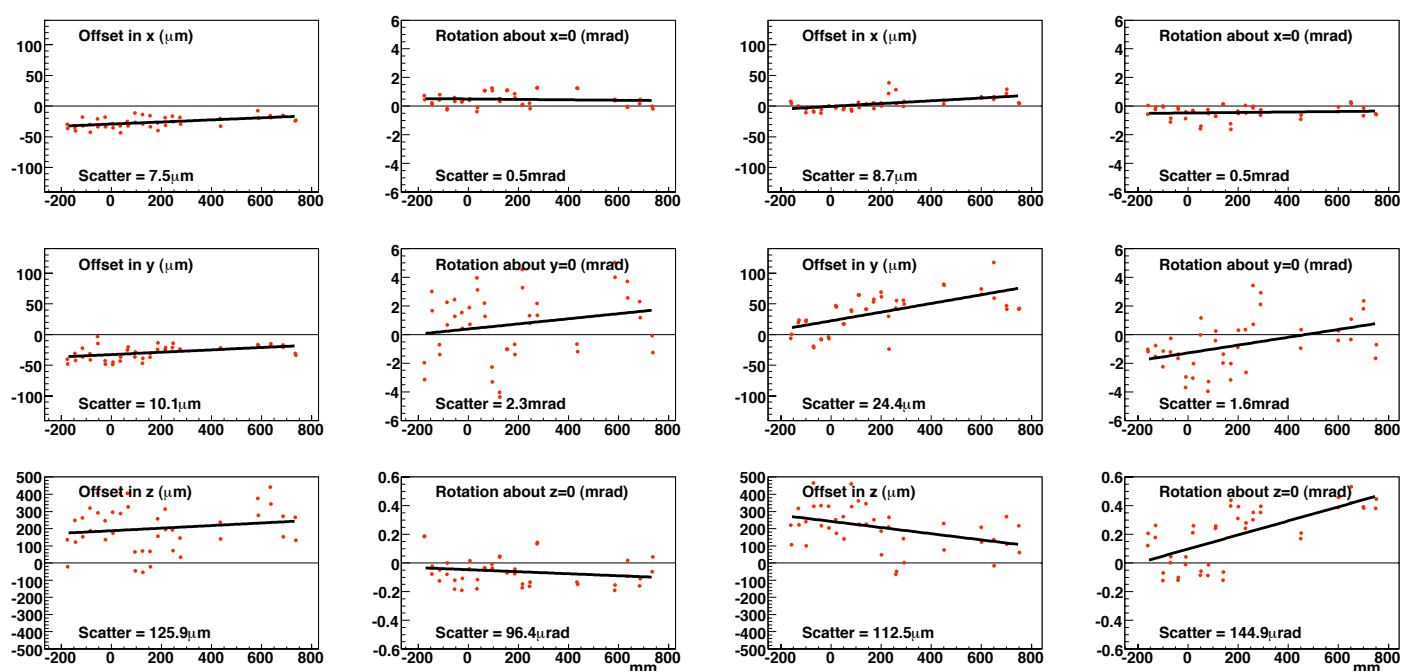

Figure 5.14: Results of the metrology of the fully mounted VELO halves vs $z$. The deviations from nominal are shown for every sensor. The six plots on the left show the result for the A side, and on the right for the $\mathrm{C}$ side. The $r z$ tracking is sensitive to scatters and offsets in the $x$ coordinate (between modules) and $y$ coordinate (between modules, and between the detector halves).

alignment in a test beam using a partially assembled VELO and seen to be typically $10 \mu \mathrm{m}$ or less, as shown in figure 5.15 .

As previously stated, prior to the LHC establishing stable beams, the VELO is in a retracted position and is brought into its nominal position only after stable beam is established. The position of the two halves will be known through the motion control and position measurement system to an accuracy of $10 \mu \mathrm{m}$. Combining this information with the relative alignment of the modules obtained from the previous fill is expected to provide sufficient alignment accuracy for operation of the VELO trigger. However, the option to perform a software alignment at the start of each fill remains, should this prove necessary.

The software alignment procedure for the detector divides into four distinct parts:

- An alignment of the relative position of the $R$ and $\Phi$ sensors on each module using the shape of the residual distributions across the sensors.

- An alignment of the modules within each VELO-half box using the residuals of hits on reconstructed tracks.

- A relative alignment of the two half-boxes with respect to each other principally relying on using tracks passing through the geometrical overlap between the modules in the two halfboxes.

- A global alignment of all sub-detectors relative to each other. This part is reviewed in [50]. 

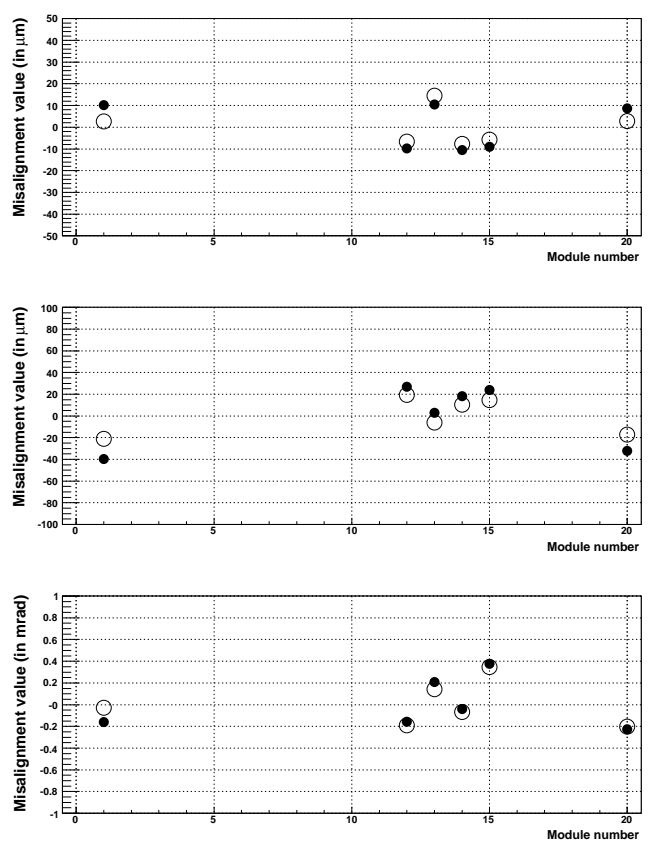

Figure 5.15: $x$-axis translation alignment constants of the VELO modules determined by software alignment from data recorded during the VELO test beam in air (open circles) and in vacuum (solid points). The modules are seen to be stable in the different conditions, including different thermal gradients across the modules.

\section{Relative sensor alignment}

The R- and $\phi$-sensors bonded in an individual module have had their relative positions measured during the system metrology. To further improve and monitor their relative misalignment a method has been developed that exploits distortions observed in the residuals across the sensor. It can be shown that the relation between residuals $\left(\varepsilon_{\mathrm{R} / \Phi}\right)$ and misalignments in the sensor plane $\left(\Delta_{x}, \Delta_{y}, \Delta_{\gamma}\right)$ is given by

$$
\left\{\begin{array}{llll}
\varepsilon_{\mathrm{R}}=-\Delta_{x} \cos \phi_{\text {track }} & +\Delta_{y} \sin \phi_{\text {track }} & & (\mathrm{R} \text { sensor }) \\
\varepsilon_{\Phi}=+\Delta_{x} \sin \phi_{\text {track }} & +\Delta_{y} \cos \phi_{\text {track }} & +\Delta_{\gamma} r_{\text {track }} & (\Phi \text { sensor })
\end{array},\right.
$$

where $r_{\text {track }}$ and $\phi_{\text {track }}$ are the radius and the azimuthal angle of the extrapolated track position, respectively. The misalignments $\Delta_{x}, \Delta_{y}$ are determined by a fit to the shape of the residual distribution as a function of the azimuthal angle, while $\Delta_{\gamma}$, the rotation misalignment around the $z$-axis, is determined by a fit to the shape of the residual distribution as a function of the radius. The optimal alignment can be found after three iterations of this procedure, using the previously determined alignment constants as input to the next iteration.

The method has been tested using MonteCarlo simulations and VELO test beam data. It has been shown that an alignment precision at the $\mu \mathrm{m}$ level can be achieved for $\Delta_{x}$ and $\Delta_{y}$, while $\Delta_{\gamma}$ will be determined to higher precision by the module alignment algorithm. 


\section{Module alignment and VELO half alignment}

The module alignment and the VELO half alignment are dependent on the same approach, a noniterative method using matrix inversion. The alignment is based upon a $\chi^{2}$ function produced from the residuals between the tracks and the measured clusters. The track and alignment parameters can be obtained through minimisation of this $\chi^{2}$ function.

The equations which describe the trajectories of particles are expressed as a linear combination of both the local (track-dependent) parameters and the global (alignment) parameters. All tracks are correlated since the global alignment parameters are common to each track, hence it is necessary to fit all tracks simultaneously.

The $\chi^{2}$ function can be minimised by solving the set of simultaneous equations given by the derivatives of the $\chi^{2}$ with respect to the local track parameters and global alignment parameters. This results in a system of equations of a final size, $n_{\text {total }}$ given by:

$$
n_{\text {total }}=n_{\text {local }} \times n_{\text {tracks }}+n_{\text {global }}
$$

where $n_{\text {local }}$ is the number of local parameters per track (four parameters for a straight line in $3 \mathrm{D}), n_{\text {tracks }}$ is the number of tracks used for the alignment and $n_{\text {global }}$ is the number of alignment constants. Whilst the direct inversion of such large matrices is not computationally practical, the alignment can be handled by inverting the matrix by partition, thus reducing the problem to a $n_{\text {global }} \times n_{\text {global }}$ matrix inversion. Inversion by partitioning is handled by the Millepede program [51].

The number of tracks required for an effective alignment is relatively modest but the alignment is improved by using a mixture of tracks from primary vertex interactions and a complementary track set from a source such as beam-halo particles. The relative alignment of the two half-boxes is primarily constrained by tracks that pass through both halves. However, when the VELO is retracted an alternative technique is required which relies upon fitting primary vertices using tracks fitted in both halves of the VELO. The CPU requirements of the alignment are also low: of order minutes on a single PC.

Tests using data from the VELO test beam and on MonteCarlo simulation have demonstrated that $x$ and $y$-axis translations of the modules can be constrained at the few $\mu \mathrm{m}$ level and rotations around the $z$ axis of order $0.1 \mathrm{mrad}$, with weaker sensitivity obtained to the other degrees of freedom. MonteCarlo simulation tests have demonstrated that $x$ and $y$ translations of the half-boxes can be constrained at better than $20 \mu \mathrm{m}$ and rotations around these axes to better than $0.1 \mathrm{mrad}$. Rotations around the $z$-axis are constrained at the $0.2 \mathrm{mrad}$ level.

\subsubsection{VELO performance}

The VELO layout has been optimised to minimise the amount of material in the acceptance while providing good geometrical coverage. All tracks inside the LHCb acceptance $(1.6<\eta<4.9)$ pass through at least three modules, as shown in figure 5.16.

The individual hit resolution of the sensors has been determined in a test beam and is a strong function of the sensor pitch and projected angle (the angle perpendicular to the strip direction), as shown in figure 5.17. The best raw resolution obtained is $7 \mu \mathrm{m}$. As shown in figure 5.18 perfor- 

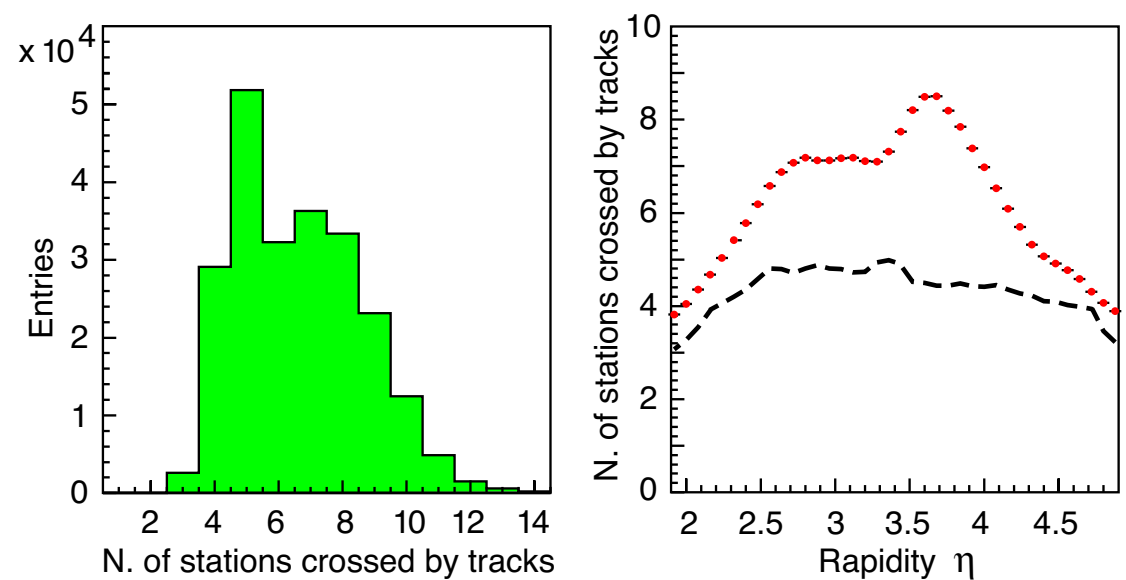

Figure 5.16: The left plot shows the number of stations hit per track in the VELO and the right plot shows the number of hits of a track in the VELO modules as a function of the pseudorapidity of the track. The dashed line indicates the limit above which $95 \%$ of the tracks lie.
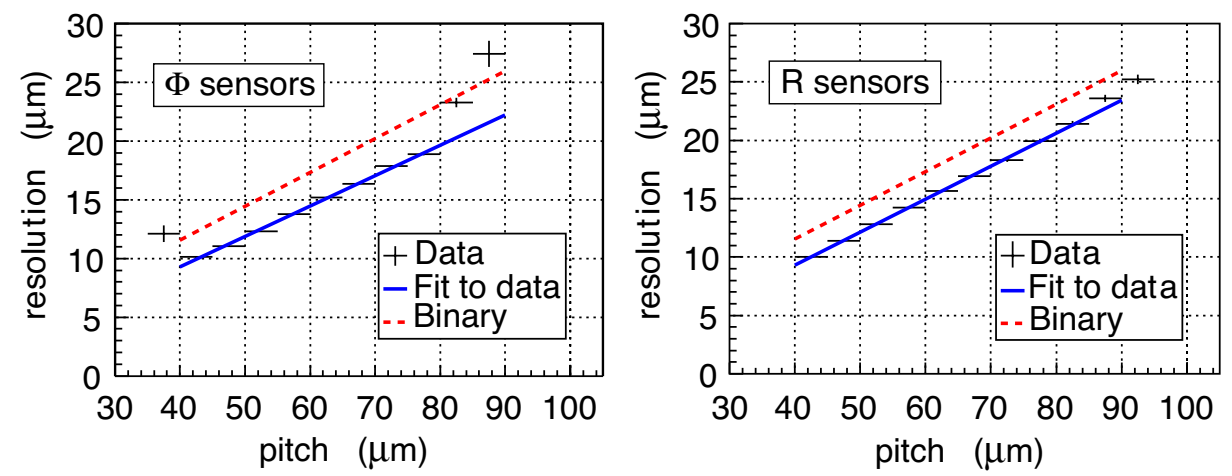

Figure 5.17: The raw hit resolution as a function of strip pitch as measured in the test beam for particles of normal incidence. The dashed line indicates the resolution expected for digital readout. The data points show the resolution as measured from the weighted centre of the charges on the strips.

mance improves for the low angle tracks when imperfections in the weighted charge distribution between two strips are taken into account [52].

In addition, crosstalk originating from inter-strip coupling, from coupling between electronic channels, and from signal feed-forward and backward in the analog transmission have not been taken into account. Once these have been fully parametrised further improvement in the resolution obtained from the system is anticipated. 


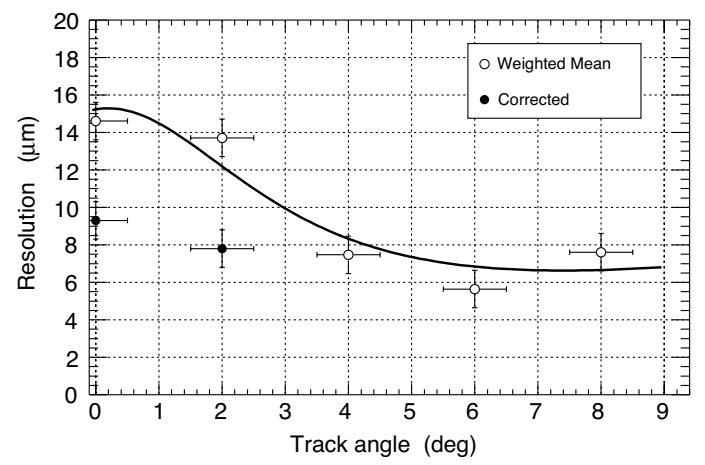

Figure 5.18: The individual hit resolution of a VELO R-sensor as a function of the projected angle for a pitch of $85 \mu \mathrm{m}$. The open circles show the resolution as derived from a weighted mean. The filled circles include the corrections for the imperfections in the weighted charge distribution between two strips.

\subsection{Silicon Tracker}

The Silicon Tracker (ST) comprises two detectors: the Tracker Turicensis ${ }^{10}$ (TT) $[2,53]$ and the Inner Tracker (IT) [54]. Both TT and IT use silicon microstrip sensors with a strip pitch of about $200 \mu \mathrm{m}$. The TT is a $150 \mathrm{~cm}$ wide and $130 \mathrm{~cm}$ high planar tracking station that is located upstream of the LHCb dipole magnet and covers the full acceptance of the experiment. The IT covers a $120 \mathrm{~cm}$ wide and $40 \mathrm{~cm}$ high cross shaped region in the centre of the three tracking stations downstream of the magnet. Each of the four ST stations has four detection layers in an $(x-u-v-x)$ arrangement with vertical strips in the first and the last layer and strips rotated by a stereo angle of $-5^{\circ}$ and $+5^{\circ}$ in the second and the third layer, respectively. The TT has an active area of about $8.4 \mathrm{~m}^{2}$ with 143360 readout strips of up to $38 \mathrm{~cm}$ in length. The IT has an active area of $4.0 \mathrm{~m}^{2}$ with 129024 readout strips of either $11 \mathrm{~cm}$ or $22 \mathrm{~cm}$ in length.

The main design choices for the Silicon Tracker detectors were largely driven by the following considerations:

Spatial resolution. Simulation studies have demonstrated that a single-hit resolution of about $50 \mu \mathrm{m}$ is adequate for both the TT and the IT. The momentum resolution of the spectrometer is then dominated by multiple scattering over almost the full range of particle momenta. Readout strip pitches of about $200 \mu \mathrm{m}$ meet this requirement and were therefore chosen for both detectors.

Hit occupancy. Charged particle densities of about $5 \times 10^{-2}$ per $\mathrm{cm}^{2}$ for minimum bias events are expected in the innermost regions of the TT. They fall off by two orders of magnitude to about 5 $\times 10^{-4}$ per $\mathrm{cm}^{2}$ in the outermost regions of the detector. Different readout strip lengths were chosen for different regions of the detector to keep maximum strip occupancies at the level of a few percent while minimizing the number of readout channels. For the IT, expected charged-particle densities for minimum-bias events range from about $1.5 \times 10^{-2}$ per $\mathrm{cm}^{2}$ close to the LHC beampipe to 2 $\times 10^{-3}$ per $\mathrm{cm}^{2}$ in the outer regions of the detector. Also here, the chosen strip geometries result in maximum strip occupancies that do not exceed a few percent.

\footnotetext{
${ }^{10}$ The Tracker Turicensis was formerly known as the Trigger Tracker.
} 
Signal shaping time. In order to avoid pile-up of events from consecutive LHC bunch crossings, fast front-end amplifiers with a shaping time of the order of the bunch crossing interval of $25 \mathrm{~ns}$ have to be used. The benchmark parameter is the remaining fraction of the signal amplitude at the sampling time of the subsequent bunch crossing, $25 \mathrm{~ns}$ after the maximum of the pulse. Simulation studies have shown that signal remainders of 50\% for the TT and 30\% for the IT are acceptable for the track reconstruction algorithms.

Single-hit efficiency. Each detection layer should provide full single-hit efficiency for minimum ionising particles while maintaining an acceptably low noise hit rate. The critical parameter is the signal-to-noise ratio, defined as the most probable signal amplitude for a minimum ionising particle divided by the RMS of the single strip noise distribution. Test beam studies have shown that the hit efficiency starts to decrease rapidly as the signal-to-noise ratio drops below 10:1. The detector was designed such that a signal-to-noise ratio in excess of 12:1 can be expected taking into account the expected deterioration from radiation damage corresponding to ten years of operation at nominal luminosity.

Radiation damage. For ten years of operation at nominal luminosity, expected $1 \mathrm{MeV}$ neutron equivalent fluences in the innermost regions of the detectors do not exceed $5 \times 10^{14}$ per $\mathrm{cm}^{2}$ for the TT and $9 \times 10^{12}$ per $\mathrm{cm}^{2}$ for the IT. Basic design rules for radiation hard silicon sensors were followed to ensure that the detectors will survive these fluences. The detector was designed and tested to work at bias voltages of up to $500 \mathrm{~V}$. The sensors need to be operated at a temperature of $5^{\circ} \mathrm{C}$ or lower to suppress radiation damage induced leakage currents to a level where (a) shot noise does not significantly deteriorate the signal-to-noise performance of the detector and (b) the risk of thermal runaway due to the power dissipated by the leakage currents is avoided.

Material budget. As the momentum resolution of the $\mathrm{LHCb}$ spectrometer is dominated by multiple scattering, the material budget of the detectors had to be kept as small as possible. The TT was designed such that front-end readout electronics and mechanical supports are located outside of the LHCb acceptance. This was not possible in the case of the IT, which is located in front of the active region of the OT detectors. Here, a significant design effort was made to keep the amount of material for mechanical supports and for the cooling of front-end electronics as small as possible.

Number of readout channels. Readout electronics are a major contribution to the overall cost of the detector. The largest readout pitches compatible with the required spatial resolution and the longest readout strips compatible with requirements on occupancy and signal-to-noise performance were chosen in order to minimize the number of readout channels.

Different constraints on the detector geometries resulted in different designs for the detector modules and station mechanics of the TT and the IT. These are described in sections 5.2.1 and 5.2.2, respectively. Common to both parts of the ST are the readout electronics, the power distribution, and the detector control and monitor systems. These are the topic of section 5.2.3. Finally, the expected detector performance, based on test beam measurements and simulation studies, is discussed in section 5.2.4.

\subsubsection{Tracker Turicensis}

All four detection layers of the TT are housed in one large light tight and thermally and electrically insulated detector volume, in which a temperature below $5^{\circ} \mathrm{C}$ is maintained [55]. The detector 


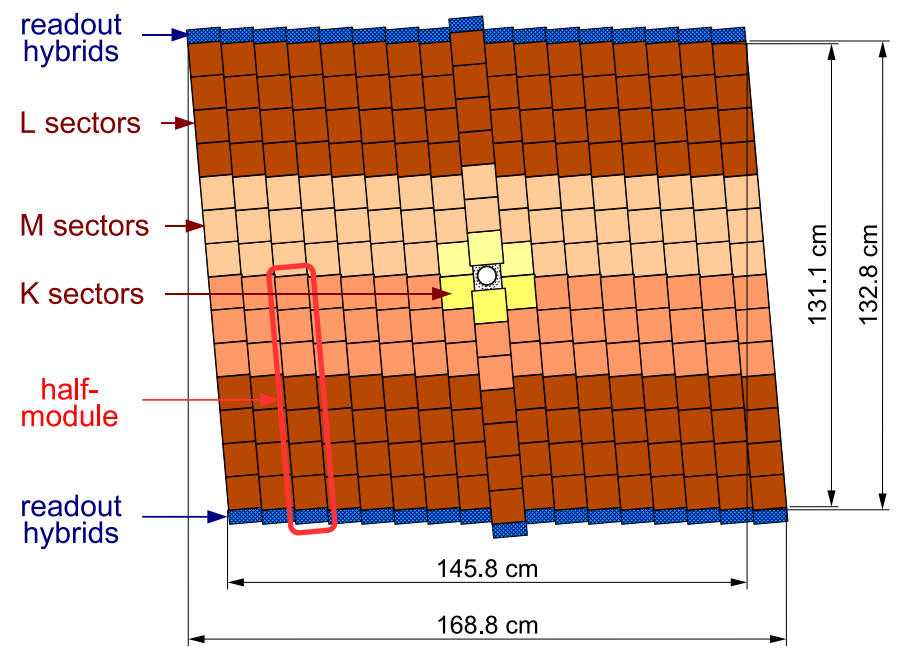

Figure 5.19: Layout of the third TT detection layer. Different readout sectors are indicated by different shadings.

volume is continuously flushed with nitrogen to avoid condensation on the cold surfaces. To aid track reconstruction algorithms, the four detection layers are arranged in two pairs, $(x, u)$ and $(v, x)$, that are separated by approximately $27 \mathrm{~cm}$ along the LHC beam axis.

The layout of one of the detection layers is illustrated in figure 5.19. Its basic building block is a half module that covers half the height of the LHCb acceptance. It consists of a row of seven silicon sensors organized into either two or three readout sectors. The readout hybrids for all readout sectors are mounted at one end of the module. The regions above and below the LHC beampipe are covered by one such half module each. The regions to the sides of the beampipe are covered by rows of seven (for the first two detection layers) or eight (for the last two detection layers) 14sensor long full modules. These full modules cover the full height of the LHCb acceptance and are assembled from two half modules that are joined together end-to-end. Adjacent modules within a detection layer are staggered by about $1 \mathrm{~cm}$ in $z$ and overlap by a few millimeters in $x$ to avoid acceptance gaps and to facilitate the relative alignment of the modules. In the $u$ and $v$ detection layers, each module is individually rotated by the respective stereo angle.

A main advantage of this detector design is that all front-end hybrids and the infrastructure for cooling and module supports are located above and below the active area of the detector, outside of the acceptance of the experiment.

\section{TT detector modules}

The layout of a half module is illustrated in figure 5.20. It consists of a row of seven silicon sensors with a stack of two or three readout hybrids at one end. For half modules close to the beampipe, where the expected particle density is highest, the seven sensors are organized into three readout sectors (4-2-1 type half modules).

For the other half modules, the sensors are organized into two readout sectors (4-3 type half modules). In both cases, the first readout sector ( $\mathrm{L}$ sector) is formed by the four sensors closest to 


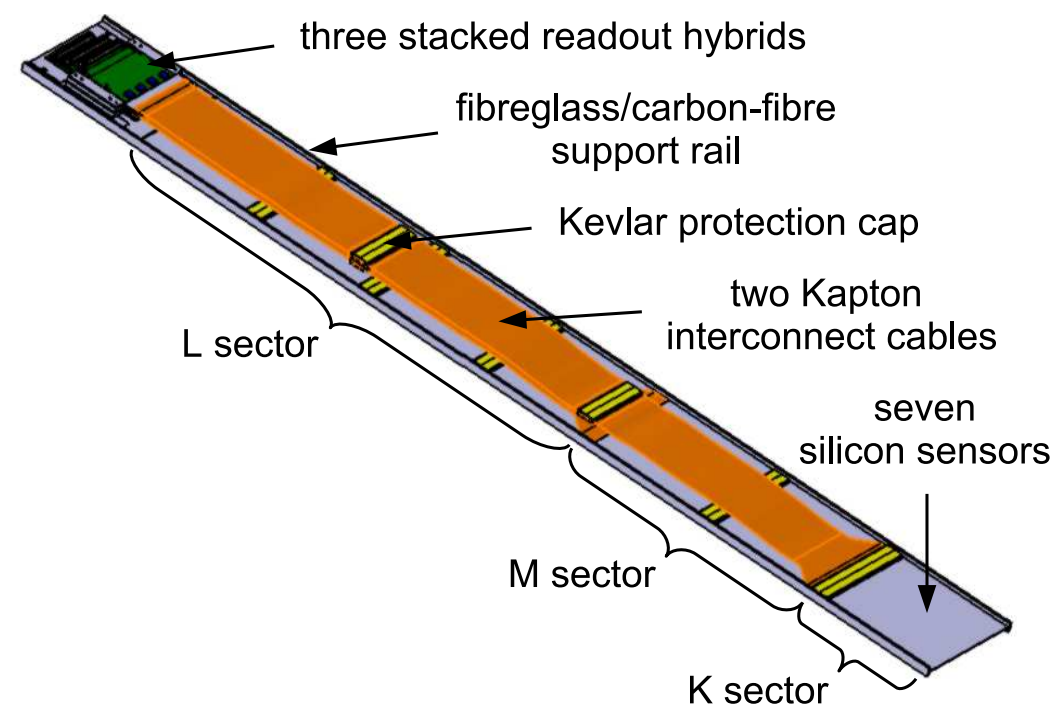

Figure 5.20: View of a 4-2-1 type TT detector module.

the readout hybrids and furthest away from the beam. The strips of the four sensors are bonded together and directly connected to the lower-most readout hybrid. For 4-3 type half modules, the strips of the remaining three sensors are bonded together and form the second readout sector (M sector). They are connected via a $39 \mathrm{~cm}$ long Kapton flex cable (interconnect cable) to a second readout hybrid mounted on top of the L hybrid. For 4-2-1 type half modules, the three remaining sensors are subdivided into an intermediate two sensor sector ( $\mathrm{M}$ sector) and a third sector consisting of the single sensor closest to the beam (K sector). The two readout sectors are connected via $39 \mathrm{~cm}$ respectively $58 \mathrm{~cm}$ long Kapton interconnect cables to two separate front-end hybrids that are mounted on top of the $\mathrm{L}$ hybrid. Bias voltage is provided to the sensor backplanes via a thin Kapton flex cable that runs along the back of the half module. The half module is mechanically held together by two thin fibreglass/carbon fibre rails that are glued along the edges of the L hybrid and the seven silicon sensors.

Silicon sensors. The silicon sensors for the TT are $500 \mu \mathrm{m}$ thick, single sided $p^{+}$-on- $n$ sensors. ${ }^{11}$ They are $9.64 \mathrm{~cm}$ wide and $9.44 \mathrm{~cm}$ long and carry 512 readout strips with a strip pitch of $183 \mu \mathrm{m}$.

Kapton interconnect cables. The Kapton interconnect cables for the $\mathrm{M}$ and $\mathrm{K}$ readout sectors were produced using standard plasma-etching technology. ${ }^{12}$ They carry 512 signal strips and two pairs of bias voltage and ground strips on a $100 \mu \mathrm{m}$ thick Kapton substrate. The strips consist of $7 \mu \mathrm{m}$ thick copper with a $1 \mu \mathrm{m}$ thick gold plating, are $15 \mu \mathrm{m}$ wide and have a pitch of $112 \mu \mathrm{m}$. A short pitch-adapter section in which the strip pitch widens to $180 \mu \mathrm{m}$ permits to directly wirebond the strips on the cable to the silicon sensor strips. A copper mesh backplane provides a solid ground connection and shielding against pick-up noise. The small strip width was required to keep the strip capacitance of the cable small, but led to an unacceptably low production yield for fault

\footnotetext{
${ }^{11}$ The sensors are identical in design to the OB2 sensors used in the Outer Barrel of the CMS Silicon Tracker [56] and were produced by Hamamatsu Photonics K.K., Hamamatsu City, Japan.

${ }^{12}$ The Kapton interconnect cables were produced by Dyconex AG, Bassersdorf, Switzerland.
} 
free cables of the required length. The $39 \mathrm{~cm}$ long cables for the $\mathrm{M}$ sectors therefore had to be assembled from two shorter pieces, and the $58 \mathrm{~cm}$ long cables for the $\mathrm{K}$ sectors were assembled from three pieces. The pieces were joined together end-to-end by gluing them onto a thin strip of fibreglass reinforced epoxy. An electrically conductive adhesive tape was used to provide the electrical connection between the copper mesh backplanes of the two cable pieces. The signal, bias voltage and ground strips on the strip side of the cables were joined together by wire bonds.

Kevlar caps. Small Kevlar caps protect the wire bond rows on the strip side of the Kapton interconnect cables as well as those between silicon sensors. These caps are glued onto the surface of the cable or the sensors using standard two component epoxy glue. ${ }^{13}$

Readout hybrids. The front-end readout hybrids [57] consist of a carrier plate, a pitch adapter, and a four layer Kapton flex circuit that carries four front-end readout chips, some passive SMD components and an 80-pin board-to-board connector. ${ }^{14}$ Through this connector, the multiplexed analog detector signals are read out and the control signals, low voltage and bias voltage are provided to the half module. Two of the four conductive layers of the flex circuit are used for digital and analog power and ground. The other two layers carry the signal and control lines. Due to mechanical constraints, three variants of the readout hybrid are used for the three different types of readout sectors. The Kapton flex circuits for all three variants are identical except for a different overall length. The upper hybrids have to be shorter than the lower ones to make the readout connectors on all hybrids accessible when these are mounted on top of each other. The carrier plates give mechanical stability to the hybrid and act as a heat sink for the heat produced by the front-end chips. The pitch adapter matches the input pitch of the front-end chips to the pitch of the silicon sensors in case of the L hybrid and to the pitch of the Kapton interconnect cable in case of the $\mathrm{K}$ and $\mathrm{M}$ hybrids. For these, the carrier plate is made from gold plated copper and the pitch adapter is a rectangular piece of alumina $\left(\mathrm{Al}_{2} \mathrm{O}_{3}\right)$ substrate with strip lines produced using standard thin film technology. The pitch adapter is glued onto the carrier plate together with the Kapton flex circuit. The carrier plate for the $\mathrm{L}$ hybrid is a much more complicated piece that consists of aluminium nitride substrate and combines several functionalities. ${ }^{15}$ First of all, it carries not only the strip lines of the pitch adapter for the L sector, produced using standard thick film technology, but also additional traces and vias that serve to connect the bias voltage for all readout sectors. Next, the support rails of the detector module are glued along the sides of this carrier plate. And finally, this carrier plate provides the mechanical and thermal interface of the half module to the detector station. All these functionalities will be described in more detail below. Aluminium nitride was chosen as the material for this carrier plate because of its high thermal conductivity and its small thermal expansion coefficient that is reasonably well matched to that of the silicon sensors.

The $\mathrm{K}$ and $\mathrm{M}$ hybrids are mounted on top of the carrier plate of the L hybrid using $2.5 \mathrm{~mm}$ thick spacers made of copper. These ensure good thermal contact between the carrier plates of the $\mathrm{K}$ and $\mathrm{M}$ hybrids and the one of the $\mathrm{L}$ hybrid.

Support rails. The two half module support rails are $5.5 \mathrm{~mm}$ high and consist of a $1 \mathrm{~mm}$ thick strip of carbon fibre glued to a $1 \mathrm{~mm}$ thick strip of fibreglass reinforced epoxy. A small groove

\footnotetext{
${ }^{13}$ Araldite 2011 (AW106/HV953U), by Huntsman Advanced Materials, Basel, Switzerland.

${ }^{14}$ The flex circuits were produced by Optiprint AG, Berneck, Switzerland.

${ }^{15}$ The production of the pitch adapters for the $\mathrm{K}$ and $\mathrm{M}$ hybrids and of the aluminium nitride carrier plate for the L hybrids as well as the assembly and bonding of all hybrids was done by RHe Microsystems, Radeberg, Germany.
} 


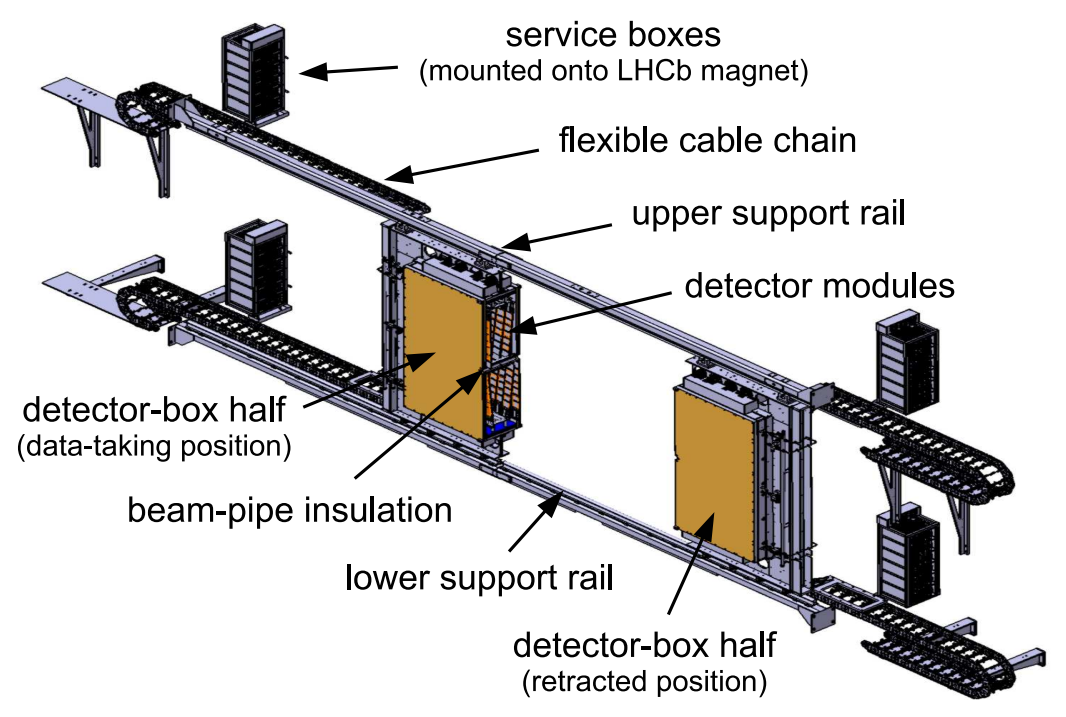

Figure 5.21: View of the TT station mechanics.

is milled into the flat side of the fibreglass strip which permits to slide the rail over the edges of the seven silicon sensors and the carrier plate of the L hybrid. The rail is glued to these using standard two component epoxy glue. The rôle of the fibreglass strip is to ensure the necessary electrical insulation between the strip side of the silicon sensors (which are at ground potential) and their backplanes (which carry the bias voltage of up to $500 \mathrm{~V}$ ). The mechanical rigidity of the rail is defined mainly by the carbon fibre strip. The fibreglass strip spans the full length of the half module but the carbon fibre strip ends at the fourth silicon sensor from the hybrids. This permits to join two half modules to a 14-sensor long full module by gluing an additional carbon fibre strip to the free sections of fibreglass strip on both half modules.

Bias voltage. Bias voltage is supplied separately for each of the readout sectors on a half module via the cable that plugs into the corresponding front-end hybrid. From the hybrid it is connected to aluminium traces on the carrier plate of the $\mathrm{L}$ sector, using wire bonds in case of the $\mathrm{L}$ hybrid and thin copper wires in case of the $\mathrm{M}$ and $\mathrm{K}$ hybrids. From here, all bias voltages are brought to the back of the half module through aluminium vias that are embedded in the aluminium nitride substrate. Finally, a thin Kapton flex cable, which is glued along the back of the half module and carries one copper trace per readout sector, carries the bias voltage to the backplanes of the silicon sensors. The electrical connections between the Kapton flex cable and the sensor backplanes and between the cable and the bias voltage pads on the back of the aluminium nitride are made by wire bonding.

\section{TT detector station}

An isometric drawing of the detector station is shown in figure 5.21. It consists of two halves, one on each side of the LHC beampipe.

The half stations are mounted on rails and can be retracted horizontally for detector maintenance and bakeouts of the LHC beampipe. The main structural element of each half station is a $\mathrm{C}$-shaped aluminium frame. It carries a detector box that is made of light weight aluminium clad 
foam. This box is open on the side facing the beampipe such that the two half stations in data taking position define one large volume that contains all detector modules. Mounted against the top and bottom walls of the detector box are horizontal cooling plates that provide the mechanical support for the detector modules as well as cooling of the front-end hybrids and the detector volume. The cooling plates incorporate cooling pipes through which $\mathrm{C}_{6} \mathrm{~F}_{14}$ at $-15^{\circ} \mathrm{C}$ is circulated as a cooling agent. Additional cooling elements are mounted vertically, close to the outer side walls of the detector volume. All electrical signals (detector signals, control signals and supply voltages) are transmitted on Kapton-flex cables through specially designed feedthroughs in the top and bottom walls of the detector box.

Detector box. The C-shaped support frames are assembled from $15 \mathrm{~mm}$ thick aluminium plates. They rest on a lower precision rail and are guided by an upper precision rail. These two rails are aligned parallel to each other to better than $100 \mu \mathrm{m}$ in order to avoid possible distortions of the C-frame during insertion or retraction. Mounted flat against the inner surfaces of the Cframes are stiff sandwich plates consisting of a $30 \mathrm{~mm}$ thick aramid honeycomb structure with $1 \mathrm{~mm}$ thick aluminium cladding. These sandwich plates define the outer walls of the detector box. The front and rear walls of the box are made of $40 \mathrm{~mm}$ thick panels of polyetherimide foam ${ }^{16}$ that are laminated on both sides with $25 \mu \mathrm{m}$ of aluminium for electrical insulation and $275 \mu \mathrm{m}$ of Kevlar for mechanical protection. These panels are screwed against the outer walls of the box and can be easily removed for the installation and maintenance of detector modules. Similar plates of $40 \mathrm{~mm}$ thick aluminium and Kevlar clad polyetherimide foam are also mounted flat against the inner surfaces of the outer walls of the box to improve thermal insulation here. Finally, the detector volume is closed off around the LHC beampipe by two specially machined semicylindrical pieces that again consist of the same polyetherimide foam clad with aluminium and Kevlar. The wall thickness of these beampipe insulation pieces is $30 \mathrm{~mm}$, except for cutouts at the locations of the detection layers. Here, the wall thickness is reduced to about $5 \mathrm{~mm}$ to reduce the dead space in between the beampipe and the innermost detector modules. A clearance of $5 \mathrm{~mm}$ between the detector box and the beampipe had to be maintained to satisfy LHC safety demands.

Cooling plates. There are a total of four cooling plates, one for each quadrant of the detector. They are mounted horizontally onto pillars made of polyacetal (POM) that are fixed against the upper or the lower walls of the detector box. A drawing of a cooling plate is shown in figure 5.22. It is a precisely machined plate of $8 \mathrm{~mm}$ thick aluminium that measures $897 \mathrm{~mm}$ in $x$ and $348 \mathrm{~mm}$ in $z$. Machined into its outer surface are semicircular grooves into which two coiled aluminium cooling pipes with an outer diameter of $10 \mathrm{~mm}$ and a total length of about $3.5 \mathrm{~m}$ are glued. Its inner surface is machined to an overall flatness of better than $100 \mu \mathrm{m}$.

Cooling balconies. Mounted vertically against the flat inner surface of the cooling plate are the aluminium cooling balconies that provide the mechanical, thermal and electrical interface between cooling plate and detector modules. Precision holes and pins ensure the accurate positioning of the balconies on the cooling plate. The detector module is screwed onto the flat, vertical surface of the balcony, ensuring a large contact surface and therefore good thermal contact between the balcony and the aluminium nitride carrier plate of the half module. The correct positioning of the module is ensured by precision holes and pins in the balcony and laser cut holes in the aluminium

\footnotetext{
${ }^{16}$ Airex R82.60, by Gaugler \& Lutz oHG, Aalen-Ebnat, Germany.
} 


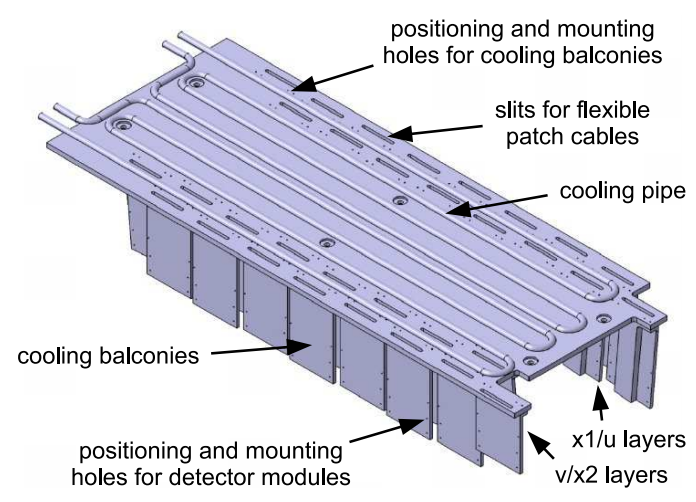

Figure 5.22: View of one TT cooling plate with mounted cooling balconies.

nitride carrier plate. There are two types of balconies, one for mounting modules vertically and one for mounting modules under an angle of $5^{\circ}$. Detector modules in the first two detection layers are mounted onto their balconies from the upstream side of the detector, those for the last two layers from the downstream side of the detector.

Cooling elements. The vertical cooling elements that are installed at both sides of the detector volume consist of $1 \mathrm{~mm}$ thick copper plates onto which long, coiled cooling ducts with a rectangular cross section are soldered.

Electrical connections. Detector signals are read out and control signals, low voltage and bias voltage supplied to the detector modules via $50 \mathrm{~cm}$ long Kapton flex cables. These cables pass through dedicated slits in the cooling plate and through specially designed feedthroughs in the top and bottom walls of the detector box. There is a separate Kapton flex cable for each readout sector. At one end it plugs directly into the board-to-board connector on the readout hybrid, at the other end it connects to a patch panel that is mounted on the outside of the detector box. From this patch panel, shielded twisted-pair cables lead through flexible cable chains to service boxes that are mounted against the front face of the $\mathrm{LHCb}$ dipole magnet. Here, the signals are prepared for digital optical transmission to the counting house as described in section 5.2.3. Each quadrant of the detector has a flexible cable chain and a stack of six service boxes.

\subsubsection{Inner Tracker}

Each of the three IT stations consists of four individual detector boxes that are arranged around the beampipe as shown in figure 5.23.

The detector boxes are light tight and electrically and thermally insulated, and a temperature below $5^{\circ} \mathrm{C}$ is maintained inside them. They are continuously flushed with nitrogen to avoid condensation on the cold surfaces. Each detector box contains four detection layers and each detection layer consists of seven detector modules. Adjacent modules in a detection layer are staggered by $4 \mathrm{~mm}$ in $z$ and overlap by $3 \mathrm{~mm}$ in $x$ to avoid acceptance gaps and facilitate the relative alignment of the modules. Detector modules in the boxes above and below the beampipe (top and bottom boxes) consist of a single silicon sensor and a readout hybrid. Detector modules in the boxes to the left and right of the beampipe (side boxes) consist of two silicon sensors and a readout hybrid. The resulting layout and dimensions of one of the IT detection layers are illustrated in figure 5.24. 


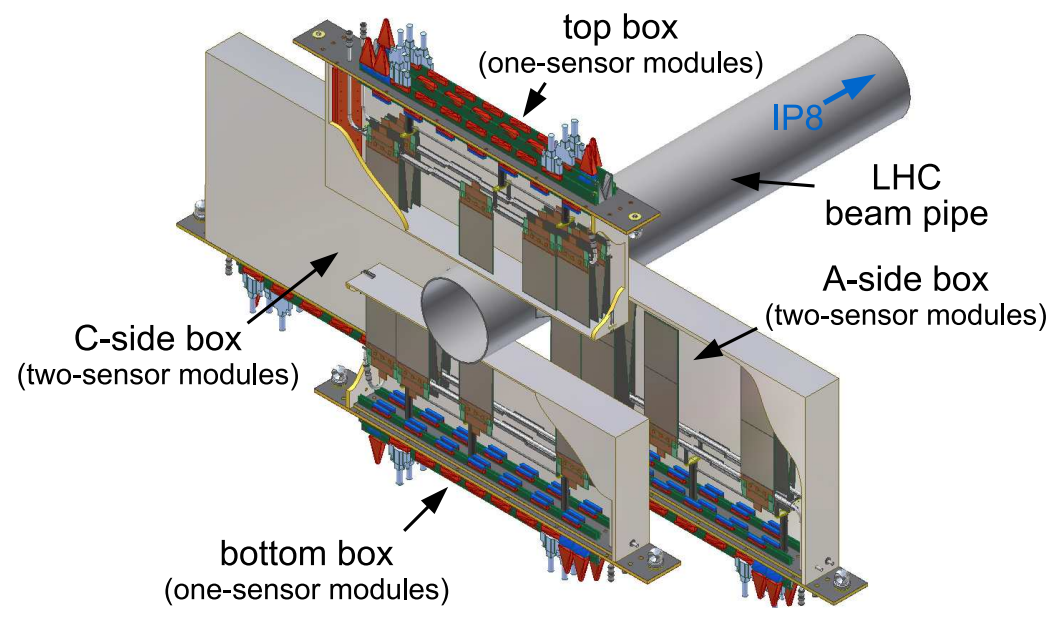

Figure 5.23: View of the four IT detector boxes arranged around the LHC beampipe.

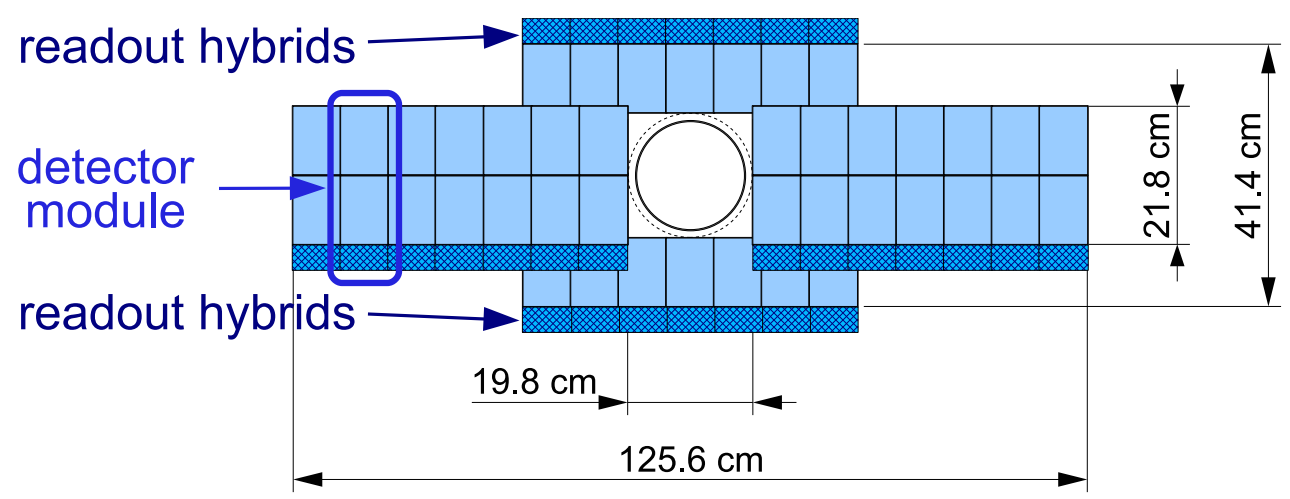

Figure 5.24: Layout of an $x$ detection layer in the second IT station.

\section{IT detector modules}

An exploded view of a detector module is shown in figure 5.25. The module consists of either one or two silicon sensors that are connected via a pitch adapter to a front-end readout hybrid. The sensor(s) and the readout hybrid are all glued onto a flat module support plate. Bias voltage is provided to the sensor backplane from the strip side through $n^{+}$wells that are implanted in the $n$ type silicon bulk. A small aluminium insert (minibalcony) that is embedded into the support plate at the location of the readout hybrid provides the mechanical and thermal interface of the module to the detector box.

Silicon sensors. Two types of silicon sensors of different thickness, but otherwise identical in design, are used in the IT. ${ }^{17}$ They are single-sided $p^{+}$-on- $n$ sensors, $7.6 \mathrm{~cm}$ wide and $11 \mathrm{~cm}$ long, and carry 384 readout strips with a strip pitch of $198 \mu \mathrm{m}$. The sensors for one-sensor modules are $320 \mu \mathrm{m}$ thick, those for two-sensor modules are $410 \mu \mathrm{m}$ thick. As explained in section 5.2.4 below, these thicknesses were chosen to ensure sufficiently high signal-to-noise ratios for each module type while minimising the material budget of the detector.

\footnotetext{
${ }^{17}$ The sensors were designed and produced by Hamamatsu Photonics K.K., Hamamatsu City, Japan.
} 


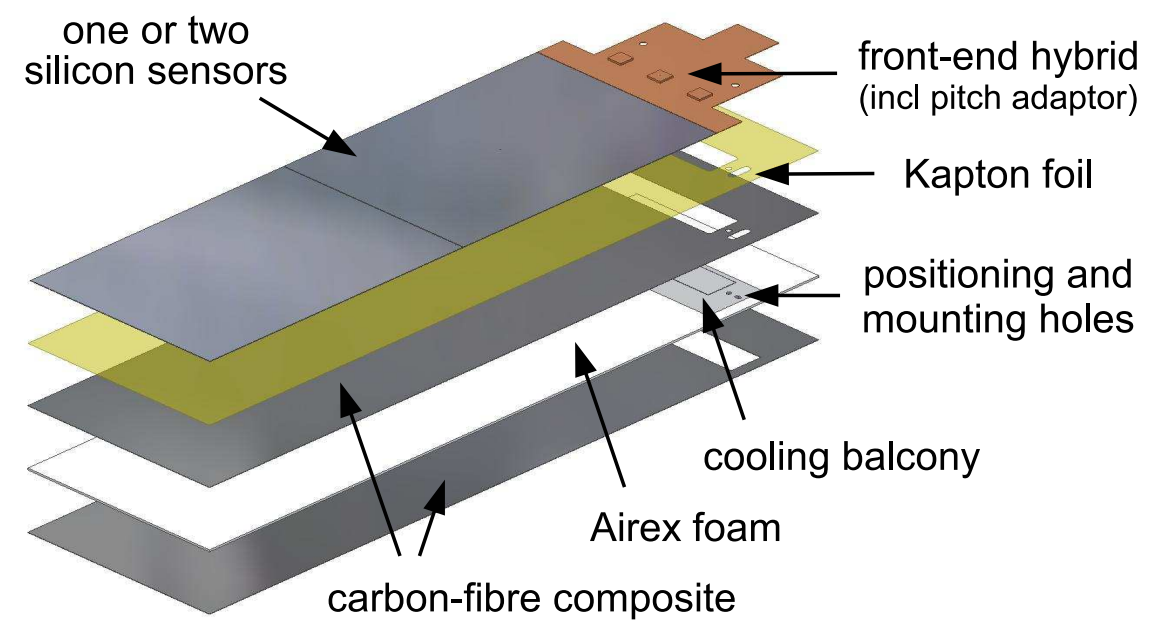

Figure 5.25: Exploded view of a two-sensor IT module. One-sensor modules are similar except that the support plate is shorter and carries only one sensor.

Readout hybrids. The IT front-end readout hybrids consist of a four layer Kapton flex circuit that is very similar in design and routing to that of the TT hybrids, and a pitch adapter that is similar to that used for the $\mathrm{M}$ and $\mathrm{K}$ hybrids of the TT. ${ }^{18}$ The only differences are (a) that the Kapton flex circuit for the IT carries only three front-end chips and (b) that it incorporates an $89 \mathrm{~mm}$ long readout tail with straight traces, at the end of which a 60-pin board-to-board connector is mounted. The pitch adapter is glued onto the Kapton flex circuit and the Kapton flex circuit is glued directly onto the module support plate.

Module support plate. The module support plate ${ }^{19}$ consists of a $1 \mathrm{~mm}$ thick sheet of polyetherimide foam ${ }^{20}$ sandwiched in between two $200 \mu \mathrm{m}$ thick layers of carbon fibre composite. The latter are produced from two layers of thermally highly conductive carbon fibres ${ }^{21}$ that are oriented at $\pm 10^{\circ}$ with respect to the module axis. A $25 \mu \mathrm{m}$ thick Kapton foil is laminated on top of the upper carbon fibre layer to electrically insulate it from the backplane of the silicon sensors, which carries the sensor bias voltage of up to $500 \mathrm{~V}$. The support plate extends on all sides by $1 \mathrm{~mm}$ over the edges of the silicon sensors to protect these mechanically. The edges of the support plate are sealed with a non conductive epoxide resin to prevent loose fibres from sticking out and touching the sensor, where they might cause a short between the strip side and the backplane. At the location of the front-end readout chips, a $70 \mathrm{~mm}$ wide, $15 \mathrm{~mm}$ high and $1.5 \mathrm{~mm}$ thick aluminium piece called minibalcony is inserted in the support plate. It is glued to the support plate using thermally conductive glue. ${ }^{22}$

Minibalcony. The minibalcony defines the mechanical and thermal interface of the module to the detector box. It is machined to a flatness of better than $30 \mu \mathrm{m}$ and contains precision holes for

\footnotetext{
${ }^{18}$ The production of flex prints and pitch adapters and the assembly of the readout hybrids took place at the same two companies as for the TT.

${ }^{19}$ The module support plates were produced by Composite Design, Echandens, Switzerland.

${ }^{20}$ Airex R82.60, by Gaugler \& Lutz oHG, Aalen-Ebnat, Germany.

${ }^{21}$ K13D2U, by Mitsubishi Chemical Corp., Tokyo, Japan.

${ }^{22}$ TRA-DUCT 2902, by TRA-CON Inc., Bedford, Massachusetts, USA.
} 


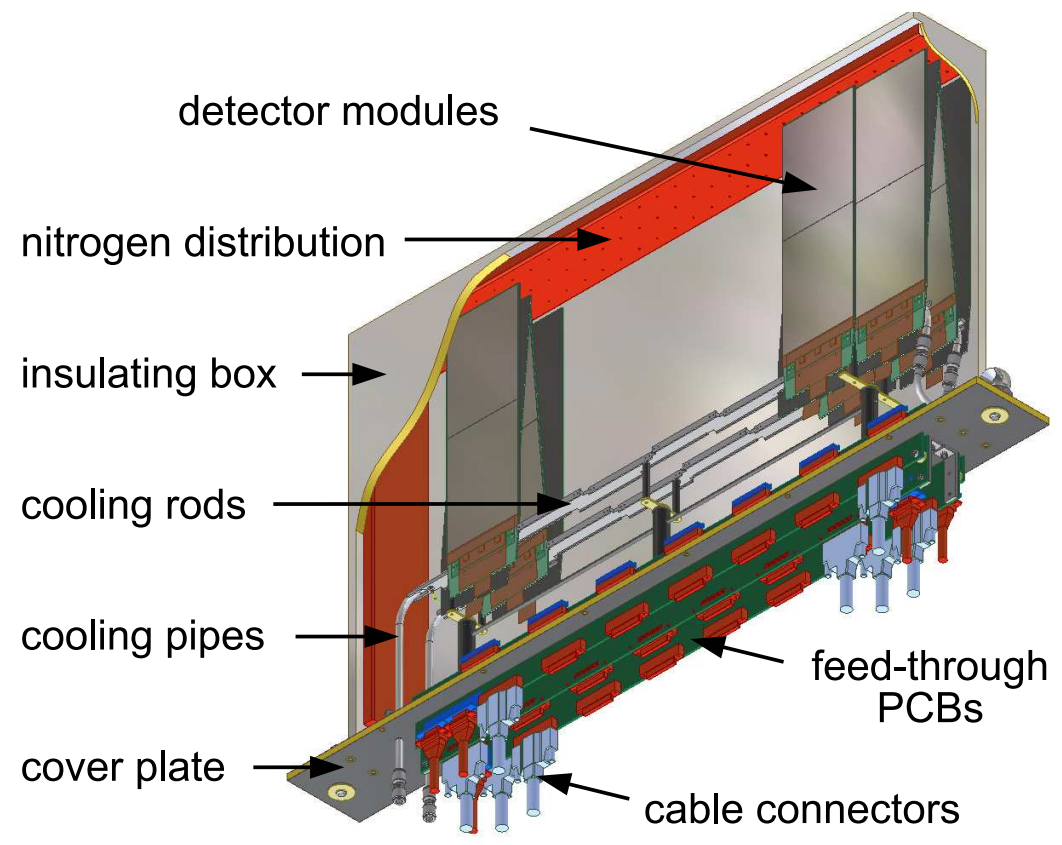

Figure 5.26: View of an IT side box. Top/bottom boxes are similar except that the box is shorter and contains one-sensor modules.

the mounting and exact positioning of the module. There are two types of minibalconies: one for modules that will be mounted vertically and another one for modules that will be mounted with the $5^{\circ}$ stereo angle. The minibalcony provides a direct heat path from the front-end chips to the cooling rod, onto which the modules are mounted as described below. It also thermally connects the carbon fibre sheets of the module support plate to the cooling rod. Due to their good thermal conductivity along the module axis and their large surface area, the module support plates therefore contribute to the cooling of the silicon sensors and the detector volume.

The silicon sensors are glued onto the module support plate using strips of silicone glue. ${ }^{23}$ The hybrids are glued onto the module support plate using standard two component epoxy glue. Small spots of thermally conductive glue ${ }^{24}$ are applied at the location of the front-end readout chips in order to improve the thermal contact between the chips and the minibalcony.

\section{IT detector boxes}

An isometric view of a detector box is shown in figure 5.26. Its main structural element is a cover plate, onto which two cooling rods are mounted. These cooling rods incorporate cooling pipes through which $\mathrm{C}_{6} \mathrm{~F}_{14}$ at $-15^{\circ} \mathrm{C}$ circulates as a cooling agent. Printed-circuit boards that are inserted vertically through the cover plate serve to transmit supply voltage and detector and control signals from and to the detector modules inside the box. The detector volume is closed by an insulating box that is assembled from flat sheets of a light but rigid aluminium clad foam.

\footnotetext{
${ }^{23}$ NEE-001-weiss by Dr. Neumann Peltier Technik, Utting, Germany; for a characterisation of this glue, see [58].

${ }^{24}$ EpoTek 129-4, by Epoxy Technology, Billerica, Massachusetts, USA.
} 


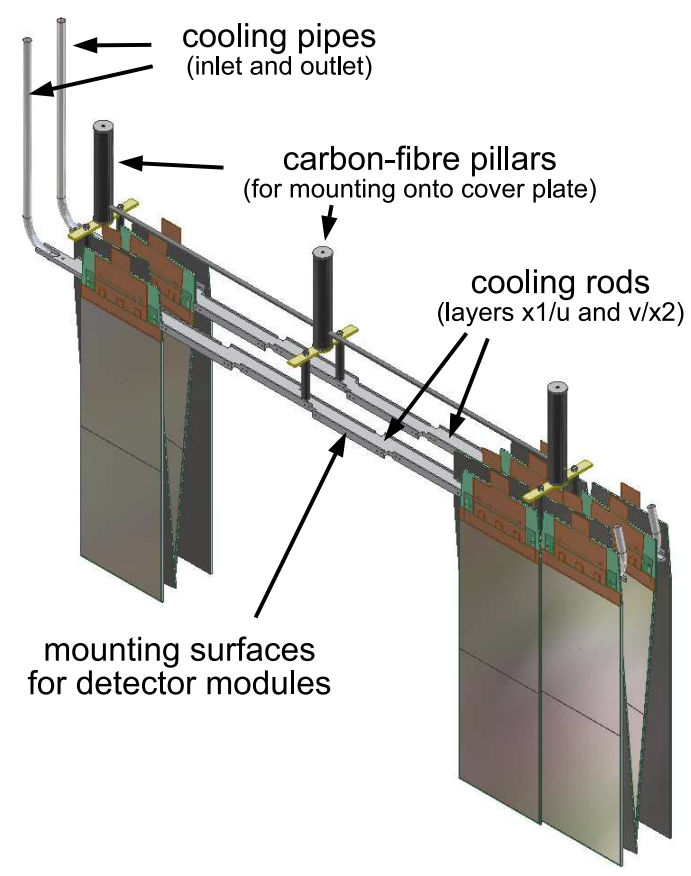

Figure 5.27: View of an IT cooling rod with a few detector modules.

Cover plate. The cover plate is made from a a $14 \mathrm{~mm}$ thick polymethacrylimide foam (Rohacell) sandwiched in between two layers of carbon fibre composite. Four printed-circuit boards, one for each detection layer, are inserted vertically through slits in the cover plate. They have four copper layers and serve to feed supply voltage and detector and control signals through the box. The outer layers carry the detector bias voltage and the analog and digital supply voltage, respectively. The two inners layers are used for the differential signals. Inside the detector box, the printed-circuit boards carry 60-pin board-to-board connectors into which the Kapton tails of the readout hybrids are plugged. Outside the box they are equipped with low mass connectors for signal cables and bias voltage cables. Cooling and nitrogen supply pipes also pass through the cover plate. Finally, the cover plate contains mounting holes for fixing the detector box on the IT support frames described below.

Cooling rods. An isometric view of the two cooling rods is shown in figure 5.27. They are mounted on the cover plate using pillars made out of a carbon fibre composite. Each cooling rod is machined out of a single piece of aluminium. It consists of a $3 \mathrm{~mm}$ thick central part and vertical mounting surfaces for each of the detector modules. An aluminium cooling pipe with an outer diameter of $6 \mathrm{~mm}$ and a wall thickness of $0.4 \mathrm{~mm}$ is glued into a semicircular groove that runs along the central part of the cooling rod. The mounting surfaces for the detector modules are $6 \mathrm{~mm}$ high and $70 \mathrm{~mm}$ wide and contain precision holes and pins to ensure the accurate positioning of the modules. Detector modules are mounted on both sides of the cooling rod, such that each cooling rod supports a pair of detection layers. The cooling pipes on the two cooling rods are connected in series using a short nitrile rubber hose.

Box enclosure. The box enclosure is assembled from $6 \mathrm{~mm}$ thick, flat sheets of polyisocyanurat (PIR) foam reinforced with a single, $200 \mu \mathrm{m}$ thick carbon fibre skin and clad on both sides 


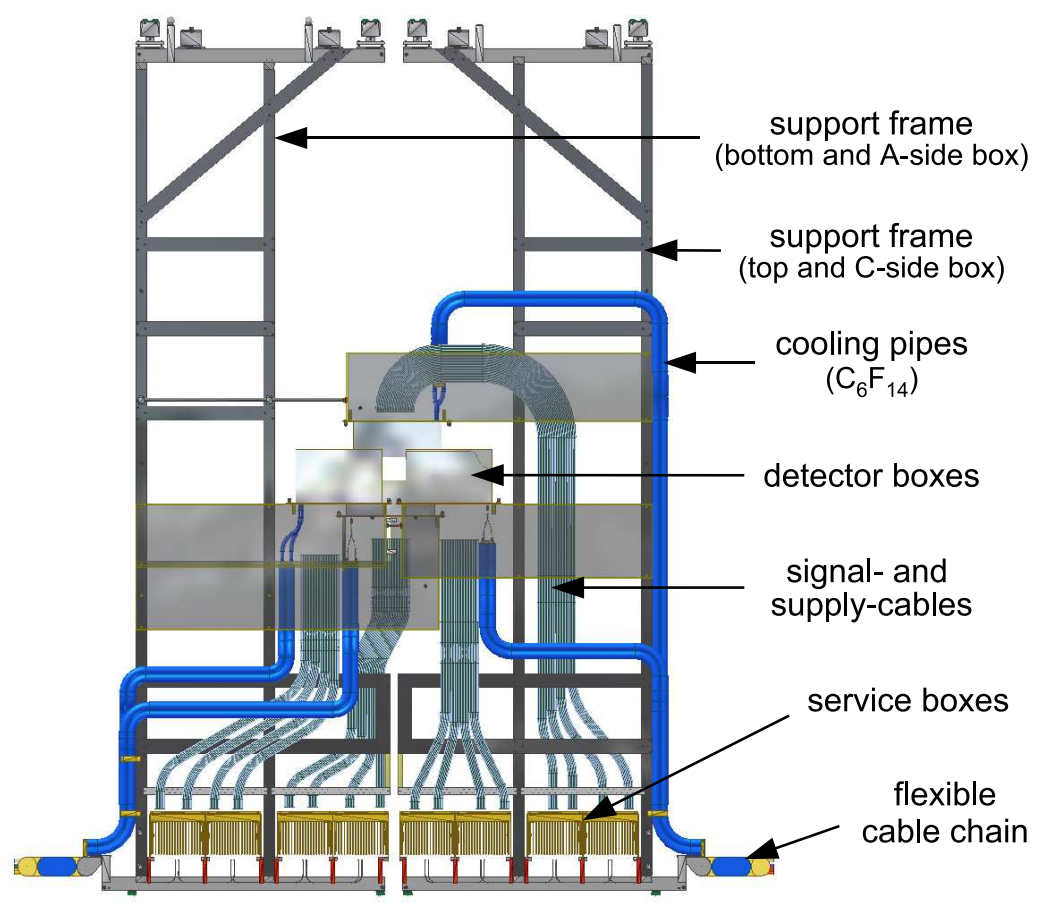

Figure 5.28: Front view of an IT detector station.

with $25 \mu \mathrm{m}$ thick aluminium foil. For the side of the box facing the beampipe, the wall thickness is reduced to $2 \mathrm{~mm}$ to decrease the distance between the beam and the innermost detector modules. Mounted on the inside wall of the enclosure is a distribution channel for the nitrogen with which the box is flushed. Small inserts made of fibreglass reinforced epoxy are embedded in the upper rim of the enclosure and permit to screw it onto the cover plate.

\section{IT detector station}

A front view of a detector station is shown in figure 5.28.

The detector boxes are mounted onto two support frames that are mounted on rails and can be retracted horizontally for detector maintenance and bakeouts of the LHC beampipe. The support frames are suspended from the upper support rail, which is mounted onto the Outer Tracker bridge (see section 5.3) and are guided by the lower rail that is mounted onto the LHCb bunker. The innermost sections of both support rails are precision machined to ensure an accurate positioning of the support frames in data taking position. The support frames are assembled from rectangular rods made of fibreglass reinforced epoxy and carbon fibre composite and from flat plates of aramide honeycomb clad with skins of a carbon fibre composite. Signal and bias voltage cables and flexible supply lines for $\mathrm{C}_{6} \mathrm{~F}_{14}$ and nitrogen are routed along the support frames from the detector boxes to the lower end of the support frame. Here, the service boxes are mounted in which the detector signals are prepared for digital optical transmission to the counting house as described in section 5.2.3. Optical fibres, cables and supply tubes are further routed through a flexible cable chain that is fixed to the lower end of the support frame at one end and to the edge of the $\mathrm{LHCb}$ bunker at the other end. 


\subsubsection{Electronics}

A central position in the ST electronics is occupied by the service boxes [59] that have already been mentioned in the descriptions of the TT and IT detector stations. The service boxes are located close to the detectors but outside of the acceptance of the experiment. They are custommade crates that hold 12 (TT) or 16 (IT) digitizer cards, a backplane for the distribution of control signals and low voltage, and a control card that provides interfaces to the LHCb Timing and Fast Control system (TFC, see section 8.3) and Experiment Control System (ECS, see section 8.4). On the digitizer cards, the analog output signals from the front-end readout chips are digitized, multiplexed and converted to optical signals. They are further transmitted via $120 \mathrm{~m}$ long optical fibres to the counting house, where they are received on the TELL1 board (see section 8.2).

Bias voltage for the silicon sensors and operation voltage for the readout electronics are provided by commercial voltage supplies. The grounding scheme follows LHCb grounding rules layed out in [60]. Temperature and humidity sensors are installed at various locations inside the detector boxes and on the service box backplane to monitor environmental conditions.

\section{Readout and data transmission}

Front-end chips. Both the TT and the IT make use of the Beetle front-end readout chip. ${ }^{25}$ Four (TT) or three (IT) Beetle chips are located on a front-end readout hybrid. Each Beetle chip amplifies and shapes the detector signals of 128 readout strips, samples them at the LHC bunch crossing frequency of $40 \mathrm{MHz}$, stores the sampled data in an analog pipeline, and upon a Level-0 trigger accept transmits the analog data 32-fold multiplexed via four differential output ports.

Copper cables. All output signals from one front-end hybrid are transmitted from the detector boxes to the service boxes via a shielded 68-wire twisted-pair cable. Only 32 (TT) or 24 (IT) of the 68 wires are needed for the transmission of the detector signals. The remaining wires are used to provide timing and slow-control signals, low voltage and ground from the service box to the front-end hybrid. The twisted-pair cables are between $2.7 \mathrm{~m}$ (IT bottom boxes) and $8 \mathrm{~m}$ (TT) long. Since, in the case of the IT, they go through the acceptance of the experiment, custom-made cables with significantly reduced shielding braids are employed to minimize the material budget.

Digitizer cards. Each digitizer card processes the data from one front-end hybrid. There are two variants of the card: a TT version to process the data from the four chip hybrids of the TT and an IT version to process the data from the three chip IT hybrids. The basic functional block for the processing of the signals from a single Beetle chip is illustrated in figure 5.29. Four differential line receivers convert the signals from the four Beetle output ports from differential to single-ended and match the signal levels to the input range of the ADC chips. Four single channel 8-bit ADC chips are used to digitize the data. These ADC chips operate at $40 \mathrm{MHz}$ and are phase locked to the sampling clock of the Beetle chip. The $4 \times 8$-bit wide output data from the four ADC chips is then fed into a single Gigabit Optical Link (GOL) chip [6]. The GOL multiplexes the data and encodes them to a single Gigabit Ethernet data stream with a data rate of $1.6 \mathrm{Gbit} / \mathrm{s}$. The laser driver integrated into the GOL chip is used to drive a $850 \mathrm{~nm}$ wavelength VCSEL diode that feeds the digitized optical data into a single optical fibre. This functional block is repeated four times on

\footnotetext{
${ }^{25}$ The Beetle front-end chip is a common development for ST and VELO. It has already been described in section 5.1.4.
} 


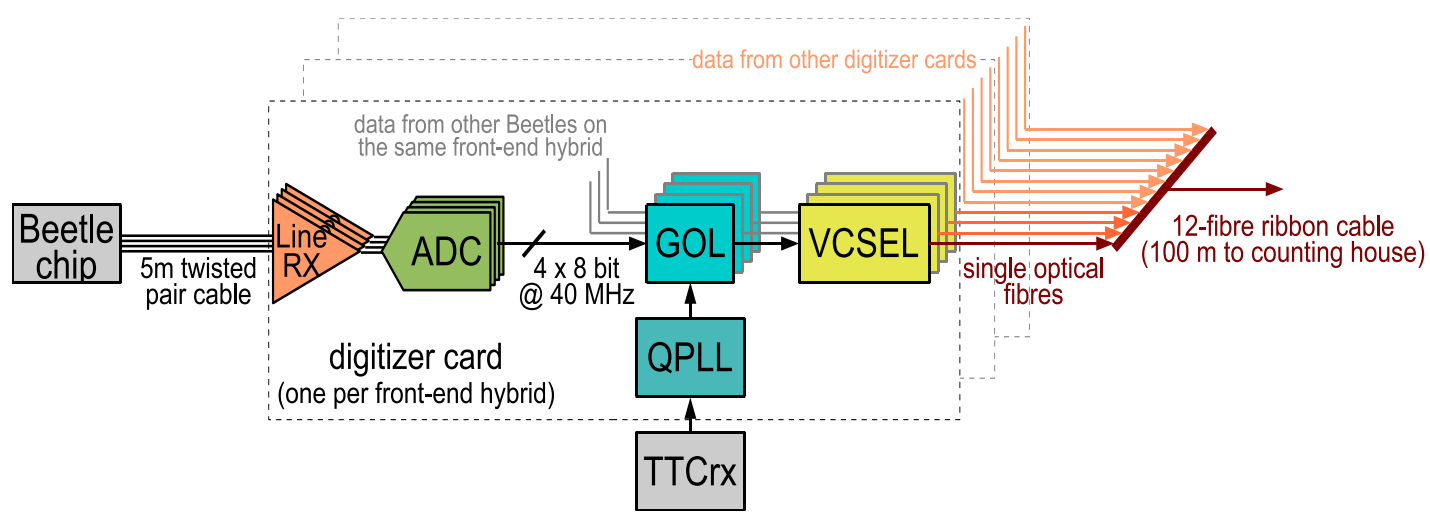

Figure 5.29: Functional block for the processing of the data from one Beetle chip. This functional block is repeated four times on a TT digitizer card and three times on an IT digitizer card.

a TT digitizer card and three times on an IT digitizer card. In addition, both variants of the card carry a central functional block for the distribution of the timing and control signals that the card receives from the service box control card.

Optical fibres. To transmit the data from the service boxes to the counting house, the outputs of twelve VCSEL diodes, corresponding to three TT digitizer cards or four IT digitizer cards, are connected to one twelve-fibre optical ribbon cable. This grouping defines a readout partitioning into groups of three readout sectors for the TT and four detector modules for the IT. The same partitioning is followed by the low voltage and bias voltage distribution and by the detector control system. A TT service box holds twelve (four groups of three), an IT service box 16 (four groups of four) digitizer cards. Both types of service boxes, therefore, feed four twelve-fibre ribbon cables.

TELL1 boards. In the counting house, two fibre ribbon cables are connected to each TELL1 board. One TELL1 board therefore receives the data from six TT readout sectors or eight IT modules. A total of 84 TELL1 boards is required to read out the 280 readout sectors of the TT and the 336 detector modules of the IT. The functionality of the TELL1 board is described in section 8.2. Pedestals are calculated and subtracted for each readout channel, common-mode noise is calculated and subtracted for each event. A cluster-finding algorithm is applied and the positions and ADC values of the clusters are transmitted to the computer farm. Non-zero-suppressed data can be transmitted for monitoring and debugging purposes.

\section{Detector control and monitoring}

Detector control and monitoring is the main task of the service box control cards [61].

TFC. The control card holds a TTCrq mezzanine [62], which collects clock, trigger and timing information from the TFC network. The TFC signals are distributed to the digitizer cards via impedance controlled differential traces on the service box backplane. All GOL chips and Beetle chips associated with the same service box receive their clock signal from the same TTCrq mezzanine. The layouts of the backplane and the digitizer cards were optimized to equalize trace lengths, resulting in signal propagation time differences that do not exceed $3 \mathrm{~ns}$.

ECS. The interface to the ECS is provided by two SPECS slave mezzanines that are mounted on each control card and that provide a total of eight $\mathrm{I}^{2} \mathrm{C}$ busses. Four of these busses are used 
to control the GOL chips per group of three (TT) respectively four (IT) digitizer cards. The other four are used to control the Beetle chips per group of three respectively four readout hybrids. In addition, $36 \mathrm{I} / \mathrm{O}$ control lines permit to individually switch off the low-voltage regulators that provide the power for the readout hybrids and digitizer cards (see below).

Monitoring. The SPECS mezzanines also provide a number of ADC channels that are employed to read out temperature sensors (PT1000) and humidity sensors at various locations in the detector boxes. Additional ADC chips are located on each digitizer card. They are employed to monitor over-current conditions of the Beetle chips and to read out a PT1000 temperature sensor that is located on each readout hybrid.

\section{Power distribution and grounding scheme}

Low voltage. Low voltage levels of $2.5 \mathrm{~V}, 3.3 \mathrm{~V}$ and $5 \mathrm{~V}$ are required for the Beetle, GOL and ADC chips, the line receivers, and various LVDS drivers on the digitizer cards and the service box backplane. They are derived from voltage levels of about $5.5 \mathrm{~V}$ and $8 \mathrm{~V}$ that are generated by MARATON power supplies ${ }^{26}$ in the LHCb cavern. The MARATON supplies are connected to the service box backplanes where they drive radiation tolerant programmable linear power regulators. ${ }^{27}$ Two of these power regulators are used to provide each readout hybrid with digital and analog power. Analog and digital power for the Beetle chips are kept separate throughout the system and are connected only on the readout hybrids. Another two power regulators provide each group of three (TT) respectively four (IT) digitizer cards with the required voltage levels, following the partitioning defined by the readout. These power regulators are located on the service box backplane and control card. They are cooled using the LHCb mixed water cooling system. The regulators for each readout hybrid and for each group of three respectively four digitizer cards can be individually switched off via the ECS.

Bias voltage. Detector bias voltage is provided by commercial high-voltage supplies ${ }^{28}$ located in the counting house. It is connected to the detector boxes via $120 \mathrm{~m}$ long cables. The HV modules can provide up to $500 \mathrm{~V}$ and deliver a current of up to $10 \mathrm{~mA}$ per channel. Only the innermost readout sectors of the TT have individual HV channels to cope with the sensor leakage currents expected after several years of operation. Everywhere else, groups of three TT readout sectors and four IT detector modules are connected to one HV channel, following the same partitioning as the readout. Each readout sector and detector module, however, has a separate supply line from a HV patch panel in the counting house to the detector. The HV patch panel carries a jumper for each readout sector and detector module. This permits to manually disconnect individual readout sectors and detector modules from their HV supply.

Grounding scheme. The grounding scheme [63] of the detector boxes is illustrated in figure 5.30. The common ground for each detector box is defined by the cooling plates (TT) respectively cooling rods (IT) onto which the detector modules are mounted as described in sections 5.2.1 and 5.2.2. Thin copper wires are employed to connect ground pads on each of the Kapton flex prints

\footnotetext{
${ }^{26}$ MARATON low voltage power supply system for hazardous hostile environment, by W-IE-NE-R, Plein \& Baus GmbH, Burscheid, Germany.

${ }^{27}$ LHC4913, developed by the CERN micro-electronics group and produced by ST Microelectronics, Geneva, Switzerland.

${ }^{28}$ CAEN SY1527 crates with A1511B modules, by CAEN S.p.A., Viareggio, Italy.
} 


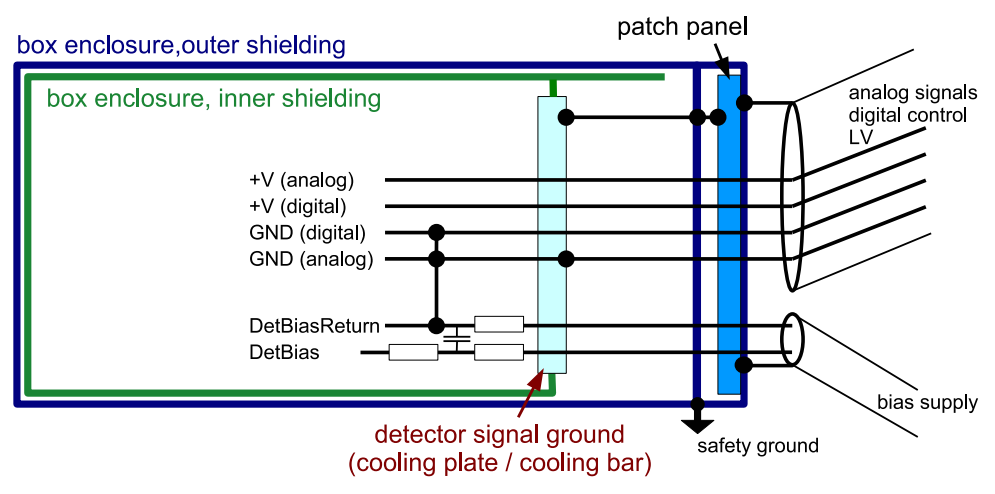

Figure 5.30: Grounding scheme of the detector boxes.

to the metallic screws with which the modules are fixed to the cooling balconies or cooling rods. The walls of the detector boxes are coated on both sides with $25 \mu \mathrm{m}$ thick foils of aluminium. The inner shielding is connected to the common ground of the detector box and the outer shielding is connected to the LHCb safety ground. The two are connected with each other at one well defined location in the detector box. The shieldings of the signal and supply cables that connect to a detector box are connected to the outer box shielding. Both the LV and HV power supplies are kept floating and are connected to the LHCb general ground only in the detector boxes. In addition, the bias voltage for each module respectively readout sector is filtered using a passive low-pass RC filter that is implemented on the front-end readout hybrids.

\subsubsection{Detector performance}

An extensive R\&D programme has been carried out to validate the detector concept, to optimize detector parameters and to estimate the expected performance of the detectors. It included simulation studies as well as various tests of prototype detectors in the laboratory and in test beams [64-68]. In view of the combination of the long readout strips and the fast pulse shapes employed, the signal-to-noise performance of the detectors was a major concern in these studies. The test beam measurements also confirmed that the expected spatial resolution of the detectors can be achieved. Other studies concerned the expected strip occupancies, which were estimated using events samples generated using the full GEANT 4 based simulation of the LHCb detector. A detailed analysis of the material budget of the detector was performed.

\section{Signal-to-noise and efficiency}

Various prototype detectors were built to establish the expected noise performance, charge collection efficiency and signal-to-noise performance of the final detectors. Effective readout strip lengths on these prototype detectors ranged from $108 \mathrm{~mm}$ up to $324 \mathrm{~mm}$. Silicon sensors were employed that measured $320 \mu \mathrm{m}$ to $500 \mu \mathrm{m}$ in thickness, had strip pitches between $183 \mu \mathrm{m}$ and $228 \mu \mathrm{m}$ and ratios of strip implant width over strip pitch from 0.25 to 0.35 . Some of the tested prototypes included Kapton interconnect cables of the same type and length as used in the M and $\mathrm{K}$ readout sectors of the TT. The performance of the prototype detectors was measured in an infrared laser 


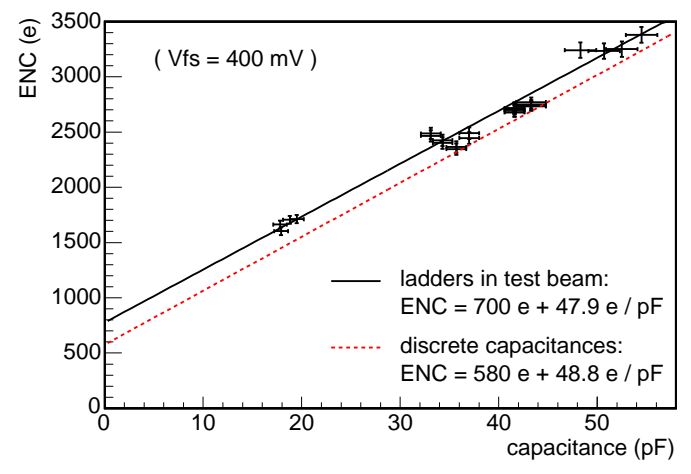

Figure 5.31: ENC obtained in test beam measurements as a function of the measured total strip capacitance of the tested prototype modules. The full line is a fit to the test beam results, the dashed line describes the results of laboratory measurements in which discrete capacitances were attached directly to the input of the Beetle chips.

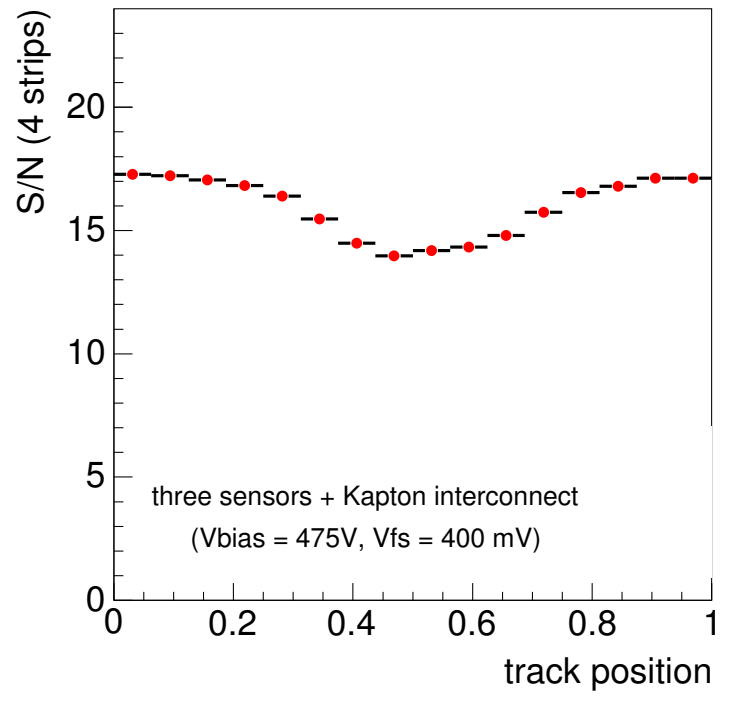

Figure 5.32: Most probable signal-to-noise ratio as a function of the inter-strip position ( 0 = centre of left strip, $1=$ centre of right strip) measured in a CERN test beam. In order to rule out a potential bias due to clustering algorithms, the signal was calculated as the sum of the charges on the four strips closest to the particle impact point given by a beam telescope.

test stand and in several test beam periods in a $120 \mathrm{GeV} \pi^{-}$beam at CERN. The expected noise performance of the various detector configurations was also investigated in a SPICE simulation that included a detailed description of the Beetle front-end, and in which the readout strips of the detectors were described as an extended LCR network [69]. The results of this simulation agreed with the test beam measurements. The measured noise performance is summarized in figure 5.31. In this figure, the measured equivalent noise charge (ENC) for the different tested detector configurations is shown as a function of their total strip capacitance. A linear dependence is observed and the slope of a line fitted to the data agrees well with that obtained in test-bench measurements of the Beetle chips, in which discrete load capacitances were attached to the Beetle inputs. Both the measurements and the SPICE simulations have demonstrated that the Kapton interconnect cables behave purely as an additional load capacitance and cause no deterioration of the quality and integrity of the detector signals.

Measurements of the charge collection efficiency were performed as a function of the position on the detector. No significant dependence was found on the position along the readout strips. However, in the direction orthogonal to the strips, a significant drop of the charge collection efficiency was observed in the central region between two readout strips. The effect is illustrated in figure 5.32 for a prototype module with the same detector geometry as a three-sensor readout sector of the TT. A similar charge loss was observed for all tested detector configurations. Its size did not depend on the strip length but was found to depend on the strip geometry. It decreased 
with increasing ratio of implant width to strip pitch $(w / p)$ and with increasing sensor thickness $(d)$ and depended roughly linearly on the ratio $(p-w) / d$. The charge loss could not be reduced by overbiasing the detectors or by increasing the shaping time within the limits allowed by the Beetle chips. It is attributed to charge trapping at the interface between the silicon bulk and the silicon oxide in between the readout strips.

This charge loss does not affect particle detection efficiency as long as the signal-to-noise ratio in the central region in between the strips remains high enough. Full particle detection efficiency above $99.8 \%$ was measured for all detector configurations as long as the most probable signal-tonoise ratio stayed above 10:1. Below that value, the particle detection efficiency started to decrease rather quickly. With the chosen thicknesses for the silicon sensors, most probable signal-to-noise ratios in excess of 12:1 are expected over the full surface of the detectors for both types of IT modules and all four types of TT readout sectors.

\section{Spatial resolution and alignment}

The spatial resolution of the detector modules was measured in test beams and was found to be about $50 \mu \mathrm{m}$, consistent with the expected resolution for the chosen strip pitches. Simulation studies have shown that, for two-strip clusters, it should be possible to further improve the spatial resolution by taking into account the position-dependent charge-sharing between the strips.

In order not to compromise the spatial resolution of the detectors, the positioning of each sensitive detector element in the $x$ coordinate should be known to better than about $25 \mu \mathrm{m}$. The relative positioning accuracy of the individual silicon sensors on a detector module was monitored throughout the module production and was found to be better than $10 \mu \mathrm{m}$ R.M.S. Various surveys of module positions inside the detector boxes and of detector boxes with respect to the LHCb reference frame have been performed. The results of these measurements are foreseen to be used to provide initial values for the software alignment of the detectors that will use reconstructed tracks from the $\mathrm{LHCb}$ spectrometer.

\section{Strip occupancies}

The strip occupancies presented here [70] were obtained from a sample of $5000 B_{d} \rightarrow J / \psi\left(\mu^{+} \mu^{-}\right)$ $K_{S}\left(\pi^{+} \pi^{-}\right)$events. The simulation software includes detailed descriptions of the detector geometry and of the signal collection, amplification and digitization. The simulation of the detector response was tuned to reproduce the results of the test beam measurements described before. For the TT, average strip occupancies of up to about $3.5 \%$ are found in the $\mathrm{K}$ sectors close to the beampipe. They drop to about $0.35 \%$ in the outermost L sectors. For the side boxes of the first IT station, average strip occupancies drop from about $2.5 \%$ on the strips closest to the beam to about $0.5 \%$ on the outermost strips. In the top/bottom boxes, occupancies vary between $0.5 \%$ and $0.3 \%$. In the second and the third IT station, occupancies are about $10 \%$, respectively $20 \%$ lower than in the first. This is mainly due to the larger distance of these stations from the beampipe supports (see section 3 ) that are located at the exit of the LHCb magnet and are a prolific source of secondary particles. 


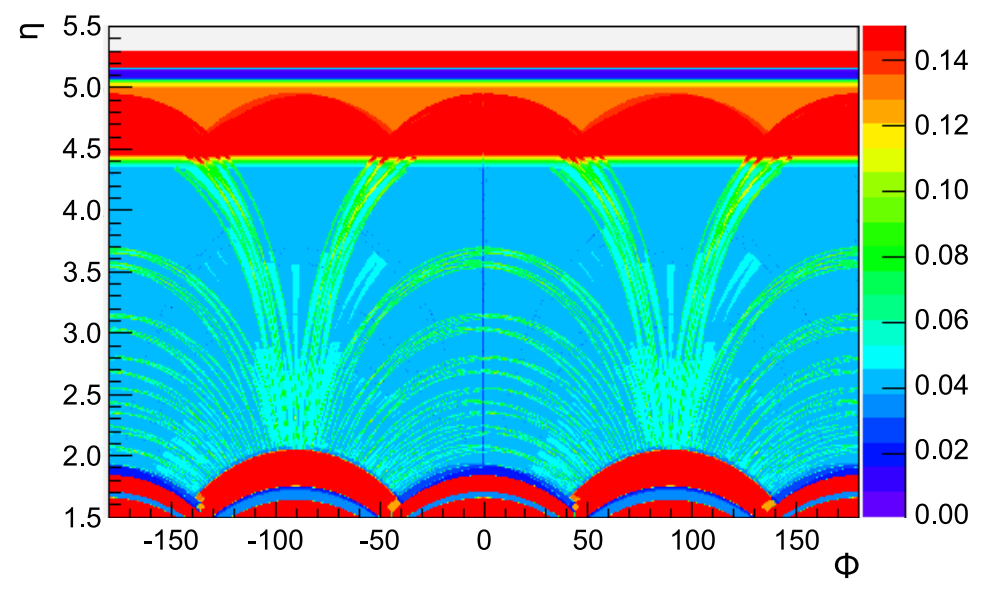

Figure 5.33: Radiation length of the TT as a function of the pseudorapidity $\eta$ and the azimuthal angle $\phi$.

\section{Material budget}

A careful analysis was performed of all materials that are located inside the acceptance of the experiment. Based on this analysis, a detailed description of all active detector elements and a simplified description of the passive components has been implemented [71, 72] in the XML based $\mathrm{LHCb}$ detector geometry description.

For the TT, where most of the dead material from detector supports, cooling etc. is located outside the acceptance, the material distribution is rather uniform. In total, it amounts to about $0.04 X_{0}$, where more than $0.02 X_{0}$ are due to the active material of the silicon sensors. An increase up to almost $0.13 X_{0}$ is observed in the very forward region, due to the material of the insulation piece around the beampipe. The result of a material scan as a function of the azimuthal angle $\phi$ and the pseudorapidity $\eta$ is shown in figure 5.33. The scan was performed by generating straight tracks originating from the nominal interaction point, extrapolating them through the TT station, and adding up the radiation lengths of all volumes in the geometry description that they crossed.

The material distribution for the IT is much less uniform, due to the readout hybrids, mechanical supports, cooling pipes and cables that are located inside the LHCb acceptance. In the active region of the detector, close to the beampipe, the material budget adds up to about $0.035 X_{0}$ per station, out of which more than $0.015 X_{0}$ are due to the active material of the silicon sensors. The material budget peaks at almost $0.30 X_{0}$ in the very narrow region of the cooling rods. The result of a material scan for one IT station is shown in figure 5.34.

\subsection{Outer Tracker}

The LHCb Outer Tracker (OT) is a drift-time detector [73], for the tracking of charged particles and the measurement of their momentum over a large acceptance area. Excellent momentum resolution is necessary for a precise determination of the invariant mass of the reconstructed b-hadrons: a mass resolution of $10 \mathrm{MeV} / \mathrm{c}^{2}$ for the decay $\mathrm{B}_{s}^{0} \rightarrow \mathrm{D}_{s}^{-} \pi^{+}$translates into a required momentum resolution of $\delta \mathrm{p} / \mathrm{p} \approx 0.4 \%$. The reconstruction of high multiplicity $\mathrm{B}$ decays demands a high tracking 


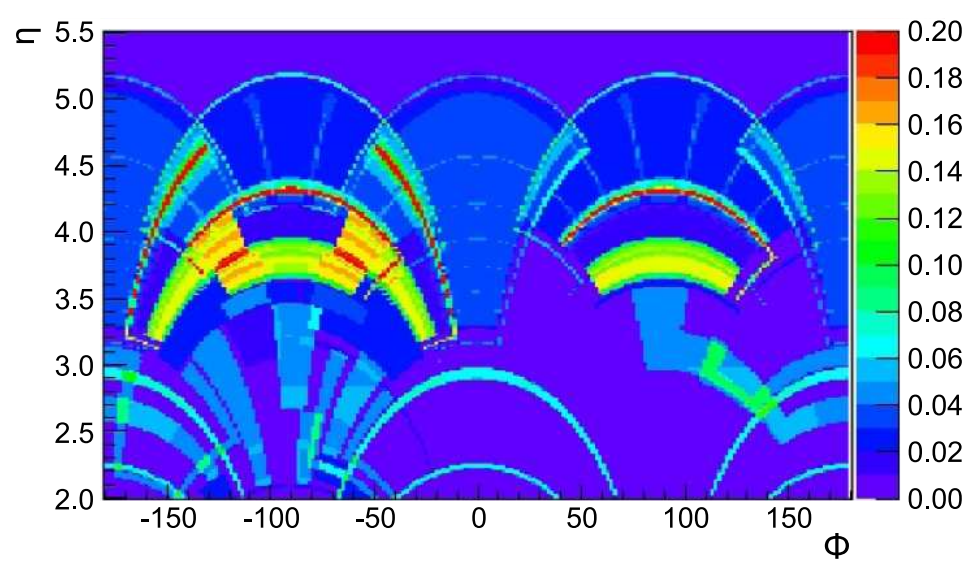

Figure 5.34: Radiation length of one IT station as a function of the pseudorapidity $\eta$ and the azimuthal angle $\phi$.

efficiency and at the same time a low fraction of wrongly reconstructed tracks: a track efficiency of $95 \%$ would result, for the decay $\mathrm{B}_{s}^{0} \rightarrow \mathrm{D}_{s}^{-} \pi^{+}$, in an overall reconstruction efficiency of $80 \%$.

\subsubsection{Detector layout}

The OT is designed as an array of individual, gas-tight straw-tube modules. Each module contains two staggered layers (monolayers) of drift-tubes with inner diameters of $4.9 \mathrm{~mm}$. As a counting gas, a mixture of Argon (70\%) and $\mathrm{CO}_{2}(30 \%)$ is chosen in order to guarantee a fast drift time (below $50 \mathrm{~ns}$ ), and a sufficient drift-coordinate resolution $(200 \mu \mathrm{m})$. The gas purification, mixing and distribution system foresees the possibility of circulating a counting gas mixture of up to three components in a closed loop [74].

The detector modules are arranged in three stations (see figure 5.35). Each station consists of four layers, arranged in an $x-u-v-x$ geometry: the modules in the $x$-layers are oriented vertically, whereas those in the $u$ and $v$ layers are tilted by $\pm 5^{\circ}$ with respect to the vertical, respectively. The total active area of a station is $5971 \times 4850 \mathrm{~mm}^{2}$. The outer boundary corresponds to an acceptance of $300 \mathrm{mrad}$ in the magnet bending plane (horizontal) and $250 \mathrm{mrad}$ in the non-bending plane (vertical). The inner cross-shaped boundary of the OT acceptance was determined by the requirement that occupancies should not exceed $10 \%$ at a luminosity of $2 \times 10^{32} \mathrm{~cm}^{-2} \mathrm{~s}^{-1}$ (this area is covered by the IT, see section 5.2.2).

The OT assembly is shown in figure 5.35. The detector modules are supported by aluminium structures. Each station is split into two halves, retractable on both sides of the beam line. Each half consists of two independently movable units of two half layers (C-frames). The modules are positionned on the $\mathrm{C}$-frames by means of precision dowel pins. The $\mathrm{C}$-frames also provide routing for all detector services (gas, low and high voltage, water cooling, data fibres, slow and fast control). The OT C-frames are sustained by a stainless steel structure (OT bridge), equipped with rails allowing the independent movement of all twelve C-frames. 

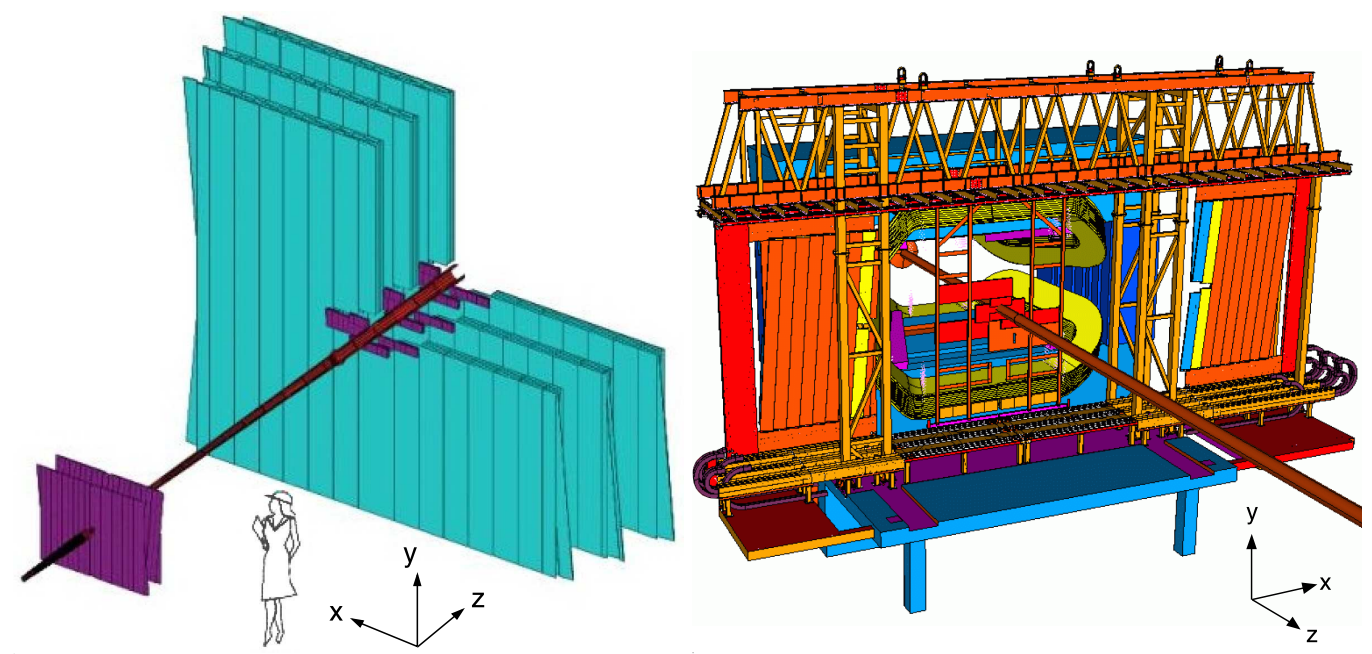

Figure 5.35: Arrangement of OT straw-tube modules in layers and stations (left) and overview of the OT bridge carrying the C-frames (right). The C-frames on both sides of the beam pipe are retracted.

\subsubsection{Detector technology}

\section{Design}

The design of the straw-tube module is based on the following requirements:

- Rigidity: the mechanical stability must guarantee the straw-tube position within a precision of 100 (500) $\mu \mathrm{m}$ in the $x(z)$ direction; the anode wire has to be centered with respect to the straw tube within $50 \mu \mathrm{m}$ over the entire straw length. The module box must be gas-tight and must withstand an overpressure of 10 mbar. The leak rate at this pressure has to be below $8 \times 10^{-4} 1 / \mathrm{s}$.

- Material budget: to limit multiple scattering and the material in front of the calorimeters, the material introduced in the OT active area must not exceed few percent of a radiation length $\mathrm{X}_{0}$ per station.

- Electrical shielding: the drift tubes must be properly shielded to avoid crosstalk and noise. Each straw must have a firm connection to the module ground. The module envelope itself must form a Faraday cage connected to the ground of the straw tubes and of the front-end electronics.

- Radiation hardness: the detector should withstand 10 years of operation at the nominal luminosity without a significant degradation of its performance. During that time the anode wires will accumulate a charge of up to $1 \mathrm{C} / \mathrm{cm}$ in the most irradiated area. As a consequence, all detector materials have to be radiation resistant and must have low outgassing.

The layout of the straw-tube modules is shown in figure 5.36. The modules are composed of two staggered layers (monolayers) of 64 drift tubes each. In the longest modules (type $F$ ) the monolayers are split longitudinally in the middle into two sections composed of individual straw 


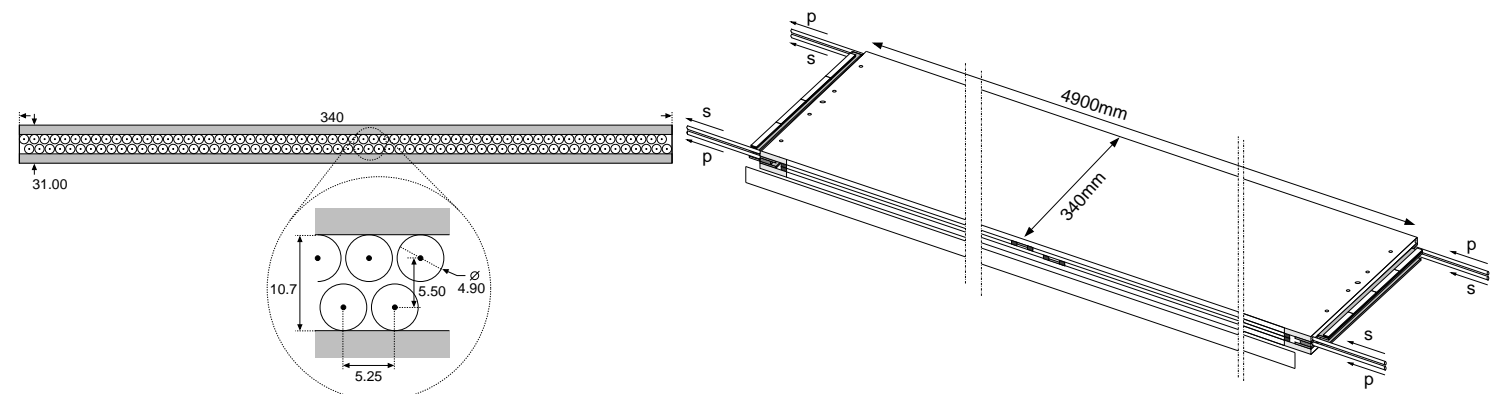

Figure 5.36: Cross section of a straw-tubes module (left) and overview of a straw-tubes module design (right).

tubes. Both sections are read out from the outer end. The splitting in two sections is done at a different position for the two monolayers to avoid insensitive regions in the middle of the module. $F$-modules have an active length of $4850 \mathrm{~mm}$ and contain a total of 256 straws. In addition to the $F$-type modules there exist short modules (type $S$ ) which are located above and below the beam pipe. These modules have about half the length of $F$-type modules, contain 128 single drift tubes, and are read out only from the outer module end. A layer half is built from 7 long and 4 short modules. The complete OT detector consists of 168 long and 96 short modules and comprises about 55000 single straw-tube channels.

\section{Construction}

The straw tubes are produced by winding together two strips of thin foils, ${ }^{29}$ as shown in figure 5.37: the inner (cathode) foil is made of $40 \mu \mathrm{m}$ carbon doped polyimide (Kapton-XC ${ }^{30}$ ); the outer foil (Kapton-aluminium) is a laminate ${ }^{31}$ made of $25 \mu \mathrm{m}$ polyimide, to enhance the straws gas tightness, and $12.5 \mu \mathrm{m}$ aluminium, crucial to ensure fast signal transmission and good shielding.

To build a monolayer the straw-tubes were glued to panels with a cored sandwich structure consisting of a $10 \mathrm{~mm}$ Rohacell core and two $120 \mu \mathrm{m}$ carbon fibre skins. High precision aluminium templates (figure 5.37) were used during the glueing to position the straw-tubes to better than $50 \mu \mathrm{m}$ over the entire module length. After the straw-tubes were glued to the panel the wiring was started. A gold-plated tungsten wire ${ }^{32}$ with a diameter of $25.4 \mu \mathrm{m}$ is used for the anodes. The wire was sucked through the straw-tube. At each end the wire is guided using injection-molded Noryl endpieces. To centre the wire also along the straw-tube Noryl wire locators had been placed every $80 \mathrm{~cm}$ inside the straws. The wires were strung with a tension of $0.7 \mathrm{~N}$ and were soldered to $5 \mathrm{~mm}$ long pads of a printed circuit board.

Special holding-devices, shown in figure 5.38, were used to keep the support panels flat to within $100 \mu \mathrm{m}$ during the glueing of the straws and wiring. They were also used to assemble two monolayer panels into a detector module (figure 5.38). The sides of the modules were closed by $400 \mu \mathrm{m}$ thick carbon fibre sidewalls. Spacers at the two module ends ensure the proper separation

\footnotetext{
${ }^{29}$ Lamina Dieletrics Ltd., UK.

${ }^{30}$ DuPont $^{\mathrm{TM}}$.

${ }^{31}$ GTS Flexible Materials Ltd., USA.

${ }^{32}$ California Fine Wire, USA.
} 


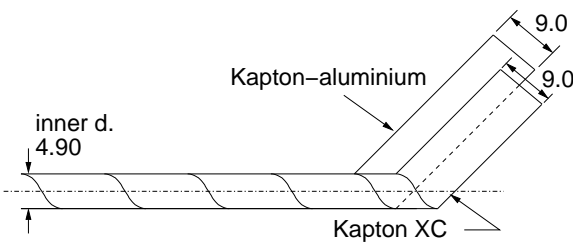

(a)

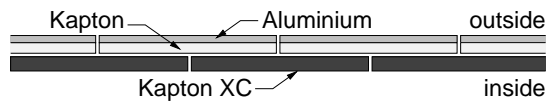

(b)

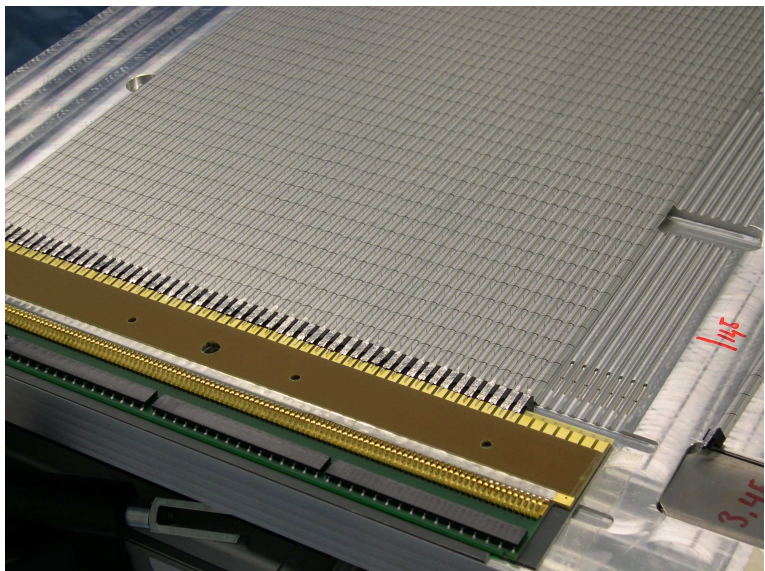

Figure 5.37: Left: (a) scheme of the straw winding using two foils; (b) Kapton-XC as inner foil and a Kapton-aluminium laminate as outer foil. Right: straws on the high precision aluminium template.
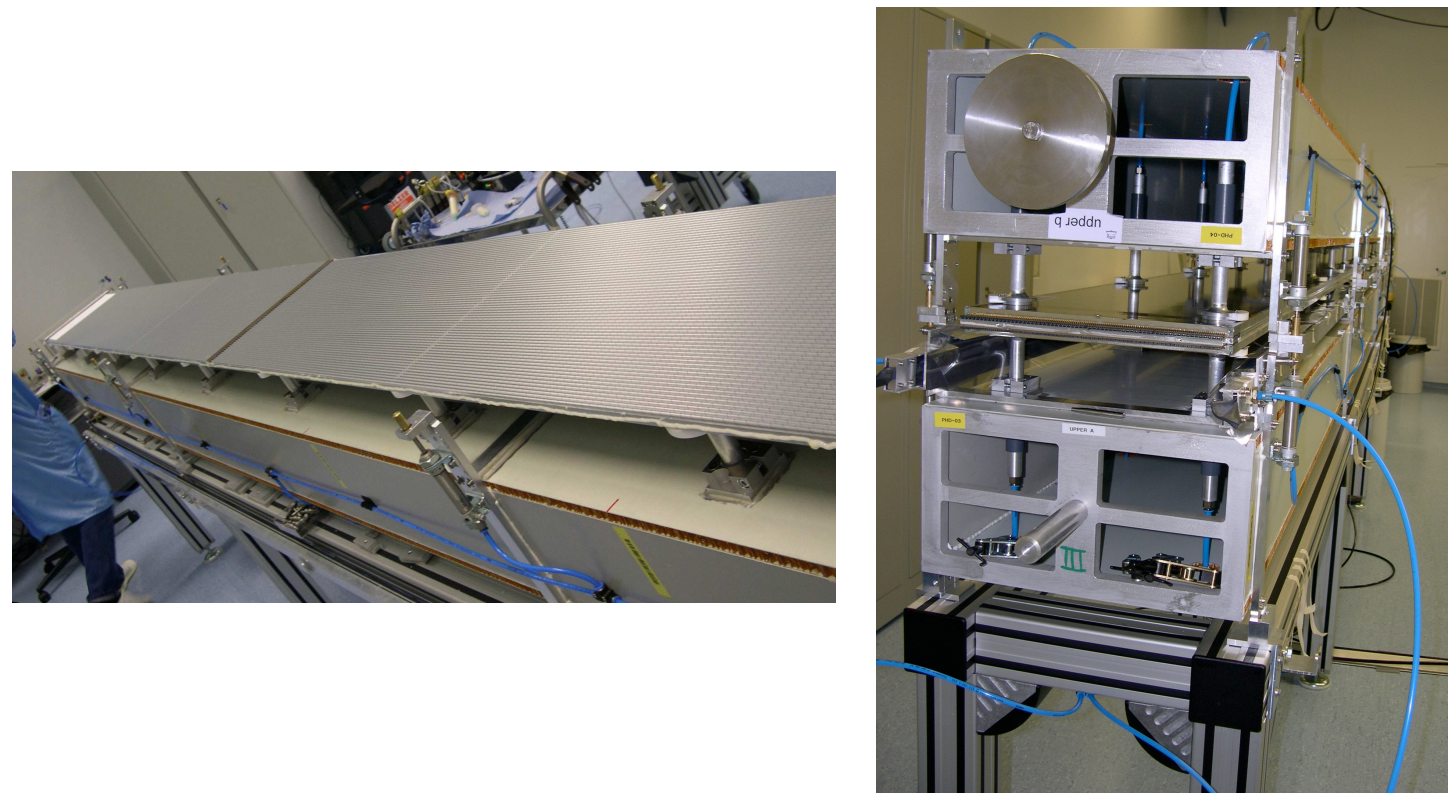

Figure 5.38: Left: straw-tube monolayer on a holding-device ready for gluing. Right: two strawtube monolayers in a holding device while being glued to form a detector module.

of the two monolayers and provide an interface to the module gas pipes. All glueing steps were performed using Araldite AY103 in combination with the HY991 hardener. The glue viscosity was enhanced by adding silica gel.

The side walls and also the inner sides of the support panels are covered by the Kapton-aluminium laminate, which ensures the gas tightness of the module and provides a closed Faraday cage.

The construction of the detector modules was completed by three production sites in about two years. All detector parts were centrally checked and then distributed to the production sites. The production procedure and the quality monitoring steps and tools were the same in all produc- 
tion sites. Quality assurance included the check of the wire tension, pitch, and leakage current (in air) prior to the module sealing. Finished modules were tested for gas tightness. Using $\mathrm{CO}_{2}$ as counting gas the modules were operated with a slowly increasing anode voltage to ensure that the leakage current per wire dropped below $1 \mathrm{nA}$ for a voltage of $1700 \mathrm{~V}$. Finally, the uniformity of the signal response of all drift cells was checked with a radioactive source.

\section{Material budget}

The forward geometry of the LHCb detector allows all detector services and supports of the OT to be located outside the detector acceptance. The OT material inside the LHCb acceptance (12 layers of modules) is estimated from the weights of the single components and the total amount of glue used (140 g per panel, $400 \mathrm{~g}$ to glue straw-tubes and to close the module) [75]. The total material of one station (four layers of modules, i.e. 8 monolayer panels) is on average equivalent to $3.2 \%$ of $\mathrm{X}_{0}$. The total OT material sums up to $9.6 \%$ of $\mathrm{X}_{0}$.

\section{Ageing tests}

In the region closest to the beam axis, the OT detector modules are foreseen to operate under particle rates per straw length of up $100 \mathrm{kHz} / \mathrm{cm}$. Extensive ageing studies of test modules and of scaled-down prototypes showed that the detector technology is resistant to radiation doses corresponding to accumulated charges of up to $1.3 \mathrm{C} / \mathrm{cm}$. No hints of a change of the gas amplificaton were seen in these studies which were performed with an average acceleration factor of a approximately 25 .

However, irradiating mass production modules with low intensity $\beta$ or $\gamma$ sources with acceleration factors of $\mathrm{O}(1)$ produced a significant drop of the gas amplification. Most strikingly, this gain loss was not observed in the region of highest irradiation intensity (anode currents around 20 $\mathrm{nA} / \mathrm{cm}$ ) but at modest intensities and anode currents of $2-5 \mathrm{nA} / \mathrm{cm}$ [76]. The damage was only observed upstream (with respect to the gas flow) of the irradiating source. An analysis of the affected anode wires revealed a thin (less than $1 \mu \mathrm{m}$ thick) insulating deposit. Although the exact mechanism is not yet understood, the glue (Araldite AY103) used to build the modules is identified as cause of the depositions. A number of preventive and remedial actions have been studied: longterm flushing of the detector modules with gas $\left(\mathrm{CO}_{2}\right)$ as well as warming-up the modules to $40^{\circ} \mathrm{C}$ while being flushed, significantly reduce this effect. Large anode currents deliberately provoked by increasing the high voltage above the nominal operating voltage can be used to clean the anode wires, should the insulating deposits reduce the gain beyond an acceptable level.

\subsubsection{Electronics}

The front-end (FE) electronics measures the drift times of the ionization clusters produced by charged particles traversing the straw-tubes with respect to the beam crossing (BX) signal [77]. The drift times are digitized for every bunch crossing ( $25 \mathrm{~ns})$ and stored in a digital pipeline to await the Level-0 decision. On a positive L0 decision, the digitized data of up to 3 bunch crossings (to cover a time range of up to $75 \mathrm{~ns}$ ) is transmitted via optical links to the Level-1 buffer (TELL1) 

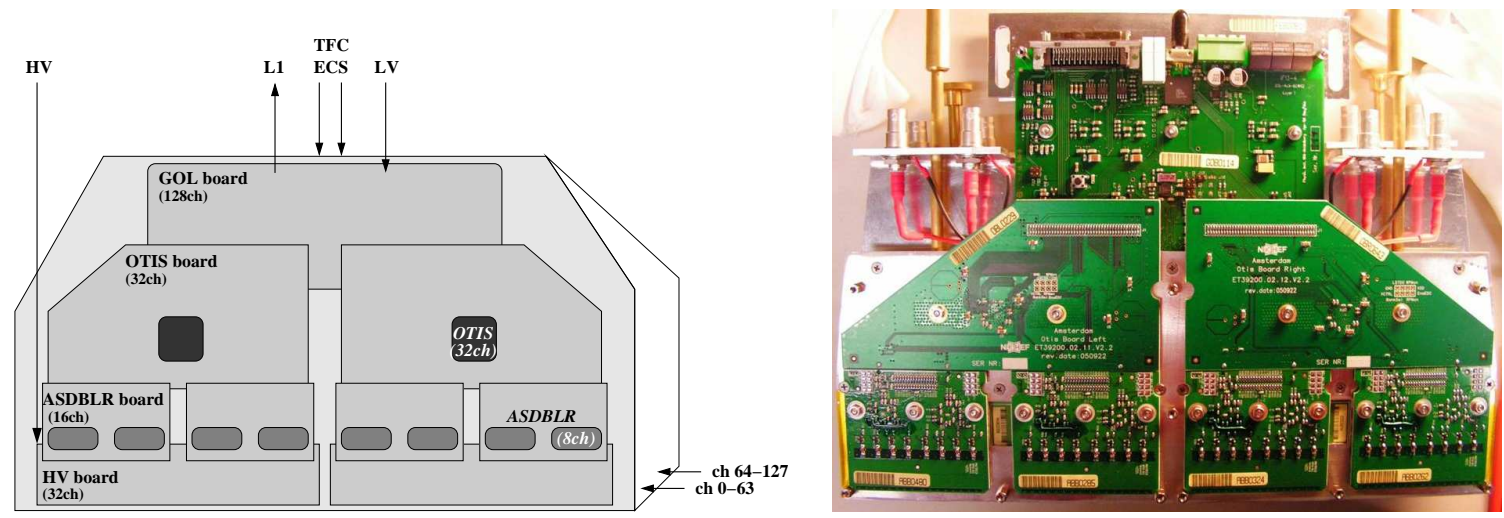

Figure 5.39: Design (left) and photograph (right) of the FE electronics mounted in a FE box. In the photograph the HV boards are not visible, because hidden by the ASDBLR boards.

boards. The radiation dose expected for the front-end electronics is only $10 \mathrm{kRad}$, well below the maximum tolerable dose of 2 Mrad.

As shown in figure 5.39, the FE electronics has a modular design, consisting of several interconnected boards housed inside a metallic box (FE box). These boxes are mounted at each end of the detector modules. A FE box is the smallest independent readout unit of the OT: the digitized data of the 128 channels of one module are sent via an optical link and received by the TELL1 board; high- and low-voltage, as well as fast- and slow-control signals are connected to each FE box individually. In total, $432 \mathrm{FE}$ boxes are used to read out the OT detector.

The main components of the OT readout electronics are the High Voltage (HV) board, the ASDBLR board, the OTIS board, and the GOL auxiliary (GOL/AUX) board.

Four 32-channel HV boards plug directly into the signal feedthrough of each straw-tube module. The signal feedthrough is provided by a passive printed circuit board (PCB) built into the module end and defining the reference ground for the straw-tubes and readout electronics. The HV board has a single HV connection and is thus the smallest independent HV supply unit. Anode signals are decoupled using $300 \mathrm{pF}$ capacitors ${ }^{33}$ on the PCB.

Each ASDBLR board hosts two ASDBLR chips [78]. These are custom integrated circuits, providing the complete analog signal processing chain (amplification, shaping, baseline restoration, and discrimination) for straw sensors. The ASDBLR is implemented in bipolar technology and produced with the radiation hard DMILL process It includes eight identical channels per chip, with a peaking time of 7-8 ns, selectable shaping circuits and a three-state LVDS output. The Equivalent Noise Charge (ENC) at the ASDBLR input is about $2200 \mathrm{e}^{-}+140 \mathrm{e}^{-} / \mathrm{pF}$, corresponding to a global $\mathrm{ENC}$ of about $0.9 \mathrm{fC}$ when connected to the detector.

The hit outputs of two ASDBLR boards (32 channels) are connected to an OTIS board, which hosts one OTIS chip for drift time digitization. The OTIS ASIC is a radiation hard 32-channel Time-to-Digital Converter (TDC), developed specifically for the OT and manufactured in a standard $0.25 \mu \mathrm{m}$ CMOS process $[79,80]$. The OTIS block diagram is shown in figure 5.40. The TDC core measures the arrival time of the ASDBLR signals with respect to the LHC clock propagating through a $25 \mathrm{~ns}$ long Delay Locked Loop (DLL), a regulated chain of 32 double-staged

${ }^{33}$ JOHANSON 302R29W331KV4E. 


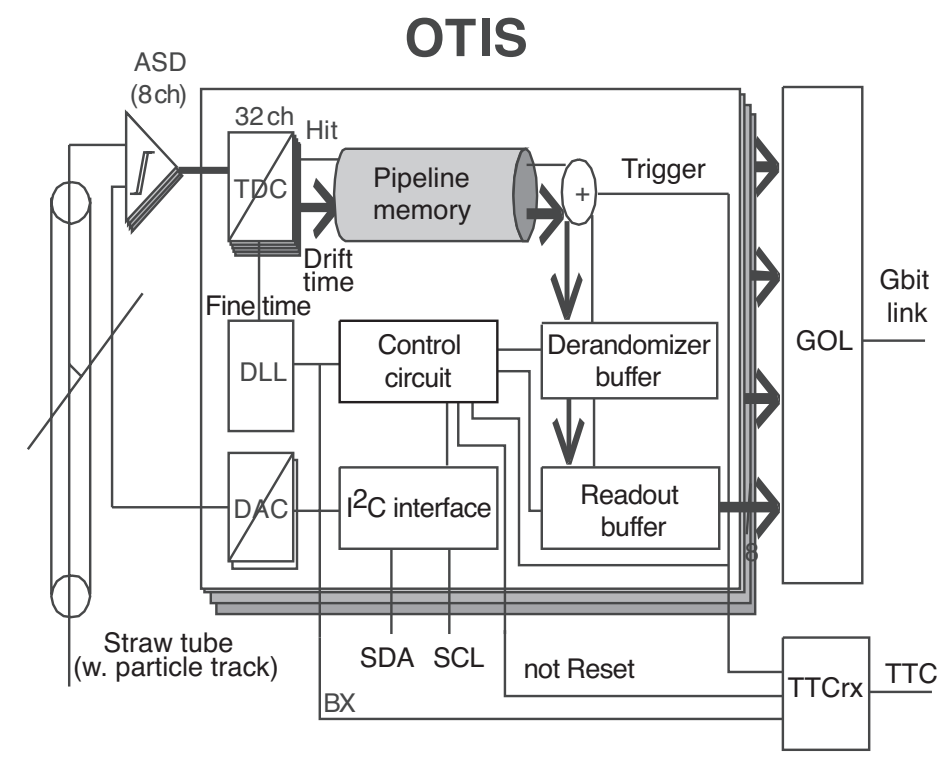

Figure 5.40: OTIS Block Diagram.

delay elements. The time digitization is done using the 64 delay-stages of the DLL (64 time bins) giving a theoretical bin size of $390 \mathrm{ps.} \mathrm{The} \mathrm{drift} \mathrm{time} \mathrm{data} \mathrm{is} \mathrm{stored} \mathrm{in} \mathrm{a} \mathrm{pipeline} \mathrm{memory} \mathrm{with} \mathrm{a}$ depth of 164 events, allowing a L0 trigger latency of 160 clock cycles to compensate for trigger rate fluctuations. If a trigger occurs, the corresponding data words of up to 3 bunch crossings (the number of bunch crossings transferred is selectable) are transferred to a derandomizing buffer, able to store data from up to 16 consecutive triggers. Both, pipeline memory and derandomizing buffer are dual-ported SRAM memories. A control unit processes and reads out the data of each triggered event within $900 \mathrm{~ns}$. The readout interface of the OTIS chip is 8-bit wide.

The data processing within the OTIS is clock driven. The chip operates synchronous to the $40 \mathrm{MHz}$ LHC clock. A standard $\mathrm{I}^{2} \mathrm{C}$ interface is used for setup and slow control.

The OTIS boards in a FE box are connected to one GOL/AUX board. This board [81] provides the outside connections to the FE box: the power connection, the interface to the fast-control (BX clock, triggers, resets) and the interface to the slow-control $\left(\mathrm{I}^{2} \mathrm{C}\right)$. Three power regulators supply the different voltages $(+2.5 \mathrm{~V} \pm 3.0 \mathrm{~V})$ needed by the OTIS and the ASDBLR chips. The GOL/AUX board hosts the GOL optical serializer chip [6] connected to an optical receiver mezzanine card on the TELL1 board.

As the data volume of the OT is large compared to other sub-detectors only the optical links of the nine FE boxes belonging to a layer quadrant are connected to a single TELL1 board. To read out the 432 FE boxes of the OT 48 TELL1 boards are used.

\section{Cooling}

As the FE box is entirely closed, cooling of the electronic components inside the boxes is necessary. All electronic boards are therefore mounted on a cooling frame (see figure 5.39) which is screwed to water-cooled plates on the $\mathrm{C}$-frames. 

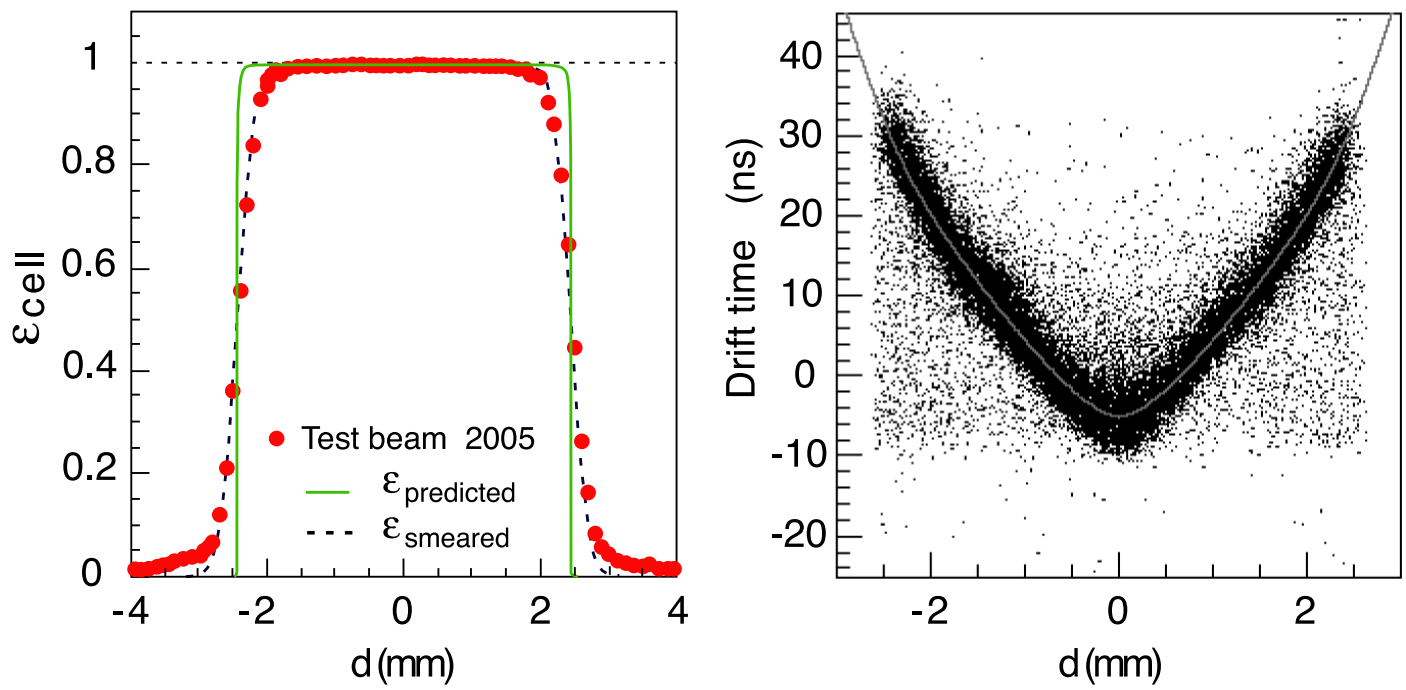

Figure 5.41: Left: hit efficiency as a function of the distance to the anode wire. Right: relation between the drift time and the calculated hit distance to the wire. Note the maximum drift time of approximately $45 \mathrm{~ns}$.

\section{High-voltage system}

Each FE box has four independent HV connections, one for each 32-channels HV board. Two CAEN SY1527LC mainframes, each equipped with four 28-channels A1833BPLC supply boards, are used as HV supply. Using an 8-to-1 distribution scheme the $1680 \mathrm{HV}$ connections of the detector are mapped on 210 CAEN HV channels. The distribution is realized using a patch panel wich offers the possibility to disconnect individual HV boards (32 channels) by means of an HV jumper. Both components, the HV supply as well as the patch panel are located in the counting house. Access to the HV system during data taking is therefore possible.

\subsubsection{Test Beam results}

To determine the performance of the detector modules in combination with the readout electronics, 4 short modules from the mass production equipped with FE boxes were tested at the $6 \mathrm{GeV}$ electron beam of the DESY II facility in Hamburg [82]. Although the number of OT modules (4 modules, or 8 monolayers) was sufficient to allow full track reconstruction, a silicon strip telescope was used to provide redundant information for the determination of the coordinates at which the beam particle traverses the detector. The trigger signal (which was also used offline as a time reference) was generated by a coincidence of two scintillator counters installed downstream of the OT modules. The nominal counting gas mixture $\mathrm{Ar} / \mathrm{CO}_{2}$ was used.

The electron beam illuminated up to 7 straws per monolayer. In the offline analysis, the relation between the measured drift time and the distance to the wire (R-t relation, see figure 5.41) was established using the predicted distance of closest approach of the particle to the anode wire. The R-t relation was then used to convert measured drift times to coordinates. The hit finding efficiency, shown as a function of the distance to the wire in figure 5.41, is determined by verifying 

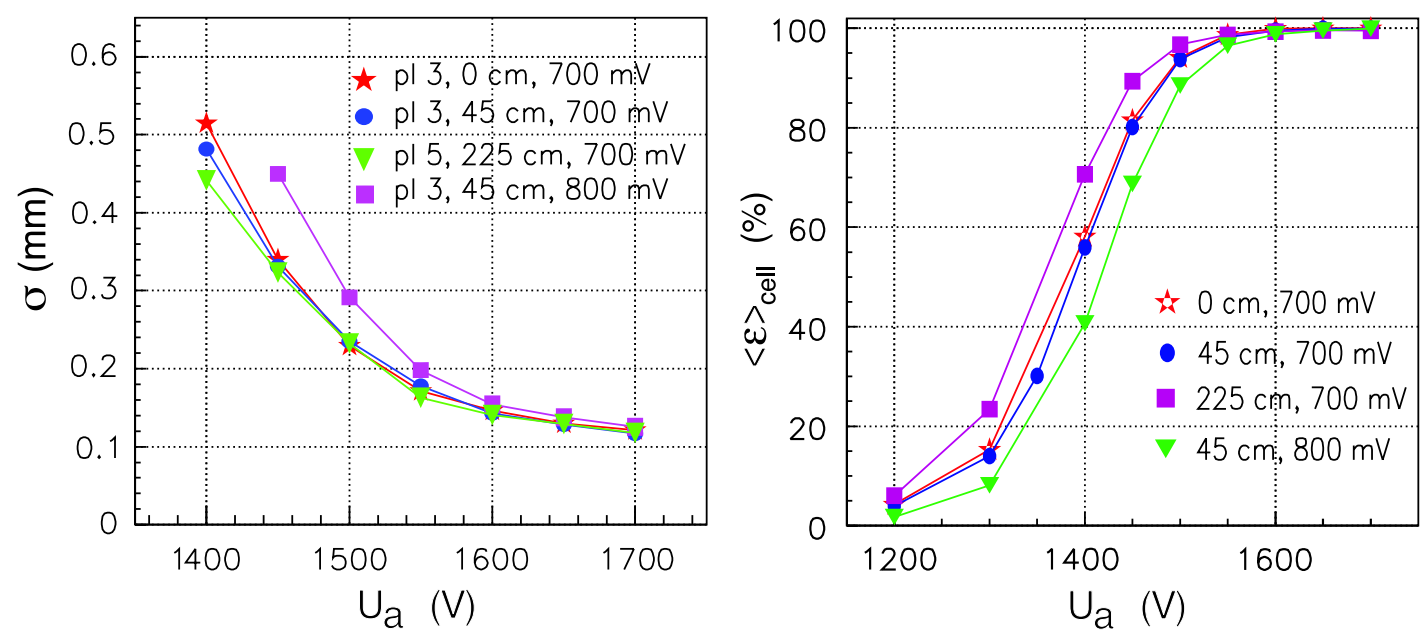

Figure 5.42: Performance of the straw-tube module: the position resolution (left) and the average efficiency (right) of a single cell are shown as a function of the HV value. The different curves correspond to different discriminator threshold voltages, and different distances between the electron beam spot and the front-end electronics.

whether the OT produced a hit at the predicted position. The position resolution is determined by comparing the measured hit coordinates to the predicted ones (residual) and by fitting a single gaussian to the distribution.

The performance of the OT straw-tube modules is determined for HV values ranging between 1200 and $1700 \mathrm{~V}$, and for ASDBLR discriminator thresholds ranging from $1.5 \mathrm{fC}$ to $5.5 \mathrm{fC}$. As shown in figure 5.42, efficiencies larger than 99\% in the centre of the straw (dropping at the edge of the straw) and position resolutions of single cells below $200 \mu \mathrm{m}$ can be attained for high voltage values above $1550 \mathrm{~V}$. The noise level was found to be low: at a high voltage of $1550 \mathrm{~V}$ and a discriminator threshold voltage of $800 \mathrm{mV}$ (corresponding to a signal threshold of $4 \mathrm{fC}$ ), the noise rate is below $1 \mathrm{kHz}$ per channel. This corresponds to an average channel occupancy of $\left(7.5 \times 10^{-3}\right) \%$. The probability to find a coherent hit in neighbouring channels (crosstalk) is found to be lower than $5 \%$ for high voltage values below $1600 \mathrm{~V}$ and discriminator threshold voltages above $800 \mathrm{mV}$ (4 fC).

\subsubsection{Alignment and monitoring}

Errors in the mechanical alignment of the drift tubes can significantly degrade the track reconstruction. A single cell resolution of about $200 \mu \mathrm{m}$ requires that the drift tubes be aligned within an accuracy of $100 \mu \mathrm{m}(1 \mathrm{~mm})$ in the $x(z)$ coordinate. Therefore, care was taken during each step of the detector construction and installation to minimize alignment errors. The issue of the mechanical tolerances in the module production has been discussed in section 5.3.2. All detector $\mathrm{C}$-frames which hold the modules were built with stringent requirements on the mechanical tolerances [83, 84]. During installation the positions of all modules were surveyed and the C-frames positions have been adjusted accordingly.

The reproducibility of the $\mathrm{C}$-frames positioning after movement was checked to be better than the $200 \mu \mathrm{m}$ precision of the optical survey. 


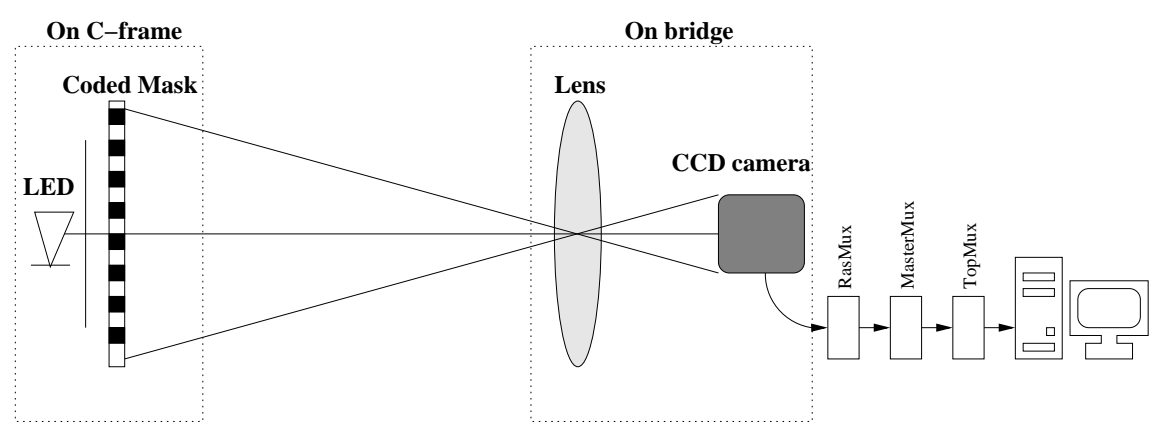

Figure 5.43: Schematic of the RASNIK alignment system.

The stability of the C-frames relative position during data taking is monitored by means of the RASNIK system [85], whose basic idea is to project the image of a detailed pattern through a lens onto a CCD camera (figure 5.43). Movements perpendicular to the optical axis are observed as change of the pattern position processed by the CCD camera, whereas movements along the axis are measured by the change in the image size. A total of 50 RASNIK lines are used for the OT monitoring. The intrinsic resolution of the system perpendicular (parallel) to the beam axis is better than 10 (150) $\mu \mathrm{m}$ respectively [86]. 


\section{Chapter 6}

\section{Particle identification}

\subsection{RICH}

Particle identification (PID) is a fundamental requirement for LHCb. It is essential for the goals of the experiment to separate pions from kaons in selected B hadron decays. At large polar angles the momentum spectrum is softer while at small polar angles the momentum spectrum is harder; hence the particle identification system consistes of two RICH detectors to cover the full momentum range. The upstream detector, RICH 1 , covers the low momentum charged particle range $\sim 1-$ $60 \mathrm{GeV} / \mathrm{c}$ using aerogel and $\mathrm{C}_{4} \mathrm{~F}_{10}$ radiators, while the downstream detector, RICH 2, covers the high momentum range from $\sim 15 \mathrm{GeV} / \mathrm{c}$ up to and beyond $100 \mathrm{GeV} / \mathrm{c}$ using a $\mathrm{CF}_{4}$ radiator (see figure 6.1). RICH 1 has a wide acceptance covering the full $\mathrm{LHCb}$ acceptance from $\pm 25 \mathrm{mrad}$ to $\pm 300 \mathrm{mrad}$ (horizontal) and $\pm 250 \mathrm{mrad}$ (vertical) and is located upstream of the magnet to detect the low momentum particles. RICH 2 is located downstream of the magnet and has a limited angular acceptance of $\sim \pm 15 \mathrm{mrad}$ to $\pm 120 \mathrm{mrad}$ (horizontal) and $\pm 100 \mathrm{mrad}$ (vertical) but covers the region where high momentum particles are produced. In both RICH detectors the focusing of the Cherenkov light is accomplished using a combination of spherical and flat mirrors to reflect the image out of the spectrometer acceptance. In the RICH 1 the optical layout is vertical whereas in RICH 2 is horizontal. Hybrid Photon Detectors (HPDs) are used to detect the Cherenkov photons in the wavelength range $200-600 \mathrm{~nm}$. The HPDs are surrounded by external iron shields and are placed in MuMetal cylinders to permit operation in magnetic fields up to $50 \mathrm{mT}$. The RICH detector system including its electronics, monitoring, control, and the performance are described below.

\subsubsection{RICH 1}

The RICH 1 detector $[2,87]$ is located upstream of the $\mathrm{LHCb}$ dipole magnet, between the VELO and the Trigger Tracker, see figure 2.1. RICH 1 contains aerogel and fluorobutane $\left(\mathrm{C}_{4} \mathrm{~F}_{10}\right)$ gas radiators, providing PID from approximately $1--60 \mathrm{GeV} / c$ for particles inside the acceptance. A schematic, 3D model and photo of the RICH 1 detector is shown in figure 6.2. It is aligned to the $\mathrm{LHCb}$ coordinate axes and occupies the region $990<z<2165 \mathrm{~mm}$. The $z$-axis follows the beamline which is inclined at $3.6 \mathrm{mrad}$ to the horizontal. The overall design has to respect the following constraints: 


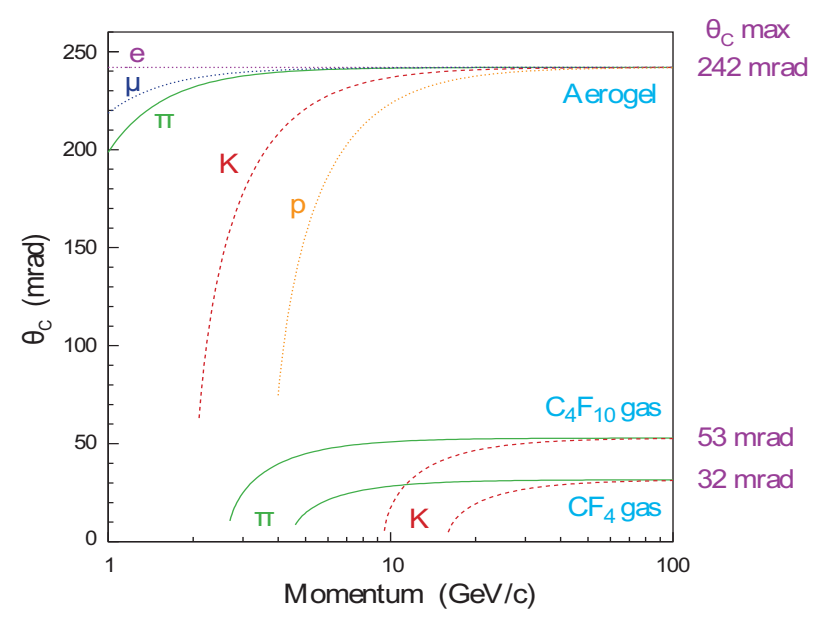

Figure 6.1: Cherenkov angle versus particle momentum for the RICH radiators.

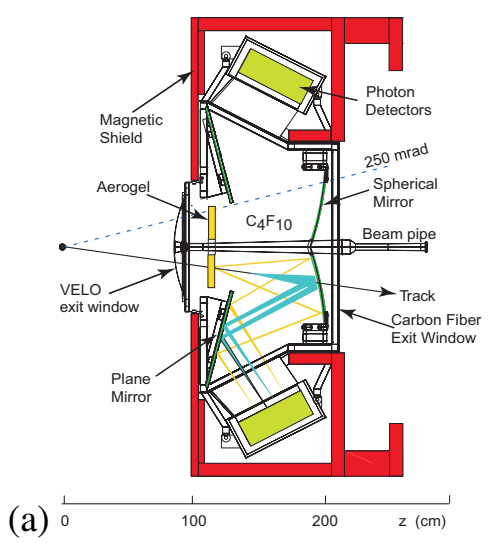

(b)
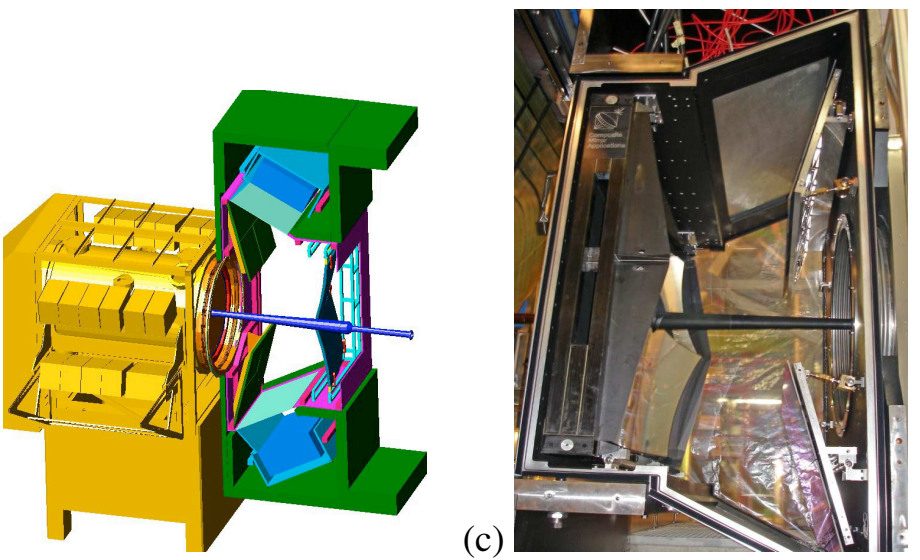

Figure 6.2: (a) Side view schematic layout of the RICH 1 detector. (b) Cut-away 3D model of the RICH 1 detector, shown attached by its gas-tight seal to the VELO tank. (c) Photo of the RICH1 gas enclosure containing the flat and spherical mirrors. Note that in (a) and (b) the interaction point is on the left, while in (c) is on the right.

- minimizing the material budget within the particle acceptance of RICH 1 calls for lightweight spherical mirrors with all other components of the optical system located outside the acceptance. The total radiation length of $\mathrm{RICH} 1$, including the radiators, is $\sim 8 \% \mathrm{X}_{0}$.

- the low angle acceptance of RICH 1 is limited by the $25 \mathrm{mrad}$ section of the LHCb beryllium beampipe (see figure 3.1) which passes through the detector. The installation of the beampipe and the provision of access for its bakeout have motivated several features of the RICH 1 design.

- the HPDs of the RICH detectors, described in section 6.1.5, need to be shielded from the fringe field of the LHCb dipole. Local shields of high-permeability alloy are not by themselves sufficient so large iron shield boxes are also used. 
The HPDs are located outside of the LHCb acceptance, above and below the beamline, in a region where the iron shield can be accommodated. Additional planar (flat) mirrors are required to reflect the image from the tilted spherical mirrors onto the photon detector planes.

\section{Optical system}

The parameters of the RICH 1 optical layout have been optimized with the aid of simulation. Charged particle tracks, originating from the interaction point (IP) are followed through the RICH 1 radiators. Cherenkov photons are generated uniformly along the length of each track in the aerogel and gas radiators, using the appropriate refractive indices for photons within the wavelength acceptance of the HPD photocathodes. These photons are ray-traced through the optical system and their impact points on the planes of HPDs are recorded. The Cherenkov angle at emission is then reconstructed for each photon in turn, assuming that the emission point is midway along the track trajectory through the radiator. As the true emission point is randomly distributed along the track, the tilted mirror geometry causes the reconstructed Cherenkov angle to differ from its true value and results in a smearing of the reconstructed angle. The RMS of the resulting distribution is referred to as the emission point error. The RICH 1 optical system is designed such that the emission point error is not larger than other sources of finite angular resolution, such as the HPD pixel size and the chromatic dispersion of the radiator.

In addition to the emission point error, the optical layout determines the required area of coverage of the two HPD planes. The optimization procedure required close to $100 \%$ geometrical acceptance for photons emitted by the gas radiator, while a compromise dictated by the cost, and not affecting significantly the final expected performance, reduced the acceptance for the aerogel photons to $68 \%$. The parameters of the optics are constrained by limited space and the tilt of the spherical mirrors must ensure that the flat mirrors lie outside the acceptance of the charged particle trackers. The location of the photon detector planes is also determined by the performance of the magnetic shield boxes. To avoid loss of efficiency, the HPD planes must be in a region where the field does not exceed $3 \mathrm{mT}$ and be tilted to ensure that, on average, Cherenkov photons strike the HPDs at close to normal incidence.

The parameters resulting from the optimization procedure have been adopted for the engineering design of RICH 1 . The spherical mirrors have radius of curvature $2700 \mathrm{~mm}$ with centres of curvature at $x, y, z=0, \pm 838,-664 \mathrm{~mm}$, which define the mirror tilt. This results in an emission point error of $0.67 \mathrm{mrad}$ for the gas radiator, a value that is negligible compared with other sources of error for the aerogel. The flat mirrors are tilted at an angle $0.250 \mathrm{rad}$ with respect to the $y$-axis, with horizontal edges lying closest to the beam line located at $y, z= \pm 350,1310 \mathrm{~mm}$. The two HPD planes are centered at $x, y, z=0, \pm 1187,1502 \mathrm{~mm}$ and tilted at an angle $1.091 \mathrm{rad}$ with respect to the $y$-axis. They are each covered with 7 rows of 14 HPDs, hexagonally close packed, with centres separated by $89.5 \mathrm{~mm}$. This results in two detector planes of $1302 \mathrm{~mm} \times 555 \mathrm{~mm}$ each.

\section{Spherical mirrors}

The spherical mirrors are located within the LHCb acceptance and are traversed by charged particles and photons. Glass mirrors and their associated supports would contribute about $8 \% \mathrm{X}_{0}$, so a carbon fibre reinforced polymer (CFRP) substrate, with the mirror support outside the acceptance, 
(a)

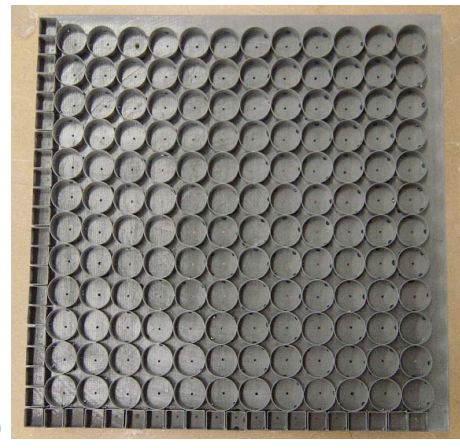

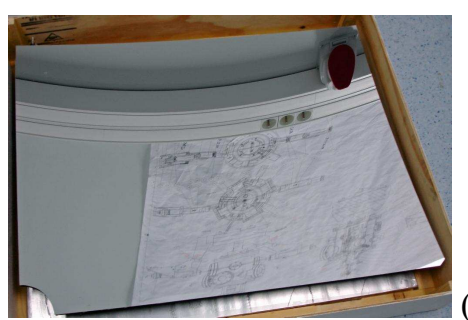

(c)

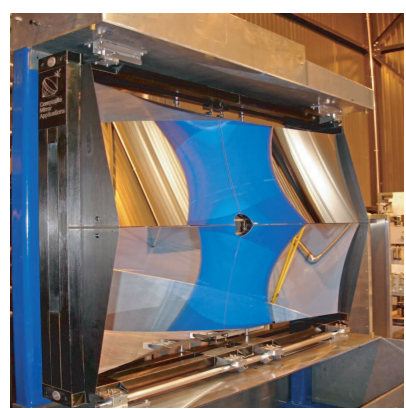

Figure 6.3: Photo of (a) the internal structure of the RICH 1 carbon fibre (CFRP) mirror; (b) a CFRP mirror; (c) the spherical mirror array viewed from the front, mounted onto its CFRP support frame.

is adopted to keep the material budget below $2 \% \mathrm{X}_{0}$. The two tilted spherical mirror surfaces, one above the beryllium beampipe, the other below, are each composed of two CFRP mirrors, making four mirror quadrants in total [88]. Each mirror has dimensions $830 \mathrm{~mm} \times 630 \mathrm{~mm}$ when projected onto the $x-y$ plane. The mirror construction is from two CFRP ${ }^{1}$ sheets, moulded over a polished glass mandrel to a spherical surface of $2700 \mathrm{~mm}$ radius and separated by a reinforcing matrix of CFRP cylinders, configured as shown in figure 6.3a. The box elements at the two outer edges provide stiffness for the three-point mounting brackets that attach the mirror to a CFRP frame (see figure 6.3c). Each mirror has a circular cut-out of radius $62.5 \mathrm{~mm}$ to provide a nominal $10 \mathrm{~mm}$ clearance from the beampipe (see figure $6.3 \mathrm{~b}$ ). The overall structure has an areal density of $6 \mathrm{~kg} \mathrm{~m}^{-2}$, thus contributing about $1.5 \% \mathrm{X}_{0}$ to the material budget of RICH 1 .

The geometrical quality of a spherical mirror is characterized by the variation in the mean radius $R$ of each mirror and the parameter $D_{0}$, the diameter of the image in which is contained 95\% of the light from a point source placed at the centre of curvature. Provided $D_{0}<2.5 \mathrm{~mm}$ and $\Delta R / R<1 \%$, the geometry of the mirrors provides a negligible contribution to the reconstructed Cherenkov angle precision. The manufacturing and subsequent quality assurance ensures that the mirrors satisfy these specifications.

The mirror assembly is made from two CFRP half frames. Each carries an upper and a lower quadrant. The half frames are divided in this way to allow insertion into the RICH 1 gas enclosure (described further on in this section) from either side of the beampipe, following which the frames are clamped to form a rigid structure around the periphery. The CFRP frame is mounted on rails and supported by an optical alignment rig. Upper and lower mirror pairs are aligned to a common centre of curvature and the CFRP frame is surveyed. This frame is then installed in the gas enclosure using an identical rail system, where the frame can again be surveyed and adjusted if necessary. Simulations [89] have shown that provided all mirror segments are aligned to a precision of about $1 \mathrm{mrad}$, the alignment can be further corrected using data from reconstructed Cherenkov ring images.

The mirrors are coated using a deposition of $\mathrm{Al}(80 \mathrm{~nm})+\mathrm{MgF}_{2}(160 \mathrm{~nm})$. The reflectivity that can be achieved on the CFRP substrate is the same as that on a glass support, as shown by curve $\mathrm{V}$ in figure 6.6 and described in section 6.1.4.

\footnotetext{
${ }^{1}$ Fibres: Toray M46J; Matrix: Bryte Technologies, EX-1515 cyanate ester resin; Manufacturer: Composite Mirror Applications Inc.,Tucson, USA.
} 


\section{Flat mirrors}

The flat mirrors are assembled into two planes, one above and one below the beamline. They are located outside of the acceptance, so glass substrates can be used. Each plane comprises eight rectangular mirrors with dimensions $380 \mathrm{~mm} \times 347.5 \mathrm{~mm}$, fabricated using $6.5 \mathrm{~mm}$ thick Simax ${ }^{2}$ glass. As for the spherical mirrors, the flat mirror geometry is characterized through the parameters $R$ and $D_{0}$. All mirrors have $|R|>600 \mathrm{~m}$ and $D_{0}<2.5 \mathrm{~mm}$. Assuming the deviations from surface sphericity are randomly distributed, this value of $D_{0}$ contributes $<0.2 \mathrm{mrad}$ to the single photon Cherenkov angle precision. The mirrors are coated using the same $\left(\mathrm{Al}+\mathrm{SiO}_{2}+\mathrm{HfO}_{2}\right)$ process used for the RICH 2 mirrors (section 6.1.2). Each of the eight mirror segments is connected to a threepoint adjustment system via a polycarbonate flange, described in section 6.1.2. The adjustment mechanism is bolted to a rigid plate that is suspended from rails. The eight mirror segments are adjusted in angle to form a single plane, then the angle of this plane is set using the optical alignment rig. Following alignment and survey, the plate is mounted on an identical rail system attached to the upstream wall of the RICH 1 gas enclosure, where the angle can again be surveyed and adjusted if necessary.

\section{RICH 1 structure}

The total weight of the RICH 1 detector is about 16 tons, mainly due to the magnetic shielding boxes. The lower box is fixed to the LHCb cavern floor and supports the gas enclosure and the lower photon detector assembly. The upper box is fixed to the cavern wall and supports the upper photon detector assembly.

\section{Gas enclosure}

The functions of the gas enclosure are to contain the $\mathrm{C}_{4} \mathrm{~F}_{10}$ gas radiator and to provide a light-tight and mechanically stable platform for all optical components. The gas enclosure must sustain a $\pm 300 \mathrm{~Pa}$ pressure differential between the $\mathrm{C}_{4} \mathrm{~F}_{10}$ gas and the outside atmospheric environment. It is essentially a six-sided box machined from $30 \mathrm{~mm}$ thick aluminium alloy tooling plate ${ }^{3}$ that is welded at the edges to form a $600 \mathrm{~kg}$ structure with a total volume of about $3.5 \mathrm{~m}^{3}$ (see figure $6.2 \mathrm{c}$ ). All six faces have apertures. The boundaries of the upstream and downstream apertures are clear of the RICH 1 acceptance region. The upstream face attaches to a $300 \mu \mathrm{m}$ thick stainless steel bellows that provides a gas-tight, mechanically compliant (axial stiffness $37 \mathrm{Nmm}^{-1}$ ) seal to the downstream face of the VELO vacuum tank. The downstream face is closed by a low-mass exit window that is sealed to a flange on the beryllium beampipe using a $1 \mathrm{~mm}$ thick opaque moulded silicone $^{4}$ diaphragm. The exit window is manufactured from a sandwich of two $0.5 \mathrm{~mm}$ thick CFRP skins separated by $16 \mathrm{~mm}$ thick polymethacrylimid foam. Its radiation length corresponds to $0.6 \% \mathrm{X}_{0}$. The choice of material thickness for the exit window is a compromise between material budget and the deflection $( \pm 4 \mathrm{~mm})$ due to the \pm 300 Pa pressure differential that will be maintained by the $\mathrm{C}_{4} \mathrm{~F}_{10}$ gas system.

\footnotetext{
${ }^{2} 3.3$ borosilicate glass by SKLÁRNY KAVALIER, a.s. COMPAS, Kinskeho 703, CZ-51101 Turnov.

${ }^{3} \mathrm{C} 250$, cast using type 5083 alloy.

${ }^{4}$ Dow Corning Sylgard 186, with 5\% black pigment added.
} 
The side faces of the gas enclosure are fully open to maximize access for installation of the optical components and to the beampipe. They are closed by $10 \mathrm{~mm}$ thick aluminium door panels. With the doors bolted in place the maximum displacement of any part (except the exit window) of the gas enclosure due to variations of gas pressure is less than $150 \mu \mathrm{m}$. Apertures above and below the beamline are sealed using windows that allow Cherenkov light to reach the HPDs. These windows are $8 \mathrm{~mm}$ thick quartz ${ }^{5}$ with dimensions $1360 \mathrm{~mm} \times 574 \mathrm{~mm}$, fabricated from two equalsize panes, glued together along one edge. The quartz windows are coated with $\mathrm{MgF}_{2}$ to reduce surface reflection losses from $8 \%$ to $4 \%$.

\section{Magnetic shield boxes}

The HPDs are located in the upstream fringe field of the LHCb dipole of about $60 \mathrm{mT}$. They operate at full efficiency at a B-field up to a maximum of $3 \mathrm{mT}$. So the shield boxes need to attenuate the external field by a factor of at least 20, they need to be large enough to accommodate the HPDs and their associated readout, and they must not obstruct Cherenkov light falling on the HPD photocathodes. In addition, the bending of charged particles in the region between the VELO and the TT station provides a momentum measurement that is important for the trigger. Therefore, in designing the magnetic shields, due consideration was given to maintaining the field integral in this region.

A schematic of the shielding structure is shown in figure 6.2. The magnetic design was optimized using the OPERA/TOSCA ${ }^{6}$ finite element modelling software. It is assembled from 50 and $100 \mathrm{~mm}$ thick high purity $\mathrm{ARMCO}^{7}$ plates. Measurements made with the shields in place and the LHCb dipole at full field indicate that the maximum B-field at the HPD plane is $2.4 \mathrm{mT}$, while the field integral between the IP $(z=0)$ and the TT $(z=2500 \mathrm{~mm})$ is $0.12 \mathrm{Tm}$. Further details of the modelling and measurements are reported in reference [90]. The overall dimensions $(x, y, z)$ of the shield are $1950 \mathrm{~mm} \times 4000 \mathrm{~mm} \times 1175 \mathrm{~mm}$. The weight of each box is about $75 \mathrm{kN}$ and the magnetic forces at full field are about $50 \mathrm{kN}$. The rigidity of the shielding structure and mounting ensures that any displacement of the HPD assembly is less than $0.5 \mathrm{~mm}$ when the LHCb magnet current is switched on.

\subsubsection{RICH 2}

The RICH 2 detector $[91,92]$ is located between the last tracking station and the first muon station, see figure 2.1. It contains a $\mathrm{CF}_{4}$ gas radiator, providing PID from approximately 15 to $\geq 100 \mathrm{GeV} / \mathrm{c}$ for particles within the reduced polar angle acceptance of $\pm 120 \mathrm{mrad}$ (horizontal) and $\pm 100 \mathrm{mrad}$ (vertical). Two schematics and a photograph of the RICH 2 detector are shown in figure 6.4. It is aligned vertically, with its front face positioned at $9500 \mathrm{~mm}$ from the interaction point and with a depth of $2332 \mathrm{~mm}$. The overall design had to comply with the following constraints:

- the supporting structures and the photon detectors need to be placed outside the acceptance of the spectrometer and the HPDs are located left and right of the beamline where the iron

\footnotetext{
${ }^{5}$ HERAEUS Suprasil 2B.

${ }^{6}$ Vector Fields plc, Oxford, UK.

${ }^{7}$ ARMCO - Stabilized iron; $\mathrm{C} \leq 0.01 \%, \mathrm{~S}=0.01 \%, \mathrm{Mn} \leq 0.06 \%$, Si: traces, $\mathrm{P}=0.01 \%$.
} 


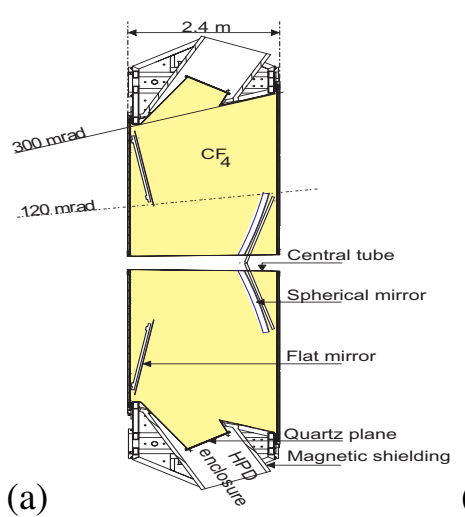

(b)

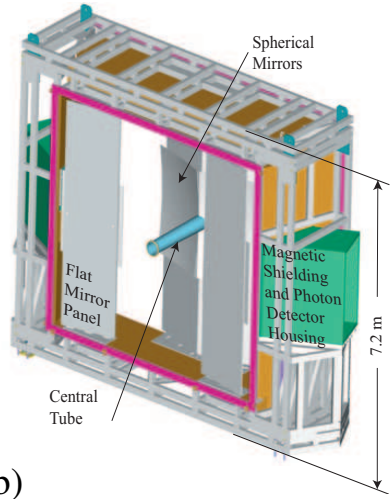

$\downarrow(\mathrm{c})$

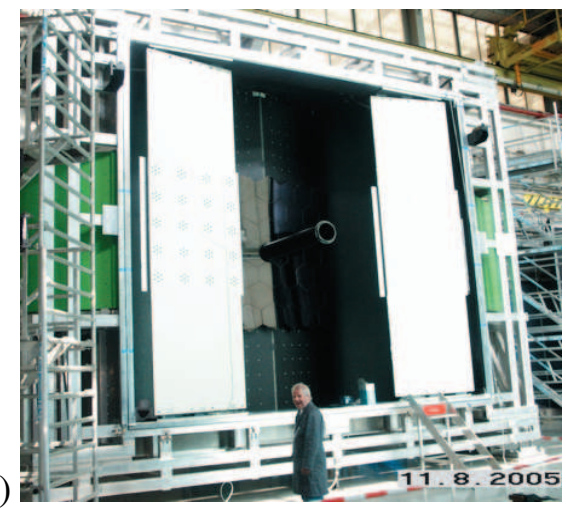

Figure 6.4: (a) Top view schematic of the RICH 2 detector. (b) A schematic layout of the RICH 2 detector. (c) A photograph of RICH 2 with the entrance window removed.

shielding is accommodated. To shorten the overall length of the detector, the reflected images from tilted spherical mirrors are reflected by flat secondary mirrors onto the detector planes. The requirement that the photon detectors are situated outside the full LHCb acceptance defines the lateral dimensions of the detector. The total radiation length of RICH 2, including the gas radiator, is about $0.15 \mathrm{X}_{0}$.

- the lower angular acceptance of the RICH 2 detector, $15 \mathrm{mrad}$, is limited by the necessary clearance of $45 \mathrm{~mm}$ around the beampipe. This distance is required to accommodate the heating jacket and thermal insulation which is required for the bakeout of the vacuum chamber (chapter 3). To gain mechanical stability of RICH 2 and minimize the material in the acceptance of the spectrometer, the detector does not split in two halves along the $x=0$ plane.

- as for RICH 1, the HPDs are located in large iron boxes in order to be shielded from the fringe field of the LHCb dipole.

\section{Optical system}

The final adjustment of the optical layout of RICH 2 has been performed with the aid of simulation, in a similar way to that described in section 6.1.1. This involves defining the position and radius of curvature of the two spherical mirror planes, the position of the two flat mirror planes, and the position of the two photon detector planes. The smearing of the reconstructed Cherenkov angle distribution provides a measure of the quality of the focusing. The RMS of the emission-point error should be small compared to the other contributions to the Cherenkov angle resolution such as the pixelization of the photon detectors and the chromatic dispersion of the radiator. The latter effect is the limiting factor for the resolution in RICH 2 , and corresponds to an uncertainty of $0.42 \mathrm{mrad}$ on the Cherenkov angle per photon [91]. The optical elements of RICH 2 must therefore be set such that the emission-point error is small compared to this value.

The parameters resulting from the optimization procedure have been adopted for the engineering design of RICH 2. The spherical mirrors have radius of curvature $8600 \mathrm{~mm}$ with centres of 

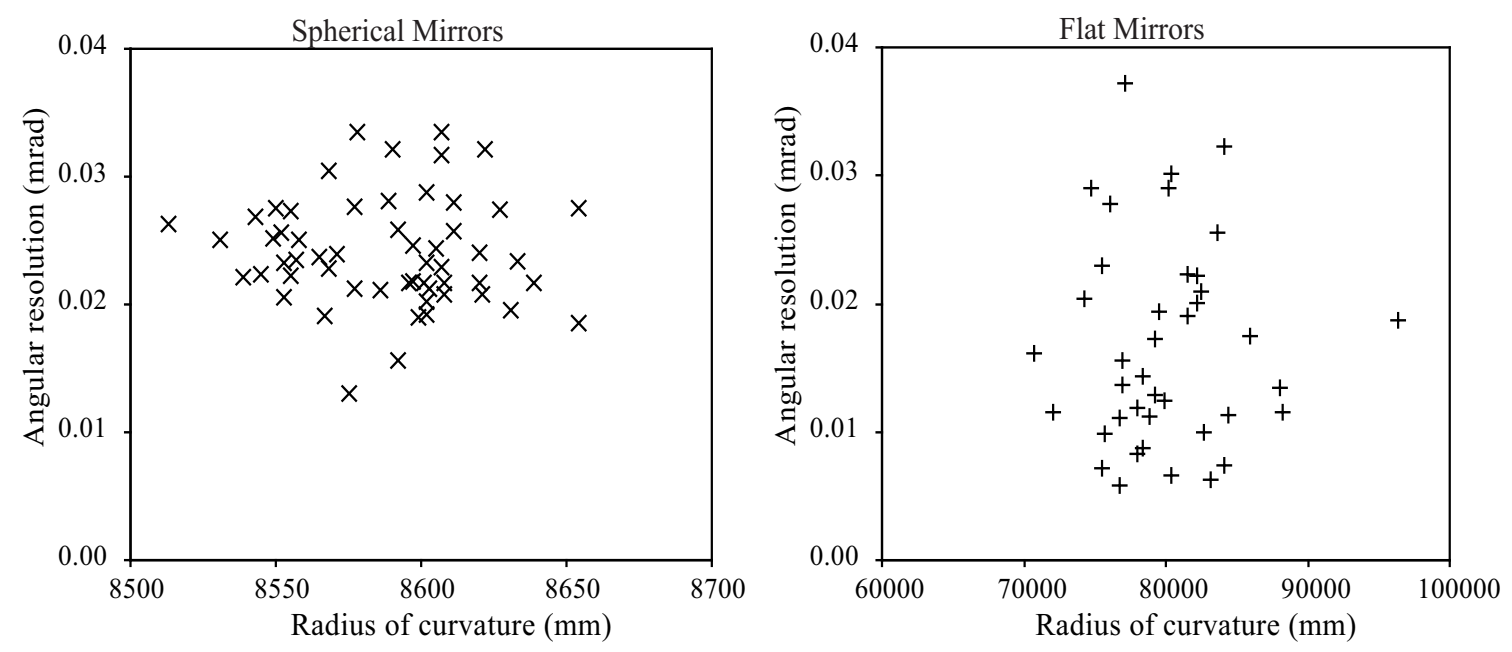

Figure 6.5: Angular precision as a function of radius of curvature for the spherical and the flat mirrors in RICH 2. The angular precision of a mirror is defined as the RMS angular deviation of the normal to the mirror surface at the given point from the radius of curvature $\mathrm{R}$, and is related to $D_{0}$ by the expression $D_{0} / 8 R$, under the assumption that the light distribution of the spot is gaussian.

curvature at $x, y, z= \pm 3270,0,3291 \mathrm{~mm}$ which defines the mirror tilt. The flat mirrors are tilted at an angle $0.185 \mathrm{rad}$ with respect to the $x$-axis, with vertical edges lying closest to the beam line located at $x, z= \pm 1234,9880 \mathrm{~mm}$. The two HPD planes are centered at $x, y, z= \pm 3892,0,10761 \mathrm{~mm}$ and tilted at an angle $1.065 \mathrm{rad}$ with respect to the $x$-axis. They are each covered with 9 rows of 16 HPDs, hexagonally close packed, with centres separated by $89.5 \mathrm{~mm}$. This results in two detector planes of $710 \mathrm{~mm} \times 1477 \mathrm{~mm}$ each.

\section{Spherical and flat mirrors}

There are two spherical mirror surfaces and two planes of flat mirrors, assembled either side of the beamline. The mirror substrates are made from $6 \mathrm{~mm}$ thick Simax glass; the development of these mirrors is described elsewhere [93-96]. The spherical mirrors are composed of hexagonal mirror elements with a circumscribed diameter of $510 \mathrm{~mm}$, and there are 26 mirrors (or half-mirrors) in each plane. The flat mirror surfaces are formed from 20 rectangular mirror segments in each plane, each $410 \times 380 \mathrm{~mm}^{2}$ in area. The greatest challenge for the manufacturers was the stability of the thin flat mirror substrates, leading to highly astigmatic or edge deformations. We have therefore chosen to use as flat mirrors, substrates with a finite but large radius of curvature, of about $80 \mathrm{~m}$. The measured properties of the mirrors are shown in figure 6.5. The impact on the resolution is discussed in section 6.1.8.

\section{Mirror support and alignment}

The mirror supports are the crucial elements that will allow the construction of a near perfect reflective surface from the individual mirror segments; the initial alignments of the mirrors must be better than $1 \mathrm{mrad}$ to have a negligible effect on the Cherenkov ring reconstruction [89]. Each 
mirror substrate is connected to a three-point adjustment system via polycarbonate flanges and rods [92]. The adjustment mechanism is attached to large aluminium sandwich panels which are fastened to the top and the bottom of the superstructure. These panels are made from two $1 \mathrm{~mm}$ thick aluminium skins separated by $28 \mathrm{~mm}$ aluminium honeycomb, ${ }^{8}$ corresponding to $\sim 4 \% \mathrm{X}_{0}$. This choice of material is again a trade-off between a long-term stability requirement, reduction of the radiation length and fluorocarbon compatibility. The mirror-support system has been tested in the laboratory for more than one year and, after an initial relaxation period, it is stable to within $100 \mu \mathrm{rad}[94,96]$. The fluctuations are mainly governed by temperature variations.

The mirrors have been installed and aligned in a three-step process [97]. First, all of the spherical mirror segments were aligned to within $50 \mu \mathrm{rad}$ of their common focal point. Then a few flat mirrors were aligned together with the coupled spherical mirrors. The final step was to align the rest of the flat mirrors with respect to these. The total error on the alignment is of the order of $100 \mu \mathrm{rad}$. Even though the fully equipped RICH 2 detector was transported by road from the laboratory to the experimental area, no change of the alignment larger than $300 \mu \mathrm{rad}$ of any mirror has been observed.

\section{RICH 2 structure}

The total weight of the RICH 2 detector is about 30 tons, a large fraction of which, $\sim 12$ tons, is the overall magnetic shielding structure. The superstructure provides the mechanically stable environment for the optical system, the overall magnetic shielding containing the photon detectors, and a lightweight configuration for the radiator gas enclosure. It is a rectangular box-shaped structure made from welded aluminium alloy rectangular hollow box sections. The deflection of the top of the structure is measured to be $<100 \mu \mathrm{m}$ under the influence of the magnetic load. The RICH 2 detector is placed and surveyed into position as one unit on the beam line and, after this, the beampipe is installed.

\section{Gas enclosure}

The total volume of the gas enclosure is about $95 \mathrm{~m}^{3}$, defined by the superstructure, the entrance and the exit windows. The entrance window, constituting a radiation length of $1.0 \% \mathrm{X}_{0}$, is a low mass composite panel made from two $1 \mathrm{~mm}$ thick carbon fibre reinforced epoxy skins, separated by $28 \mathrm{~mm}$ thick polymethacrylimide (PMI) foam. The exit window, constituting a radiation length of $2.5 \% \mathrm{X}_{0}$, is similarly made from two $1 \mathrm{~mm}$ thick aluminium skins separated by $30 \mathrm{~mm}$ PMI foam. The choice of core thicknesses and skin materials is a compromise between radiation length and deflection due to hydrostatic pressure exerted by the Cherenkov gas. The latter will be controlled to within ${ }_{-100}^{+200} \mathrm{~Pa}$ at the top of the detector. The two windows are clamped and sealed onto the superstructure and are connected to each other by a castellated central tube running coaxial to the vacuum chamber; the tube is made from $2 \mathrm{~mm}$ thick carbon fibre epoxy composite. The diameter of the tube at the entrance window is $284.5 \mathrm{~mm}$ and $350.5 \mathrm{~mm}$ at the exit.

The two planes of photon detectors are separated from the Cherenkov gas by quartz windows on each side. Each window is made from three quartz panes glued together. Each quartz pane

\footnotetext{
${ }^{8}$ Euro-Composites S.A, Zone Industrielle - B.P. 24, 6401 Echternach.
} 
is $740 \mathrm{~mm}$ by $1480 \mathrm{~mm}$ and $5 \mathrm{~mm}$ thick, with the light opening being $720 \mathrm{~mm}$ by $1460 \mathrm{~mm}$. The quartz plates have the same antireflective coating as for RICH 1 (section 6.1.1).

\section{Magnetic shielding structure}

The RICH 2 detector is positioned almost halfway between the 1600 ton iron yoke of the dipole and the massive ferromagnetic structure of the hadron calorimeter, shown in figure 2.1. The maximum stray field in the region where the photon detectors are located is more than $15 \mathrm{mT}$ and is rapidly varying in all directions [98]. The magnetic shield boxes, which are bolted onto both sides of the RICH 2 structure, must provide a mechanically stable and light tight environment for the photon detectors and attenuate the magnetic stray field by a factor of $\geq 15$. The shielding, shown in figure 6.4, is a trapezoidal structure made from $60 \mathrm{~mm}$ thick ARMCO iron plates. The residual magnetic field measured at the position of the photon detector plane is between 0.2 and $0.6 \mathrm{mT}$, to be compared to the simulated value of $\leq 0.4 \mathrm{mT}$.

\subsubsection{Radiators}

Aerogel is the ideal radiator to cover the very difficult range of refractive indices between gas and liquid. Silica aerogel is a colloidal form of quartz, solid but with an extremely low density. Its refractive index is tuneable in the range 1.01-1.10, and is ideal for the identification of particles with momentum of a few $\mathrm{GeV} / \mathrm{c}$. Aerogel has a long-established use in threshold Cherenkov counters. The development of high quality very clear samples [99] has allowed its use in RICH detectors. The dominant cause of photon loss within aerogel is Rayleigh scattering; this leads to the transmission of light with wavelength $\lambda$ through a block of thickness $L$ being proportional to $e^{-C L / \lambda^{4}}$, where the clarity coefficient, $C$, characterizes the transparency of the sample. Large hygroscopic silica aerogel [99] tiles of dimension $20.0 \times 20.0 \times 5.1 \mathrm{~cm}^{3}$ have been produced and tested for the LHCb experiment [100]. The refractive index is 1.030 at $\lambda=400 \mathrm{~nm}$ and the clarity is below $C=0.0054 \mu \mathrm{m}^{4} / \mathrm{cm}$. The effect of scattering in the aerogel dominates at high energy, so a thin $(0.30 \mathrm{~mm})$ window of glass is placed after the aerogel to absorb the photons with $E_{\gamma}>3.5 \mathrm{eV}$. This serves to reduce the chromatic aberration.

For a track passing through $5 \mathrm{~cm}$ of aerogel with $n=1.03$, the resulting number of detected photoelectrons in a saturated ring $(\beta=1)$ is expected to be $\sim 6.5$, calculated by MonteCarlo considering the detailed geometrical setup of the optics of RICH 1 and the wavelength response of the photon detectors. The MonteCarlo was found to describe reasonably well the number of photoelectrons measured in a test beam [100]. Tests have shown [101] that the optical properties will not degrade significantly over the timescale of the LHCb experiment. The aerogel is stable against intense irradiation and shows no significant change in transparency once tested after an accumulated fluence of up to $5.5 \times 10^{13} / \mathrm{cm}^{2}$ of neutrons or protons, nor after a $\gamma$ dose of $2.5 \times 10^{5} \mathrm{~Gy}$. It is sensitive to water vapour absorption, but its transparency can be restored after a bakeout of the tiles. The volume of aerogel required is modest, $\sim 30 \ell$, so its replacement, if required, would be relatively straightforward. The refractive index is fairly uniform across a tile, despite the large transverse dimension and thickness. The spread has been measured [102] to be $\sigma_{n-1} /(n-1) \sim 0.76 \%$. In RICH 1 the aerogel sits in the $\mathrm{C}_{4} \mathrm{~F}_{10}$ gas radiator. Tests have shown that $\mathrm{C}_{4} \mathrm{~F}_{10}$ does not significantly degrade the aerogel performance [103]. 
The fluorocarbon gases $\mathrm{C}_{4} \mathrm{~F}_{10}(\mathrm{RICH} 1)$ and $\mathrm{CF}_{4}(\mathrm{RICH} 2)$ were chosen because their refractive indices are well matched to the momentum spectrum of particles from $B$ decays at $\mathrm{LHCb}$ and because they have a low chromatic dispersion. The refractive indices at $0^{\circ} \mathrm{C}$ and $101325 \mathrm{~Pa}$ are parameterized by:

$$
C_{4} F_{10}:(n-1) \times 10^{6}=0.25324 /\left(73.7^{-2}-\lambda^{-2}\right)
$$

and

$$
C F_{4}:(n-1) \times 10^{6}=0.12489 /\left(61.8^{-2}-\lambda^{-2}\right)
$$

where the photon wavelength $\lambda$ is in $n$ [104]. For $\mathrm{C}_{4} \mathrm{~F}_{10}, \mathrm{n}=1.0014$ and for $\mathrm{CF}_{4}, \mathrm{n}=1.0005$ at $\lambda=400 \mathrm{~nm}$. The effective radiator lengths are about $95 \mathrm{~cm}$ in $\mathrm{C}_{4} \mathrm{~F}_{10}$ and $180 \mathrm{~cm}$ in $\mathrm{CF}_{4}$. The estimated photoelectron yield is $\sim 30$ and $\sim 22$ respectively for charged $\beta \approx 1$ particles.

The gas radiators are operated slightly above atmospheric pressure $\lesssim 50 \mathrm{~Pa}$ (measured at the top of the gas vessel) and at ambient temperature. Pressure and temperature are recorded in order to compensate variations in the refractive index (section 6.1.7). These fluorocarbons are transparent well below $150 \mathrm{~nm} ; \mathrm{CO}_{2}$ is used as a pressure balancing gas which itself is transparent down to $180 \mathrm{~nm}$ [105]. Since the quantum efficiency of the HPDs is zero below $190 \mathrm{~nm}$ (section 6.1.5), air contamination does not significantly affect the photon yield. $\mathrm{O}_{2}$ and $\mathrm{H}_{2} \mathrm{O}$ contamination are however kept low at about $100 \mathrm{ppm}$, mainly due to possible radiation-induced formation of HF. The $\mathrm{CO}_{2}$ fraction is kept constant at $\sim 1 \%$.

\subsubsection{Mirror reflectivity studies}

Several reflectivity coatings are available for RICH mirrors; the choice depends on the Cherenkov photon spectrum, the mean angle of incidence, the long term stability and compatibility to fluorocarbons. Seven different coatings have been tested [93, 106] and the reflectivity measured with a spectrophotometer at an incidence angle of $30^{\circ}$, close to the average angle with which the photons will impinge on the RICH 2 mirrors. The results are shown in figure 6.6. The reflectivity coatings for the RICH 1 mirrors have been optimized for the the different mean angles of incidence, i.e. $25^{\circ}$ for the spherical mirrors and $45^{\circ}$ for the flat mirrors.

Simulation studies have been performed by convoluting the reflectivity data with the quantum efficiency of the photon detectors, the Cherenkov photon energy spectra and the transmittance of the $\mathrm{CF}_{4}$ radiator in $\mathrm{RICH} 2$. The results of the simulation, in terms of the relative number of detected photons, are summarised in table 6.1.

The reflectivity of the two coatings with a layer of hafnium oxide $\left(\mathrm{HfO}_{2}\right)$ is very high in the near UV (curve I and II in figure 6.6), where the quantum efficiency of the photon detector is peaked. The simulation shows that good matching, taking into account the two reflections on spherical and flat mirrors, leads to a detected photon yield 5\% higher for this coating compared to other UV extended coatings with magnesium fluoride $\left(\mathrm{MgF}_{2}\right)$ as the surface layer. Magnesium fluoride coatings have been successfully used in RICH detectors with $\mathrm{C}_{4} \mathrm{~F}_{10}$ and $\mathrm{C}_{5} \mathrm{~F}_{12}$ gas radiators in the DELPHI [107] and COMPASS [108] experiments, and hafnium oxide coatings have been successfully tested in $\mathrm{C}_{6} \mathrm{~F}_{14}$ vapour. Hafnium oxide also provides a very hard and chemically inert protective layer for the mirrors. For these reasons the $\mathrm{Al}+\mathrm{SiO}_{2}+\mathrm{HfO}_{2}$ coating was chosen for all RICH 1 and RICH 2 glass mirrors. $\mathrm{SiO}_{2}$ is used for the middle layer of the multi-layer coating, and not $\mathrm{MgF}_{2}$, for technical reasons. 


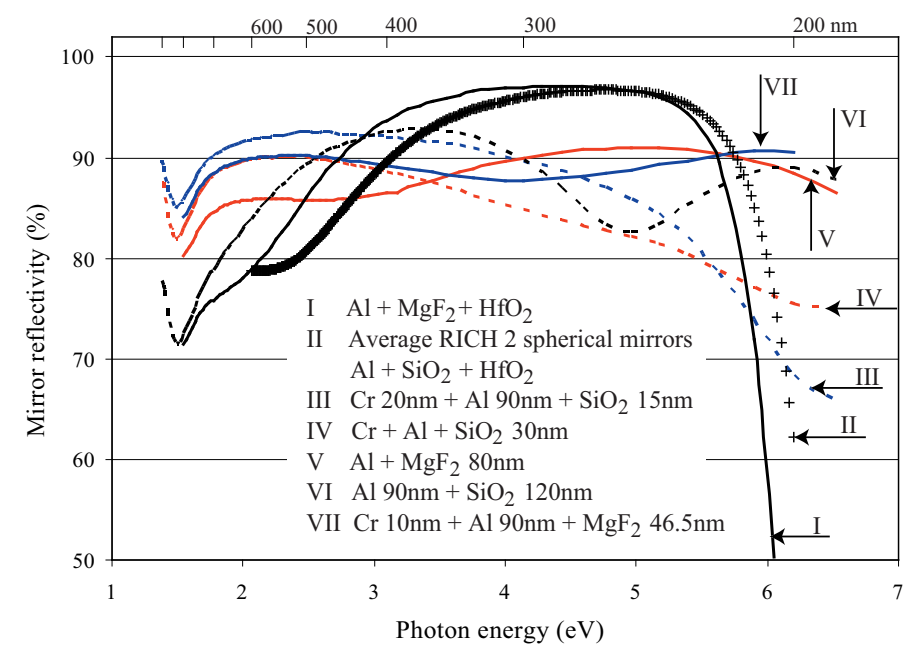

Figure 6.6: Reflectivity of several mirror coatings on glass as a function of the photon energy. The angle of incidence is $30^{\circ}$.

Table 6.1: Relative number of detected photons simulated in RICH 2 for different mirror coatings. The values are normalised to the highest photoelectron yield, set to 1 .

\begin{tabular}{|lc|}
\hline Coating & Photoelectron yield \\
\hline $\mathrm{Cr}+\mathrm{Al}+\mathrm{SiO}_{2}(30 \mathrm{~nm})$ & 0.865 \\
$\mathrm{Al}+\mathrm{SiO}_{2}$ & 0.945 \\
$\mathrm{Cr}+\mathrm{Al}+\mathrm{MgF}_{2}$ & 0.947 \\
$\mathrm{Al}+\mathrm{MgF}_{2}$ & 0.960 \\
$\mathrm{Cr}+\mathrm{Al}+\mathrm{SiO}_{2}(15 \mathrm{~nm})$ & 0.960 \\
$\mathrm{Al}+\mathrm{SiO}_{2}+\mathrm{HfO}_{2}$ & 1 \\
$\mathrm{Al}+\mathrm{MgF}_{2}+\mathrm{HfO}_{2}$ & 1.00 \\
\hline
\end{tabular}

\subsubsection{Photon Detectors}

\section{Pixel Hybrid Photon Detector}

The RICH detectors utilize Hybrid Photon Detectors (HPDs) to measure the spatial positions of emitted Cherenkov photons. The HPD is a vacuum photon detector in which a photoelectron, released from the conversion in a photocathode of an incident photon, is accelerated by an applied high voltage of typically 10 to $20 \mathrm{kV}$ onto a reverse-biased silicon detector. During the photoelectron energy dissipation process in silicon, electron-hole pairs are created at an average yield of one for every $3.6 \mathrm{eV}$ of deposited energy. Carefully-designed readout electronics and interconnects to the silicon detector result in very high efficiency at detecting single photoelectrons.

A dedicated pixel-HPD has been developed by LHCb, in close collaboration with industry [109]. The specific RICH requirements are a large area coverage $\left(\sim 3.5 \mathrm{~m}^{2}\right)$ with high active-to-total area ratio after close-packing (64\%), high granularity $\left(2.5 \times 2.5 \mathrm{~mm}^{2}\right.$ at the photocathode) and high speed ( $25 \mathrm{~ns}$ timing resolution). The pixel-HPD is shown in figure 6.7. It is 

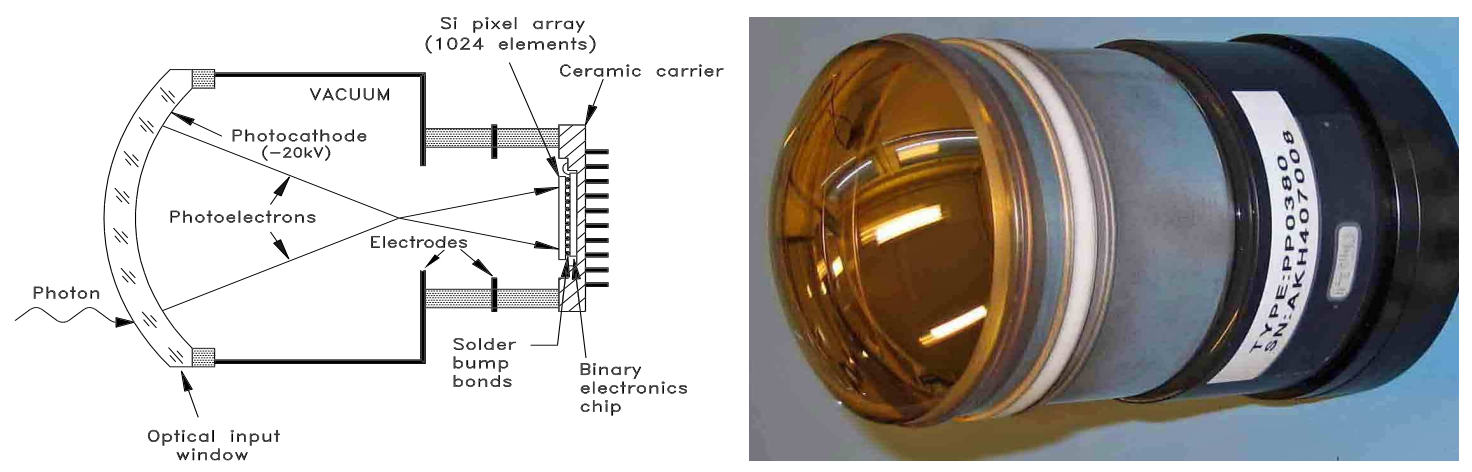

Figure 6.7: Left: a schematic and right: a photograph of the pixel-HPD.

based on an electrostatically focussed tube design with a tetrode structure, de-magnifying by a factor of $\sim 5$ the photocathode image onto a small silicon detector array. The silicon detector is segmented into 1024 pixels, each $500 \mu \mathrm{m} \times 500 \mu \mathrm{m}$ in size and arranged as a matrix of 32 rows and 32 columns. This leads to the required pixel size at the HPD entrance window of $2.5 \times 2.5 \mathrm{~mm}^{2}$. The nominal operating voltage of the HPD is $-20 \mathrm{kV}$, corresponding to $\sim 5000$ electron-hole pairs released in the silicon.

The silicon pixel detector array is bump-bonded to a binary readout chip (section 6.1.6). This flip-chip assembly is mounted and wire-bonded onto a Pin Grid Array (PGA) ceramic carrier, forming the HPD anode. Since all anode parts are encapsulated in vacuum, they must be compatible with the vacuum tube technologies, and must stand high $\left(300^{\circ} \mathrm{C}\right)$ bakeout temperatures. In particular, a specific fine-pitch bump-bonding process has been developed for this application. ${ }^{9}$ The HPD entrance window is fabricated from quartz and forms a spherical surface, with $7 \mathrm{~mm}$ thickness and $55 \mathrm{~mm}$ inner radius of curvature. The photocathode is of the thin-S20 multi-alkali type, deposited on the quartz inner surface. Normally-incident Cherenkov photons to the HPD plane can be detected over an active diameter of $75 \mathrm{~mm}$ and, since the overall tube diameter is $83 \mathrm{~mm}$, the intrinsic tube active area fraction is $(75 / 83)^{2}=0.817$. A total of 484 tubes (196 for RICH1 and 288 for RICH2) are required to cover the four RICH photon detection surfaces.

The demagnification by 5 of the photoelectron image is achieved by biasing the photocathode at $-20 \mathrm{kV}$, the first electrode at $-19.7 \mathrm{kV}$ and the second electrode at $-16.4 \mathrm{kV}$. The RMS values for the point spread function (PSF) at the pixel array are constant over the tube radius and equal to $80 \mu \mathrm{m}$ for red light and $180 \mu \mathrm{m}$ for blue-near UV light, in the absence of magnetic field.

\section{HPD test results}

The HPDs have been fabricated in industry ${ }^{10}$ and were then qualified at two test facilities to determine their efficiency and optimum working parameters, before installation at CERN. Each HPD in turn was placed in a light-tight box, illuminated with an LED of wavelength $470 \mathrm{~nm}$, and read out by custom electronics. A selection of test results is presented below. Measurements of the quantum efficiencies (QEs) were carried out after manufacture at Photonis-DEP. Measurements were then

\footnotetext{
${ }^{9}$ VTT, Finland.

${ }^{10}$ Photonis-DEP, Roden, Netherlands.
} 


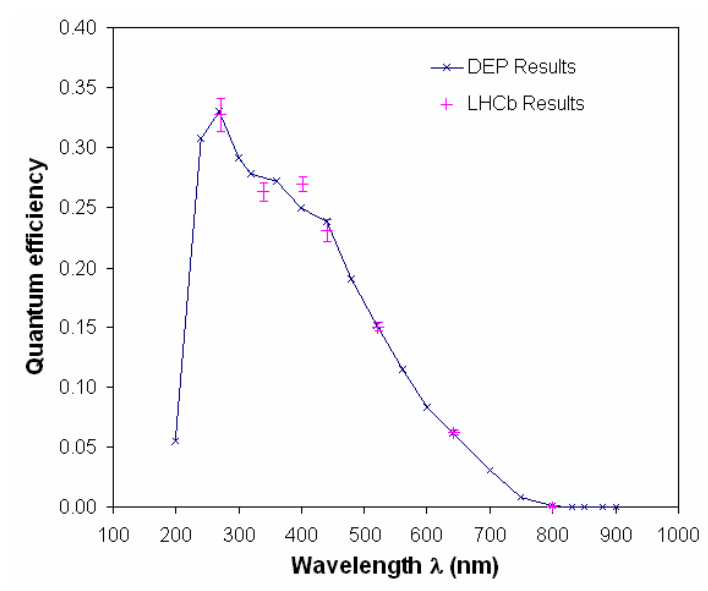

Figure 6.8: $\mathrm{QE}$ measurement for one of the best HPD.

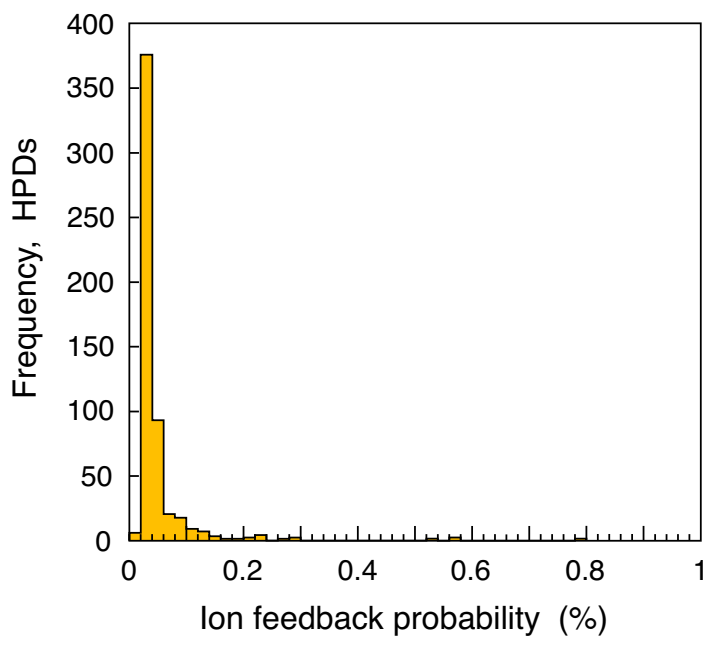

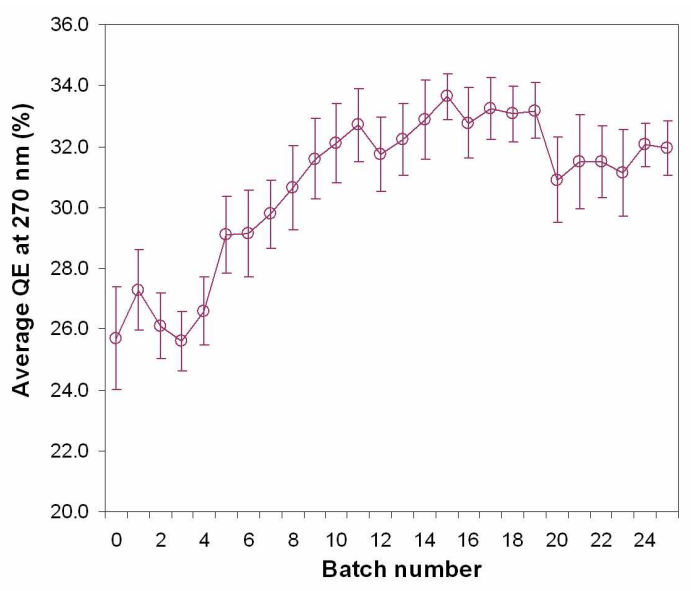

Figure 6.9: The average $\mathrm{QE}(\%)$ at $270 \mathrm{~nm}$ versus the HPD batch number.

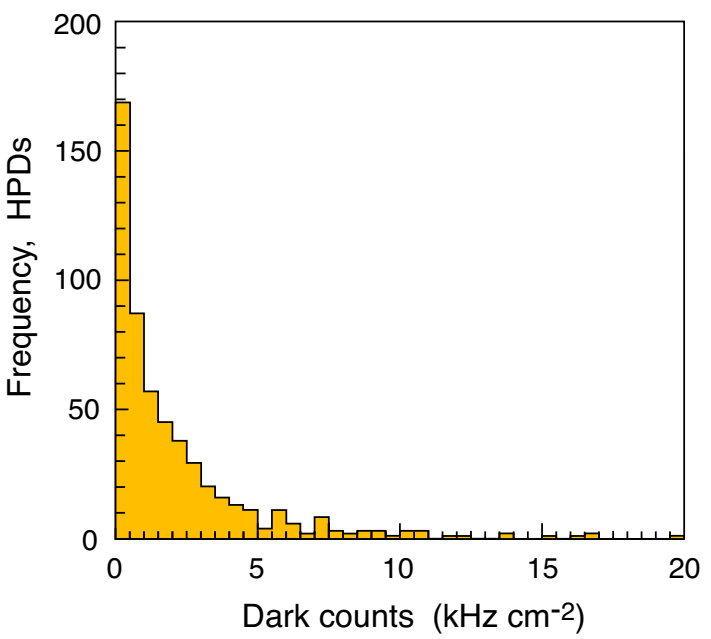

Figure 6.10: Distributions of the ion-feedback rate (left) and of the dark-count rates (right).

repeated at the test facilities for a subsample of $10 \%$ of the HPDs, using a calibrated photodiode and a quartz-tungsten halogen lamp. The QE curve for one of the best tubes is shown in figure 6.8. The average QE value per HPD batch number and the running average QE value are shown in figure 6.9 for all HPDs (484 tubes plus 66 spares). The QE curves show an average maximum of $31 \%$ at $270 \mathrm{~nm}$, considerably above the specification minimum of $20 \%$.

The vacuum quality in the HPD tube is determined by measuring ion feedback. During the acceleration process, photoelectrons may hit residual gas atoms, producing ions. These drift to the photocathode, releasing 10-40 electrons which are detected 200-300 ns after the primary photon signal. Figure 6.10 shows that the ion feedback rate is well below the specification maximum of $1 \%$, indicating that the vacuum quality is excellent.

An important quality factor of an HPD is low dark-count rate, the main sources being thermionic electron emission at the photocathode, electrostatic field emission and any resulting 


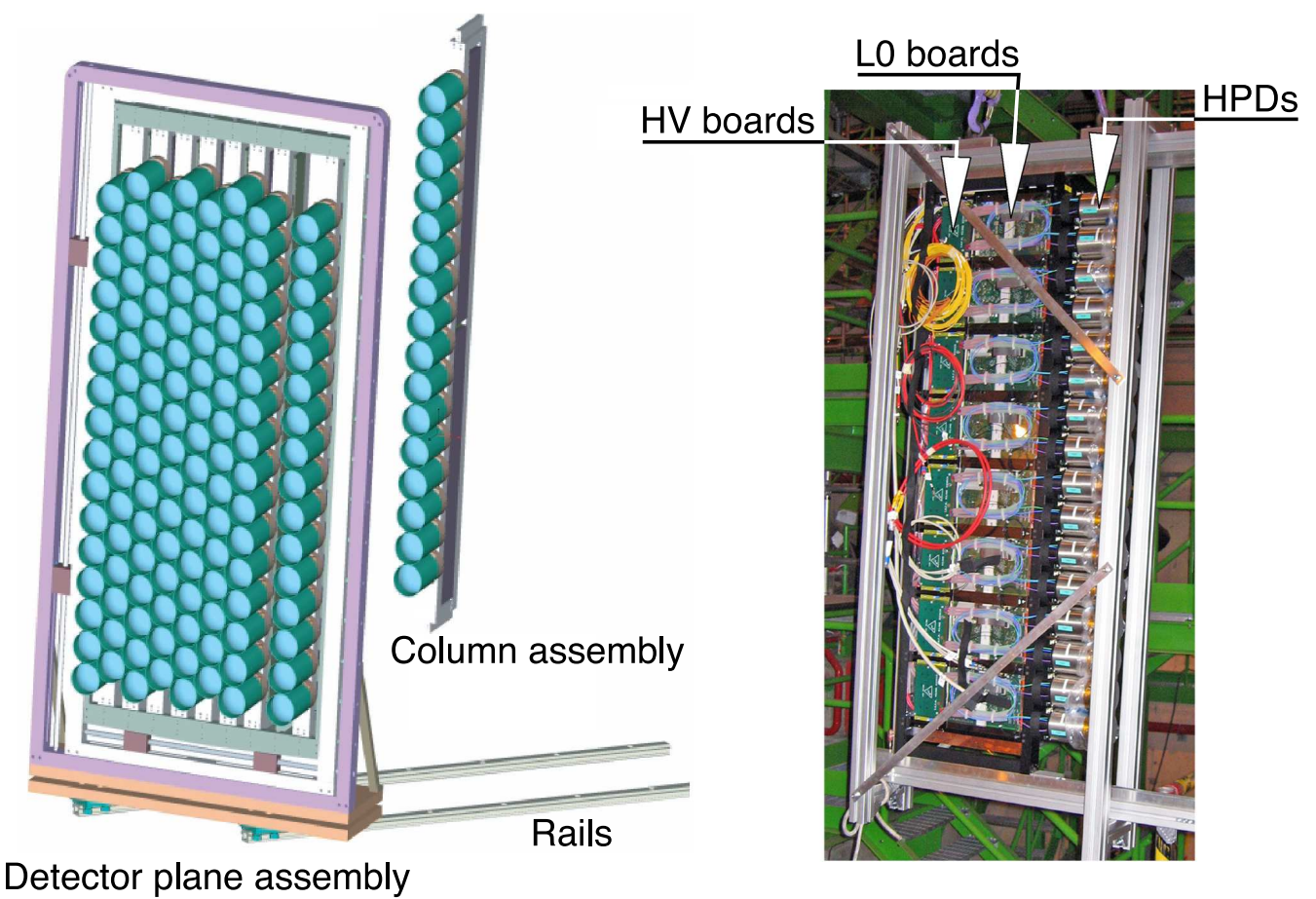

Figure 6.11: Left: the RICH 2 column mounting scheme and right: a photograph of a column.

ion feedback. Figure 6.10 shows the measured dark-count rates in the sample of HPDs in a run with the LEDs off. Dark-count rates are typically below the specification value, $5 \mathrm{kHz} / \mathrm{cm}^{2}$ with respect to the photocathode area, which in turn is $\sim 10^{3}$ less than the maximum occupancy expected in the RICH detectors.

HPD testing was carried out at a rate of 30-40 HPDs per month. A total of $97 \%$ have been found to meet or exceed the design criteria in key areas.

\section{Photon Detector integration}

The HPDs are grouped in four detection planes (two for RICH1 and two for RICH2) and positioned on a hexagonal lattice. The hexagonal close-packing factor is 0.907 . Each tube is completely surrounded by a $1 \mathrm{~mm}$ thick cylindrical magnetic shield which protects against stray external B-fields up to $5 \mathrm{mT}$ (the maximum field value within the RICH 1 magnetic shielding has been measured to be $2.4 \mathrm{mT}$, see section 6.1.1). A tube-to-tube pitch of $89.5 \mathrm{~mm}$ in both RICH detectors has been chosen, resulting in a packing factor of $(75 / 89.5)^{2} \times 0.907=0.64$. The HPDs are mounted on columns which are installed in the magnetic shielding boxes of the two RICH detectors. There are $2 \times 7$ columns of HPDs in RICH 1 with 14 HPDs per column and $2 \times 9$ columns in RICH 2 with 16 HPDs per column. Figure 6.11 illustrates the column mounting scheme for RICH 2. The column also contains front-end electronics boards (one per pair of HPDs), power supply distribution, cabling and active cooling, all within one mechanical module. The electronics boards and power distribution are described in section 6.1.6. 


\subsubsection{Electronics}

The RICH electronics system reads out data from the HPDs (about $5 \times 10^{5}$ channels) and conforms to the general electronics architecture of $\mathrm{LHCb}$ (see section 2.2). The electronics system is divided into so-called Level-0 (L0) and Level-1 (L1) regions. The L0 electronics are all located on-detector where they will be exposed to radiation and must therefore contain only radiation-qualified components. The $\mathrm{L} 1$ electronics modules are housed in the counting house behind the radiation-shield wall and hence are not required to be radiation tolerant.

\section{Level-0 electronics}

The L0 electronics comprises the pixel chip, ZIFs/kaptons, L0 board and LV/HV distribution, and is described below.

Pixel Readout Chip: At the beginning of the electronics readout chain is the LHCBPIX1 pixel chip [110] that forms part of the anode assembly of the HPD (section 6.1.5). The chip has been designed in a commercial $0.25 \mu \mathrm{m}$ CMOS process using special layout techniques to enhance its resistance to radiation. The chip is connected to a silicon pixel sensor by an array of solder bumpbonds, one per channel. Each pixel measures $62.5 \mu \mathrm{m}$ by $500 \mu \mathrm{m}$ and 8192 pixels are arranged as a matrix of 32 columns by 256 rows. Circuitry on the chip logically ORes eight adjacent pixels, thus transforming the matrix into 1024 channels of 32 by 32 , each of size $500 \mu \mathrm{m}$ by $500 \mu \mathrm{m}$. Signals from the silicon sensor are amplified, shaped and then compared with a global threshold. Hits are buffered for the duration of the Level-0 trigger latency and triggered events are then read out by way of a 16-deep multi-event FIFO buffer. The 32 columns are read out in parallel. The analog behaviour of the chip is crucial for a good photodetection efficiency, in particular low noise and uniform threshold. Measured values are well within the HPD specifications, with typically 160 electrons noise, compared to a signal of 5000 electrons, and a mean threshold of 1200 electrons with RMS spread of 100 electrons.

ZIFs and Kaptons: Signals to and from the HPD pass through the pins of the ceramic pin-gridarray carrier. This is plugged into a 321-pin Zero-Insertion-Force (ZIF) connector mounted on a small circuit board. The board also contains passive components for filtering and line-termination. Signals are then transmitted on two Kapton cables which also carry the low-voltage power to the HPD. These Kaptons allow mechanical flexibility between the mounting of the HPDs and the other Level-0 components.

LO Board: The L0 board [111] acts as an interface between the HPDs and the ECS (Experiment Control System), TFC (Timing and Fast Control) and data transmission systems. This interfacing is implemented in the Pixel Interface (PINT). The antifuse gate array, ACTEL AX1000, was chosen for its tolerance to single event effects (single event latch-up and single event upset). Its main tasks are to receive data from two HPDs, add headers containing event information and data-integrity checks, and transfer events to the data transmission system. A total of $242 \mathrm{~L} 0$ boards are used in the RICH detectors. A photograph of a single board is shown in figure 6.12. The tolerance of the PINT to single event effects was verified by irradiation tests to simulate the expected dose in the LHCb environment. The measured rate of single event upset (SEU) was negligible, no cases of latch-up were observed and chips survived many times the expected dose of ionising radi- 


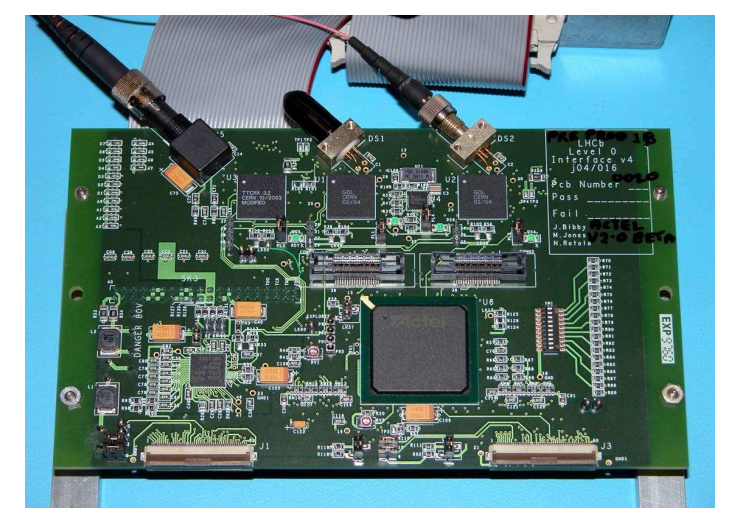

Figure 6.12: A photograph of the L0 board. The optical receiver and transmitters are visible at the top. The PINT gate array is in the centre of the board.

ation. All other components on the L0 board have been designed in radiation-hardened or tolerant technologies already qualified to levels far in excess of the RICH environment.

The optical data transmission consists of Gigabit Optical Link (GOL) chips [112] and Vertical Cavity Surface Emitting Lasers (VCSEL). The GOLs serialise the data into a $1.6 \mathrm{Gbit} / \mathrm{s}$ bit stream using $8 \mathrm{~b} / 10 \mathrm{~b}$ encoding and the VCSELs convert this into an optical signal of $850 \mathrm{~nm}$ wavelength. Two output fibres, one per HPD, transmit the data from each $\mathrm{L} 0$ board. At a $1 \mathrm{MHz}$ trigger rate, the aggregate output data rate from the RICH photon detectors is in the region of $500 \mathrm{Gbit} / \mathrm{s}$ distributed over 484 optical fibres. Also on the board is a TTCrx [113] which generates the $40 \mathrm{MHz}$ clock and trigger and calibration signals. The input to the TTCrx arrives on fibres connected to the global TFC system. Finally, a chip known as the Analog-Pilot generates reference voltages required by the HPDs and digitizes monitoring signals such as the temperature of the board as measured by PT1000 sensors.

LV/HV Distribution: The low voltages required by the HPDs and the L0 boards are provided by the LV distribution system. These voltages are generated locally using radiation-tolerant linear voltage regulators mounted on the LV boards. Each LV board can power two L0 boards independently, and four LV boards are daisy-chained together in an HPD column. The top-most contains a SPECS slave module [41] which provides the interface for the configuration of the HPDs and L0 boards in that column. The SPECS interface is also used for switching on and off the voltage regulators, thus allowing careful control of the powering of the column.

The high voltages for the HPDs are provided by the HV distribution system [114]. As shown in figure $6.13 \mathrm{~b}$, the three voltages are derived from a $20 \mathrm{kV}$ input by means of a resistive divider, one per half-column, and short circuit protection is provided by $1 \mathrm{Gohm}$ resistors in series with each HPD voltage line. Various measures have been taken to minimise the risk of discharge or breakdown. The bare PCB of the HV cards is machined to introduce cuts around critical points which are then filled with dielectric gel to improve insulation. After component mounting and cabling, the entire assembly is encapsulated in silicone rubber. A photograph of a completed board is shown in figure 6.13. A total of 242 boards are used to equip the RICH detectors.

Figure 6.14 shows a photograph of all front-end electronics components mounted on a RICH HPD column. On the left are the Kapton cables shown plugged into the L0 boards, then comes a 

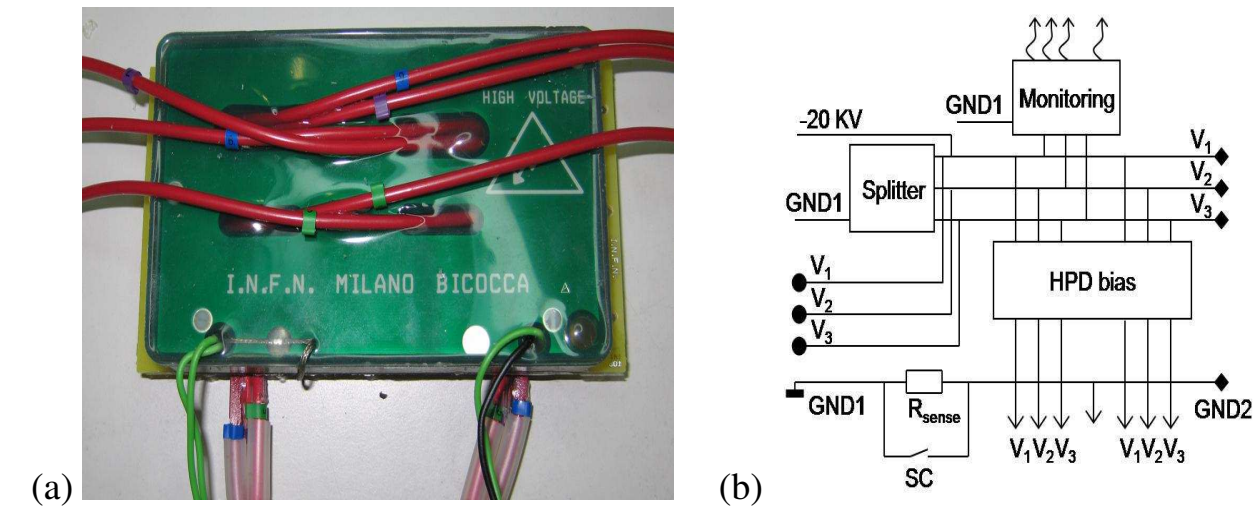

Figure 6.13: A photograph (a) and the scheme (b) of an HV board. Around the board is the silicone coating.

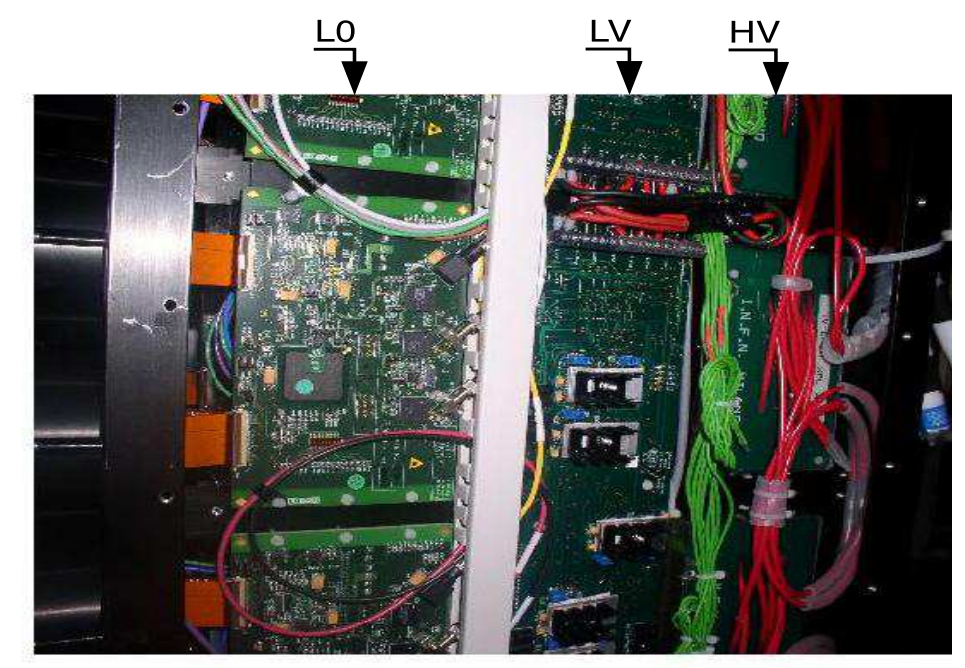

Figure 6.14: A photograph of LO, LV and HV boards mounted on a RICH HPD column.

cable tray to carry the optical fibres. Next comes the LV board which has heat sinks mounted on the surface of the voltage regulators. At the far right are the HV boards.

\section{Level-1 electronics}

The RICH L1 electronics, located off-detector in the counting room, has been designed to implement data compression and also to serve as the interface between the custom data transmission protocol of the L0 electronics and the industry-standard Gbit Ethernet protocol used by the DAQ network. The off-detector electronics also performs the important function of isolating the DAQ network from errors induced in the L0 data due to radiation induced SEU.

Each incoming serial data channel is first converted from optical to electrical using Agilent HFBR-782 optical receivers. The serial electrical data are then AC coupled to dedicated I/O pins of Virtex2Pro FPGAs, where they are deserialised using integrated Gbit transceivers that are com- 
patible with the data encoding used by the GOL serialisers in the L0 electronics. All further data processing is done in the FPGA programmable logic.

The L1 electronics modules are controlled by signals broadcast synchronously to all subdetectors by the readout supervisor. These signals are used to control the generation of the data packets sent by the L1 to the DAQ network (see section 8.3). The data content of these packets is extracted from the incoming L0 data frames. The generation of the data packets operates autonomously to the arrival of the L0 data and therefore cannot be disrupted by incoming erroneous data. In order to predict the time of arrival of the $\mathrm{L} 0$ data frames so that they can be correctly inserted into the generated data packets, the operation of the L0 electronics is emulated in the off-detector modules using the TFC broadcast signals.

Once the incoming data frames have been received, the zero-suppression algorithm (L1 module copies only the non-zero bytes into the zero-suppressed buffer) is applied in parallel to all streams. The fully pipelined algorithm operates at the $1 \mathrm{MHz}$ input event rate and therefore does not introduce dead-time. Zero-suppressed data are buffered in internal memory in the four ingress FPGAs before being multiplexed into multi-HPD event packets. These packets are then forwarded to the egress FPGA which further multiplexes the data into multi-event packets and transmits them using Gbit Ethernet protocol to the DAQ network using the LHCb quad Gbit Ethernet interface.

The L1 module is configured and monitored via an LHCb Credit Card PC (CCPC) [115] interface. The $\mathrm{L} 1$ modules are in $9 \mathrm{U}$ format and each can receive data from a maximum of 36 HPDs. The input links are distributed across three 12-channel optical receivers and four Virtex2Pro ingress FPGAs. For $1 \mathrm{MHz}$ operation, the data throughput is expected to be limited by the capacity of the four $1 \mathrm{Gbit} / \mathrm{s}$ DAQ links at higher luminosity. Static load balancing is done by physically distributing the input fibre-optic cables across the modules. The bandwidth is distributed across 21 L1 boards to avoid bottlenecks and problems associated with inhomogeneous occupancies in the different parts of the RICH detectors.

\subsubsection{Monitoring and control}

The RICH Detector Control System (DCS) [116] monitors the working conditions of both RICH 1 and $\mathrm{RICH} 2$, controls the operating conditions and ensures the integration of RICHes in the LHCb detector control system. It is composed of several parts:

- Power supply control and monitoring (low voltage, silicon detector bias and very high voltage);

- Environment monitoring (temperature, pressure, humidity);

- Gas quality monitoring (gas purity);

- Magnetic distortion monitoring (and correction);

- Laser alignment monitoring system.

\section{Power supplies}

Low voltage $(5 \mathrm{~V})$ for the front-end electronic boards is provided by commercial off-the-shelf devices, the Wiener MARATON power supply. It is radiation and magnetic field tolerant, and can 
be remotely monitored via a network using standard software interfaces provided by the manufacturer (OPC server). Other lower voltages (e.g. 3.3 V) required by the electronics components are generated, regulated and monitored on each front-end LV board. In addition, to improve reliability, temperature sensors placed near hot spots and critical points allow a quick check of the integrity of the electronics. A similar solution has been adopted for biasing the HPD internal silicon pixel detectors: a standard CAEN SY1527 mainframe power supply with plug-in modules. For the very high voltage of the HPDs $(20 \mathrm{kV})$, no satisfactory commercial solution was found so the system was designed in-house. The system is built around a commercial HV module (ISEG CPn20050424 10) controlled and monitored by a pair of DACs and ADCs connected via an I2C bus interface to a CCPC running the software to regulate the voltage and to connect to the network. The CCPC, using a bare-bones version of Linux, runs the control program which, in an endless loop, checks all voltages and currents, and via a DIM protocol [117] reports measurements to the high level control system. In the unlikely case of a loss of the network connection, the CCPC can still check the working conditions and cut power to the HPDs to avoid damage to personnel and HPDs.

\section{Environment}

Environmental monitoring (temperature, pressure, humidity of the radiator gas and HPD planes) is achieved using standard commercial sensors, namely platinum resistors for temperature, diaphragm sensors for pressure and HMX2000-HT sensors (by Hygrometrix) for humidity. All these devices are mounted in the harsh radiation environment hence they must be read by radiation tolerant electronics i.e. the Embedded Local Monitor Board (ELMB) [118].

\section{Gas quality}

The purity of the gas radiator is critical to obtain good photon transparency and for this reason a quick analysis tool to spot any contamination is employed. The stability of the gas composition is checked by periodically monitoring the speed of sound of the gas in the vessel. The speed of sound is given by $v_{s}=\sqrt{\frac{\gamma R T}{M}}$ where $\gamma=\frac{C_{p}}{C_{v}}$ is the ratio of specific heats of the gas, $\mathrm{R}$ is the universal gas constant, $\mathrm{T}$ the absolute temperature and $\mathrm{M}$ the average molecular mass. The speed is monitored by measuring the time that a sound pulse takes to propagate from an electrostatic transducer to the end of a cylindrical vessel and then, reflected by the end wall, back to the same transducer, working now as a microphonic sensor. For RICH 1 this vessel is $0.5 \mathrm{~m}$ long and the measured time interval is of the order of $10 \mathrm{~ms}$ in $\mathrm{C}_{4} \mathrm{~F}_{10}$. For RICH 2 the vessel is approximately $0.3 \mathrm{~m}$ long and the relative propagation time is of the order of $3.8 \mathrm{~ms}$ in $\mathrm{CF}_{4}$. The whole system is built around a timer/counter provided by a National Instrument acquisition board. The internal counter, running at $20 \mathrm{MHz}$, provides $50 \mathrm{~ns}$ time resolution, more than adequate to detect a $1 \% \mathrm{CO}_{2}$ contamination (which will give a $50 \mu s$ change in $\mathrm{C}_{4} \mathrm{~F}_{10}$ and $30 \mu s$ change in $\mathrm{CF}_{4}$ for the total transit time). There are two such systems for each RICH detector, one placed on the inlet pipe and the second on the outlet of the fluid system. A typical measurement is shown in figure 6.15 which shows the change in the propagation time when the percentage of $\mathrm{CO}_{2}$ changes from 0 to $5 \%$. 


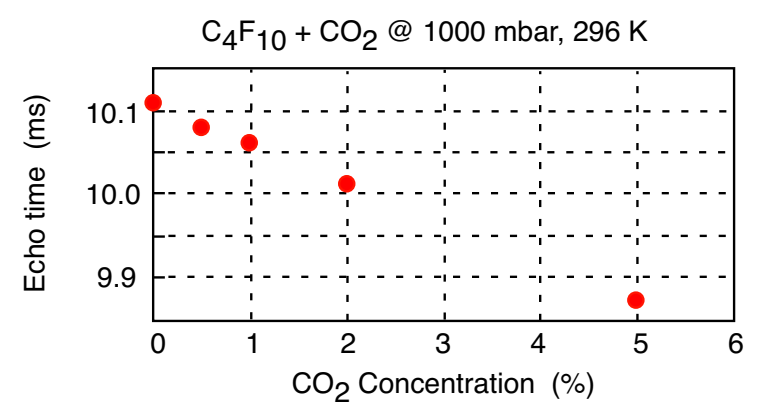

Figure 6.15: Typical sound propagation time as a function of the $\mathrm{CO}_{2}$ percentage in $\mathrm{C}_{4} \mathrm{~F}_{10}$.

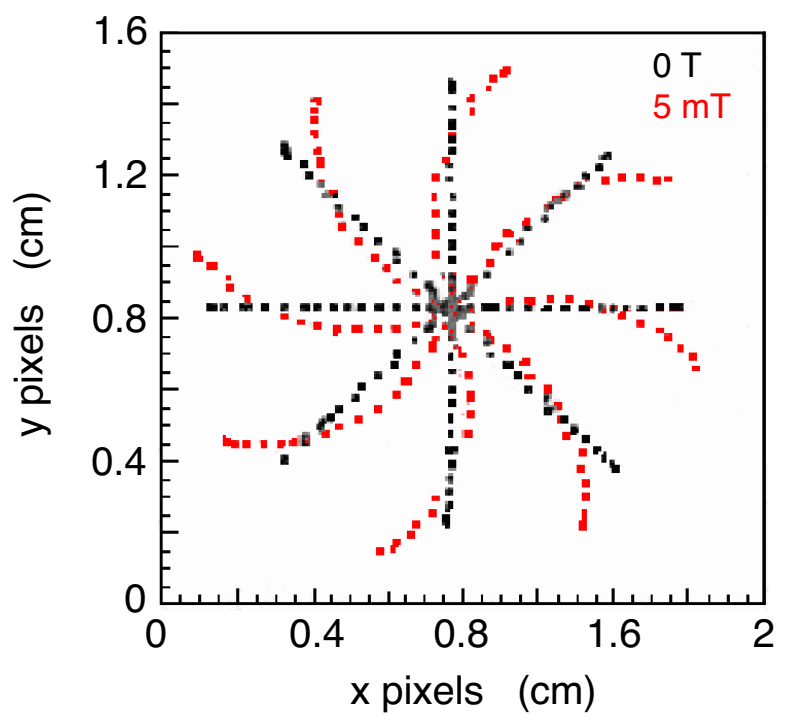

Figure 6.16: Image of a star pattern recorded on an HPD with and without a B-field of $5 \mathrm{mT}$ applied parallel to the HPD axis.

\section{Magnetic distortions}

The fringe field of the LHCb dipole magnet distorts the photoelectron trajectories within the hybrid photon detectors, hence distorting the image recorded by the pixel chip. The HPDs are individually shielded by MuMetal cylinders and the arrays are completely surrounded by iron shields, however residual stray fields (up to an expected maximum of $2.4 \mathrm{mT}$ in RICH 1) are sufficient to severely degrade the required precision. This effect can be clearly seen in figure 6.16, which shows how a star pattern recorded without B-field is badly distorted with a $5 \mathrm{mT}$ field applied parallel to the HPD axis. Hence individual correction factors are required for each HPD and the effect of the B-field must be monitored periodically. For RICH 1 an LED system mounted in the photon funnel region between the HPDs and the quartz window is used. For RICH 2 a commercial light projection system projecting a fixed pattern of dots from outside the RICH vessel will monitor the magnetic distortions. In both cases the data will be acquired during dedicated calibration runs and the magnetic corrections applied off-line. 


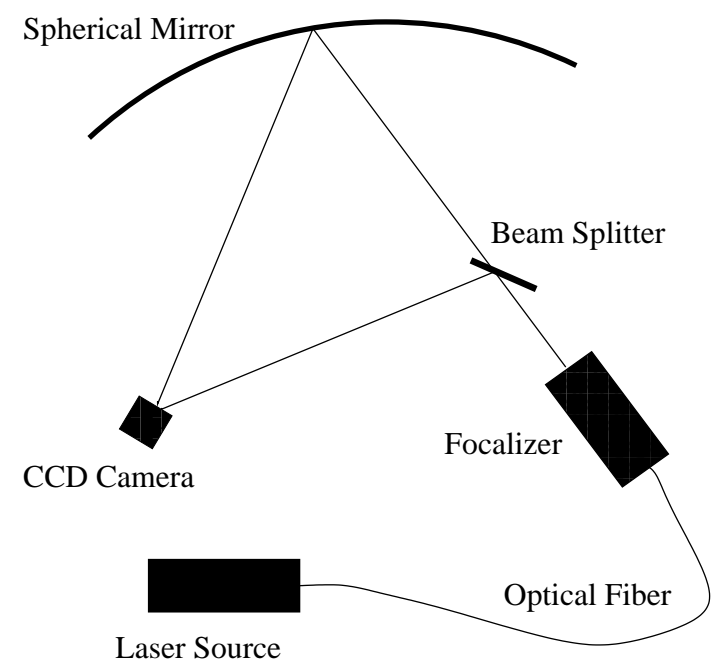

Figure 6.17: Schematic of the mirror alignment monitoring system.

\section{Mirror alignment}

Mechanical stability of the mirrors is of paramount importance to achieve the required Cherenkov angular resolution. This stability is challenging due to the size of the mirrors and the difficulty of securing them without introducing mechanical distortions to the reflecting surface. An alignment system which uses a CCD camera monitors mirror movements. On the focal plane of a given mirror, the images of two light spots are formed: the first comes directly from a laser source and the second is reflected back from the monitored mirror. Any change in the relative position of the two spots is an indication of mirror mechanical misalignment and the information is used to make an off-line correction. A schematic of the system is shown in figure 6.17. The laser light source feeds a bundle of optical fibers which generate all the required light paths (eight mirrors will be monitored in RICH 1, 16 in RICH 2). The beams are then directed to the beam splitters which guide the reference beams directly to the CCDs and the secondary beams to the mirrors.

It can be demonstrated that there is a linear transformation law between a change in the mirror tilt $\left(\Delta \Theta_{x}, \Delta \Theta_{y}\right)$ and the relative movement of the reflected spot on the $\operatorname{CCD}\left(\Delta_{x}, \Delta_{y}\right)$ :

$$
\begin{aligned}
\Delta \Theta_{x} & =A \Delta_{x}+B \Delta_{y} \\
\Delta_{y} & =C \Delta_{x}+D \Delta_{y},
\end{aligned}
$$

where the parameters $A$ to $D$ are fixed constants which depend on the geometry of the system. The mirror tilts can then be determined by inverting this transformation after observing the spot movement. The system tracks the displacement between the two beam spots, even if the spots move together, with an accuracy of better than $0.01 \mathrm{mrad}$.

\subsubsection{RICH performance}

\section{Test Beam studies}

An extensive test beam programme has allowed the RICH photodetectors, readout electronics and radiators to be evaluated with Cherenkov radiation in a realistic environment. Of particular impor- 
Table 6.2: Comparison of the measured and expected photon yields for the individual HPDs in the RICH test beam run. The first column indicates the label of each HPD.

\begin{tabular}{|l|c|c|c|}
\hline HPD & $\mu_{\text {meas. }}$ & $\mu_{\exp }$ & $\mu_{\text {meas. }} / \mu_{\text {exp }}$ \\
\hline \hline L0 & $8.1 \pm 0.10$ & $11.4 \pm 0.73$ & $0.71 \pm 0.07$ \\
\hline L1 & $10.2 \pm 0.16$ & $10.0 \pm 0.66$ & $1.02 \pm 0.07$ \\
\hline C1 & $11.5 \pm 0.33$ & $11.2 \pm 0.78$ & $1.02 \pm 0.07$ \\
\hline R0 & $8.7 \pm 0.24$ & $8.9 \pm 0.73$ & $0.98 \pm 0.09$ \\
\hline R1 & $10.1 \pm 0.03$ & $10.7 \pm 0.83$ & $0.95 \pm 0.08$ \\
\hline
\end{tabular}

tance has been the comparison of the expected and observed photoelectron yields and Cherenkov angle resolutions. Because the predictions of these quantities for test beam operation are made using a simulation with identical assumptions to those of the full LHCb MonteCarlo, agreement between prediction and observation is an important indicator that the RICH system will perform to specification.

A demonstrator RICH detector was operated in a test beam utilizing pre-production photon detectors and realistic prototypes of the associated RICH electronics [119]. The tests were performed at the CERN-PS in the T9 facility using $10 \mathrm{GeV} / c$ pions together with an $\mathrm{N}_{2}$ gas radiator to generate Cherenkov light. The HPDs were arranged in columns with the close-packing arrangement that will be used in the experiment and were read out at the LHC frequency of $40 \mathrm{MHz}$. In this configuration, the Cherenkov rings from the $N_{2}$ gas radiator were fully contained within a single HPD. Having included corrections for the asynchronous nature of the test beam set-up, the photoelectron yields were found to be in good agreement with those expected from the MonteCarlo simulation, as shown in table 6.2. A distribution of the number of pixel hits observed per event on a single HPD is shown in figure 6.18. Uncertainties in the corrections for the asynchronous beam structure explain the residual differences between the data and simulated distributions.

The distribution of reconstructed Cherenkov angles was also found to be in good agreement with the simulation (see figure 6.19). The Cherenkov angle resolution was determined to be $1.66 \pm 0.03 \mathrm{mrad}$, to be compared with the simulation expectation of $1.64 \mathrm{mrad}$. The dominant contributions to the Cherenkov angle resolution came from the uncertainty in the beam direction, the HPD point-spread function and the HPD pixelisation. The relative contributions of the latter two effects were significant owing to the test beam geometry but are not expected to be dominant in the final $\mathrm{LHCb}$ experiment.

\section{Detector simulation}

The LHCb RICH system is modelled as part of the LHCb Simulation program based on the GEANT4 simulation toolkit (see chapter 10). All important aspects of the geometry and the material description are fully simulated. A database is setup with all this information which is then input into the simulation and the reconstruction programs.

The Cherenkov light generation is performed inside GEANT4, and the photons propagated with full knowledge of the expected reflectivities, transmissions and refraction effects at the various optical elements. The Rayleigh scattering in aerogel and absorption in the various media are also 


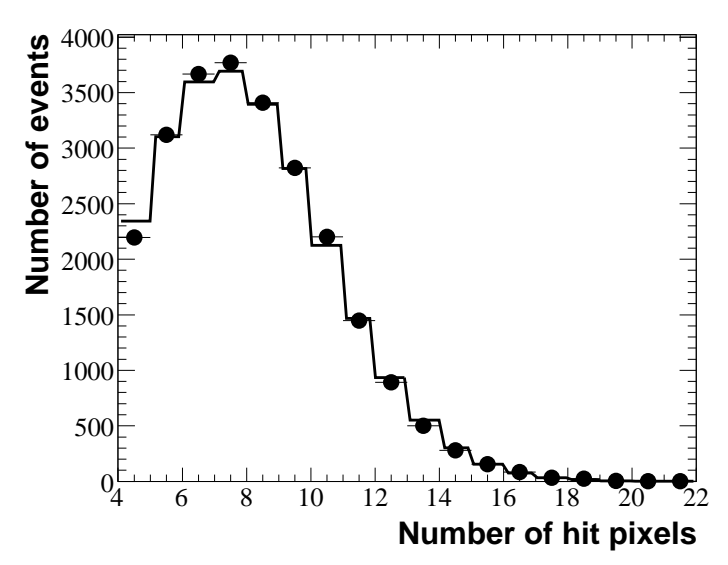

Figure 6.18: Number of pixel hits per event for data (points) and simulation (histogram) for a single HPD used in the RICH test beam run.

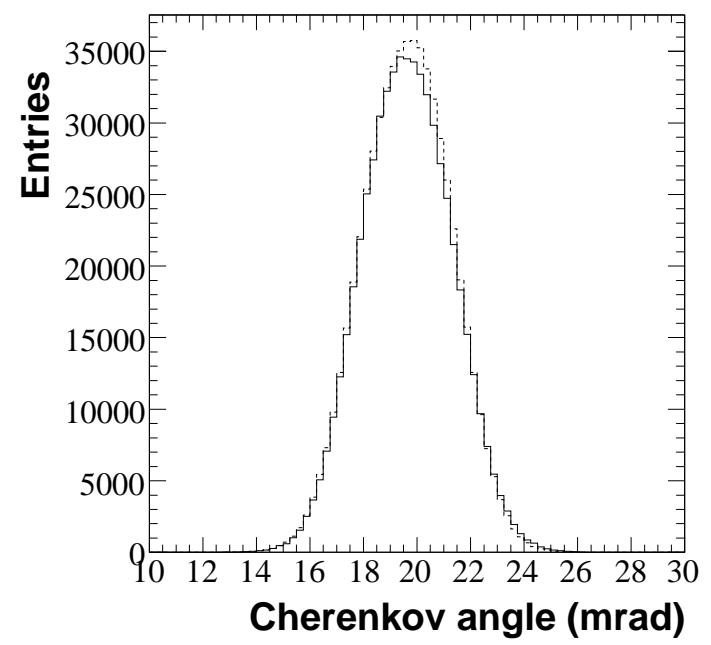

Figure 6.19: Reconstructed Cherenkov angle for data (solid histogram) and simulation (dotted line) on one of the HPDs used in the 2004 $\mathrm{RICH}$ test beam run.

included. Inside the HPDs, using the measured quantum efficiencies and cross-focussing relations, the photoelectrons created from the photocathode are mapped down onto the silicon detectors (hits location and energy). From these hits, the charge sharing in the pixels and the response of frontend readout are modelled in a separate package, which creates the digitized hits in the same format which will be output from the operational detector. All known sources of background are simulated. The most important components come from Rayleigh scattered photons in the aerogel, rings from secondary particles without associated reconstructed tracks and Cherenkov light generation in the HPD windows from traversing charged particles. More information on the LHCb RICH simulation may be found in [120]. Figure 6.20 shows a simulated event display in RICH 1.

For a given pixel-track association, the apparent Cherenkov angle is reconstructed through knowledge of the track direction, the hit pixel location, and the geometry of the RICH optics. This reconstruction assumes that the Cherenkov photon was emitted at the midpoint of the radiator. In simulation the resolution on the Cherenkov angle per photoelectron, and the mean number of photoelectrons detected per $\beta \approx 1$ track can be determined by using truth information to ensure that the hit pixel-track association is correct. The mean number of photoelectrons is found to be 6.7 for aerogel, 30.3 for $\mathrm{C}_{4} \mathrm{~F}_{10}$ and 21.9 for $\mathrm{CF}_{4}$. The resolution results are shown in table 6.3, where both the total resolutions and the individual contributions are listed. The single photoelectron resolution is largest for the aerogel, at $2.6 \mathrm{mrad}$, and smallest for the $\mathrm{CF}_{4}$, at $0.7 \mathrm{mrad}$. These resolutions receive contributions from the uncertainty associated with the photon emission point, the chromatic dispersion of the radiator, the finite pixel size and the point spread function (together listed as 'HPD' in the table). For the aerogel it is the chromatic dispersion error which dominates, whereas for the other two radiators the contributions are well matched. An additional uncertainty comes from the reconstruction of the track direction.

The resulting particle identification performance of the RICH system will be discussed in section 10 . 


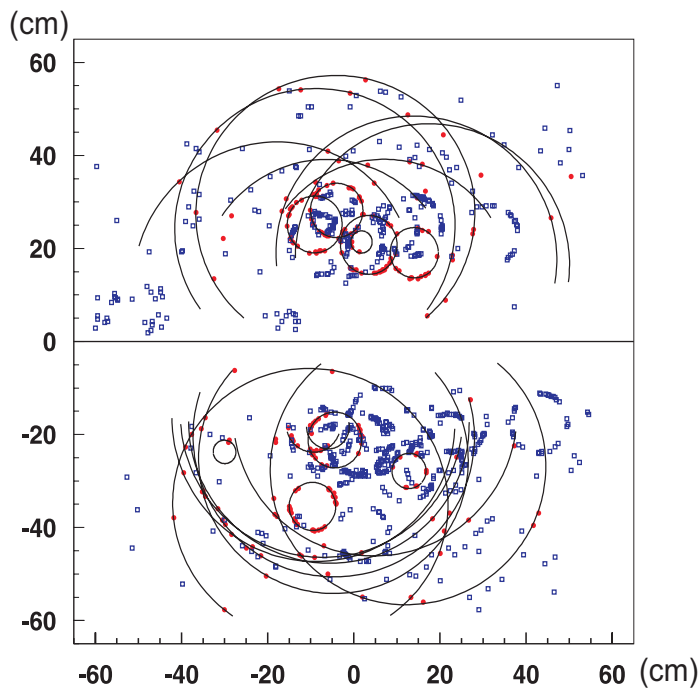

Figure 6.20: Display of a typical LHCb event in RICH 1.

Table 6.3: Single photoelectron resolutions for the three RICH radiators. All numbers are in mrad. Individual contributions from each source are given, together with the total.

\begin{tabular}{|l|ccc|}
\hline & Aerogel & $\mathrm{C}_{4} \mathrm{~F}_{10}$ & $\mathrm{CF}_{4}$ \\
\hline Emission & 0.4 & 0.8 & 0.2 \\
Chromatic & 2.1 & 0.9 & 0.5 \\
HPD & 0.5 & 0.6 & 0.2 \\
Track & 0.4 & 0.4 & 0.4 \\
\hline Total & 2.6 & 1.5 & 0.7 \\
\hline
\end{tabular}

\subsection{Calorimeters}

The calorimeter system performs several functions. It selects transverse energy hadron, electron and photon candidates for the first trigger level (L0), which makes a decision $4 \mu \mathrm{s}$ after the interaction. It provides the identification of electrons, photons and hadrons as well as the measurement of their energies and positions. The reconstruction with good accuracy of $\pi^{0}$ and prompt photons is essential for flavour tagging and for the study of B-meson decays and therefore is important for the physics program.

The set of constraints resulting from these functionalities defines the general structure and the main characteristics of the calorimeter system and its associated electronics [1, 121]. The ultimate performance for hadron and electron identification will be obtained at the offline analysis level. The requirement of a good background rejection and reasonable efficiency for B decays adds demanding conditions on the detector performance in terms of resolution and shower separation. 

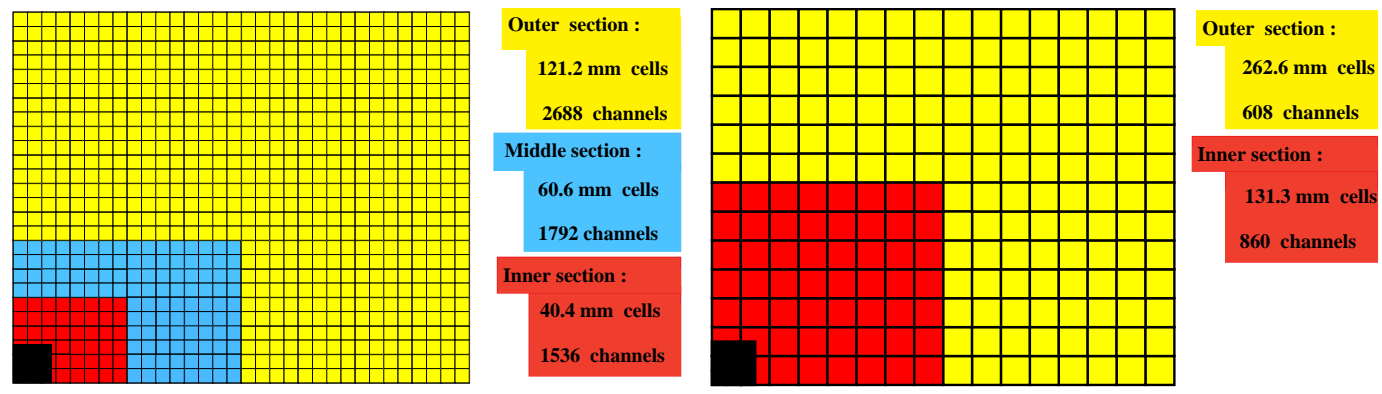

Figure 6.21: Lateral segmentation of the SPD/PS and ECAL (left) and the HCAL (right). One quarter of the detector front face is shown. In the left figure the cell dimensions are given for the ECAL.

\subsubsection{General detector structure}

A classical structure of an electromagnetic calorimeter (ECAL) followed by a hadron calorimeter (HCAL) has been adopted. The most demanding identification is that of electrons. Within the bandwidth allocated to the electron trigger (cf. section 7.1.2) the electron Level 0 trigger is required to reject $99 \%$ of the inelastic pp interactions while providing an enrichment factor of at least 15 in $\mathrm{b}$ events. This is accomplished through the selection of electrons of large transverse energy $E_{T}$. The rejection of a high background of charged pions requires longitudinal segmentation of the electromagnetic shower detection, i.e. a preshower detector (PS) followed by the main section of the ECAL. The choice of the lead thickness results from a compromise between trigger performance and ultimate energy resolution [122]. The electron trigger must also reject a background of $\pi^{0}$ 's with high $E_{T}$. Such rejection is provided by the introduction, in front of the PS, of a scintillator pad detector (SPD) plane used to select charged particles. A thin lead converter is placed between SPD and PS detectors. At Level 0, the background to the electron trigger will then be dominated by photon conversions in the upstream spectrometer material, which cannot be identified at this stage. Optimal energy resolution requires the full containment of the showers from high energy photons. For this reason, the thickness of ECAL was chosen to be 25 radiation lengths [123]. On the other hand, the trigger requirements on the HCAL resolution do not impose a stringent hadronic shower containment condition. Its thickness is therefore set to 5.6 interaction lengths [124] due to space limitations.

The PS/SPD, ECAL and HCAL adopt a variable lateral segmentation (shown in figure 6.21) since the hit density varies by two orders of magnitude over the calorimeter surface. A segmentation into three different sections has been chosen for the ECAL and projectively for the SPD/PS. Given the dimensions of the hadronic showers, the HCAL is segmented into two zones with larger cell sizes.

All calorimeters follow the same basic principle: scintillation light is transmitted to a PhotoMultiplier (PMT) by wavelength-shifting (WLS) fibres. The single fibres for the SPD/PS cells are read out using multianode photomultiplier tubes (MAPMT), while the fibre bunches in the ECAL and HCAL modules require individual phototubes. In order to have a constant $E_{T}$ scale the gain in the ECAL and HCAL phototubes is set in proportion to their distance from the beampipe. Since the light yield delivered by the HCAL module is a factor 30 less than that of the ECAL, the HCAL tubes operate at higher gain. 


\subsubsection{Electronics overview}

The basic structure is dictated by the need to handle the data for the Level 0 trigger as fast as possible. The front-end electronics and the PS/SPD photomultipliers are located at the detector periphery. The HCAL and ECAL phototubes are housed directly on the detector modules. The signals are shaped directly on the back of the photomultiplier for the PS/SPD or after $12 \mathrm{~m}$ and $16 \mathrm{~m}$ long cables for ECAL and HCAL respectively. They are then digitized in crates positioned on top of the detectors and the trigger circuits, hosted in the same crates, perform the clustering operations required by the trigger [125]. For each channel, the data, sampled at the bunch crossing rate of $40 \mathrm{MHz}$, are stored in a digital pipeline until the Level-0 trigger decision. In order to exploit the intrinsic calorimeter resolution over the full dynamic range, ECAL and HCAL signals are digitized by a 12-bit flash ADC [126]. Ten bits are enough for the preshower, and the SPD information is only one bit, a simple discriminator recording whether a cell has been hit or not $[127,128]$. An additional requirement is to reduce the tails of signals associated to the bunch crossing preceding the one being sampled. For ECAL and HCAL, this goal can be achieved at the percent level by suitable signal treatment within $25 \mathrm{~ns}$. In the case of the PS and SPD, the signal shape fluctuations require the longest possible signal integration time. They therefore use a different front-end design for the integrator, as described in section 6.2.7. Finally, in order not to degrade the resolution, the electronic noise must remain at the least significant bit level [129]. At the short shaping times being used, this requires careful design of the very front-end part.

\subsubsection{The pad/preshower detector}

The pad/preshower (SPD/PS) detector uses scintillator pad readout by WLS fibres that are coupled to MAPMT via clear plastic fibres. The choice of a 64 channel MAPMT allowed the design of a fast, multi-channel pad detector with an affordable cost per channel.

The SPD/PS detector consists of a $15 \mathrm{~mm}$ lead converter $2.5 \mathrm{X}_{0}$ thick, that is sandwiched between two almost identical planes of rectangular scintillator pads of high granularity with a total of 12032 detection channels (figure 6.22 left). The sensitive area of the detector is $7.6 \mathrm{~m}$ wide and $6.2 \mathrm{~m}$ high. Due to the projectivity requirements, all dimensions of the SPD plane are smaller than those of the PS by $\sim 0.45 \%$. The detector planes are divided vertically into two halves. Each can slide independently on horizontal rails to the left and right side in order to allow service and maintenance work. The distance along the beam axis between the centres of the PS and the SPD scintillator planes is $56 \mathrm{~mm}$. In order to achieve a one-to-one projective correspondence with the ECAL segmentation (described in section 6.2.4), each PS and SPD plane is subdivided into inner (3072 cells), middle (3584 cells) and outer (5376 cells) sections with approximately $4 \times 4,6 \times 6$ and $12 \times 12 \mathrm{~cm}^{2}$ cell dimensions.

The cells are packed in $\sim 48 \times 48 \mathrm{~cm}^{2}$ boxes (detector units) that are grouped into supermodules. Each supermodule has a width of $\approx 96 \mathrm{~cm}$, a height of $\approx 7.7 \mathrm{~m}$ and consists of detector units that form 2 rows and 13 columns. The space available for the SPD/PS detector between the first muon chamber and the electromagnetic calorimeter is only $180 \mathrm{~mm}$. Figure 6.22 (right) shows an individual scintillator pad with the WLS fibre layout. The diameter of the WLS fibre groove is a few mm smaller than the tile size; exact parameters of the tile geometry can be found in [130]. The basic plastic component is polystyrene to which primary and secondary WLS dopants, parater- 

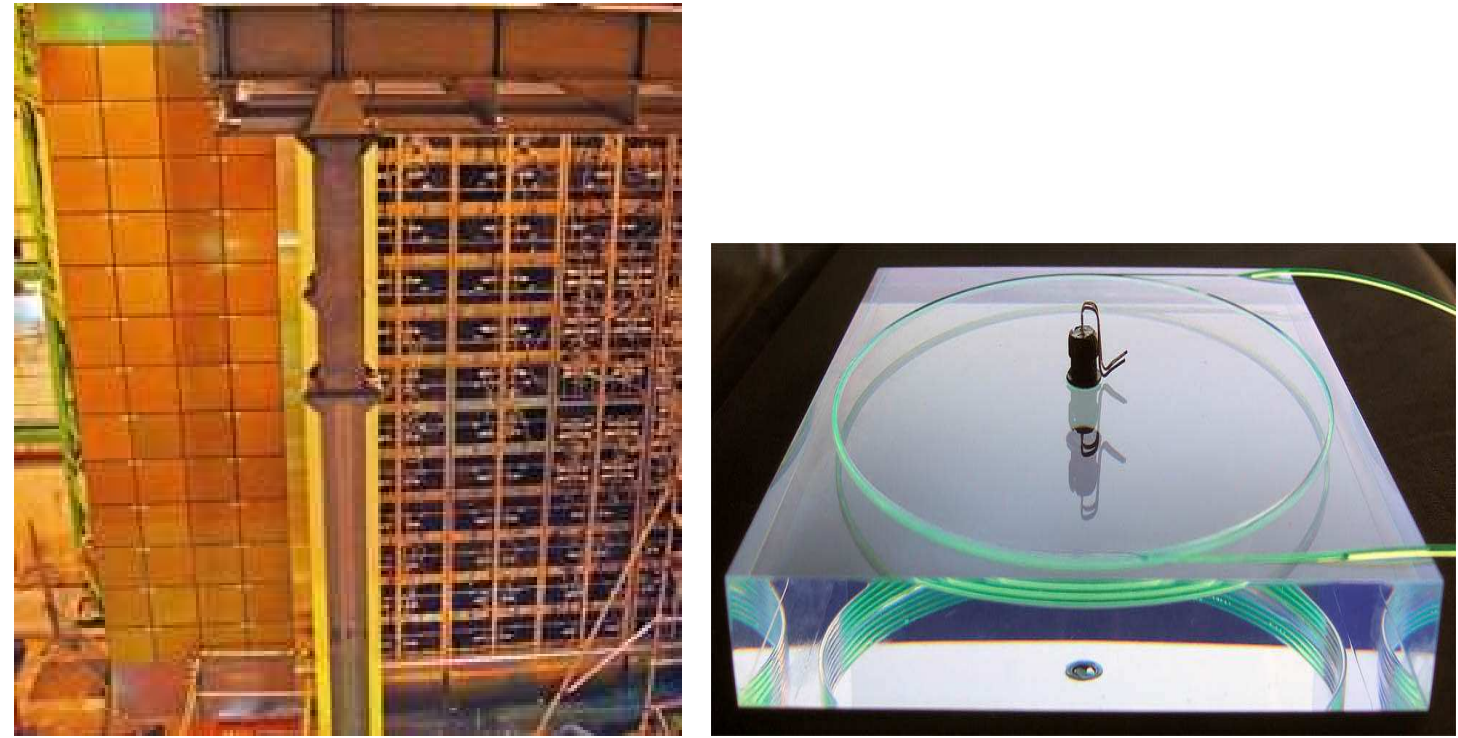

Figure 6.22: Front view of one half of the SPD/PS installed in the LHCb experimental hall (left). Individual scintillator pad with the WLS fibre layout and the LED housing in the middle (right).

phenyl (PTP), $1.5 \%$ and POPOP, $0.04 \%$, are added. ${ }^{11}$ The square structure of a pad is cut out from a $15 \mathrm{~mm}$ thick scintillator plate, and the scintillator surface is polished to reach the necessary optical quality. In order to maximize the light collection efficiency, WLS fibres are coiled and placed into a ring groove that is milled in the body of the cell. The rectangular cross section of the groove is $4.1 \mathrm{~mm}$ deep and $1.1 \mathrm{~mm}$ wide. The groove contains 3.5 loops of WLS fibre. The number of loops was chosen to achieve an overall optimization of the light collection efficiency [131] and the signal formation [132]. Two additional grooves are milled in the scintillator allowing both ends of the WLS fibre to exit the plate. The fibre is glued inside the groove ${ }^{12}$ using a dedicated semi-automatic device that provides the winding of the fibre and a uniform glue filling along the groove. A $1.0 \mathrm{~mm}$ diameter Y11(250) MS70 multi-cladding S-type WLS fibre ${ }^{13}$ was chosen as a reasonable compromise between light output and durability. The pad is wrapped with $0.15 \mathrm{~mm}$ thick TYVEK ${ }^{14}$ paper in order to improve the light reflection and to minimize the dead space between adjacent pads. Light produced by an ionizing particle in the scintillator is guided by the WLS fibre to the exit of the detector box. At this point optical connectors (described in [130]) join the WLS fibres to long clear fibres. The two clear fibres connected to the two ends of the WLS fibre of a given pad are viewed by a single MAPMT pixel [130]. The length of clear fibres varies from 0.7 to $3.5 \mathrm{~m}$ but all the fibres connected to a particular PMT have the same length in accordance with the front-end electronics specification $[127,128]$. The clear fibre allows the transport of the scintillator light from the SPD/PS planes over a few metres to the multi-anode PMT without significant attenuation.

The scintillator cells are grouped into self-supporting detector units that are packed inside square boxes with dimension $476 \mathrm{~mm} \times 476 \mathrm{~mm}(\mathrm{SPD})$ and $478 \mathrm{~mm} \times 478 \mathrm{~mm}$ (PS) boxes, yield-

\footnotetext{
${ }^{11}$ produced at SSI Institute for Single Crystals NAS of Ukraine, 60 Lenin Ave, Kharkov, 61001, Ukraine.

${ }^{12}$ with BICRON BC-600 glue, BICRON Corp., 12345 Kinsman Rd. Newbury OH 440 USA.

${ }^{13}$ KURARAY Corp., 3-10, Nihonbashi, 2 chome, Chuo-ku, Tokyo, Japan.

${ }^{14}$ TYVEK of type 1057D used, product of E.I. du Pont de Nemours and Company.
} 

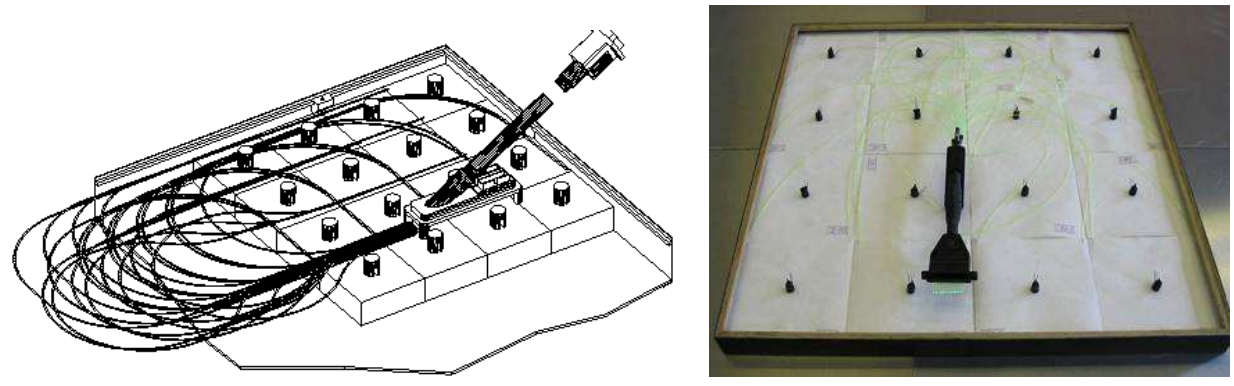

Figure 6.23: Fibre routing for the inner (left) and outer (right) module boxes.

ing a total of 26 boxes per supermodule. Since there are three sections with different cell sizes for the SPD/PS planes, the boxes are filled with a different number of pads with sizes that add up to $119 \mathrm{~mm}$ for the SPD to $119.5 \mathrm{~mm}$ for the PS planes. A dedicated fibreglass technology of box manufacturing was developed in order to obtain stiff boxes with a minimum amount of material between the adjacent cells of neighbouring boxes. The lateral walls of the monolithic box frame are manufactured using Fibreglass Reinforced Plastic (FGRP) with a wall thickness of $0.3 \mathrm{~mm}$. This fabrication method consists of a one cycle polymerization process with predefined curing parameters. To ensure light tightness a layer of black paper was incorporated in addition to the FGRP layer. The top and bottom layers of the box are made of $2 \mathrm{~mm}$ thick Al plate and G10 plastic. The bottom cover is glued to the FGRP frame by epoxy and the top one is fixed by screws. On the top cover there are output plastic ports, made of relatively cheap pressure die casting technology, disposed to allow exit of fibre bundles out of the box. The module assembly procedure is as follows. All cells in a detector unit are grouped in matrices of $4 \times 4$ cells. The scintillator pads with glued WLS fibres are placed into the box after quality control tests. The fibre ends are grouped by 32 , fit into a light-tight flexible tube and glued into an optical connector, before being cut and polished. The routing of fibres inside a box from one matrix is shown in figure 6.23. The bending diameter always exceeds $100 \mathrm{~mm}$. Depending on the number of scintillator cells inside a unit, the boxes are equipped with one (outer region), four (middle region) or nine (inner region) output port(s) and light connector(s).

The detector units are designed to be mounted on a supermodule support plate. All supermodules of the SPD/PS planes have identical design. Each consists of 26 detector units mounted on a long aluminium strip in two columns. The photomultiplier tubes are located on both the top and bottom ends of the supermodule support outside the detector acceptance. The detector units are optically connected to the PMTs by bundles of 32 clear fibres, enclosed in a light-tight plastic tube, by means of a photo-tube coupler. The PMT is a (8-stage) R5900-M64 manufactured by Hamamatsu ${ }^{15}[133,134]$ and has a bialkali photocatode segmented into 64 pixels of $2 \times 2 \mathrm{~mm}^{2}$. The HV is provided by a Cockroft-Walton voltage multiplier.

The quality of the phototubes has been extensively studied using measurements of the nonuniformities of anode response within one MAPMT, the linearity over the required dynamic range of the PS, the absolute gain of the MAPMT channels and the electronic crosstalk. The nonuniformity

\footnotetext{
${ }^{15}$ HAMAMATSU Photonics KK, Electron Tube Center, 314-5, Shimokanzo, Toyooka-village, Iwata-gun, Shizuokaken, 438-01 Japan.
} 
of response within one MAPMT was found to be in a ratio of 1 to 2 (minimum to maximum) for most of the installed tubes. A special care has been brought to the selection of the best tubes for the PS in order to limit the digital correction to at most a factor of 2 at the level of the front-end (FE) electronics. While the crosstalk between the PMT electronics channels has been measured to be negligible, a large number of tubes showed large crosstalk produced at the level of the focussing grid, immediately behind the MAPMT photocathode. This was one of the major cause for rejection. The response linearity was found to be well within specification for all the tested phototubes [135].

Specific measurements of the MAPMT behaviour in the magnetic field were carried out as well. It has been discovered that these phototubes, initially thought to be robust in a magnetic field were significantly sensitive to fields as weak as $1 \mathrm{mT}$. A dedicated magnetic shielding has been designed. A MuMetal cylinder ( $6 \mathrm{~cm}$ long with a $4 \mathrm{~cm}$ diameter) is used along the tube axis and the MAPMT (together with the very front-end (VFE) electronics) are housed in a box made of soft iron. Special attention was given to evaluate the long term behaviour of the phototubes subject to the conditions of the largest illumination of the PS (DC currents for the SPD are much smaller than for the PS [136]). The PMT gain is set to $10^{4}$, which gives stable conditions of operation. Prior to the installation all the SPD and PS modules were tested using a cosmic ray facility. Using a reference LED the average number of photoelectrons per Minimum Ionizing Particle (MIP) was measured to be 26, 28 and 21 for the PS and SPD cells of the inner, middle and outer regions correspondingly. During the production phase the light yield was measured for all tiles using a gamma radiation source. The tiles with similar light yield were grouped within the same matrix. The uniformity of matrices, also affected by the uniformity of the optical cables, was measured in a cosmic test of the detector supermodules. The uniformity of response within matrices was found to be within $6 \%$ RMS.

\section{Performance of the SPD/PS modules}

The $e / \pi$ separation performance of the PS prototype was measured in the $\mathrm{X} 7$ test beam at the CERN SPS with electrons and pions between 10 and $50 \mathrm{GeV} / \mathrm{c}$ momentum. The energy deposited in the PS for $50 \mathrm{GeV} / \mathrm{c}$ electrons and pions is shown in figure 6.24.

The measurements show that with a threshold of 4 MIPs (about 100 ADC channels), pion rejection factors of $99.6 \%, 99.6 \%$ and $99.7 \%$ with electron retentions of $91 \%, 92 \%$ and $97 \%$ are achieved for 10, 20 and $50 \mathrm{GeV} / \mathrm{c}$ particle momentum, respectively. From measurements with $20 \mathrm{GeV}$ electrons in the combined PS and ECAL test, it is confirmed that the energy resolution of the ECAL does not deteriorate if one corrects for the energy absorbed in the PS lead converter using the energy measured in the PS scintillator [131]. In order to separate photons and electrons at Level 0 of the ECAL trigger, the information from the SPD positioned in front of the lead absorber is used. Charged particles deposit energy in the scintillator material, while neutrals particles do not interact. However, several processes can lead to an energy deposit in the scintillator and result in the misidentification of photons. The dominant one is the photon conversion in the detector material before the SPD (see sections 10.2.3 and 10.2.4). Two other sources are interactions in the SPD that produce charged particles inside the SPD, and backwards moving charged particles, back splash, that are generated in the lead absorber or in the electromagnetic calorimeter. The latter two 


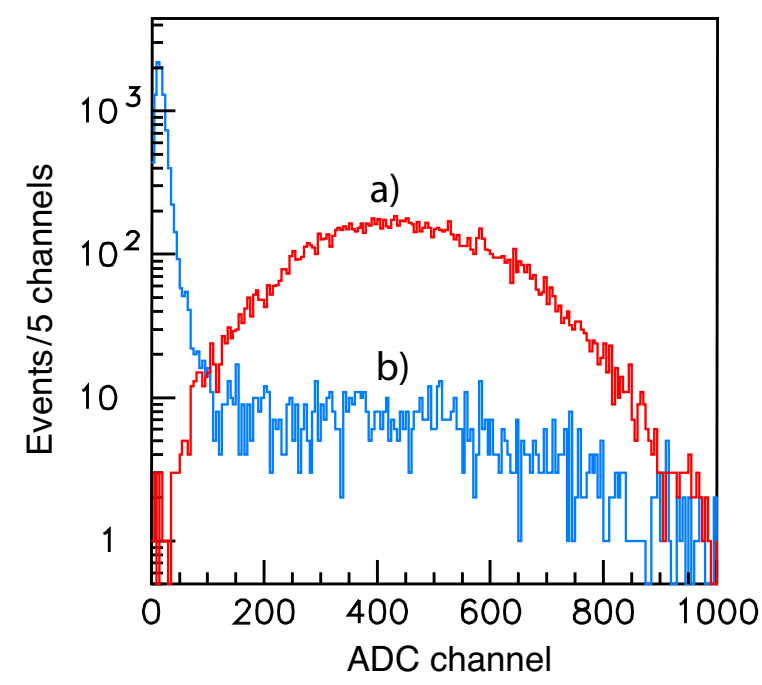

Figure 6.24: Energy deposition of (a) $50 \mathrm{GeV}$ electrons and (b) pions in the PS.
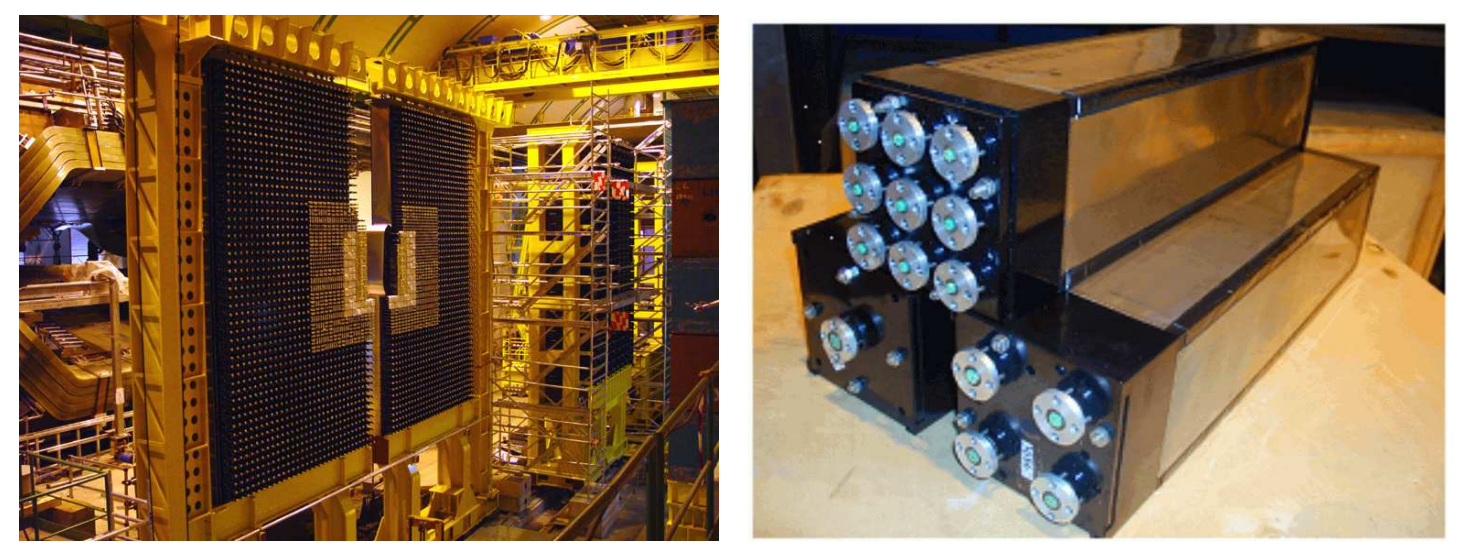

Figure 6.25: Downstream view of the ECAL installed (but not completely closed) with the exception of some detector elements above the beam line. Outer, middle and inner type ECAL modules (right).

effects have been studied with the prototype in both a tagged photon beam and beams of electrons and pions of different energies, in the CERN X7 test beam area. The results were compared with simulation. The measurements for photon energies between 20 and $50 \mathrm{GeV}$ show [137] that the probability of photon misidentification due to interactions in the SPD scintillator is $(0.8 \pm 0.3) \%$, when applying a threshold of 0.7 MIPs. The probability to pass this threshold due to backward moving charged particles was measured to be $(0.9 \pm 0.6) \%$ and $(1.4 \pm 0.6) \%$ for 20 and $50 \mathrm{GeV}$ photons, respectively. All these numbers are in very good agreement with MonteCarlo simulation study. More details on backsplash study can be found in [137]. 
Table 6.4: Main parameters of the LHCb electromagnetic calorimeter. (*) Only 1536 channels are active, instead of 1584 , due to the clearance around the beam.

\begin{tabular}{|l|c|c|c|}
\hline & Inner section & Middle section & Outer section \\
\hline Inner dimension, $x \times y, \mathrm{~cm}^{2}$ & $65 \times 65$ & $194 \times 145$ & $388 \times 242$ \\
\hline Outer dimension, $x \times y, \mathrm{~cm}^{2}$ & $194 \times 145$ & $388 \times 242$ & $776 \times 630$ \\
\hline Cell size, $\mathrm{cm}^{2}$ & $4.04 \times 4.04$ & $6.06 \times 6.06$ & $12.12 \times 12.12$ \\
\hline \# of modules & 176 & 448 & 2688 \\
\hline \# of channels & $1536^{*}$ & 1792 & 2688 \\
\hline \# of cells per module & 9 & 4 & 1 \\
\hline \# of fibres per module & 144 & 144 & 64 \\
\hline Fibre density, $\mathrm{cm}^{-2}$ & 0.98 & 0.98 & 0.44 \\
\hline
\end{tabular}

\subsubsection{The electromagnetic calorimeter}

The shashlik calorimeter technology, i.e. a sampling scintillator/lead structure readout by plastic WLS fibres, has been chosen for the electromagnetic calorimeter not only by LHCb but by a number of other experiments [138, 139]. This decision was made taking into account modest energy resolution, fast time response, acceptable radiation resistance and reliability of the shashlik technology, as well as the experience accumulated by other experiments [140-142]. Specific features of the LHCb shashlik ECAL are an improved uniformity and an advanced monitoring system. The design energy resolution of $\sigma_{E} / E=10 \% / \sqrt{E} \oplus 1 \%(\mathrm{E}$ in $\mathrm{GeV})$ results in a B mass resolution of $65 \mathrm{MeV} / \mathrm{c}^{2}$ for the $B \rightarrow K^{*} \gamma$ penguin decay with a high- $E_{T}$ photon and of $75 \mathrm{MeV} / \mathrm{c}^{2}$ for $B \rightarrow \rho \pi$ decay with the $\pi^{0}$ mass resolution of $\sim 8 \mathrm{MeV} / \mathrm{c}^{2}$.

The electromagnetic calorimeter, shown in figure 6.25 (left), is placed at $12.5 \mathrm{~m}$ from the interaction point. The outer dimensions of the ECAL match projectively those of the tracking system, $\theta_{x}<300 \mathrm{mrad}$ and $\theta_{y}<250 \mathrm{mrad}$; the inner acceptance is mainly limited by $\theta_{x, y}>25 \mathrm{mrad}$ around the beampipe due to the substantial radiation dose level. The hit density is a steep function of the distance from the beampipe, and varies over the active calorimeter surface by two orders of magnitude. The calorimeter is therefore subdivided into inner, middle and outer sections (table 6.4) with appropriate cell size, as shown in figure 6.25 (right).

A module is built from alternating layers of $2 \mathrm{~mm}$ thick lead, $120 \mu \mathrm{m}$ thick, white, reflecting TYVEK $^{16}$ paper and $4 \mathrm{~mm}$ thick scintillator tiles. In depth, the $66 \mathrm{~Pb}$ and scintillator layers form a $42 \mathrm{~cm}$ stack corresponding to $25 \mathrm{X}_{0}$. The Moliere radius of the stack is $3.5 \mathrm{~cm}$. The stack is wrapped with black paper, to ensure light tightness, pressed and fixed from the sides by the welding of $100 \mu \mathrm{m}$ steel foil.

The scintillator tiles are produced from polystyrene ${ }^{17}$ with $2.5 \% \mathrm{PTP}^{18}$ and $0.01 \% \mathrm{POPOP}^{19}$ admixtures. The scintillator tile production employs a high pressure injection moulding technique. Tile edges are chemically treated to provide diffusive reflection and consequently improved light

\footnotetext{
${ }^{16}$ TYVEK of type 1025D used, product of E.I. du Pont de Nemours and Company.

${ }^{17}$ Polystherene in pellets, Polystyrol 165H, [Cn Hn], product of BASF AG, Badische Anilin- \& Soda Fabrik Aktiengesellschaft, Carl-Bosch-Strasse 38, D-67056 Ludwigshafen, Germany, mailto:global.info@basf.com.

${ }^{18}$ PTP, p-Terphenyl, 1,4-Diphenylbenzene, [C6 H5 C6 H4 C6 H5], product of FLUKA(TM), Sigma-Aldrich Chemie GmbH, CH-9470, Buchs, Switzerland, mailto:fluka@ sial.com.

${ }^{19}$ POPOP, 1,4-Bis(5-phenyl-2-oxazolyl)benzene, [C24 H16 N2 O2], product of FLUKA(TM), Sigma-Aldrich Chemie GmbH, CH-9470, Buchs, Switzerland, mailto:fluka@ sial.com.
} 

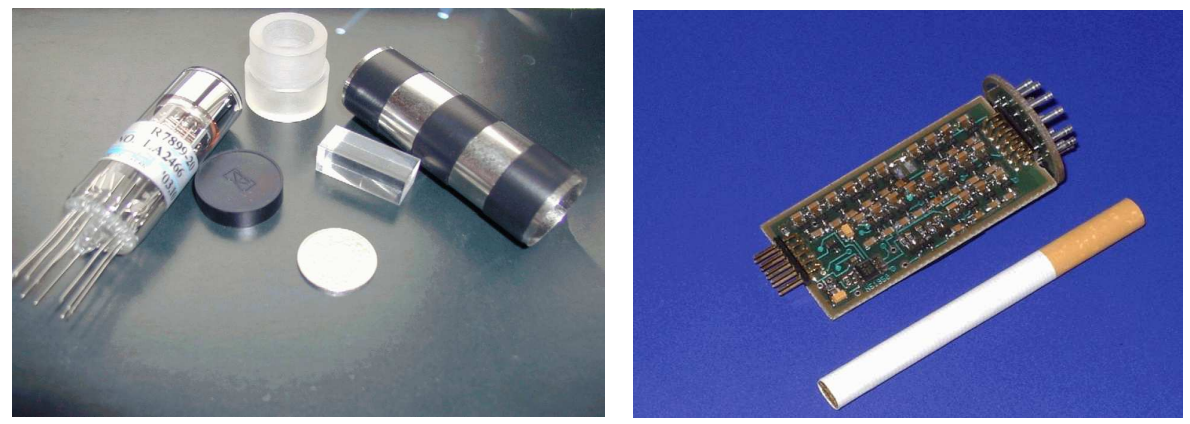

Figure 6.26: Hamamatsu R7899-20 phototube, light mixer and MuMetal magnetic shielding screen (left). Cockcroft-Walton voltage multiplier (right).

collection efficiency and prevent tile-to-tile light crosstalk for the inner and middle modules. The tile-to-tile light yield fluctuation has an RMS smaller than $2.5 \%$. The $\mathrm{Pb}$ plates are produced using sheet-metal stamping. In order to ease handling of the lead plates during module assembly the plates are covered with a $3 \mu \mathrm{m}$ thick layer of tin. Both the scintillator tiles and lead plates incorporate a pattern of precisely positioned holes that are needed for the traversing fibres. The demanding tolerances are ensured by injection moulding for the scintillator tiles and a punching technique for the lead plates.

The light from the scintillator tiles is absorbed, re-emitted and transported by $1.2 \mathrm{~mm}$ diameter WLS Kuraray Y-11(250)MSJ fibres, traversing the entire module. In order to improve light collection efficiency the fibres are looped such that each traverses the module twice, the looped part remaining in a housing outside the front of the module. Fibre bending under uniformly distributed dry heat made it possible to produce fibre loops with radii as small as $10 \mathrm{~mm}$, where the light loss is determined by the geometrical optics of reflection down the loop. When measured after the fibre loop, the light yield varies from loop to loop with an RMS of the spread of $1.6 \%$. The fibres belonging to each cell are bundled at the rear end of the module and polished.

The light is read out with Hamamatsu R7899-20 phototubes where the high voltage is provided by a Cockcroft-Walton (CW) base (figure 6.26).

Before installation all ECAL modules underwent detailed quality control at various stages of production. The final control was made with cosmic rays [143]. The standard deviations of the mean energy deposition by a MIP in the ECAL module was measured to be $7.4 \%, 4.4 \%$ and $6.0 \%$ for the outer, middle and inner sections respectively. The number of photoelectrons recorded for a MIP and for electrons in the test beam was calculated using the measurements of the amplitude and width of the LED signal. This method gives for the inner modules about 3100 photoelectrons per $\mathrm{GeV}$ of deposited energy, 3500 for the middle and 2600 for the outer modules. The differences are due to the differing WLS fibre density in the inner, middle and outer modules and to the cell size differences which influence the probability of photon absorption in the scintillating tiles.

\section{Performance of the ECAL modules}

The energy resolution and, in particular, the uniformity in response of the ECAL were studied at the test beam [144]. Only a few percent of the scintillation light is registered by the phototubes 

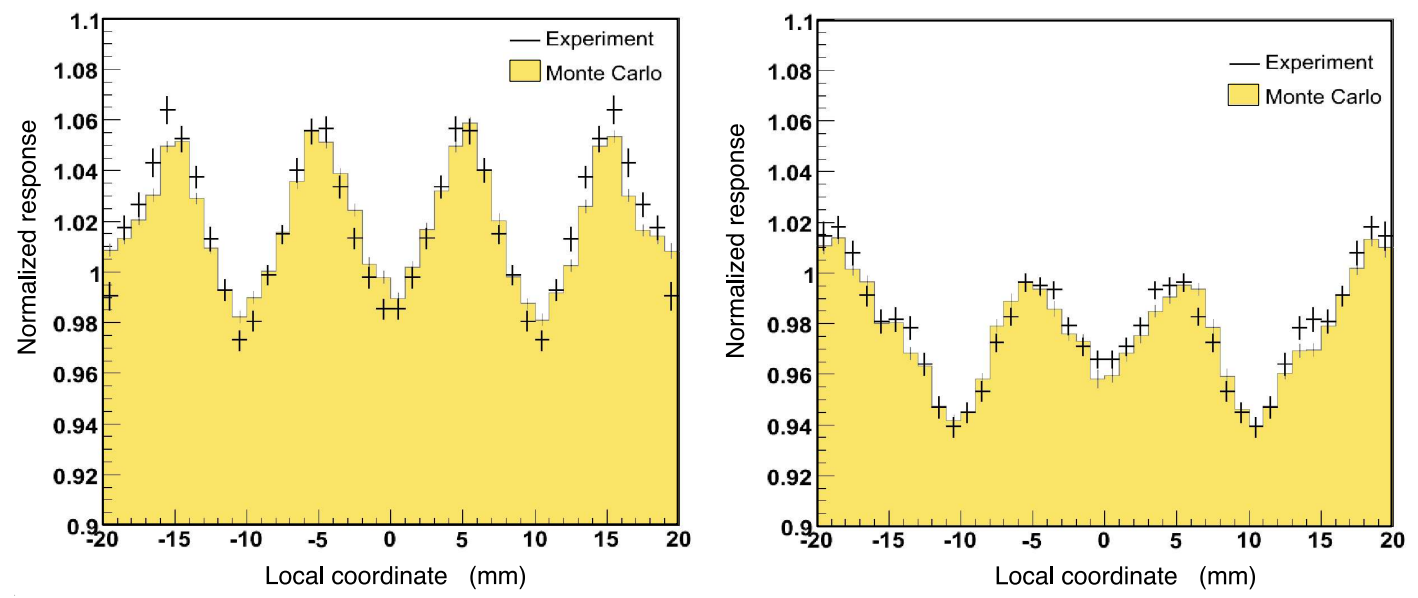

Figure 6.27: Uniformity of response to $100 \mathrm{GeV} / \mathrm{c}^{2}$ muons of the inner module. The scan was made in $1 \mathrm{~mm}$ wide slices through the fibre positions (left) and in between two fibre rows (right).

after capture and re-emission in the WLS fibres; about half of this light is delivered by the WLS fibres close to the light's origin. Some lateral nonuniformity in the light collection efficiency was expected from two sources: imperfect reflection from tile edges (the so-called global nonuniformity effect), and a dependence on the emission point of the scintillating light with respect to the fibres (local or inter-fibre nonuniformity). The global nonuniformity depends on the mean light path, which is a function of tile transparency, edge reflection quality and fibre density. In addition the module response at the edge is further degraded due to the presence of the stainless steel foils between the active volumes of two adjacent modules. The local nonuniformity is affected by the inter-fibre distance and the diameter of the fibre.

The light collection efficiency was studied using test beam data and a dedicated MonteCarlo simulation of the light propagation and absorption in the plastic tiles which takes into account the transparency of the scintillator as a function of wavelength, the tile-edge and TYVEK reflection properties and the WLS fibre absorption spectrum. The modules of the inner, middle and outer sections were scanned using $100 \mathrm{GeV} / \mathrm{c}$ muons with a $1 \mathrm{~mm}$ transverse step. As shown in figure 6.27, the measured inner module response is well described by MonteCarlo simulation. Shown are two different scans for the inner module, one using $1 \mathrm{~mm}$ wide slices through the WLS fibre positions (left plot), and the other between two fibre rows (right plot). In the outer modules the inter-fibre nonuniformity is slightly higher due to a factor of 1.5 larger fibre-to-fibre distance (15 $\mathrm{mm}$ instead of $10 \mathrm{~mm}$ for the inner and middle modules). The global nonuniformity of response was measured to be negligible for all module types. A slight increase of response in the vicinity of the module edges is explained by diffuse reflection from the boundary layer introduced by the dedicated chemical treatment of the tile edges. The effect of signal loss in the dead material between the modules is overcompensated by $8 \%$ at -20 and $20 \mathrm{~mm}$, as visible in figure 6.27 (right). Electromagnetic showers of electrons and photons considerably reduce the inter-fibre nonuniformity of response compared to that measured for MIPs. The results of a lateral scan with $50 \mathrm{GeV} / \mathrm{c}$ electrons is shown in figure 6.28 for the inner and outer ECAL modules. The module response is 

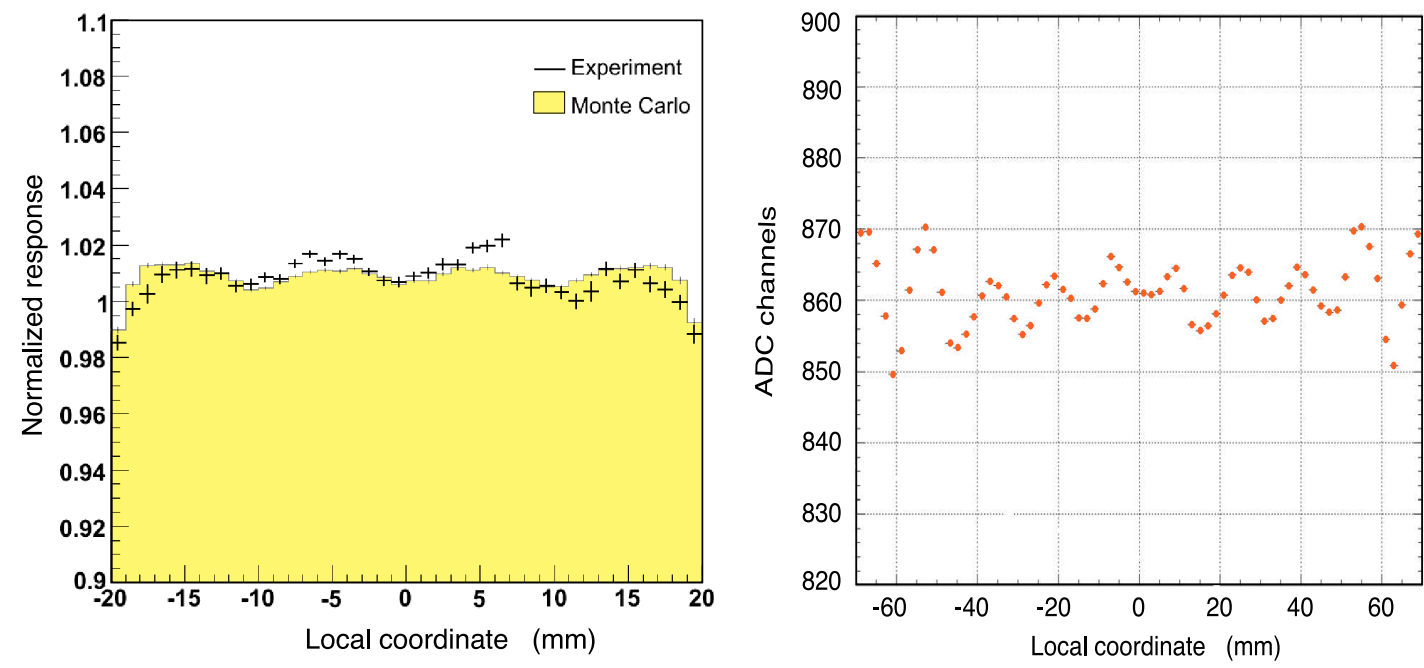

Figure 6.28: Uniformity of response to $50 \mathrm{GeV} / \mathrm{c}$ electrons of the inner (left) and outer (right) modules. The scan was made in $1 \mathrm{~mm}$ wide slices through the fibre positions.

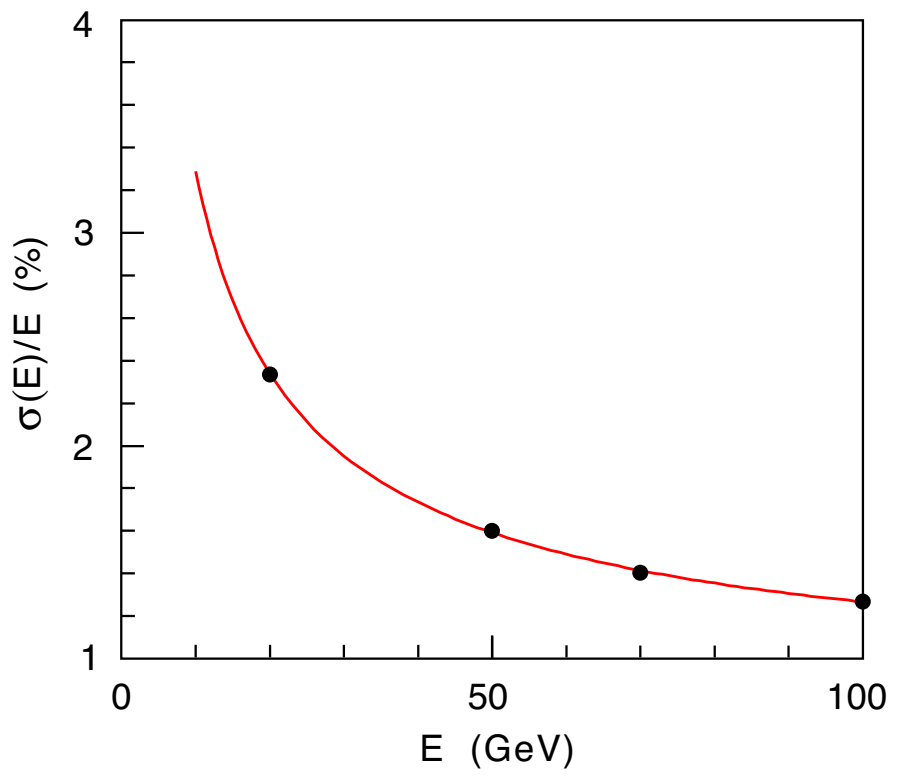

Figure 6.29: The energy resolution as measured with electrons over a surface of $( \pm 15 \mathrm{~mm}, \pm$ $30 \mathrm{~mm}$ ) in an outer module.

uniform within $0.8 \%$. The energy resolution of the ECAL modules has been determined at the test beam. The parametrization $\sigma_{E} / E=\mathrm{a} / \sqrt{E} \oplus \mathrm{b} \oplus \mathrm{c} / E(\mathrm{E}$ in $\mathrm{GeV})$ is used, where $\mathrm{a}, \mathrm{b}$ and $\mathrm{c}$ stand for the stochastic, constant and noise terms respectively. Depending on the type of module and test beam conditions the stochastic and constant terms were measured to be $8.5 \%<\mathrm{a}<9.5 \%$ and $\mathrm{b}$ $\sim 0.8 \%$ (see figure 6.29 ). 

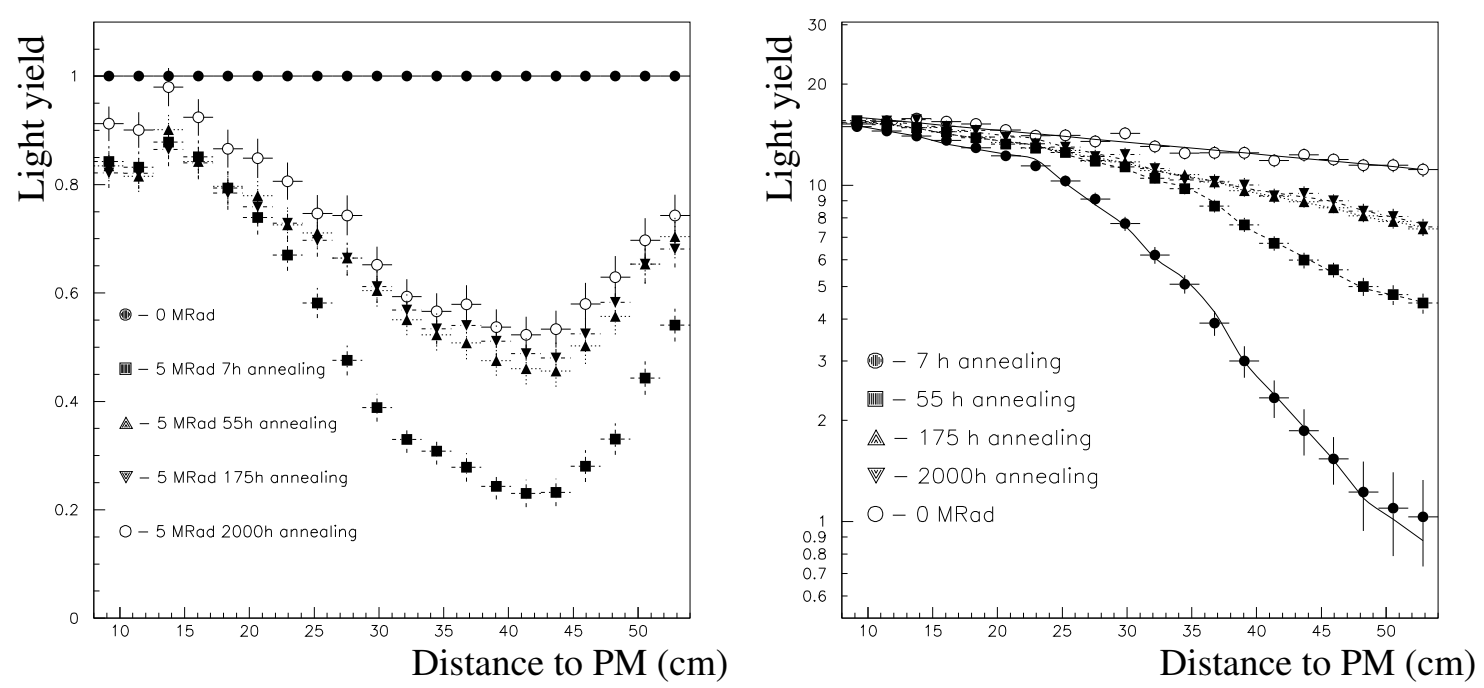

Figure 6.30: Scintillator (left) and WLS fibre (right) degradation and annealing effect after irradiation at LIL. The light yield (scintillator) and PMT current (fibres) are shown vs. the distance to the PMT (the shower maximum position corresponds to a distance from the PMT of $42 \mathrm{~cm}$ ).

\section{Radiation resistance of the ECAL modules}

Detailed measurements and simulation studies were performed to determine the degradation of the ECAL resolution due to radiation damage of the optical components. The expected annual radiation dose at the shower maximum for the ECAL modules closest to the beampipe is $0.25 \mathrm{MRad}$ assuming a luminosity of $2 \times 10^{32} \mathrm{~cm}^{-2} \mathrm{~s}^{-1}$ and $10^{7} \mathrm{~s}$ per nominal year. The optical components of the ECAL modules, namely the $40 \times 40 \mathrm{~mm}^{2}$ scintillating tiles and WLS fibres, were irradiated at the LEP Injector Linac (LIL) at CERN with electrons of $500 \mathrm{MeV}$ energy. The total irradiation dose was as large as $5 \mathrm{MRad}$ at a dose delivery rate of $10 \mathrm{Rad} / \mathrm{s}$; that is 200 times higher than the rate expected at $\mathrm{LHCb}$. Figure 6.30 shows the degradation in light yield and transparency, and the subsequent annealing of the irradiated components as a function of the distance to the phototube. In order to determine the degradation of the energy resolution due to irradiation, the energy response of the ECAL module was simulated using the expected longitudinal dose profile and the measured degradation of the scintillating tiles and WLS fibres in accordance with figure 6.30. The effects induced by irradiation of $2.2 \mathrm{MRad}$ leads to an increase of the constant term from $0.8 \%$ up to $1.5 \%$. Such a degradation in the ECAL resolution is expected after about eight years of operation under nominal conditions and is acceptable. However, taking into account simulation uncertainties on the expected radiation doses at the LHC, the ECAL detector was designed such that the modules closest to the beampipe could be replaced if this should become necessary.

\subsubsection{The hadron calorimeter}

The LHCb hadron calorimeter (HCAL) $[1,121]$ is a sampling device made from iron and scintillating tiles, as absorber and active material respectively. The special feature of this sampling structure is the orientation of the scintillating tiles that run parallel to the beam axis. In the lateral direction 

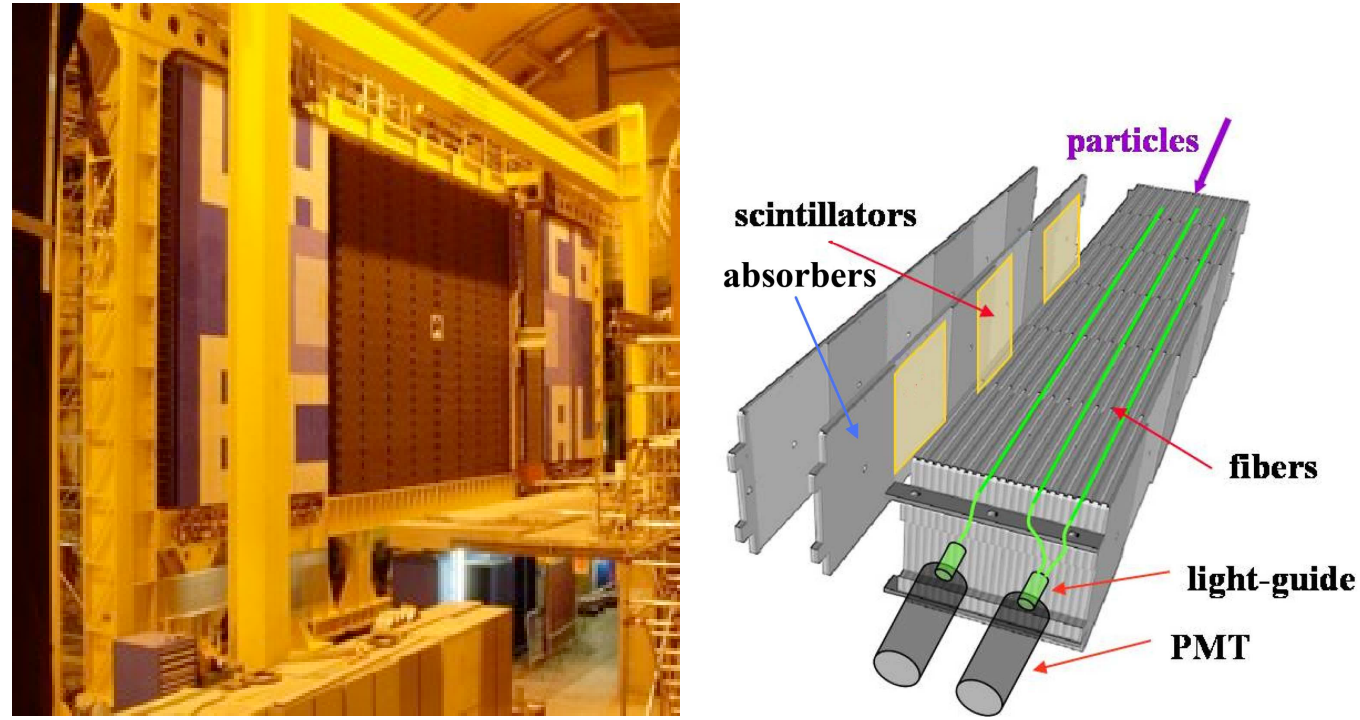

Figure 6.31: View from upstream of the HCAL detector installed behind the two retracted ECAL halves in the LHCb cavern (left). A schematic of the internal cell structure (right). The exploded view of two scintillator-absorber layers illustrates the elementary periodic structure of a HCAL module.

tiles are interspersed with $1 \mathrm{~cm}$ of iron, whereas in the longitudinal direction the length of tiles and iron spacers corresponds to the hadron interaction length $\lambda_{I}$ in steel. The light in this structure is collected by WLS fibres running along the detector towards the back side where photomultiplier tubes (PMTs) are housed. As shown in figure 6.31, three scintillator tiles arranged in depth are in optical contact with $1.2 \mathrm{~mm}$ diameter Kuraray ${ }^{20}$ Y-11(250)MSJ fibre [145] that run along the tile edges. The total weight of the HCAL is about 500 tons.

The HCAL is segmented transeversely [146] into square cells of size $131.3 \mathrm{~mm}$ (inner section) and $262.6 \mathrm{~mm}$ (outer section). Readout cells of different sizes are defined by grouping together different sets of fibres onto one photomultiplier tube that is fixed to the rear of the sampling structure. The lateral dimensions of the two sections are $\pm 2101 \mathrm{~mm}$ and $\pm 4202 \mathrm{~mm}$ in $x$ and $\pm 1838 \mathrm{~mm}$ and $\pm 3414 \mathrm{~mm}$ in $y$ for the inner and outer section, respectively. The optics is designed such that the two different cell sizes can be realized with an absorber structure that is identical over the whole HCAL. The overall HCAL structure is built as a wall, positioned at a distance from the interaction point of $z=13.33 \mathrm{~m}$ with dimensions of $8.4 \mathrm{~m}$ in height, $6.8 \mathrm{~m}$ in width and $1.65 \mathrm{~m}$ in depth. The structure is divided vertically into two symmetric parts that are positioned on movable platforms, to allow access to the detector. Each half is built from 26 modules piled on top of each other in the final installation phase. The assembled HCAL is shown in figure 6.31(left). The absorber structure, shown in figure 6.31 (right), is made from laminated steel plates of only six different dimensions that are glued together. Identical periods of $20 \mathrm{~mm}$ thickness are repeated 216 times in the module. One period consists of two $6 \mathrm{~mm}$ thick master plates with a length of $1283 \mathrm{~mm}$ and a height of $260 \mathrm{~mm}$ that are glued in two layers to several $4 \mathrm{~mm}$ thick spacers of $256.5 \mathrm{~mm}$ in height and variable length. The space is filled with $3 \mathrm{~mm}$ scintillator.

\footnotetext{
${ }^{20}$ KURARAY Corp., 3-10, Nihonbashi, 2 chome, Chuo-ku, Tokyo, Japan.
} 
The periodic structure of the system is designed to be self supporting and uniformly instrumented with no dead zones. To facilitate the construction of modules, each module is subdivided into eight sub-modules that have a manageable size for assembly from the individual absorber plates. A total of 416 submodules have been produced to form 52 modules needed to build up the two halves of the HCAL structure. The mechanical structure is reinforced by welded cross members and is completely independent from the optical instrumentation.

The optics of the tile calorimeter consists of three components: the scintillating tile, the WLS fibre and a small square light mixer just in front of the photo-multiplier entrance window. The scintillating light propagates through the $3 \mathrm{~mm}$ thick tile to its edges where it is collected by $1.2 \mathrm{~mm}$ diameter WLS fibres.

In total more than 86000 tiles of two different dimensions have been produced by the cost effective casting technology using polystyrene as a base and two dopants: paraterphenyl (PTP, $1.75 \%)$ and POPOP $(0.05 \%)$. Each tile of $197 \times 256 \mathrm{~mm}^{2}$ or $197 \times 127 \mathrm{~mm}^{2}$ is wrapped in a 120-150 micron thick TYVEK envelope. ${ }^{21}$

The edges of a tile are wrapped in such a way that the fibre running along the edge can be easily inserted between the envelope and the tile edge during the module optics assembly. This reflective envelope avoids light crosstalk between adjacent edges of the small tiles, enhances the light collection in the wavelength shifting fibre and in addition protects the optical reflective surface of the tiles. Each fibre collects light from three scintillator tiles arranged along the shower development direction. There are a total of 50k fibres in the HCAL with an identical length of $1.6 \mathrm{~m}$. In order to increase the light collection efficiency both fibre ends were cut with a diamond mill, and opposite to the photomultiplier end coated with a layer of reflective aluminum deposited in vacuum. The reflectivity of all fibres was checked on a measuring stand specially designed for that purpose. All fibres were measured to have a mirror reflectivity in the range of $(85 \pm 10) \%$. The light propagates along the fibre by total reflection but tiles located further from the PMT yield less light due to attenuation in the WLS fibre. To compensate for this effect, the tile-fibre optical contact was progressively reduced for the different tile layers in depth. The last tile layer closest to the PMT has an optical contact reduced by $22 \%$ as compared to the first layer at the entry of the HCAL. This precaution minimizes the difference in response between tiles, providing light to the same PMT with a uniformity at a level of a few percent.

Each cell is read out by one photomultiplier tube that is attached to the rear mechanical structure of the module. The optical connection between the fibre bundle and the photomultiplier is ensured by a $35 \mathrm{~mm}$ long light mixer of square shape. To shield the photomultiplier tube from the stray magnetic field the tube, including the light mixer, is housed inside a $3 \mathrm{~mm}$ thick iron tube and MuMetal foil. The influence of the magnetic field on a PMTs performance was studied during the LHCb magnet measurement. A field of approximately $2 \mathrm{mT}$ along $z$, but negligible in $x$ and $y$, is expected in the region of the HCAL PMTs [23]. No variation of the monitoring LED signal has been observed within about $0.1 \%$ measurement error while switching on and off the magnet full power and when changing its polarity.

In order to monitor the HCAL stability an embedded self-calibrating scheme with ${ }^{137} \mathrm{Cs}$ gamma source has been implemented [147]. A capsule of $10 \mathrm{mCi}$ activity can be transported

\footnotetext{
${ }^{21}$ TYVEK of type 1057D used, product of E.I. du Pont de Nemours and Company.
} 


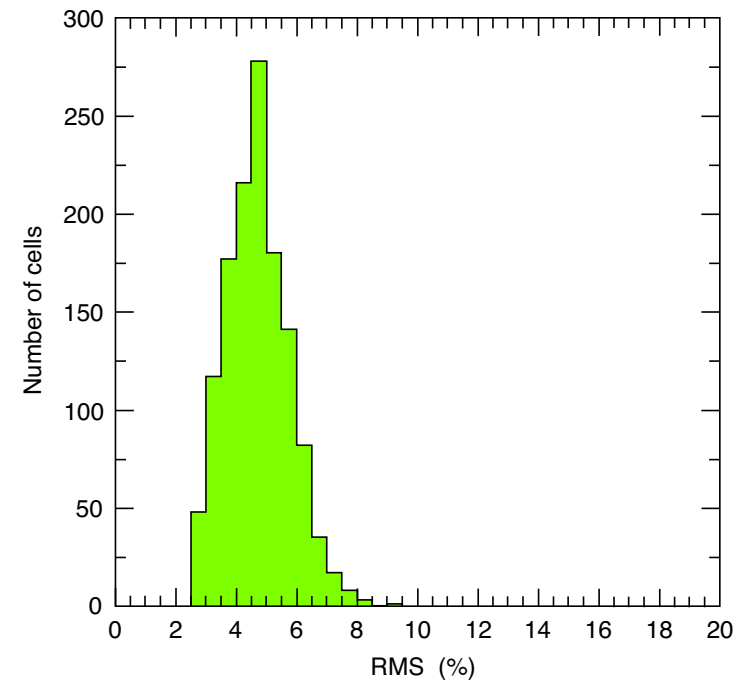

Figure 6.32: The distribution of RMS light yield for tiles read out by the same PMT.

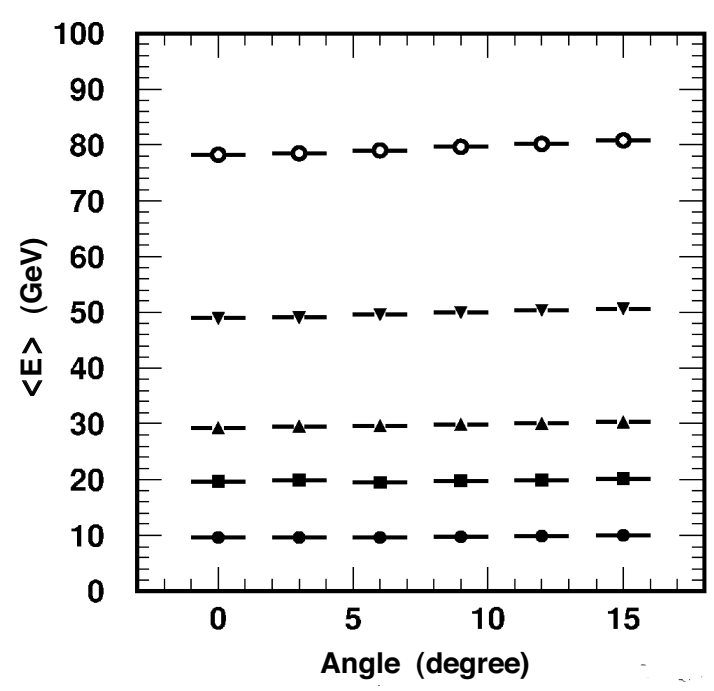

Figure 6.33: The angular dependence of the HCAL prototype response at different energies.

through a stainless steel pipe fed through all tiles. The source is encapsulated in a $2 \mathrm{~mm}$ diameter, $4 \mathrm{~mm}$ long stainless steel tube that is welded at both ends. The hydraulic driving system filled with distilled water includes a garage to store the source, and computer controlled pump and valves that allow reversal of the water flow direction. A separate readout circuit measures the integrated anode current every $5 \mathrm{~ms}$ resulting from the scan of a half detector when the source propagates sequentially through $26 \mathrm{HCAL}$ modules with an average velocity of about $20-40 \mathrm{~cm} / \mathrm{s}$. This procedure takes less than one hour. The method was widely used both during construction and in the final test of modules before installation in the cavern. Furthermore this system is used for the absolute calibration of the HCAL in-situ.

Beam tests performed with several HCAL modules allow the correspondence between the anode current induced by the radioactive source and the measured beam particle energy to be determined. Being absolutely calibrated the HCAL provides a unique possibility to cross-check the calibration of the upstream detectors (e.g. electromagnetic calorimeter) by comparison of the average energy deposition of the hadronic shower in the corresponding cells of two detectors. The energy sharing function varies slowly over the calorimeter surface and can be extracted from the MonteCarlo simulation.

The tile response during optics assembly was monitored using the radioactive source system. A maximum deviation of $20 \%$ from the average anode current for tiles grouped to the same PMT was accepted. The tiles and fibres not satisfying this requirement have been replaced. A distribution of the RMS of tile responses within the groups coupled to the same PMT is shown in figure 6.32. The mean value of this distribution is $4.7 \%$. 


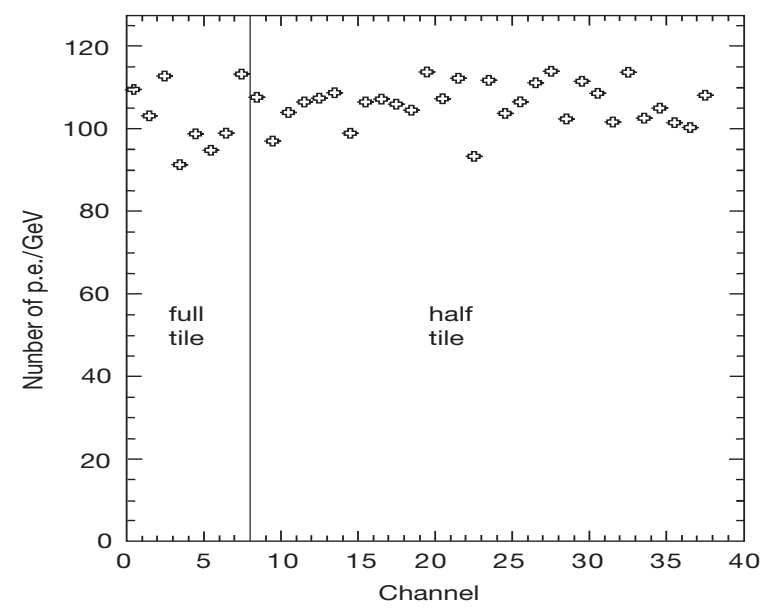

Figure 6.34: The light yield for the HCAL module measured in the test beam. The left part of the plot corresponds to 8 big cells of $26 \times 26 \mathrm{~cm}^{2}$. The other 32 cells comprise half-size cells of $13 \times 13 \mathrm{~cm}^{2}$.

\section{Performance of the HCAL modules}

The performance of the HCAL has been studied with prototypes [148] and continuously checked during module assembly using the CERN SPS test beam [149]. The dependence of the HCAL response on the angle of the incoming particle has been studied by rotating the detector between $0^{\circ}$ and $15^{\circ}$ around a vertical axis that traverses the HCAL close to the average shower maximum. Figure 6.33 shows the response of the $5.6 \lambda_{I}$ long prototype for pion energies between $10 \mathrm{GeV}$ and $80 \mathrm{GeV}$. From a lateral scan of the particle beam across the prototype front surface the uniformity in response is measured to be well within $\pm 3 \%$.

During the assembly process [150] tiles with similar properties were grouped into the same cell to minimize nonuniformities. The light yield of all cells from one typical module is shown in figure 6.34. In total five modules (out of 52) were tested in the beam with an average light yield of 105 p.e./GeV.

The moderate requirements for the HCAL energy resolution allow the ratio of the active to passive material in the detector to be as low as 0.18. Furthermore, owing to the limited space available, the length of the HCAL has been chosen at 5.6 $\lambda_{I}$. The upstream ECAL adds a further $1.2 \lambda_{I}$. Beam-test measurements were compared in detail with results using different software packages for the simulation of the hadronic shower development. The energy response to $50 \mathrm{GeV}$ pions is shown by the hatched histogram in the left-hand plot of figure 6.35. The tail towards low energies due to leakage of the shower is easily seen. However, this tail is not a concern for the hadron trigger performance since it only introduces some minor inefficiency for high $E_{T}$ signal events but does not affect the rejection of low $E_{T}$ minimum-bias events. The black dots show the result of a MonteCarlo simulation that is in good agreement with the data, when using the GEANT (MICAP+FLUKA) interface. The energy resolution has been determined by fitting the energy spectrum with a gaussian distribution bounded by $\pm 2.5 \sigma$. The resolution extracted from a fit to the data at several energies is $\sigma_{E} / E=(69 \pm 5) \% / \sqrt{E} \oplus(9 \pm 2) \%(\mathrm{E}$ in $\mathrm{GeV})$ as shown in the right-hand plot of figure 6.35 . 

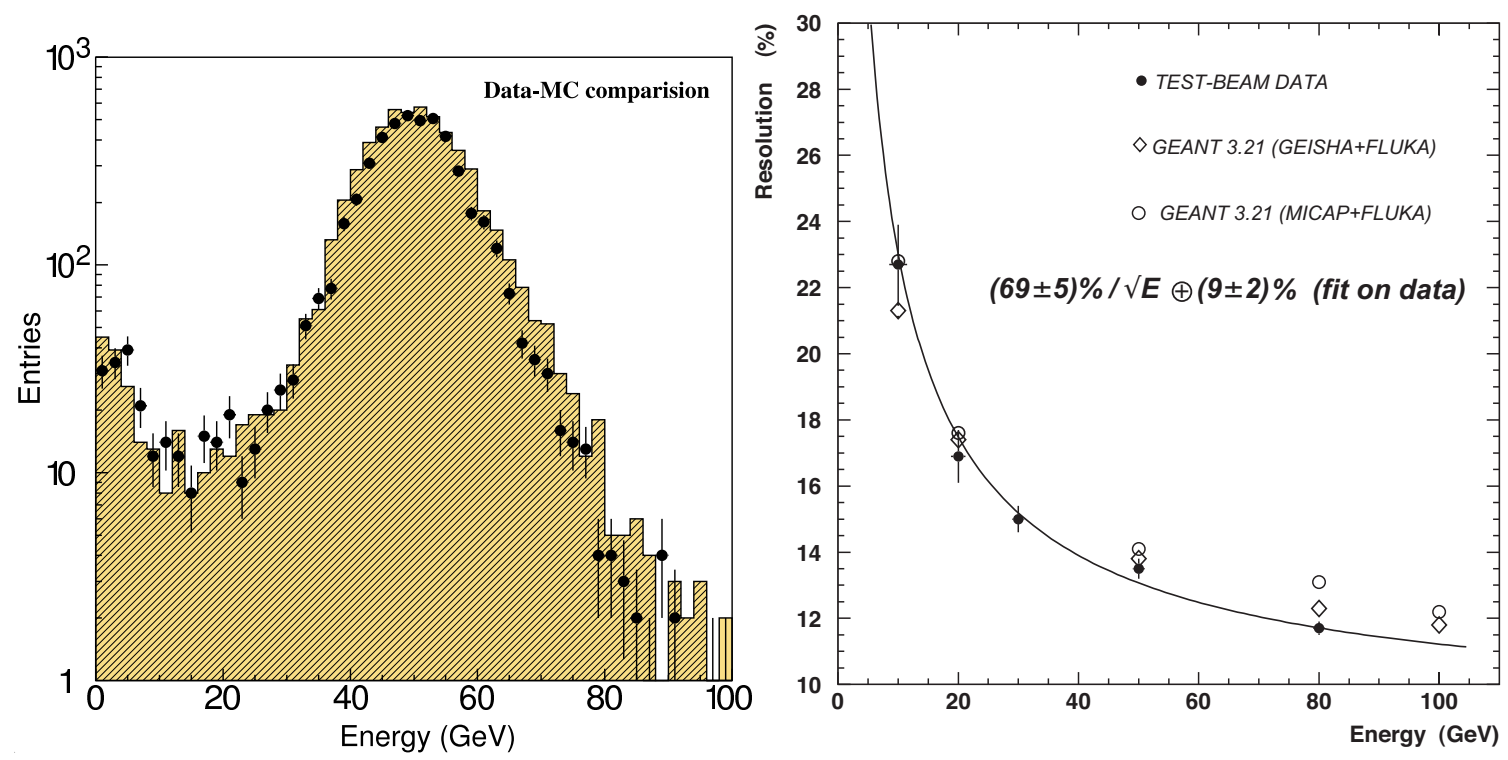

Figure 6.35: Left: energy response for $50 \mathrm{GeV}$ pions from test-beam data (hatched) and from simulation (dots). Right: HCAL energy resolution, both for data and for simulation with three different hadronic simulation codes. The curve is a fit to the data.

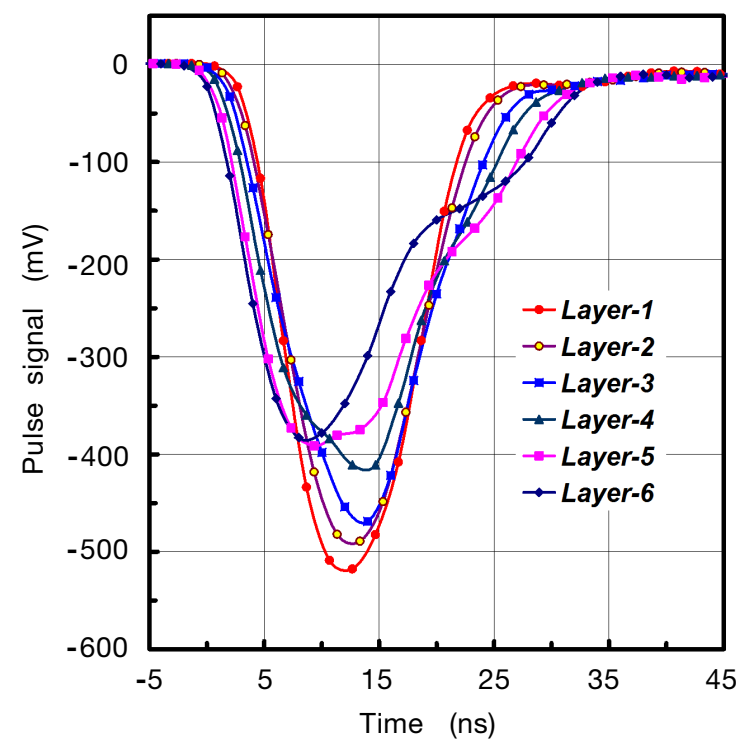

Figure 6.36: The average signal pulse shape of $30 \mathrm{GeV}$ electrons in tile layers at different depths.

Another important feature of the HCAL is its signal timing properties. A precise signal shape measurement has been done with an electron beam of $30 \mathrm{GeV}$ hitting layers of tiles at different depths. For this purpose the HCAL modules have been rotated by $90^{\circ}$ and moved transversely with respect to beam line. Average pulse shapes of the detected signals are shown in figure 6.36. The 
variation of the shape is due to light reflection from the mirror at the end of the fibre opposite to the PMT. For layer 1, for example, the direct and reflected light reach the PMTs simultaneously, but for layer 6, closest to the PMT, the direct light reaches the photocathode earlier than the reflected one by the propagation delay in the $1.2 \mathrm{~m}$ long WLS fibre.

\section{Radiation resistance of the HCAL modules}

A comprehensive study of the degradation under irradiation has been performed for all optical components of the HCAL [151]. Scintillating tiles and WLS fibres have been irradiated at the IHEP $70 \mathrm{GeV}$ proton synchrotron near the internal target area to get realistic environmental conditions as expected during the LHC operation. The transparency of tiles degrades by $25 \%$ after $250 \mathrm{kRad}$ irradiation, but then steadily decreases by a further $20 \%$ with irradiation up to $1.4 \mathrm{MRad}$. The relative light yield after irradiation shows almost the same behaviour. Another important effect is the radiation degradation of the WLS fibres which introduces a longitudinal nonuniformity of the calorimeter response [152]. For example, the attenuation length in the WLS fibre degrades by $30 \%$ after irradiation with a dose of about $0.5 \mathrm{MRad}$, which corresponds to 10 years of operation for the most central cells of the HCAL at nominal luminosity of $2 \times 10^{32} \mathrm{~cm}^{-2} \mathrm{~s}^{-1}$. As a result of the same amount of irradiation, the constant term of the HCAL resolution degrades by $20 \%$. To monitor the fibres radiation damage we intend to use the embedded calibration system with the radioactive source, that allows measurement of the response from each tile along the detector depth with a few percent accuracy.

\subsubsection{Electronics of ECAL/HCAL}

Because of the similarity of input signals and functionalities, it has been decided to use a common electronic system for the ECAL and HCAL. This system is based on a deadtimeless and low pedestal integrator system using delay lines, followed by ADCs and pipeline buffers. Each card is connected to 32 channels of the ECAL or the HCAL. After digitization in the front-end card the data is processed further and sent to a trigger validation card. The data is also pipelined and stored on the front-end card, and then sent through the back plane to a calorimeter readout card (CROC).

\section{Pulse shaping and front-end chip}

In order to take advantage of the inherent speed of the scintillator and WLS based calorimeter system the electronic system is capable of measuring every bunch crossing independently of signals from associated neighboring bunch crossings (pileup). The PMT pulses therefore are shaped to eliminate the small tail of pulses extending beyond $25 \mathrm{~ns}$. A schematic of the PMT signal shaping and of the front-end chip is shown in figure 6.37 for HCAL. The PMT pulse is shortened by a clipping line which is a cable for the HCAL and a delay chip for the ECAL due to lack of space. The length of the line is typically one metre, giving a FWHM of about $10 \mathrm{~ns}$. The resistor load at the end of the line is adjusted to obtain a return to a 0 Volt level after clipping. Due to fluctuations in the number of photoelectrons as a function of time, a fluctuation is also present in the shaped pulse. However the effect is small, especially for the ECAL where about 3000 photoelectrons produced per $\mathrm{GeV}$. The pulses are then sent along $10 \mathrm{~m}$ of coaxial cables to the front-end cards which reside in 


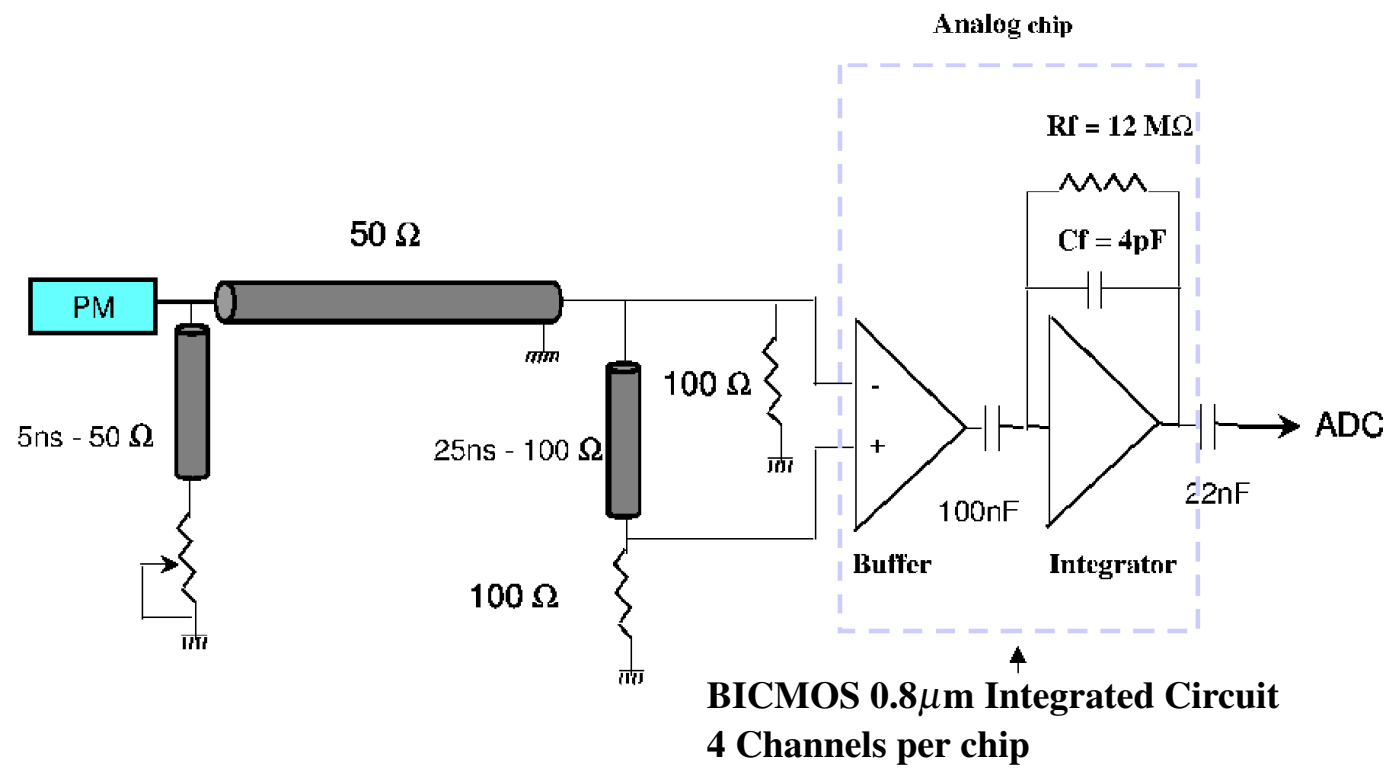

Figure 6.37: Schematic of the PM signal shaping and of the front-end chip.

crates above the calorimeters. The pulse-shape distortion produced by the cable is compensated to first order by a pole-zero circuit placed after the integrator. The first element of the pole-zero card is a front-end chip which contains a buffer amplifier and an integrator. To discharge the integrator the same pulse is subtracted in a buffer amplifier after a $25 \mathrm{~ns}$ delay obtained by a lumped-element delay line. Simulated input and output pulses using a $10 \mathrm{~ns}$ rectangular pulse are shown in figure 6.38, as well as an average $80 \mathrm{GeV}$ PM HCAL pulse (averaged over 1000 measurements on a digital scope) and the resulting integrated pulse.

The schematic of the analog chip is given in figure 6.39. The circuit has been realized in AMS $0.8 \mu \mathrm{m}$ BICMOS technology. A typical noise of $150 \mu \mathrm{V}$ has been obtained after a $25 \mathrm{~ns}$ integration, which should correspond to a total noise of less than one ADC count. As measured with prototypes, the tail of the pulse integral in the next sampling is about $2 \%$ with an additional fluctuation of $1 \%$. The impact of the amplifier noise on the physics performance is found to be negligible compared to the energy resolution of the ECAL.

\section{The ECAL/HCAL front-end board}

Figure 6.40 shows the block diagram of the 32 channel front-end board. It is a $9 \mathrm{U}$ board using VME mechanics with a specialized serial bus used for Experiment Control System purposes (see section 8.4). The pulses from the front-end chip, described above, are sent to 12-bit ADCs. The pulses after integration are flat (within $1 \%$ ) over $\pm 2 \mathrm{~ns}$ around the maximum. To strobe at the maximum of the pulse, the different delays in the photomultipliers have to be compensated. This is achieved by adjusting the clock of each ADC by a delay chip to an accuracy of $1 \mathrm{~ns}$. The digitized output of the ADCs are then resynchronized to a common clock (per card) in a register at the input of the next chip. The pulses at the integrator output can be subject to baseline shift and to the 

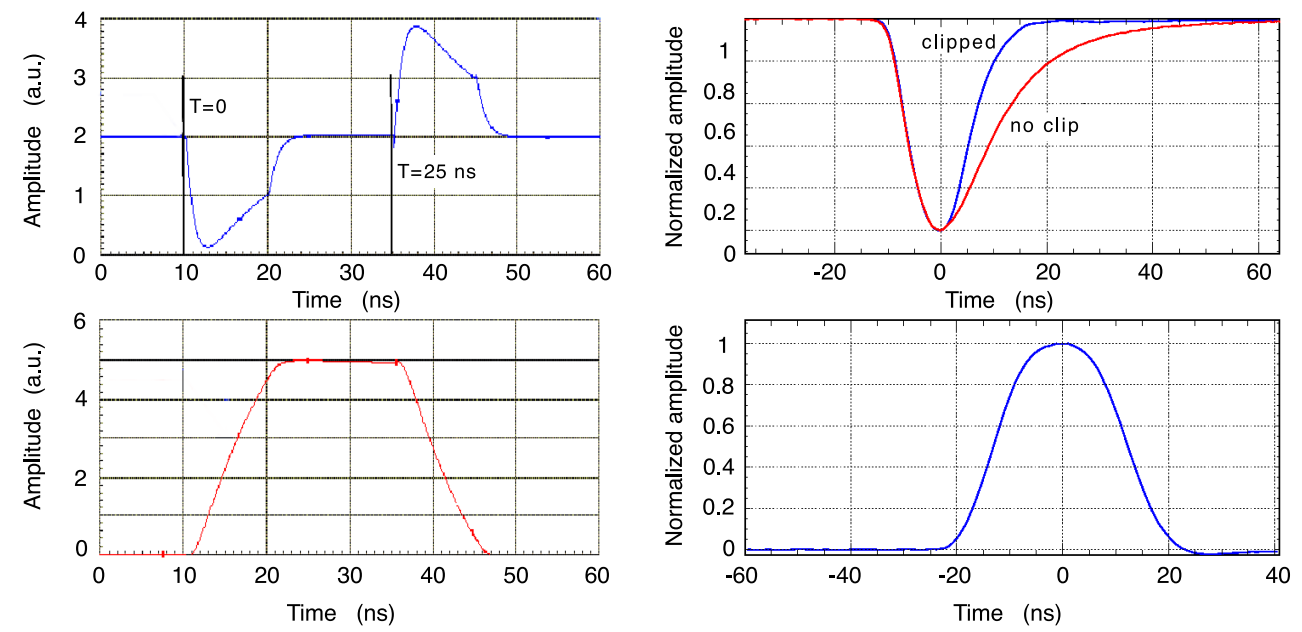

Figure 6.38: Left: simulated input pulse of a $10 \mathrm{~ns}$ rectangular pulse and the corresponding output pulse of the integrator: buffer output current before integration (top) and output integrated signal (bottom). Right: $80 \mathrm{GeV}$ HCAL signals. The upper figure shows the PM signal with and without clipping, the lower one shows the same clipped signal at the output of the integrator.

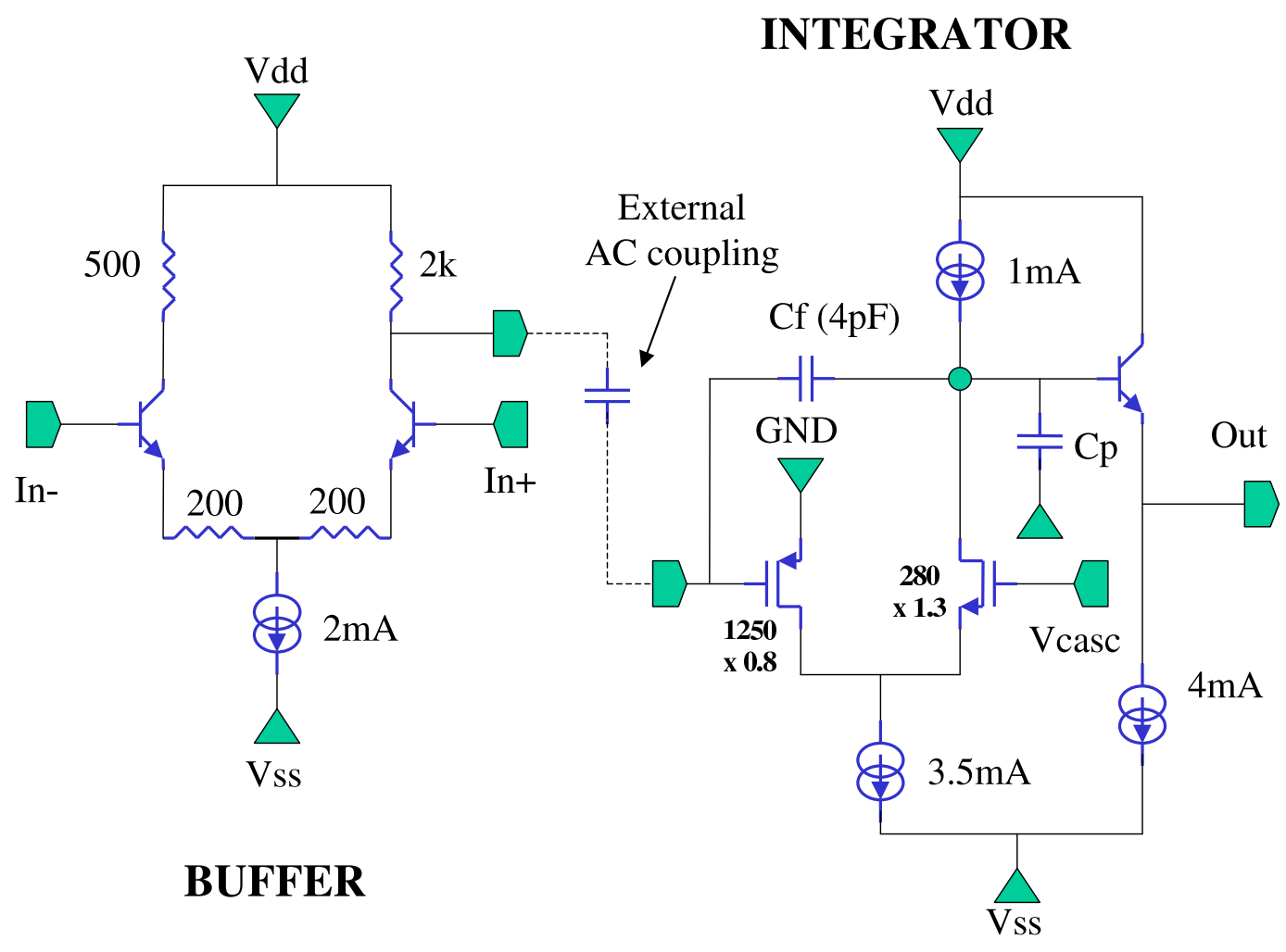

Figure 6.39: Schematic of the analog chip.

influence of low frequency pickup noise which would cause slow variation of the ADC pedestal. These variations are cancelled through a digital differentiation by subtracting the digitization in a 


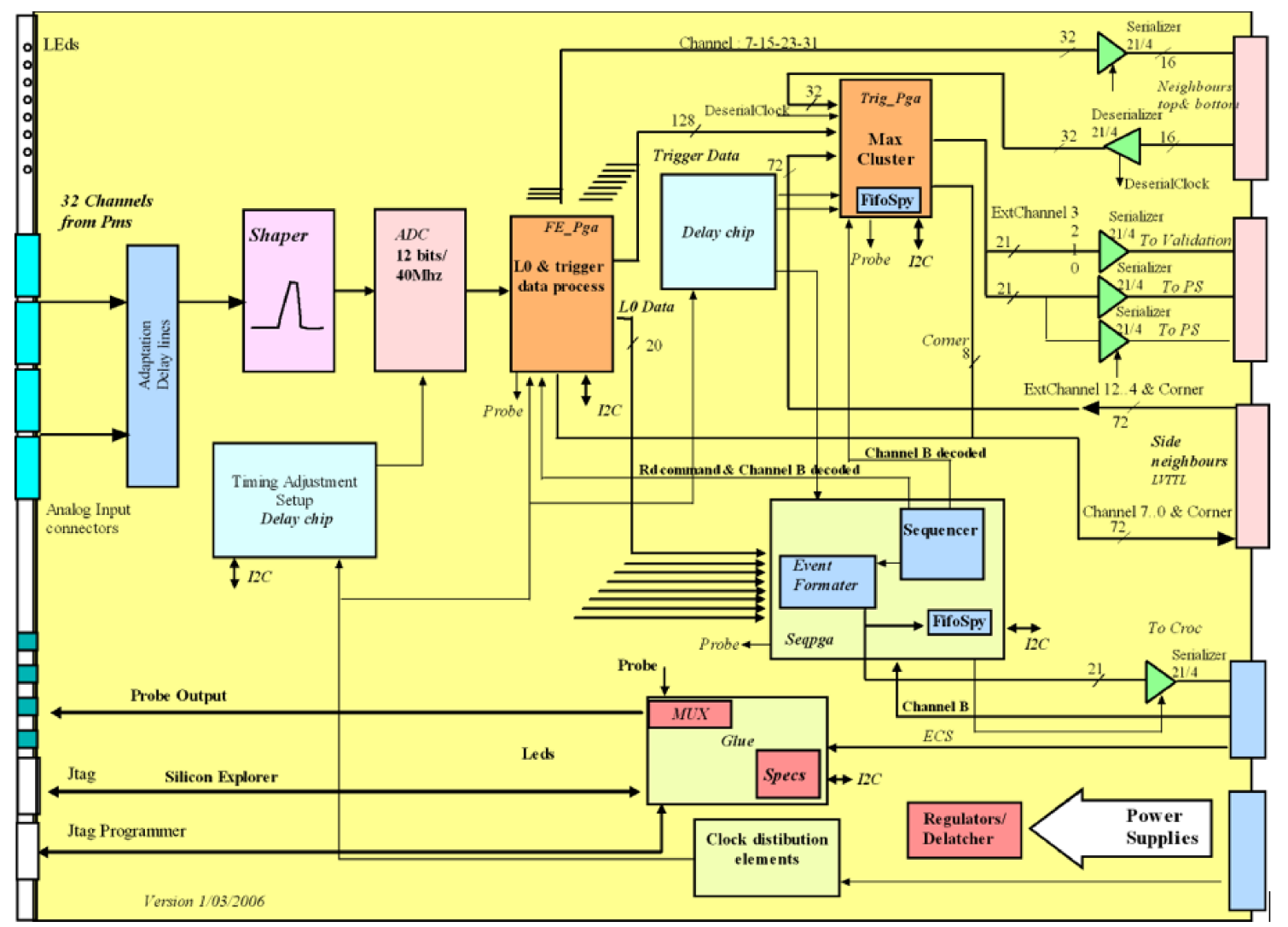

Figure 6.40: Block diagram of the front-end board, for ECAL and HCAL.

preceding sample ( $25 \mathrm{~ns}$ before). This subtraction also defines the effective integration time of the amplifier and therefore limits the integrated thermal noise from the buffer amplifier. To decrease the possibility of subtracting a signal present in the same channel, the quantity subtracted is actually the smallest of the two preceding measurements. Even at the highest occupancy, about 5\%, the probability of subtracting a significant signal is therefore reduced to less than $0.25 \%$. Simulations have shown that this procedure introduces negligible deterioration of energy and position measurements [129]. However the procedure introduces a pedestal shift of about $40 \%$ of the $40 \mathrm{MHz}$ noise (i.e less then one ADC count) and an increase of the $40 \mathrm{MHz}$ noise by $16 \%$. The subtraction of the smallest of the two preceding samples is performed in 8 dedicated anti-fuse based FPGAs (cf. section 7.1.3) ${ }^{22}$ called FE-PGAs, each of these being used to process four ADC channels [153]. Actually each FE-PGA has, for each ADC channel, four functional blocks. The first one processes the input ADC data, as described above. The second block produces the trigger data, converting the 12 bits of the ADC to 8 bits by a simplified multiplier with an adjustable 8 bits calibration constant. The third block is in charge of storing the data (12 ADC bits and 8 trigger bits per ADC, 80 bits per FE-PGA), during the L0 latency, and upon L0-Yes store again the data in a derandomizer and send them as four successive groups of 20 bits to the SEQ-PGA. The last block of the FE-PGA permits injecting test values at the input, in place of the ADC values, in order to check the proper behaviour of the card.

\footnotetext{
${ }^{22} \mathrm{AX} 250$ anti-fuse PGA from ACTEL.
} 
To adjust the timing of the clock signals used to strobe the ADCs and to synchronize the PGAs, delay-chip modules are used. These delay chips are custom made ASICs which shift the $40 \mathrm{MHz}$ clock sent by the crate controller, the CROC. The delay-chips' clock shifts have a $25 \mathrm{~ns}$ range and steps of $1 \mathrm{~ns}$.

Three other PGAs are used on the board for specific functions: The Trig-PGA is used to process the 8 bits trigger data from each of the 32 channels of a board together with the 8 sideneighbours channels from adjacent boards 4 top neighbours and one corner neighbour from other crates. The Trig-PGA computes the total transverse energy of the board and the information on the $2 \mathrm{X} 2$ cluster of cells with the highest transverse energy. This information is then passed to further boards involved in the trigger decision (the PS/SPD FE Board and the Trigger Validation Board). The Trig-PGA is implemented in an anti-fuse AX500 from ACTEL. The SEQ-PGA, mentioned above, serializes the 32 channels of the front-end board and sends them to the readout controller, the CROC. It also dispatches the L0 trigger signals and control signals linked to the trigger, the socalled channel B, to the FE-PGAs. The Glue-PGA is an interface between the Experiment Control System (based on the SPECS system) and the other PGAs of the board. It allows to load and read back constant parameters which adjust the delay chip values, calibration constants in FE-PGAs and so on. The SEQ and Glue PGAs are implemented in reprogrammable flash-based PGAs from ACTEL the APA300 and APA150.

\section{The calorimeter readout card (CROC)}

The CROC (for a conceptual design see [129]) is the board, which gathers the front-end L0 data of a calorimeter crate and sends them through optical fibres to the LHCb DAQ system (the TELL1 boards). Each of the 26 crates housing up to 16 ECAL, HCAL or PS/SPD front-end boards, are equipped with one single CROC, plugged into the central slot. The CROC also provides the clock, trigger signal, broadcast (channel B) and slow control command for all boards of a crate. In the DAQ path the data from front-end boards, which has been serialized at a $280 \mathrm{Mbits} / \mathrm{s}$ rate and sent through the backplane, are first deserialized and then captured and resynchronized in 4 FE-PGAs. These FE-PGAs (APA300) detect the presence of data (header detection) and add to the 21 bits of data coming from front-end boards 11 bits providing the data type and the board and crate identification numbers. The data are then sent to 2 GOL mezzanines each equipped with 8 fibres. In the absence of data the FE-PGAs send a special signal used to periodically synchronize the fibres drivers and receivers in the TELL1 boards. A spy functionality is also implemented in the FEPGAs where data for up to $15 \mathrm{~L} 0$ events can be copied into RAMs. The data are then collected by a dedicated Spy-PGA based on an APA450. These dcalorimeter system is inherently fast, it has been decided to ata can be read at a slow rate using a slave mezzanine of the SPECS ECS system, which is mounted on the CROC board. The SPECS mezzanine also loads and reads control data onto the board, and also sends through the backplane, ECS data to the Glue-PGAs of each front-end board which are programmed to act as SPECS slaves. Finally a TTCrx mezzanine mounted on the CROC board receives by a fiber the $40 \mathrm{MHz}$ clock, the L0 trigger signal and the channel B signals which have been generated in the readout supervisor boards in the electronic barracks. These signals are buffered and sent through the backplane to each other board in the crate. For debugging purposes the CROC can work as a stand alone system locally generating the clock L0 and channel B signals. 


\section{Calorimeter specific TELL1 features}

The TELL1 boards of LHCb are described in section 8.2. The specifications of the calorimeters are described in [154].

A preprocessor FPGA (PP-FPGA) collects and treats the incoming data from front-end boards sent by the CROC to the TELL1 board through an optical receiver mezzanine card (O-Rx card) which ensures the optical to electrical conversion and data synchronization. The PP-FPGA treats the event according to the detector identification (ECAL/HCAL or PS/SPD). Inside each PP-FPGA, a zero suppression is performed on the events during physics runs for PS/SPD data and a data compression without loss of information for ECAL and HCAL data [155]. The output data of each PP-FPGA are collected by the SyncLink-FPGA which then packs them in a Multiple Event Packet (MEP). For each event, the SyncLink-FPGA gives the trigger type of the run (physics or calibration) to the PP-FPGA. The MEPs are sent to the DAQ through a Gigabit-Ethernet (GBE) card. The output bandwidth of the GBE limits the use of one among the two O-Rx cards for ECAL and HCAL and thus two PP-FPGAs among the four. The two O-Rx cards are used for PS/SPD. In case of no data and/or synchronization error from the optical input links in the O-Rx card, an error bank is optionally generated (it is suppressed by default due to bandwidth limit). In order to keep track of these errors, a minimal information is kept in each event header.

The LED monitoring system is described in section 6.2.8. During calibration runs the LED data are not affected by the zero suppression, and only non-zero values are kept for PS/SPD. During physics runs, the data coming from the PIN boards (FE boards devoted to the PIN diode signals of ECAL/HCAL) are ignored.

\subsubsection{Electronics of PS and SPD detectors}

The signal of the PS and SPD has about the same duration as the ECAL and HCAL pulses; on average $85 \%$ of the charge is obtained in $25 \mathrm{~ns}$. However, since the average number of photoelectrons is only about 25 per MIP, there are very large fluctuations in the signal pulse shape [156]. It was therefore considered unreasonable to try to implement the delay-line pulse shaping used for the ECAL/HCAL. On the other hand, since the useful dynamic range of the PS is typically only from 1 MIP to 100 MIP, an ADC with only 10-bits can be used and pedestal stability is not as important as for the ECAL/HCAL electronics, although it is important to integrate the signal over a time as long as possible within the $25 \mathrm{~ns}$ limitations. The phototube chosen to detect the light from the PS and SPD fibres is a 64 channel multi-anode PM. The HV is common to all 64 channels and there is a nonadjustable gain dispersion among these channels of a factor of 3 , which however is constant in time. For this reason it was decided to introduce a Very Front End (VFE) stage of integration on the back of the phototubes in order to compensate the gain variation using a digital correction of a factor up to 2 in the front-end electronics.

\section{The PS very front-end design}

The solution adopted for the PS is to alternate every $25 \mathrm{~ns}$ between two integrators and to reset one integrator when the other one is active. The signal is sampled by track-and-hold circuits and the output of the active integrator is chosen by a multiplexer, followed by a twisted-pair cable driver. 


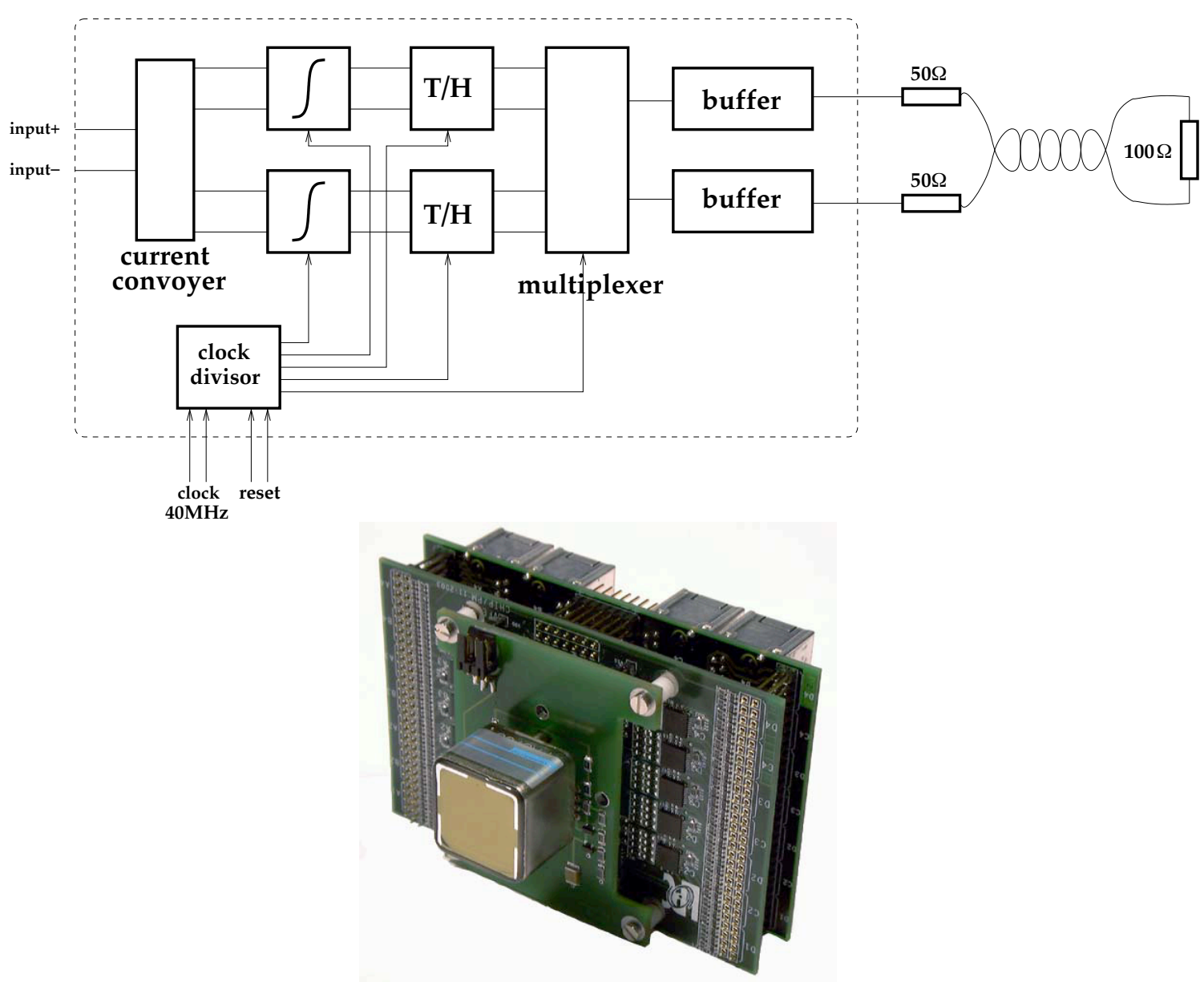

Figure 6.41: Schematic of the PS Very Front-End integrator (top) and a photograph of the integrator card mounted behind the MAPMT (bottom).

All circuit elements are functioning in differential mode to improve stability and pickup-noise rejection. The circuit design is shown in figure 6.41 and its detailed description is given in [157]. The phasing of the clock with respect to the signal determines the start of the integration. Since the length of the 64 fibres connected to a given PM are identical and the delay inside the PM is identical within a fraction of $1 \mathrm{~ns}$, it is sufficient to have one clock adjustment (in steps of $1 \mathrm{~ns}$ ) per phototube. The amplifier integrator circuit is realized in monolithic AMS $0.8 \mu \mathrm{m}$ BICMOS technology with 4 channels implemented per chip. The dynamic range is 1 Volt with a noise of $1 \mathrm{mV}$. Sixteen chips are grouped in a card on each phototube. The outputs of each chip (4 channels) are sent through $27 \mathrm{~m}$ long ethernet cables ${ }^{23}$ with RJ45 connectors to the ADC located on the FE board. Two clock and two reset signals delivered by the corresponding FE board are received by the VFE board through a cable of the same type as those used for the signal.

\footnotetext{
${ }^{23}$ from Kerpen company, Geramny.
} 


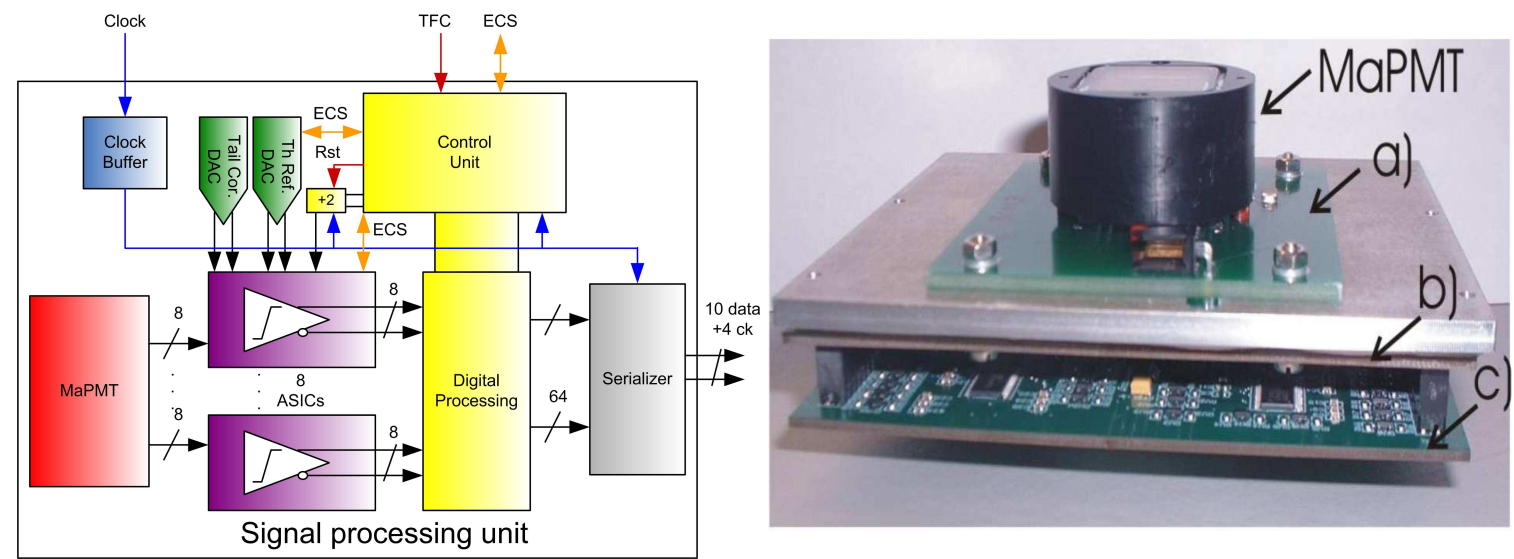

Figure 6.42: Left: block diagram of the SPD VFE card. Right: picture of the VFE assembly where the components are a) PMT base board, b) ASICs board and c) FPGA board.

\section{The SPD very front-end and control board design}

The SPD function is to discriminate between charged particles and neutrals in a detector which matches the preshower and ECAL cell size. The SPD signals are obtained, as in the case of the PS, from 64 channel multianode photomultipliers. The main difference of specification is that there is no compelling reason to perform pulseheight analysis on SPD signals. It was therefore decided to use a simpler solution with a simple discriminator output and a threshold set at 0.5 MIP thus obtaining 98\% efficiency on charged particles $[131,137]$. The principle of the design is shown in figure 6.42 and described in [127]. Although the functional architecture of a signal processing channel is similar to that of the PS, the circuits have been designed for the SPD specifically [158, 159]. The main architectural difference is the inclusion of an on chip pile-up compensation system and a comparator. The pile-up compensation system takes a tunable fraction of the integrator output at the current period and stores it on a track and hold circuit. This cancels on average the charge delivered by a pulse in the time interval between 25 and $50 \mathrm{~ns}$ after its start. The reference threshold value is set by a 7-bit DAC. The integration of each channel uses an independent DAC to compensate the offsets and the gain nonuniformity of the PMT. The circuit is realized in the AMS BICMOS $0.8 \mu \mathrm{m}$ technology with 8 channels per chip. There are two external (not on-chip) DAC, with $\mathrm{I} 2 \mathrm{C}$ interface to set some analogue references for the signal processing part. The control unit, the digital processing and clock divider used to obtain the $20 \mathrm{MHz}$ clock that controls the ASICs are implemented in a reprogrammable FPGA, the ProASIC Plus ACTEL APA300. Triple voting registers (TVRs) are used to minimize signal event upset (SEU) errors. The digital processing consists of mapping PMT channels to given serializer channels to match the PS and SPD detector cell and injecting arbitrary patterns to test the detector data flow. The 64 channels and the $20 \mathrm{MHz}$ clock are sent via standard shielded twisted pair (SSTP) LAN cables to the PS/SPD FE board, by means of 4 serializer chips (DS90CR215) with 3 data pairs and 1 clock pair each, requiring a serializing factor of 7. Control boards provide the VFE cards with links to the ECS and the timing and fast control (TFC) system. The boards are located in the FE crates. Two boards are needed for each of the 8 crates corresponding to the PS/SPD subsystem. 


\section{The PS/SPD front-end board}

The PS/SPD front-end boards implement functions similar to the HCAL/ECAL boards i.e an ADC for the preshower signal, pedestal correction and gain calibration, preparation of the trigger information, pipeline storage and selection of data for readout upon reception of trigger signals. The PS/SPD board handles 64 PS data channels for raw data readout and trigger purposes, and 64 SPD single bit trigger channels. The PS raw data dynamic range corresponds to 10 bits, coding energy from 0.1 MIP(1 ADC count) to 100 MIPs. A general overview of the board is given in figure 6.43 for the PS. An identical board is used for the SPD. Its general architecture is similar to the ECAL/HCAL boards with five major components:

- An analog block receiving the 64 analog PS channels from the VFE part and digitizing them. Each channel is composed of a fully differential operational amplifier followed by a 10-bit $40 \mathrm{MHz}$ differential ADC. A synchronization signal (clock and reset) is sent to the VFE.

- A processing block made of 8 identical FE PGAs. Each of them is in charge of processing 8 PS channels. After applying corrections for pedestal subtraction, gain adjustment and pile-up, the 10-bit data are coded into an 8-bit floating format. A trigger bit is produced for each channel by applying a threshold on the corrected data. Eight SPD channels are also received. Two PS and SPD channels are packed together, stored and retrieved after L0. Two blocks of memory per two channels are used in each FE PGA for the L0 pipeline and derandomizer. The processing block is very resource consuming. Since the VFE comprises two interleaved integrators working in alternance, the gain and offset corrections have to be applied differently for two consecutive events leading to two effective subchannels per physical channel. Also the data inputs, 8 SPD channels of 1-bit and 8 PS channels of 10 bits are important. Consequently, the FE PGA chosen was the AX1000 of the ACTEL anti-fuse technology.

- A trigger block made of one Trig-PGA. It handles the processing for the production of the L0 information. The block receives the address of each cell and its local maximum of transverse energy from the ECAL Front End Board (FEB). As the ECAL electronics is organized into 32 channel boards, each 64 channel PS/SPD FEB is seen by the system as two 32 channel half boards, each receiving its own request address. The Trig-PGA is an APA450 of the ACTEL ProASIC plus Flash based FPGA family.

- A SEQ PGA builds the data block after a L0-Yes signal, and sends it to the CROC. It also issues control and synchronization signals for the other 8 FE PGAs and the Trig-PGA.

- A SPECS slave called GLUE PGA handling all the I2C communication of the board. The last two blocks, SEQ PGA and GLUE PGA, are identical to the ones of the ECAL/HCAL FE electronics and are described in reference [128].

All registers holding permanent information are radiation protected in static mode by triple voting and, during data transfers, by Hamming code. 


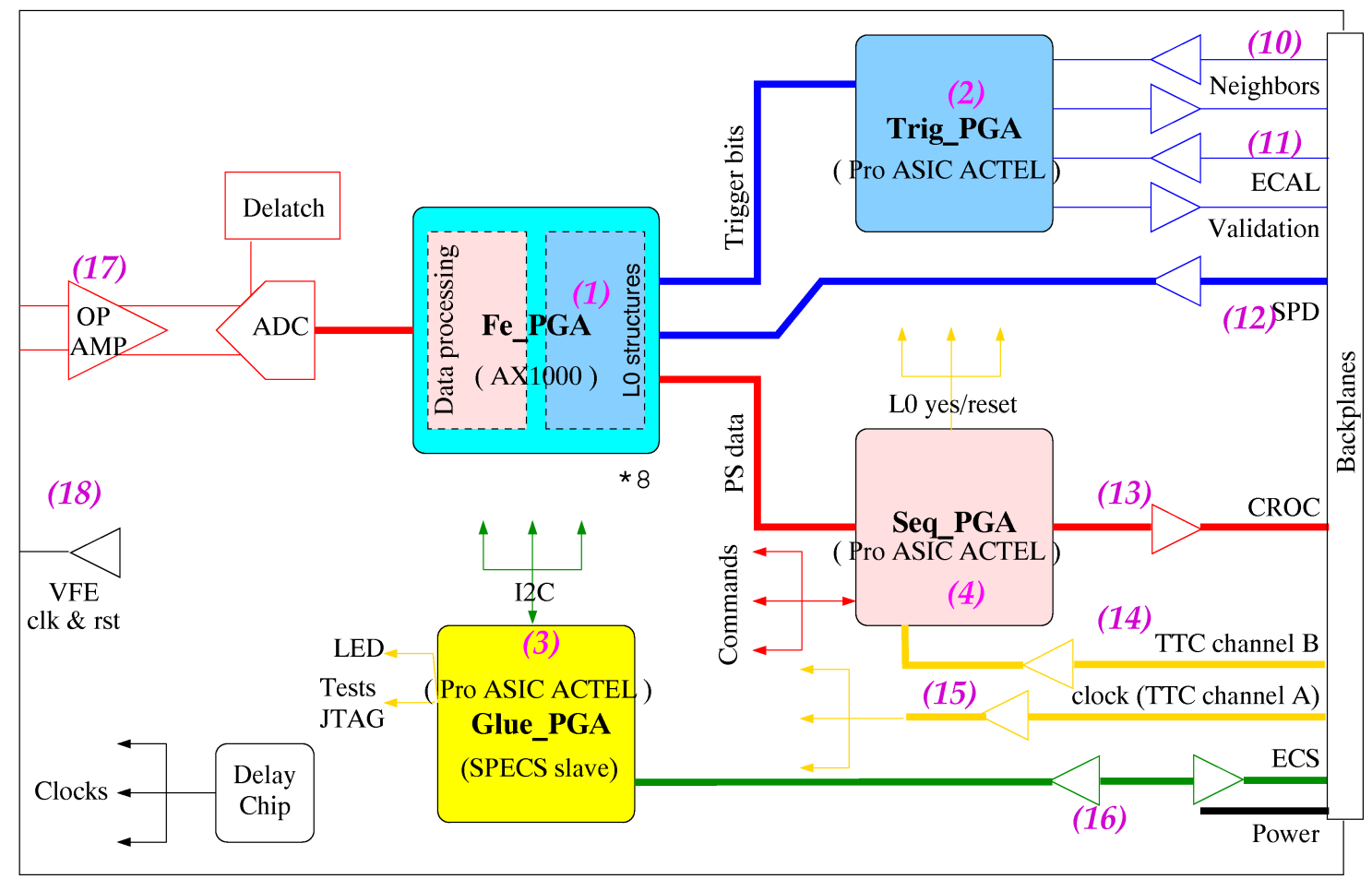

Figure 6.43: Schematic of the front-end board for the PS.

\subsubsection{Monitoring and high voltage system}

The calorimeter High Voltage (HV) and LED light intensity control systems share a common control board, the HV-LED-DAC board, that interfaces them to the Experiment Control System (ECS).

\section{The High Voltage system}

All calorimeter subdetectors are equipped with Hamamatsu photomultipliers. Eight thousand R7899-20 tubes are used for ECAL and HCAL and two hundred 64 channels multi-anode R760000-M64 for the SPD/PS detectors. All PMTs passed quality checks on dedicated test benches before installation on the detectors. The quality requirements included a better than $5 \%$ linearity and better than $3 \%$ gain stability as a function of rate, a low dark current and, for the SPD/PS PMTs, pixel-to-pixel uniformity of better than 1 to 3 (minimum to maximum) and crosstalk of less than $2 \%$.

The HV system for the PMTs (figure 6.44) is based on a Cockroft-Walton (CW) voltage converter located on the base of each PMT and controlled by HV-LED-DAC control boards located in a few boxes distributed over the detector periphery and connected to the (ECS) by a SPECS [160] serial bus. For the SPD and PS the CWs and control boards are located in a VME crate on top of the ECAL and the HV is distributed to the PMTs with cables. The maximum HV is $1500 \mathrm{~V}$ for ECAL and HCAL and $800 \mathrm{~V}$ for SPD and PS. The CW base [161] is a reoptimized and radiation hard version of the one used in the HERA-B experiment [162]. It is implemented on a $25 \mathrm{~mm}$ x $60 \mathrm{~mm} \mathrm{CW}$ board and consists of 22 multiplication stages, a control operational amplifier, an 


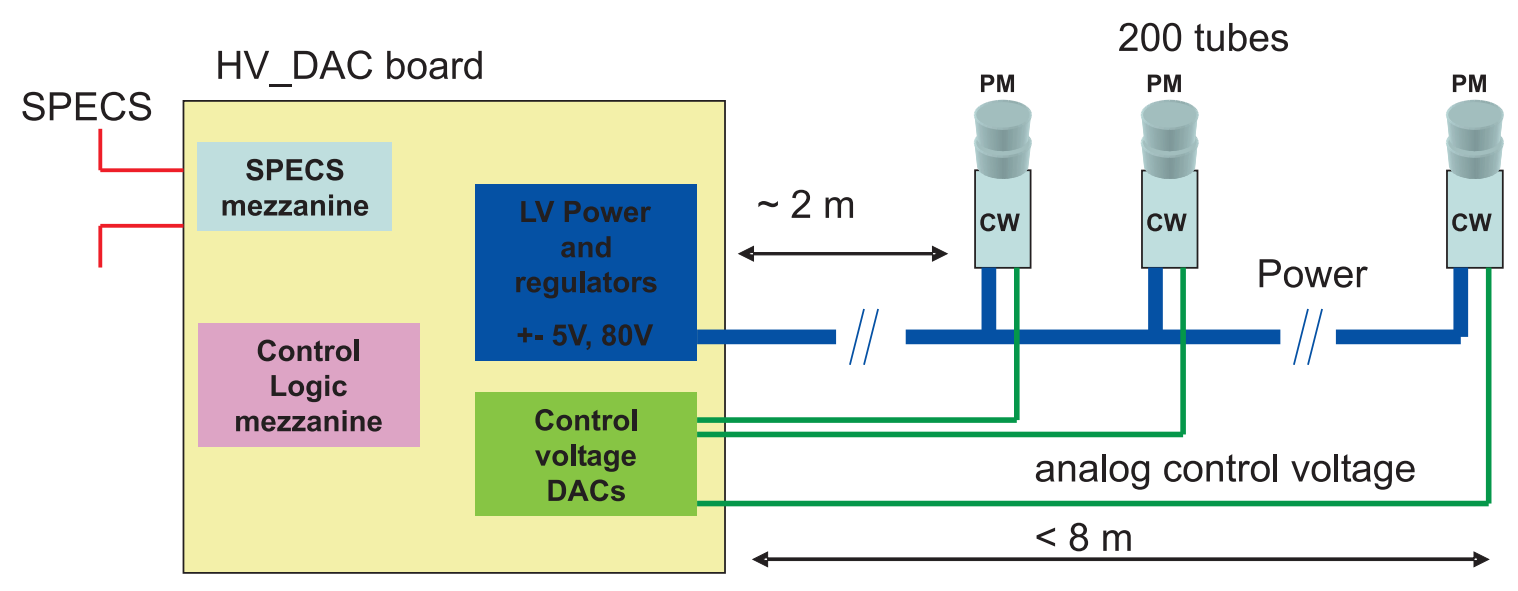

Figure 6.44: An overview of the High Voltage system of the calorimeter.

oscillator of $50 \mathrm{kHz}$ and fast transistor switches. It features individual and precise gain adjustment of each PMT, effective operation at high background anode current, low power dissipation and only low voltage $( \pm 6 \mathrm{~V})$ power supplies. It requires, in addition, $100 \mathrm{~V}$ which is converted locally to a high voltage in the $100-2000 \mathrm{~V}$ range selectable through a 0 to $5 \mathrm{~V}$ control voltage supplied by the control board. This conversion, being made locally for ECAL and HCAL, minimizes the number of high voltage cables and connectors. The characteristics of the $\mathrm{CW}$ are a gain drop of less than $2 \%$ for anode currents as high as $12 \mu \mathrm{A}$, a voltage ripple of less than $200 \mathrm{mV}$, a spread of output voltages of about $1 \%$ and a power consumption of less than $130 \mathrm{~mW}$. Each HV-LED-DAC board can control $200 \mathrm{CWs}$ and consists of a mother board and four mezzanine cards. A SPECS slave mezzanine is used for interconnection to the ECS system. The 200 PMTs are controlled by five 40channel HV control signal generating cards using 12-bit DAC integrated circuits. A control logic board equipped with a radiation tolerant FPGA ACTEL APA075 provides an interface between the ECS and the 200 DAC signal generating integrated circuits. This board also allows automated monitoring and ramp-up of the high voltage. The fourth mezzanine card generates a 16 channel LED intensity control signal.

\section{The LED monitoring system}

The energy calibration of each cell of the ECAL calorimeter is obtained during data-taking by a variety of methods, such as monitoring their response to electrons, $\pi^{0}$,s and minimum ionizing particles. ECAL and HCAL stability is monitored using LEDs, in order to obtain the best energy resolution essential for both triggering and reconstruction issues. The concept of the sytem is outlined in figure 6.45. Each cell of the four subdetectors is illuminated by an LED. They are triggered by LED drivers (labeled LED in figure 6.45) and their intensity controlled by the HV-LED-DAC boards. Multichannel LED Trigger Signal Boards (LEDTSB) perform the overall control and adjust the timing. For SPD and PS an individual LED is located on each tile and illuminates the PMT through the scintillator. Groups of 16 LEDs are controlled by 770 LED drivers located on the supermodules which are fired by 100 trigger channels.

On ECAL, LEDs are located in light-tight boxes situated above and below the calorimeter. 


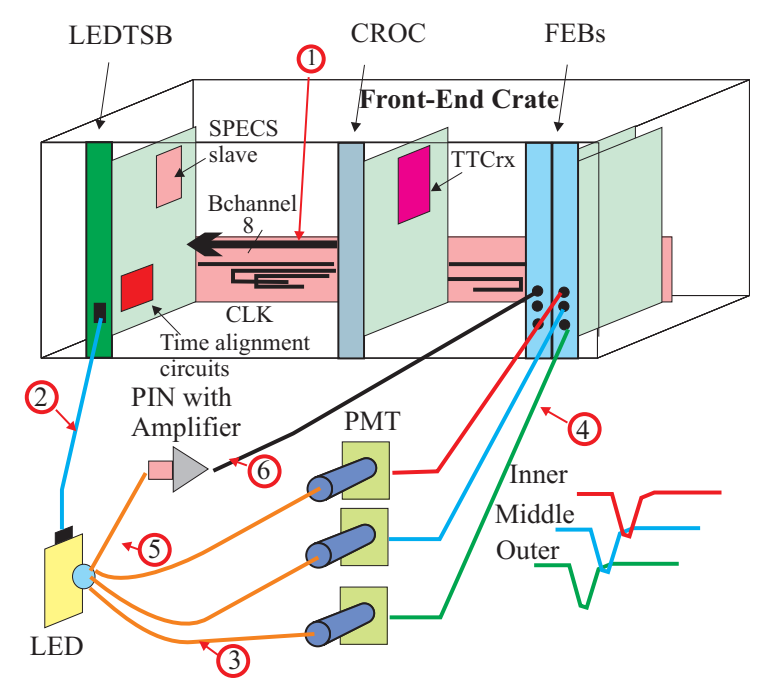

Figure 6.45: A sketch of the LED monitoring of the calorimeter.1: FE crate backplane over which the monitoring trigger request is transmitted, 2: LED trigger pulse cable, 3: Clear fibre to cell, 4: PMT signal cable, 5: Clear fibre to PIN diode, 6: PIN signal cable.

Each LED illuminates 9 or 16 cells via clear fibres routing the light to a connector at the front of each cell, and from there via a second clear fibre traversing the module and conducting the light directly through the light mixer to the PMT. Each LED also illuminates a PIN diode for monitoring the stability of the LED light output. There are 456 LEDs, each controlled by the LED driver, and 124 PIN diodes so that the LEDs sharing a PIN can be fired at different times.

Each HCAL module is equipped with two LEDs each illuminating every cell in the module. Since the PMTs in a module have different gains, which depend on the average momentum of the particles they are detecting, the light output of the two LEDs is set in the ratio of 1:4. Clear fibres bring the light of both LEDs to each PMT via its light mixer. One PIN diode monitors the stability of each LED. The 2 LEDs in each of the 52 modules illuminate 16 or 40 cells and are controlled by 104 LED drivers.

A LED monitoring sequence is initiated by the Readout Supervisor (RS) upon request and transmitted to the LEDTSBs via the CROCs and the FE crate backplane. The LEDTSBs are located in the front-end crates on the calorimeter platforms and each controls 64 LED drivers. A total of 686 LED drivers in the system therefore require 12 LEDTSBs. A correspondence between LEDs or groups of LEDs fired by each LEDTSB is preprogrammed in the LEDTSB FPGA. This allows a different pattern of the 64 drivers to be fired in each of 64 consecutive triggering sequences, thus providing maximum flexibility. The LED firing time is adjusted in the LEDTSB in steps of $1 \mathrm{~ns}$ so as to arrive at the first cell of the readout pipeline, 16 clock cycles ( $400 \mathrm{~ns})$ after the RS pulse. It emerges $4 \mu$ s later from the pipeline in time for the calorimeter trigger.

The LED trigger boards are decoupled with air transformer, are edge triggered, and use fast on board pulse shapers. They provide a pulse of less than $25 \mathrm{~ns}$ to fit within an LHC bucket and with a shape compatible with that generated by a particle.

The light intensity of the LEDs is controlled via ECS by the HV-LED-DAC boards which are located in areas of low radiation. A wide range of intensities is implemented to monitor the linearity of the PMTs. 
For each LED monitoring sequence a subset of each of the subdetectors is illuminated. A given group of channels is fired at a rate of $20-30 \mathrm{~Hz}$, resulting in an overall monitoring rate of 1 $\mathrm{kHz}$. For each trigger $3-5 \%$ of the channels contain data.

These data are read out and transferred to a monitoring farm, consisting of a dedicated node of the processing farm where they are analyzed to check the stability of the calorimeters. Should any channel be observed to be drifting in gain an alarm is generated. Depending on the type of alarm, the run is paused or a suitable correction to the HV computed and applied via the ECS system.

The LED system is also used to detect malfunctioning cells and bad cable connections. It also allows a first relative timing of the cells.

\subsection{Muon System}

Muon triggering and offline muon identification are fundamental requirements of the LHCb experiment. Muons are present in the final states of many CP-sensitive B decays, in particular the two gold-plated decays, $\mathrm{B}_{\mathrm{d}}^{0} \rightarrow \mathrm{J} / \psi\left(\mu^{+} \mu^{-}\right) \mathrm{K}_{\mathrm{S}}^{0}$ and $\mathrm{B}_{\mathrm{s}}^{0} \rightarrow \mathrm{J} / \psi\left(\mu^{+} \mu^{-}\right) \phi$ [2]. They play a major role in $\mathrm{CP}$ asymmetry and oscillation measurements, since muons from semi-leptonic $b$ decays provide a tag of the initial state flavor of the accompanying neutral B mesons. In addition, the study of rare $\mathrm{B}$ decays such as the flavour-changing neutral current decay, $\mathrm{B}_{\mathrm{s}}^{0} \rightarrow \mu^{+} \mu^{-}$, may reveal new physics beyond the Standard Model [163].

The muon system provides fast information for the high- $p_{\mathrm{T}}$ muon trigger at the earliest level (Level-0) and muon identification for the high-level trigger (HLT) and offline analysis.

\subsubsection{Overview}

The muon system [164-166], shown in figure 6.46, is composed of five stations (M1-M5) of rectangular shape, placed along the beam axis. The full system comprises 1380 chambers and covers a total area of $435 \mathrm{~m}^{2}$. The inner and outer angular acceptances of the muon system are 20 (16) $\mathrm{mrad}$ and 306 (258) mrad in the bending (non-bending) plane respectively. This results in an acceptance of about $20 \%$ for muons from inclusive $b$ semileptonic decays.

Stations M2 to M5 are placed downstream the calorimeters and are interleaved with iron absorbers $80 \mathrm{~cm}$ thick to select penetrating muons. The minimum momentum of a muon to cross the five stations is approximately $6 \mathrm{GeV} / c$ since the total absorber thickness, including the calorimeters, is approximately 20 interaction lengths. Station M1 is placed in front of the calorimeters and is used to improve the $p_{\mathrm{T}}$ measurement in the trigger. The geometry of the five stations is projective, meaning that all their transverse dimensions scale with the distance from the interaction point.

The detectors provide space point measurements of the tracks, providing binary (yes/no) information to the trigger processor (see section 7.1) and to the DAQ. The information is obtained by partitioning the detector into rectangular logical pads whose dimensions define the $x, y$ resolution.

The muon trigger is based on stand-alone muon track reconstruction and $p_{\mathrm{T}}$ measurement and requires aligned hits in all five stations. Stations M1-M3 have a high spatial resolution along the $x$ coordinate (bending plane). They are used to define the track direction and to calculate the $p_{\mathrm{T}}$ of the candidate muon with a resolution of $20 \%$. Stations M4 and M5 have a limited spatial resolution, their main purpose being the identification of penetrating particles. 


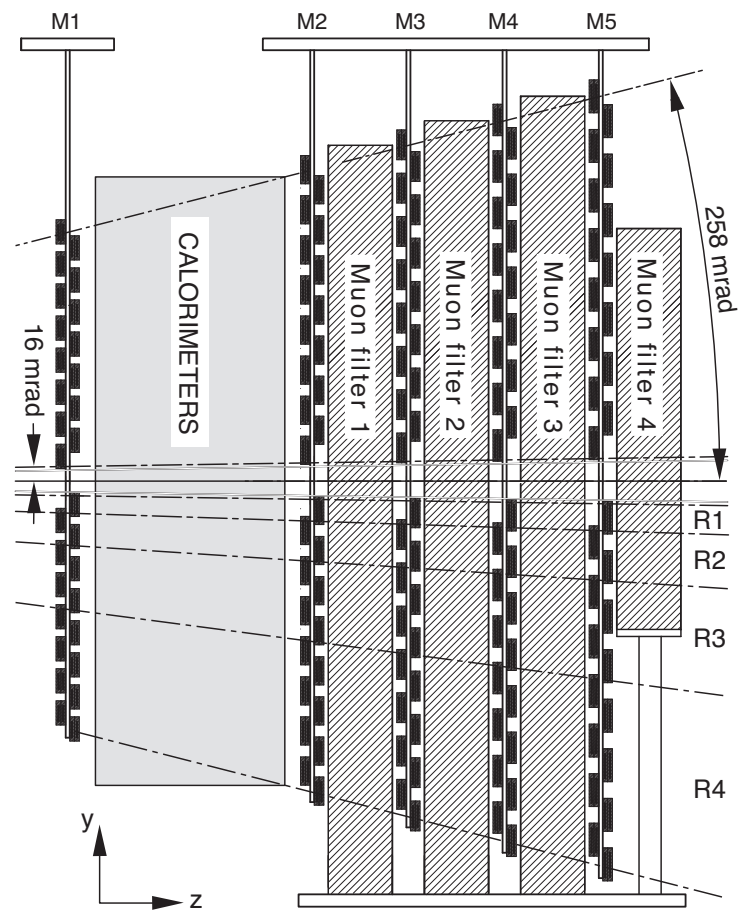

Figure 6.46: Side view of the muon system.

Appropriate programming of the L0 processing unit (see section 7.1.2) allows the muon trigger to operate in the absence of one station (M1, M4 or M5) or with missing chamber parts, although with degraded performance (worse $p_{\mathrm{T}}$ resolution).

The layout of the muon stations is shown in figure 6.47. Each Muon Station is divided into four regions, R1 to R4 with increasing distance from the beam axis. The linear dimensions of the regions $\mathrm{R} 1, \mathrm{R} 2, \mathrm{R} 3, \mathrm{R} 4$, and their segmentations scale in the ratio 1:2:4:8. With this geometry, the particle flux and channel occupancy are expected to be roughly the same over the four regions of a given station. The $(x, y)$ spatial resolution worsens far from the beam axis, where it is in any case limited by the increase of multiple scattering at large angles. The right part of figure 6.47 shows schematically the partitioning of the station M1 into logical pads and the $(x, y)$ granularity. Table 6.5 gives detailed information on the geometry of the muon stations.

\section{Simulation}

A complete simulation of the muon system was performed using GEANT4. Starting from the energy deposits of charged particles in the sensitive volumes, the detector signals were created and digitized taking into account detector effects such as efficiency, cross-talk, and dead time as well as effects arising from pile-up and spill-over of events occurring in previous bunch crossings [167]. 


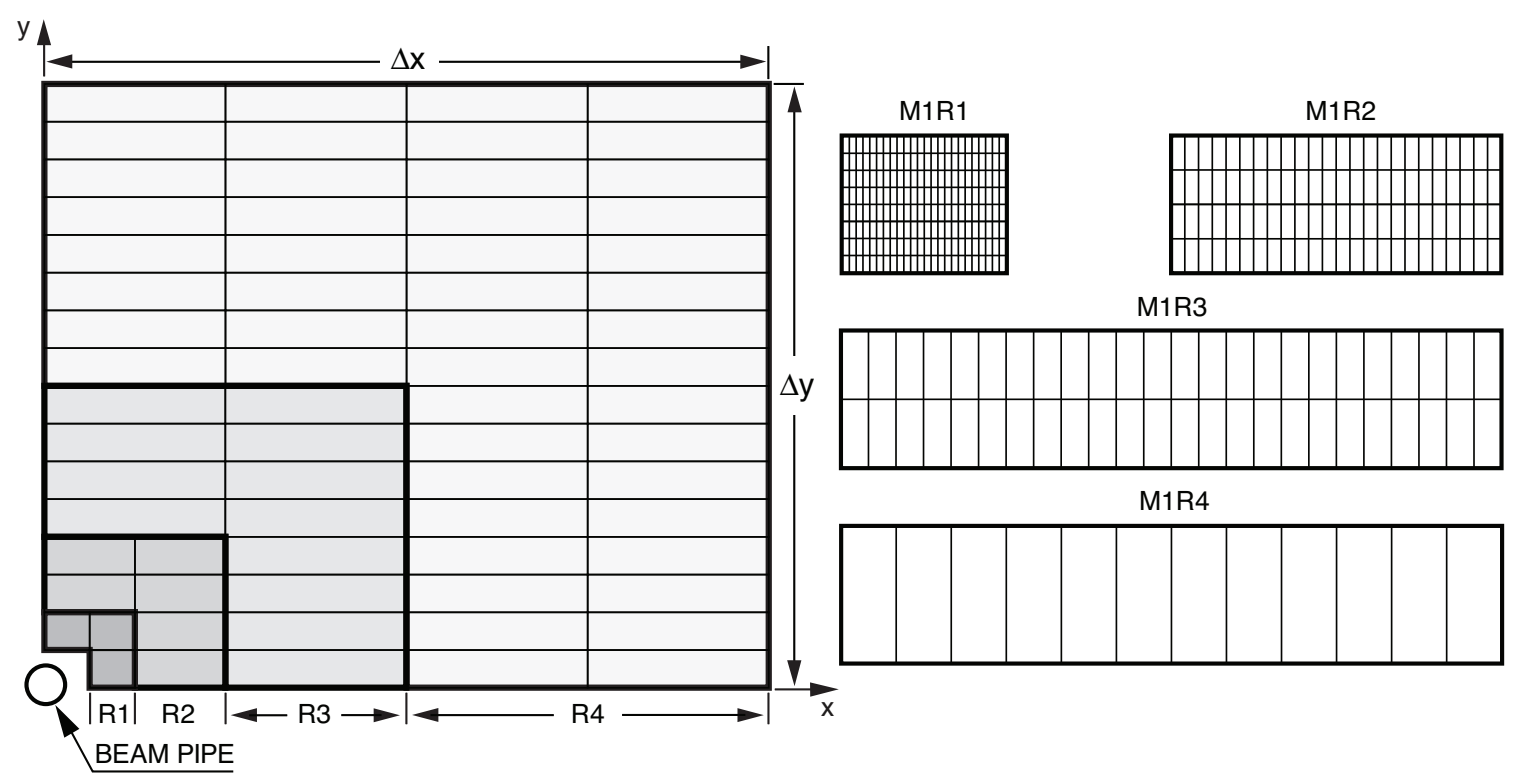

Figure 6.47: Left: front view of a quadrant of a muon station. Each rectangle represents one chamber. Each station contains 276 chambers. Right: division into logical pads of four chambers belonging to the four regions of station M1. In each region of stations M2-M3 (M4-M5) the number of pad columns per chamber is double (half) the number in the corresponding region of station M1, while the number of pad rows per chamber is the same (see table 6.5).

A realistic simulation of the detector occupancy requires the detailed description of the cavern geometry and of the beam line elements and the use of very low energy thresholds in GEANT4. The CPU time needed for such a simulation would be prohibitive for the stations M2-M5 interleaved with iron filters. The strategy chosen to overcome this problem was therefore to generate once for all a high statistics run of minimum bias events with low thresholds. The distributions of hit multiplicities obtained were parametrized and then used to statistically add hits to the standard LHCb simulated events. The latter were obtained by running GEANT4 at higher thresholds and with a simplified geometry of the cavern and the beam line [168]. Simulated events have been extensively used to evaluate the rates in the various detector regions in order to establish the required rate capabilities and ageing properties of the chambers and to evaluate the data flow through the DAQ system [169]. At a luminosity of $2 \times 10^{32} \mathrm{~cm}^{-2} \mathrm{~s}^{-1}$ the highest rates expected in the inner regions of M1 and M2 are respectively $80 \mathrm{kHz} / \mathrm{cm}^{2}$ and $13 \mathrm{kHz} / \mathrm{cm}^{2}$ per detector plane. In the detector design studies, a safety factor of 2 was applied to the M1 hit multiplicity and the low energy background in stations M2-M5 has been conservatively multiplied by a factor of 5 to account for uncertainties in the simulation.

\section{Detector technology}

The LHC bunch crossing rate of $40 \mathrm{MHz}$ and the intense flux of particles in the muon system [169] impose stringent requirements on the efficiency, time resolution, rate capability and ageing characteristics of the detectors, as well as on the speed and radiation resistance of the electronics. 
Table 6.5: Basic information for the five stations M1-M5 and the four regions R1-R4. All dimensions in $\mathrm{cm} . z$ : distance of the stations from the IP; $\Delta x$ and $\Delta y$ : dimensions of a quadrant in each station (see figure 6.47). Rows R1-R4: granularity of the different regions of the muon detector as seen by trigger and DAQ. Number of logical pads per chamber (in brackets) and size of the logical pads, along $x$ and $y$. In parentheses: size of the logical pads projected onto station M1.

\begin{tabular}{|c|c|c|c|c|c|}
\hline & M1 & M2 & M3 & M4 & M5 \\
\hline$z$ & 1210 & 1527 & 1647 & 1767 & 1887 \\
$\Delta x$ & 384 & 480 & 518 & 556 & 594 \\
$\Delta y$ & 320 & 400 & 432 & 464 & 495 \\
\hline & {$[24 \times 8]$} & {$[48 \times 8]$} & {$[48 \times 8]$} & {$[12 \times 8]$} & {$[12 \times 8]$} \\
R1 & $1 \times 2.5$ & $0.63 \times 3.1$ & $0.67 \times 3.4$ & $2.9 \times 3.6$ & $3.1 \times 3.9$ \\
& & $(0.5 \times 2.5)$ & $(0.5 \times 2.5)$ & $(2 \times 2.5)$ & $(2 \times 2.5)$ \\
\hline \multirow{3}{*}{ R2 } & {$[24 \times 4]$} & {$[48 \times 4]$} & {$[48 \times 4]$} & {$[12 \times 4]$} & {$[12 \times 4]$} \\
& $2 \times 5$ & $1.25 \times 6.3$ & $1.35 \times 6.8$ & $5.8 \times 7.3$ & $6.2 \times 7.7$ \\
& & $(1 \times 5)$ & $(1 \times 5)$ & $(4 \times 5)$ & $(4 \times 5)$ \\
\hline & {$[24 \times 2]$} & {$[48 \times 2]$} & {$[48 \times 2]$} & {$[12 \times 2]$} & {$[12 \times 2]$} \\
R3 & $4 \times 10$ & $2.5 \times 12.5$ & $2.7 \times 13.5$ & $11.6 \times 14.5$ & $12.4 \times 15.5$ \\
& & $(2 \times 10)$ & $(2 \times 10)$ & $(8 \times 10)$ & $(8 \times 10)$ \\
\hline & {$[12 \times 1]$} & {$[24 \times 1]$} & {$[24 \times 1]$} & {$[6 \times 1]$} & {$[6 \times 1]$} \\
R4 & $8 \times 20$ & $5 \times 25$ & $5.4 \times 27$ & $23.1 \times 29$ & $24.8 \times 30.9$ \\
& & $(4 \times 20)$ & $(4 \times 20)$ & $(16 \times 20)$ & $(16 \times 20)$ \\
\hline
\end{tabular}

Multi-wire proportional chambers (MWPC) are used for all regions except the inner region of station M1 where the expected particle rate exceeds safety limits for ageing. In this region triple-GEM detectors are used [166].

The trigger algorithm requires a five-fold coincidence between all the stations, therefore the efficiency of each station must be high enough to obtain a trigger efficiency of at least $95 \%$, within a time window smaller than $25 \mathrm{~ns}$ in order to unambiguously identify the bunch crossing. ${ }^{24}$ The necessary time resolution is ensured by a fast gas mixture and an optimized charge-collection geometry both for the MWPC and the GEM detectors. Moreover, the chambers are composed of four or two OR-ed gas gaps depending on station. In stations M2-M5 the MWPCs are composed of four gas gaps arranged in two sensitive layers with independent readout. In station M1 the chambers have only two gas gaps to minimize the material in front of the electromagnetic calorimeter. In region M1R1 two superimposed GEM chambers connected in OR are used.

To simplify the synchronization procedure and improve time alignment, the readout electronics is equipped with a 4-bit TDC which allows a fine time measurement of the signals with respect to the $25 \mathrm{~ns}$ machine clock. The fine time tuning is perfomed by selecting the hits belonging to penetrating tracks.

In addition, the use of two layers with independent HV supplies and the flexibility of the readout provide a high degree of redundancy built into the system.

\footnotetext{
${ }^{24}$ In the following, the system has been characterized assuming a conservative $20 \mathrm{~ns}$ window.
} 
Table 6.6: Readout methods used in the muon chambers.

\begin{tabular}{|l|l|}
\hline Readout type & Region \\
\hline \hline MWPC & \\
Wire pads & R4 \\
Mixed wire-cathode pads & R1-R2 in M2-M3 \\
Cathode pads & everywhere else \\
\hline GEM & \\
Anode pads & M1R1 \\
\hline
\end{tabular}

\section{Readout}

To satisfy the requirements of spatial resolution and rate capability that vary strongly over the detectors, different technical solutions are employed for the MWPC in different stations and regions.

All the chambers are segmented into physical pads: anode wire pads or cathode pads in the MWPCs and anode pads in the GEM chambers. Each physical pad is read out by one front-end (FE) electronics channel. The FE electronics is based on custom radiation-hard chips especially developed for the muon system. The input stage can be wired to handle either signal polarity: negative for anode pads, positive for cathode pads.

The electronics includes flexible logical units performing the OR of a variable number of FE channels following the requirements of the readout. The readout methods used in different detector regions are summarized in table 6.6.

The R4 regions contain most of the chambers and the spatial resolution required there is relatively modest. Therefore the simplest and safest technology was adopted: the physical pads are a group of adjacent wires connected together to the same FE channel. The length of the anode wires defines the spatial $y$ resolution, all the wires being aligned vertically. The requirement on the resolution limits the vertical size of the chambers to $20--30 \mathrm{~cm}$, and results in a large number of chambers.

Cathode pads (anode pads for GEM) are obtained by etching the desired pattern in the cathode (anode) planes. As can be seen from figure 6.47 all pads in R3 chambers can be accessed directly from the upper and lower sides. On the other hand, the chambers belonging to R1 and $\mathrm{R} 2$ have a chessboard pad structure so that a multilayer printed circuit board is used to bring the signals outside.

To keep the noise and the dead-time of the FE channels to an acceptable level, the rate of a given pad and its electrical capacitance must be limited. This implies that in most chambers the size of the physical pads (either wire or cathode pads) must be smaller than required by spatial resolution. In these cases up to four adjacent physical pads are OR-ed by the FE electronics to build a logical pad. However, in regions R1-R2 of stations M2-M3 the required spatial resolution in $x$ imposes logical pads which are too small to be practically built. For those chambers a mixed readout has been adopted: a narrow wire-strip to define the $x$ resolution, and a larger cathode pad to define the $y$ resolution, together defining are the logical channels seen by the trigger and DAQ. Logical pads are then obtained as an AND between wire and cathode pads (figure 6.48). 


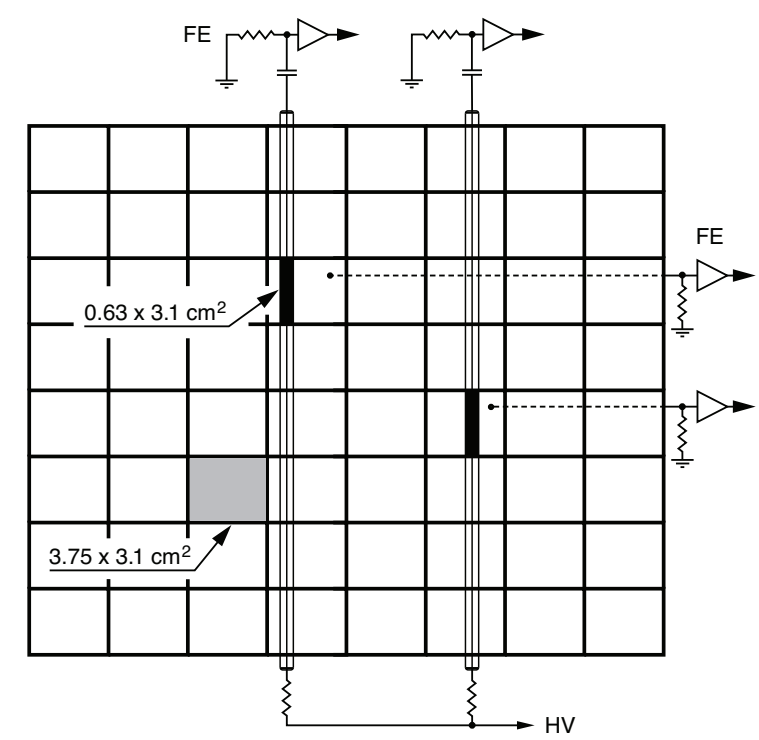

Figure 6.48: Scheme of the mixed wire-cathode pads readout in one M2R1 chamber. Two wirepad and two cathode-pad readout channels are shown. The coincidence between crossing vertical wire-pads and the cathode physical pads defines the logical pads, shown in black.

In the M1 station, where the foreseen channel occupancy is high, the signals from the logical pads are sent directly to the trigger and DAQ. In most of the other regions, in order to reduce the number of output optical fibres, several contiguous logical pads are further OR-ed to build larger logical channels in the form of vertical and horizontal strips. The logical pads are then reconstructed by the coincidence of two crossing strips. This operation is performed in the Level-0 Trigger Processor (see 7.1) and in the DAQ TELL1 boards (see 8.2).

Figure 6.49 shows the partitioning of a quadrant of stations M2 and M3 into sectors containing the crossing strips. The sector size is adapted to the trigger processing elements that work on a fixed number of logical pads belonging to a projective tower over the five stations.

The full muon system comprises 122112 physical channels ORed into 25920 logical channels which are transmitted via optical links to the Level-0 trigger and DAQ electronics. Appropriate combinations of logical channels in the Level-0 and High-Level Trigger provide the 55296 logical pads used for the muon tracking.

The specifications of the MWPCs and GEMs and their performance are summarised in the following sections.

\subsubsection{Wire chambers}

\section{Design}

The LHCb muon system comprises 1368 Multi Wire Proportional Chambers. Prototype studies [170-176] showed that a time resolution of about $5 \mathrm{~ns}$ can be achieved in a gas gap with a wire plane of $2 \mathrm{~mm}$ spacing, symmetrically placed in a $5 \mathrm{~mm}$ gas gap, using fast, non-flammable, gas mixtures of $\mathrm{Ar} / \mathrm{CO}_{2} / \mathrm{CF}_{4}$ with $40 \% \mathrm{Ar}$ and variable concentrations of $\mathrm{CO}_{2} / \mathrm{CF}_{4}$. Finally the 


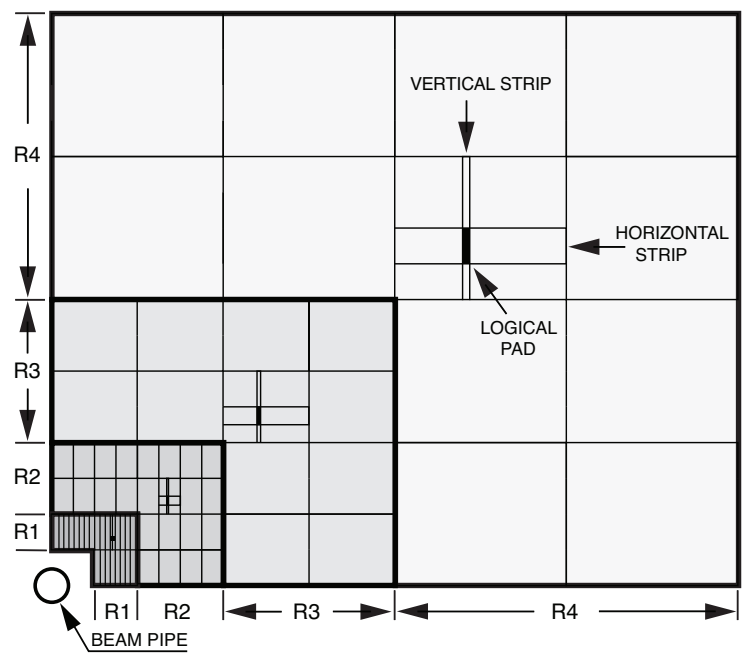

Figure 6.49: Front view of one quadrant of stations $M 2$ and $M 3$ showing the partitioning into sectors. In one sector of each region a horizontal and a vertical strip are shown. The intersection of a horizontal and a vertical strip defines a logical pad (see text). A Sector of region R1 (R2, R3, R4) contains $8(4,4,4)$ horizontal strips and $6(12,24,24)$ vertical strips.

Table 6.7: Main MWPC parameters.

\begin{tabular}{|l|l|}
\hline Parameter & Design value \\
\hline \hline No. of gaps & $4(2$ in M1) \\
Gas gap thickness & $5 \mathrm{~mm}$ \\
Anode-cathode spacing & $2.5 \mathrm{~mm}$ \\
Wire & Gold-plated Tungsten $30 \mu$ m diameter \\
Wire spacing & $2.0 \mathrm{~mm}$ \\
Wire length & 250 to $310 \mathrm{~mm}$ \\
Wire mechanical tension & $0.7 \mathrm{~N}$ \\
Total no. of wires & $\approx 3 \cdot 10^{6}$ \\
Operating voltage & $2.5-2.8 \mathrm{kV}$ \\
Gas mixture & $\mathrm{Ar} / \mathrm{CO}_{2} / \mathrm{CF}_{4}(40: 55: 5)$ \\
Primary ionisation & $\simeq 70 \mathrm{e}^{-} / \mathrm{cm}^{-}$ \\
Gas Gain & $\simeq 10^{5} @ 2.65 \mathrm{kV}$ \\
Gain uniformity & $\pm 20 \%$ typical \\
Charge/MIP (one gap) & $\simeq 0.6 \mathrm{pC} \mathrm{@} 2.65 \mathrm{kV}$ \\
\hline
\end{tabular}

mixture $\mathrm{Ar} / \mathrm{CO}_{2} / \mathrm{CF}_{4}(40: 55: 5)$ was adopted. By OR-ing the signals from two adjacent gas gaps the resulting double gap has an efficiency better than $95 \%$ in a $20 \mathrm{~ns}$ window at a gas of $G \simeq 10^{5}$. This gain is achieved at a voltage of 2600-2700 V [177]. Prototype tests with intense beams $\left(100 \mathrm{kHz} / \mathrm{cm}^{2}\right)$ confirmed the prediction that space-charge effects are negligible at the rates expected for the experiment [178].

The main parameters of the MWPC detectors are summarized in table 6.7. Detailed simulations [179] based on GARFIELD [180] were performed to optimize the design and to establish 


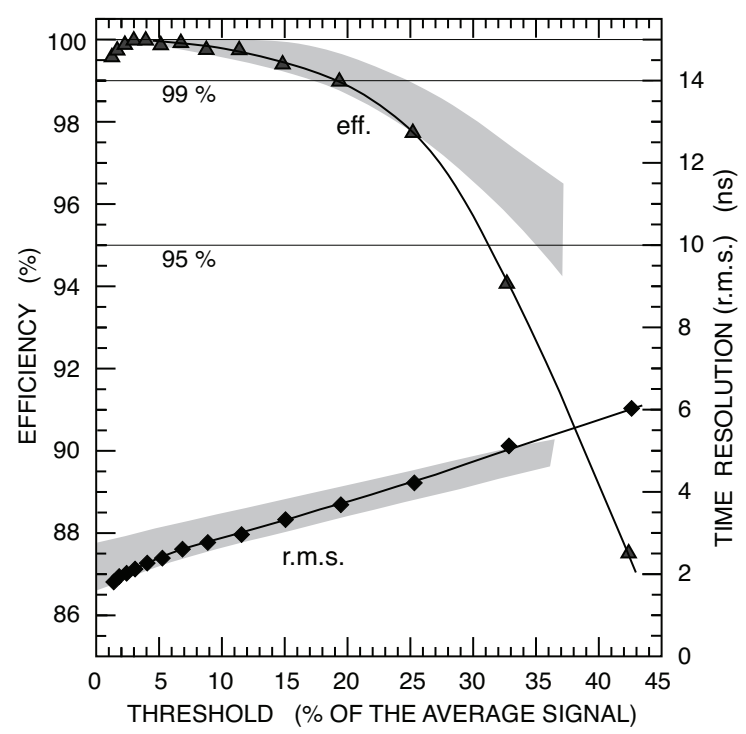

Figure 6.50: MWPC double-gap efficiency and time resolution vs. discriminator threshold for a prototype chamber. Solid lines: experimental results. The MonteCarlo simulation is shown in grey; the band accounts for different track angles.

the geometrical tolerances for the chamber construction. The primary ionisation was simulated by HEED [181] and drift and diffusion by MAGBOLTZ [182]. The design of the pad readout was optimized with SPICE [69] in order to keep the cross-talk between pads below 5\%. The maximal deviations of the construction parameters was established under the assumption of a maximum variation of gas gain of $\pm 20 \%$.

Figure 6.50 shows the good agreement of a MonteCarlo simulation for double-gap efficiency and time resolution versus FE threshold and the values measured on for a chamber prototype.

In stations M2-M5 a chamber is made of four equal gas gaps superimposed and rigidly stacked together with the gas flowing serially through all the gaps. Two contiguous gas gaps have their corresponding readout electrodes (either wire or cathode pads) hard-wired together in OR to form a double gap layer. The readout electrodes of the resulting layer are in turn connected to separate FE channels. As already mentioned, the M1 chambers contain only two gas gaps. These two gaps form the two layers which are readout independently. In order to maximize operation flexibility each gap has its own HV line and can be powered or switched off independently of the others.

Figure 6.51 shows an exploded schematic view of a chamber. The structural elements of the chamber are the panels, $9 \mathrm{~mm}$ thick, made of an insulating core sandwiched between two conducting planes. The conducting planes inside the chamber form the cathodes while the two external planes are grounded and act as an electrical shield. The panels are stacked using $2.5 \mathrm{~mm}$ thick PCB bars glued to the panels and superimposed to create the $5 \mathrm{~mm}$ gas gap. The wires are soldered and glued to the wire fixation bars while other bars seal the gap along the periphery. The $5 \mathrm{~mm}$ gap is ensured by several precision dowels inserted in the bars.

A cross section of a chamber is shown in figure 6.52. A U-shaped brass channel running around the chamber edges is soldered to the outer conductive planes to complete the chamber Faraday cage. The front-end boards, the LV voltage regulators and the HV filters are mounted 


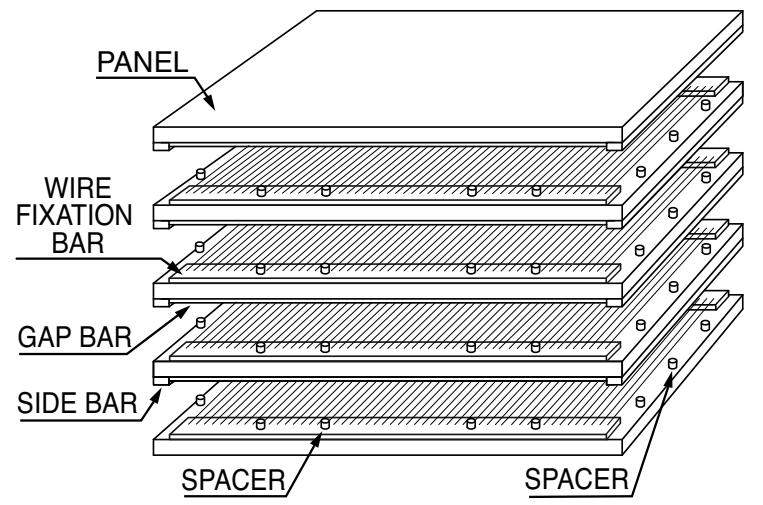

Figure 6.51: Exploded schematic view of a chamber showing the various elements.

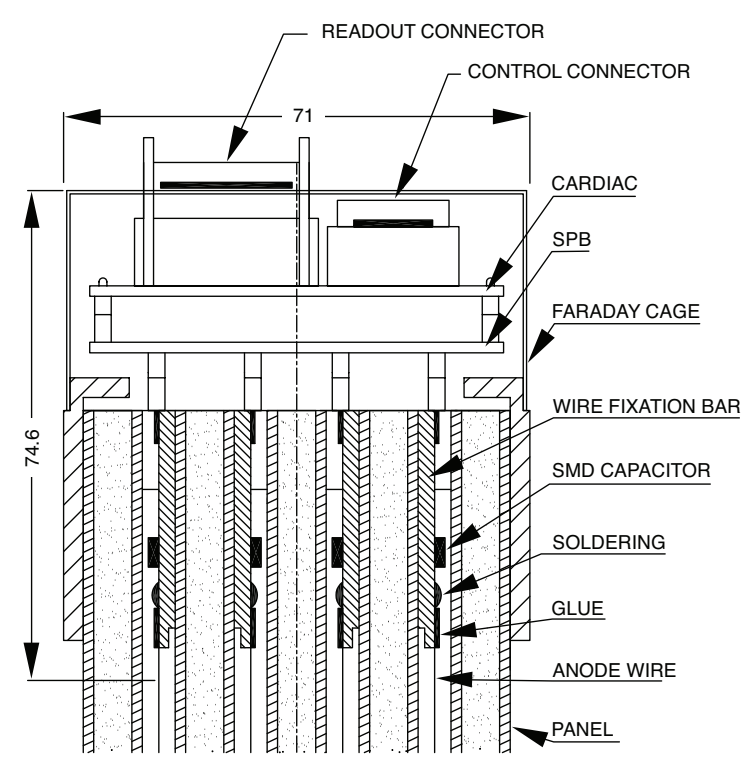

Figure 6.52: Cross section of a wire chamber showing the four gas gaps and the connection to the readout electronics. SPB: Spark Protection Board; CARDIAC: FE Electronics Board. In this case the hardwired OR forming the two Double Gaps (see text) is achieved in the SPB.

inside the Faraday cage to minimize electrical pickup. The HV is brought in through a custommade multipin connector and multiconductor cable. LVDS shielded cables are used for signal transmission and control.

The general design and construction is the same for all chambers and is discussed in detail in [183].

\section{Chamber construction}

Given the large number of chambers, the production was distributed among six production sites. A great effort went into ensuring that all those sites had equivalent facilities and tooling, albeit with some flexibility. The same stringent quality criteria and test protocols were adopted throughout to ensure a constant quality of the produced chambers. 


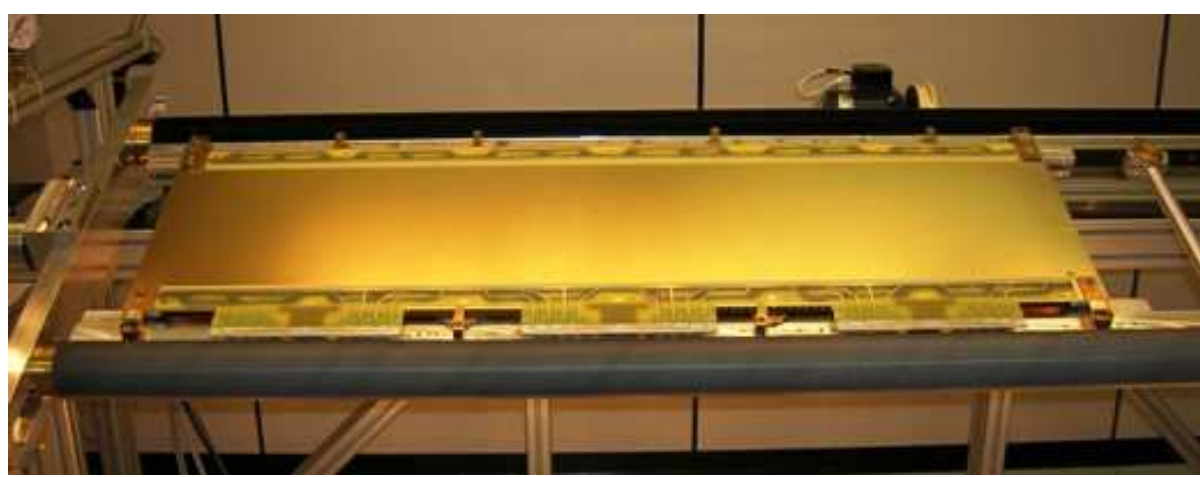

Figure 6.53: Panels mounted onto the winding machine frame ready to start wiring. The two combs which guide the wire are also visible. Below the panel in the foreground there is a second one attached to the underside of the frame. The two panels are wound simultaneously.

Panels The panels are the basis of the chamber mechanical structure. A panel consists of two copper clad fire-resistant fibreglass epoxy laminates (FR4), interleaved with a structural core. The panels are individually wired and then assembled to form the complete chamber.

The panel precision and its planarity define the gap quality. Therefore it is very important to achieve tight tolerances and stability in time, coupled with adequate robustness. In addition the panels must be light and stiff and easily adapted to mass production. The choice for the structural core was a polyurethane foam core for stations M2-M5 and a light honeycomb core for station M1 where a lower material budget is mandatory.

The panels were produced industrially. For the M2-M5 chambers precision machined molds were used to inject the polyurethane foam into the FR4 sandwich. Three molds of different sizes produced all the panels. For M1 chambers the core was made from a light NOMEX ${ }^{\circledR}$ sheet purchased with precise tolerance on thickness, and glued between the two FR4 foils. The pressure during gluing was assured by a vacuum bag. So the planarity of the panels is due to the quality of the honeycomb core and of the flatness of the assembly table.

The wire fixation bars (figure 6.51) were glued to the panels before the wiring process using an epoxy adhesive. ${ }^{25}$ The gluing was performed on a special table which ensured the exact height of the bars above the panel itself.

Wiring Specially built automatic winding machines were used in all the production sites to rapidly perform panel wiring. The machines could wind one or two panels simultaneously in about one hour for the largest chambers. The panels were fixed to a rigid frame, and grooved dowels (combs) were installed parallel to the panels long sides (figure 6.53). To achieve the required precision on wire pitch, the wire spacing was determined by the combs while the wire height with respect to the cathode-plane was adjusted by precision bars mounted to the frame. The winding machines were equipped with brake motors or electronic devices to keep the mechanical wire tension constant.

Once the winding was completed, the wires were glued and soldered. The wires were glued to the wire fixation bars using epoxy adhesive before soldering. This procedure guarantees that the

\footnotetext{
${ }^{25}$ Adekit A140 epoxy, Axon Technologies, USA.
} 


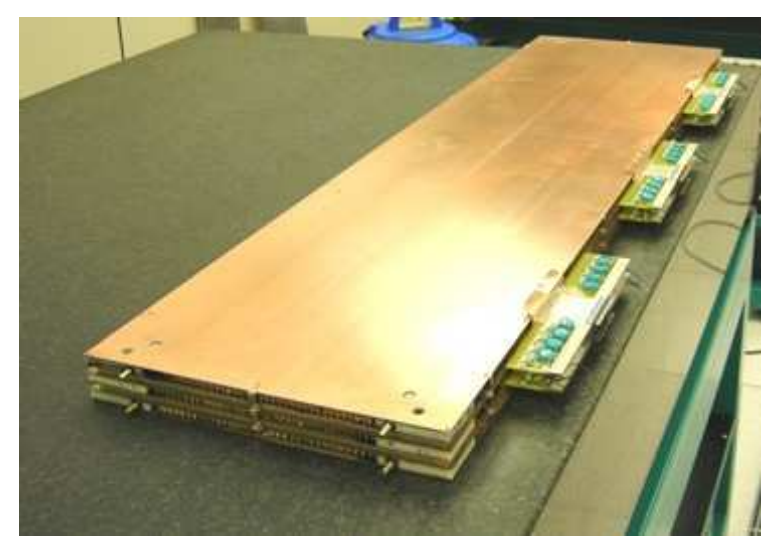

Figure 6.54: A M1R4 chamber completely assembled before the installation of the Faraday cage. The three honeycomb panels are visible. On the corners of the panels there are plastic inserts with internal channels for gas circulation. The gas nipples are inserted in the top and bottom panels. Decoupling HV capacitors, resistors and connectors for the FE boards are mounted on the printed cicuit boards on the right.

wires are kept in place with a fixed height with respect to the cathode plane. The gluing also keeps the wire tension to its nominal value.

Due to the large number of solder joints in the construction of the chambers, the use of an automated and reliable method was desirable. Automatic soldering stations [183] both with conventional soldering heads and with laser heads were employed.

Final assembly In the final assembly of the chamber the panels were superimposed and glued together using cylindrical precision spacers for alignment (see figure 6.51). The glue ${ }^{26}$ ensures the chamber gas tightness. In the chambers of region R1 in stations M2-M3 and of region R2 in stations M1-M3, no glue was used and gas tightness was ensured by O-rings made of natural rubber. This allows to open the chambers easily if needed. Screws were used to keep together the panels. Finally the brass channels forming the Faraday cage were soldered to the outer copper cladding of the panels. Figure 6.54 shows an assembled M1R4 without Faraday cage.

\section{Quality control and testing}

Uniform quality of the chambers produced in the various sites was ensured by stringent quality tests of the individual chamber components and of the assembled chambers [184-186]. All the panels were individully measured at production and then shipped to the production sites where they were checked once more before assembly. Despite the tight tolerances the yield of good panels was $90 \%$.

Once the panels were produced, the pitch and the mechanical tension were measured for all wires. The wire pitch was determined with an automated device based on two digital cameras [186]. The distribution of the measured wire pitch has an r.m.s. of $16 \mu \mathrm{m}$. Only a very small number of wires with pitch value outside tolerance $( \pm 0.1 \mathrm{~mm})$ had to be replaced.

\footnotetext{
${ }^{26}$ Adekit A145 and Araldite 2011 epoxy, Huntsman Advanced Materials, Switzerland.
} 


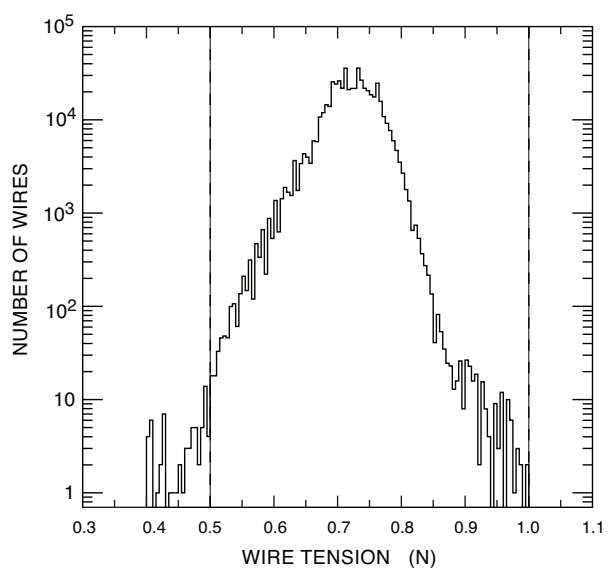

Figure 6.55: Distribution of the mechanical tensions of the wires as deduced from a measurement of the wire mechanical resonant frequency. The mean value of the tension is $0.73 \mathrm{~N}$ with an r.m.s. of $0.091 \mathrm{~N}$. The wires having a tension outside the interval $0.5-1 \mathrm{~N}$ (dashed lines) were replaced.

To determine the wire mechanical tension automated systems were developed $[187,188]$ which measures the mechanical resonant frequency of each wire. The distribution of the wire tension measured on the above mentioned sample is reported in figure 6.55.

The checks on the assembled chambers consisted of a gas leak test and a HV test at 2800 $2900 \mathrm{~V}$ using the standard gas mixture. The chamber gas leakage was determined by monitoring the decrease in time of an initial overpressure of 5 mbar. The method [186] had a sensitivity of about $0.01 \mathrm{mbar} /$ hour well below the maximum gas leakage rate accepted of $2 \mathrm{mbar} /$ hour.

The uniformity of the gas gain in the gaps was systematically checked using radioactive sources mounted on automated tables which performed an $(x, y)$ scan over the complete chamber surface $[185,186,189]$. Figure 6.56 shows the gain measurement on a sample of 184 chambers.

All the above checks were performed without the readout electronics installed.

Finally all chambers, fully equipped with the front-end electronics, underwent a final test with cosmic rays prior to installation and the electronic noise was checked once more when the chambers were mounted in the experiment. The maximum accepted noise rate was $1 \mathrm{kHz}$ per FE channel, a value which has no impact on the trigger system.

In addition, all the chambers for the regions exposed to the largest particle flux, were conditioned at the CERN Gamma Irradiation Facility (GIF) [190] to ensure their stable functionality under high radiation.

Important information about the individual components and the final chamber was stored in a database. This allows to retrieve at any time the results of all quality control measurements and will aid in understanding possible problems.

\section{Aging properties}

Extensive aging tests were performed on prototypes at the CERN GIF and at the Calliope ${ }^{27}$ facility. The goal of the tests was to prove that the performance of these chambers is not deteriorated by

\footnotetext{
${ }^{27}$ ENEA-Casaccia Research Centre, Rome.
} 


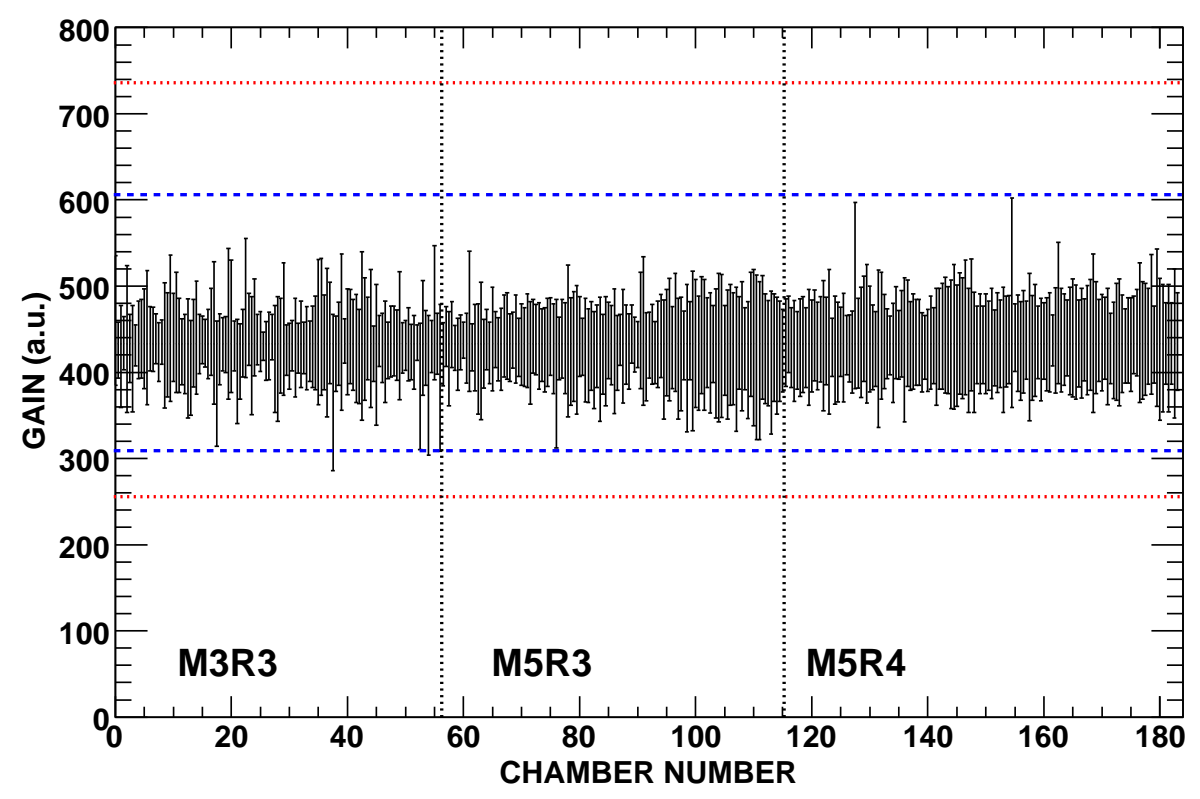

Figure 6.56: Gain uniformity measured for a sample of 184 chambers. The bars are proportional to the total gain spread of a double gap. The horizontal lines indicate the allowed acceptance intervals for good (in blue) and spare (in red) chambers.

the large radiation dose expected in the experiment in ten years of operation $\left(10^{8} \mathrm{~s}\right)$ at the nominal luminosity of $2 \times 10^{32} \mathrm{~cm}^{-2} \mathrm{~s}^{-1}$. In these tests the gain loss was measured against a non-irradiated reference chamber or gas gap.

In the test performed at the GIF facility at CERN, a four gap MWPC was irradiated for six months, accumulating a charge of $0.25 \mathrm{C} / \mathrm{cm}$ without detecting any gain loss [191].

In an accelerated test during 30 days of irradiation at the ${ }^{60} \mathrm{Co}$ source of Calliope, we monitored the currents in five four-gap MWPCs: four test chambers and a reference one [176, 192]. The chambers were operated at a voltage of $2.75 \mathrm{kV}$, corresponding to a gas gain of $1.8 \cdot 10^{5}$ [177], twice the value that will be adopted in the experiment. The largest integrated charge collected on a chamber wire was $0.44 \mathrm{C} / \mathrm{cm}$. This corresponds [169] to about 7 years $\left(10^{7} \mathrm{~s} /\right.$ year $)$ of operation of the most irradiated chamber (in M1R2) at a luminosity of $2 \times 10^{32} \mathrm{~cm}^{-2} \mathrm{~s}^{-1}$, assuming a safety factor of 2. No gain loss or other significant effect was detected.

\subsubsection{GEM chambers}

In the innermost region $\mathrm{R} 1$ of the station $\mathrm{M} 1$, the most stringent requirements for the detector is a rate capability of up to $500 \mathrm{kHz} / \mathrm{cm}^{2}$ of charged particles. Due to the large particle flux in this region the chambers must also be especially radiation hard such that no ageing effects will be visible in 10 years of LHCb operation. This is difficult to achieve in wire chambers unless the gain is decreased, at the price of a worse signal-to-noise ratio.

After an extensive R\&D program triple-GEM chambers with active area of $20 \times 24 \mathrm{~cm}^{2}$ were selected for M1R1. Each of the 12 chambers consists of two triple-GEM detectors superimposed and forming the two sensitive layers, which are then logically OR-ed. 


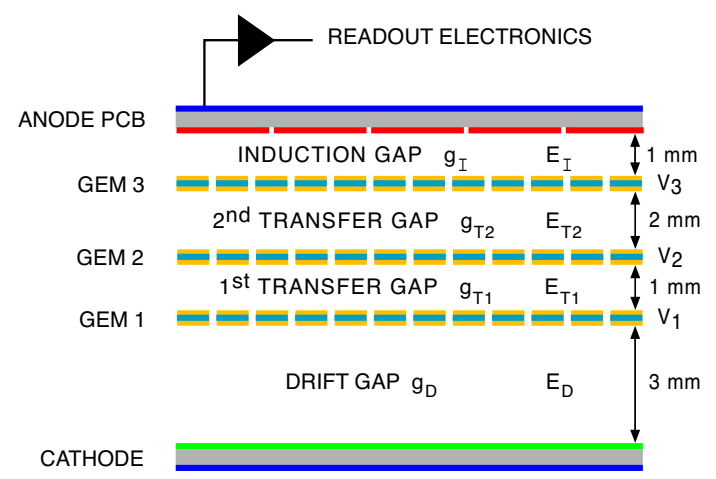

Figure 6.57: Schematic cross section of a triple-GEM detector showing the most relevant elements and dimensions (see text).

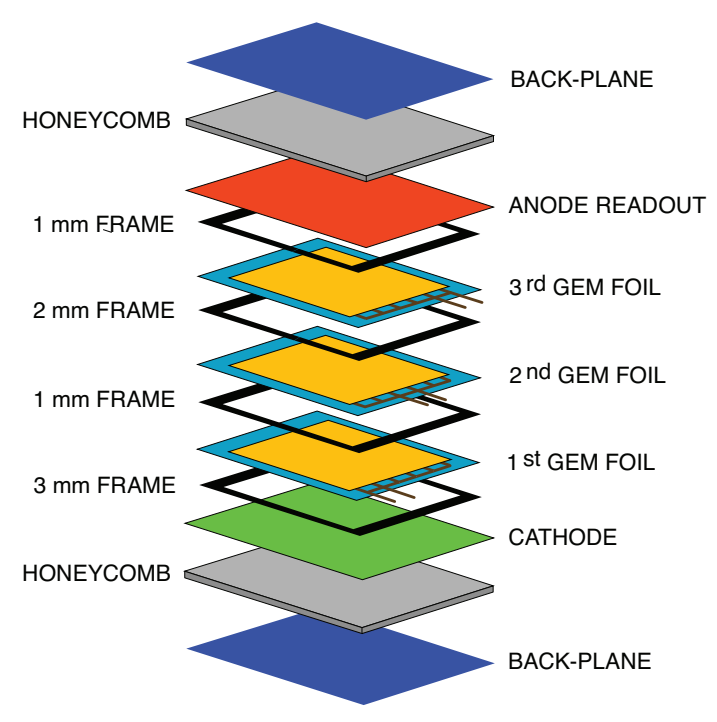

Figure 6.58: Exploded view of a triple-GEM detector.

\section{Design}

The triple-GEM detector, which consists of three gas electron multiplier (GEM) [193-195] foils sandwiched between anode and cathode planes, can effectively be used as tracking detector with good time and position resolution. A cross section of the detector, showing the different elements and their physical dimensions, is shown in figure 6.57. An exploded view is presented in figure 6.58 .

The ionisation electrons, produced in the drift gap between the cathode and the first GEM foil, are attracted by electric fields through the three GEM foils where they are multiplied. Once they cross the last GEM foil they drift to the anode in the induction gap, giving rise to an induced current signal on the pads.

Prototype tests have shown that the fast $\mathrm{Ar} / \mathrm{CO}_{2} / \mathrm{CF}_{4}(45: 15: 40)$ gas mixture allowed to achieve a time resolution better than $3 \mathrm{~ns}$, to be compared with the time resolution of $\sim 10 \mathrm{~ns}$ obtained with the standard $\mathrm{Ar} / \mathrm{CO}_{2}$ (70:30) gas mixture [196].

Another improvement in time performance has been obtained by optimizing the detector geometry. Mechanical considerations indicate that a minimum distance of $1 \mathrm{~mm}$ should be kept between GEM foils. The size of the drift gap $g_{D}$ is large enough to guarantee full efficiency for charged tracks. The first transfer gap $g_{T 1}$ is kept as small as possible to avoid that primary electrons produced in the same gap give rise to a signal over threshold. The second transfer gap $g_{T 2}$ is larger than the first one to let the diffusion spread the charge over more holes and then lower the discharge probability. The induction gap $g_{I}$ is kept as small as possible to maximize the signal fraction integrated by the amplifier.

The best values of the gap fields and of the voltage across the GEM foils were determined experimentally by optimizing time resolution versus discharge probability and are typically $E_{D}=$ $3.5 \mathrm{kV} / \mathrm{cm}, E_{T}=3.5 \mathrm{kV} / \mathrm{cm}$ and $E_{I}=5 \mathrm{kV} / \mathrm{cm}$ and $\mathrm{V}_{1}=440 \mathrm{~V}, \mathrm{~V}_{2}=430 \mathrm{~V}, \mathrm{~V}_{3}=410 \mathrm{~V}$. The anode pad printed circuit board is such that the pad to pad distance is $0.6 \mathrm{~mm}$ and the pads are surrounded by a ground grid of $0.2 \mathrm{~mm}$ thickness to suppress cross-talk. 


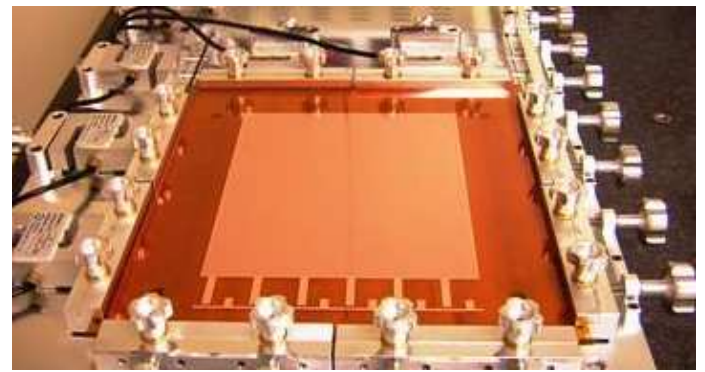

Figure 6.59: Stretching of a GEM foil.

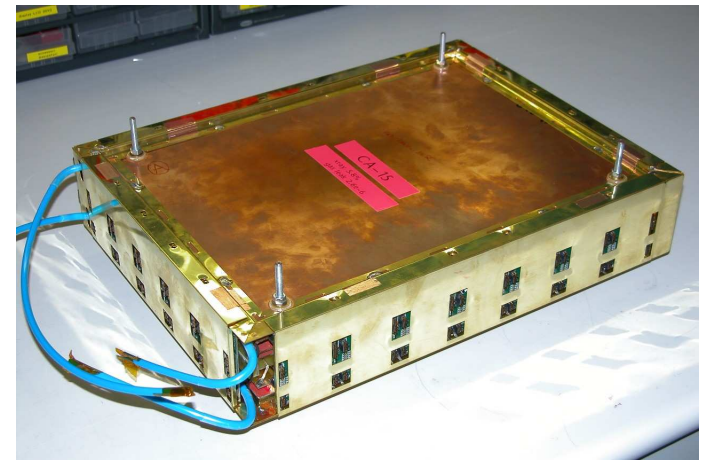

Figure 6.60: GEM chamber fully assembled with the $24 \mathrm{FE}$ electronics boards. The cutouts in the Faraday cage allow the insertion of the cable connectors.

Measurements on prototype chambers made of two detectors in OR showed an efficiency better than $96 \%$ in a $20 \mathrm{~ns}$ window at a gain of $6 \cdot 10^{3}$. The pad cluster size upper limit of 1.2 and the discharge studies suggested a maximum working gain of about $2 \cdot 10^{4}$ [195].

\section{Chamber construction}

All the critical steps in chamber assembly were carried out in class 1000 clean rooms or under class 1000 huts, with controlled temperature and humidity conditions. The huts were used for all operations involving GEM foils.

The GEM foils, $50 \mu \mathrm{m}$ thick Kapton with two-sided $\mathrm{Cu}$ cladding of $5 \mu \mathrm{m}$, have an active area of $202 \times 242 \mathrm{~mm}^{2}$ and were manufactured by the CERN-EST-DEM workshop. The holes have a bi-conical structure with external (internal) diameter of 70 (50) $\mu \mathrm{m}$ and a pitch of $140 \mu \mathrm{m}$. In order to limit the damage in case of discharge, one side of the GEM foil is divided into six sectors, of about $33 \mathrm{~mm} \times 240 \mathrm{~mm}$. The separation between sectors is $200 \mu \mathrm{m}$. Each individual foil was visually inspected for defects and for leakage current. Then the foils were stretched with a mechanical tension of about $1 \mathrm{~kg} / \mathrm{cm}$ with a special device visible in figure 6.59 .

After the GEM stretching, a fibreglass frame was glued on the GEM foil using a Ciba 2012 epoxy. Both cathode and readout pad electrodes, realized on standard $1.0 \mathrm{~mm}$ thick printed circuit board, were respectively coupled with a $1.0 \mathrm{~mm}$ fibreglass foil by means of an $8 \mathrm{~mm}$ thick honeycomb structure, The back-panel with a $12 \mu \mathrm{m}$ copper layer deposition on its external side is used as a Faraday cage for the detector. The stiff cathode and pad panels act as support plates for the whole detector. These panels house two gas inlets and two gas outlets, made with machined Stesalit inserts.

All fibreglass parts that are in contact with the sensitive volume of the detector, were visually inspected in order to eliminate any residual spikes or broken fibres. They were then cleaned in an ultrasonic bath with de-mineralized water and dried in an oven at a temperature of $80^{\circ} \mathrm{C}$ during one night. 


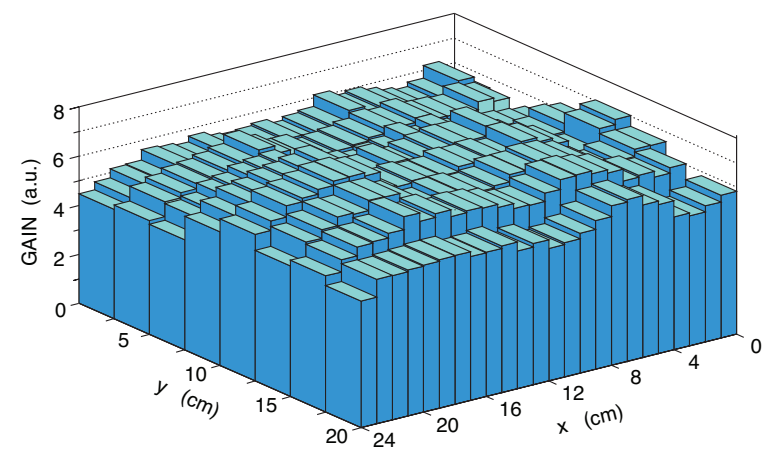

Figure 6.61: Response uniformity of a GEM chamber measured with an X-ray gun. The RMS gain variation over the $24 \times 8$ pads is typically less than $10 \%$.

The detectors were built on a high-precision reference plane by piling up and gluing together the component parts in the following order (see figure 6.58): cathode panel; the first GEM foil (GEM1) glued on a $3 \mathrm{~mm}$ thick fibreglass frame; the second GEM foil (GEM2) glued on a $1 \mathrm{~mm}$ thick frame; the third GEM foil (GEM3) glued on a $2 \mathrm{~mm}$ thick frame and then the last $1 \mathrm{~mm}$ thick frame followed by the pad panel. All the gluing operations were performed using Araldite AY103 epoxy and HY991 hardener. The aging properties of both glues have been studied during irradiation tests $[197,198]$. A complete chamber is shown in figure 6.60.

\section{Quality control and testing}

Several quality checks were performed on individual detector components before chamber assembly. Since the cathode and anode panels are the main mechanical structure of the detector, they were checked for planarity. Measurements over the whole panels showed that the deviation from the average plane was of the order of $50 \mu \mathrm{m}$ (r.m.s).

The quality of GEM foils was checked by performing various tests. A preliminary optical inspection was performed with a microscope to check for photolithographic imperfections. If the GEM foil passed the visual inspection, a high voltage test was performed. Such a test was done in a gas tight box, flushed with nitrogen too keep the relative humidity at $\sim 25 \%$ level. The foil was accepted if the leakage current was less than $1 \mathrm{nA}$ at $500 \mathrm{~V}$.

After construction, the detector gas leak rate was measured. This measurement was performed by inflating the detector to an overpressure of $\sim 10$ mbar together with another gas-tight chamber used as reference, and recording the overpressure decay. The typical gas leak rate of a detector is of the order of few mbar/day. At the gas flow forseen in the experiment $\left(80 \mathrm{~cm}^{3} / \mathrm{min}\right)$ this leakage rate will result in an acceptable humidity level lower than $100 \mathrm{ppm}$.

A gain uniformity test, performed with an X-ray gun, checked both the mechanical tolerance and the response uniformity of single detectors. A typical result is presented in figure 6.61. The current signals induced on each of the 192 pads of each detector were corrected for temperature and pressure variations. The current deviations from the average were all below $20 \%$ with a typical RMS $<10 \%$. Finally, a cosmic ray test was performed on all chambers fully equipped with frontend electronics. 


\section{Discharge and aging properties}

The use of the non-standard $\mathrm{Ar} / \mathrm{CO}_{2} / \mathrm{CF}_{4}(45: 15: 40)$ gas mixture required the demonstration of high robusteness of the GEM to discharges and ageing effects. In fact the choice of the electric field in the detector gaps and the unbalanced configuration of the voltages applied to the GEM foils $\left(\mathrm{V}_{1}>\mathrm{V}_{2}>\mathrm{V}_{3}\right.$, see figure 6.57) are the result of a minimization of discharges produced by alpha particles. More than 5000 discharges were integrated with neither breakdown nor performance losses using alpha particles and a high intensity low momentum pion/proton beam. These measurements demonstrated that GEMs in M1R1 can operate safely. The large fraction of $\mathrm{CF}_{4}$ in the gas required that a global irradiation test of the final chamber be performed to check the compatibility between the construction materials and the gas mixture. For this reason a test was performed at the Calliope facility with an intense gamma ray flux from a ${ }^{60} \mathrm{Co}$ source. In this test, a charge of 2.2 $\mathrm{C} / \mathrm{cm}^{2}$ on the anode pads and GEM3 foil was integrated. This is the charge foreseen for more than 10 years running if the GEMs are operated with a gain of $\sim 6000$ and a safety factor of 2 is applied. The performance of these chambers was measured in a test beam before and after irradiation and no damage or performance losses were detected [197, 198].

\subsubsection{Muon System mechanics}

All muon stations, including the iron filters, are separated into two halves which can move on rails away from the beampipe for maintenance and installation. The chambers, mounted on support walls, can also move with respect to the iron filters which are normally located close to the beampipe. Iron plugs minimize the free space between the beampipe and the iron filters to reduce small-angle background.

For M2-M5 two large support structures built from iron beams accomodate the suspensions for the four chamber support walls and have platforms for the electronics racks and gas systems. Cable chains are used to connect the cables, fibers, gas pipes and water cooling pipes with the outside. Station M1 has independent support structure and the related racks are located on the floor of the cavern. Cable chains are used in M1 to carry the 1200 cables to the racks.

The walls are designed to support the weight of the half-stations with minimal thickess and are built of aluminium sandwich plates. M1 wall is thinner to minimize material in front of the electromagnetic calorimeter. This is possible since the weight of M1 chambers is reduced thanks to the use of only two gaps and honeycomb panels. The material budget of the M1 station is 0.26 $\mathrm{X}_{0}$ on average with the chambers and the FE electronics contributing $0.16 \mathrm{X}_{0}$ and the mechanics, cables and gas pipes contributing the rest.

\footnotetext{
Alignment

The position of the chambers must be adjusted in order to minimize chamber overlaps which would introduce ambiguities. Moreover, since the muon trigger relies on the projectivity of the stations, a precise relative space alignment has to be performed, in particular in the inner region where an accuracy of about $1 \mathrm{~mm}$ is required.

The chambers are mounted with screws on angle brackets fixed to the supporting walls. The walls were first precisely aligned by adjusting the overhead suspensions, then each chamber was
} 


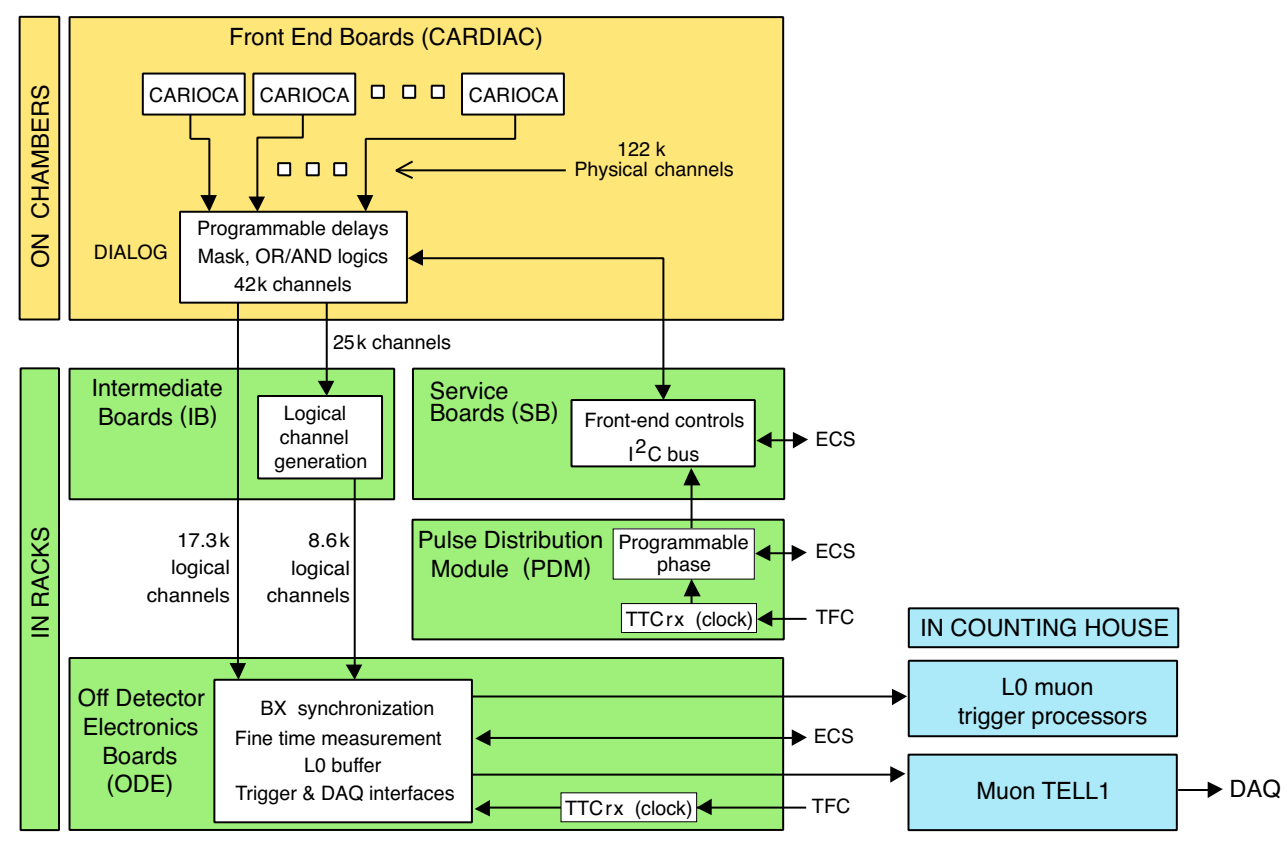

Figure 6.62: Simplified scheme of the Muon electronics architecture.

aligned relative to the wall. The position was adjusted vertically using spacers and horizontally via the slotted holes in the brackets. For horizontal and vertical alignment, the reference points were the support wall edge close to the beampipe and the top edge, respectively. Finally, the equipped walls were precisely aligned together using as reference each half-station, the centre of the beampipe. The reproducibility of the measurements is $\mathscr{O}(1) \mathrm{mm}$ and the reproducibility of the position after moving the walls is of the same order.

\subsubsection{Electronics}

Figure 6.62 shows schematically the architecture of the Muon readout electronics. The task of the electronics is twofold: prepare the information needed by the Level-0 muon trigger and send the data to the DAQ system. The main steps are:

i. the front-end CARDIAC boards perform the amplification, shaping and discrimination of the $\approx 120 \mathrm{k}$ chamber signals. The time alignment to within $1.6 \mathrm{~ns}$ of the different channels needed to correct for different cable lengths and different chamber behaviour is also done in this step. This is mandatory since the Muon Trigger is fully synchronous with is also done in this step, the LHC cycle.

ii. The $\approx 26 \mathrm{k}$ logical-channel signals are generated by suitable logical ORs of the physical channels. This step is performed on the FE boards and on special Intermediate Boards (IB), when the logical channel spans more than one FE board.

iii. The Off Detector Electronics (ODE) boards receive the signals from the logical channels. They are tagged with the number of the bunch crossing (BX identifier) and routed to the trigger processors via optical links without zero suppression. 
iv. The fine time information, measured by the TDC on the ODE boards, is added and the data are transmitted via optical links to the TELL1 board and from the TELL1 to the DAQ system.

As far as possible, step ii. is performed on the chambers front-end boards in order to minimize the number of LVDS links exiting the detector. The other steps are performed on dedicated electronics boards mounted on racks on the left and right sides of the muon stations M2-M5 which also accomodate the LV and HV power supplies. Since the racks are installed on the same structure which supports the stations, cable chains for the chambers could be avoided. For station M1 the electronics racks are placed under the RICH and, as mentioned above, are connected via cable chains to the chamber walls.

\section{Radiation issues}

The Muon electronics must operate reliably for more than 10 years in a hostile environment. The FE boards in the regions close to the beampipe are exposed to very high radiation doses: in M1 the maximum total ionising dose (TID) foreseen in 10 years is $5000 \mathrm{~Gy}$. This decreases by only a factor 3 for M2. The maximum neutron and hadron fluences are in the range of $10^{13} / \mathrm{cm}^{2}$. Therefore, all the ASICs used in the FE Electronics were produced using radiation-hard technology.

The radiation dose is considerably less on the detector periphery where the electronics racks are located. TID values of up to $10 \mathrm{~Gy}$ in 10 years are expected in the electronics racks. ${ }^{28}$ These doses nevertheless dictate the use of radiation tolerant components or a proper qualification of commercial components.

For complex logic design, two kinds of FPGA both from the ACTEL family are used. The A54SX family was chosen first for good radiation immunity and was used in the Intermediate Boards (IB). A more advanced type of FPGA from the flash-based ProASICPLUS APA family, was chosen in the design of ODE, PDM and SB boards. Based on ACTEL flash technology, ProASICPLUS devices offer reprogrammability and nonvolatility in a high density programmable logic product. The ProASICPLUS is also suitable in a high radiation environment thanks to the radiation hardness of its flash cells.

Commercial off-the-shelf electronics (COTS) was used for glue logic and signal conversion. All chip were validated with respect to the radiation environment.

\section{Dedicated ASICs}

The large number of channels in the muon system, the flexibility necessary to adapt the readout scheme to the different regions, the necessity to synchronize all the channels in a $20 \mathrm{~ns}$ window, the high radiation expected in M1 and in the inner regions led us to develop three dedicated rad-hard ASICs (CARIOCA, DIALOG and SYNC) using IBM 0.25 micron technology. All chips provide and accept logical LVDS data.

\section{Front-end board}

The chamber readout is performed via front-end boards (CARDIAC) [199] plugged directly onto the chambers. Each CARDIAC has 16 inputs and 8 outputs, and is equipped with two CARIOCA

\footnotetext{
${ }^{28}$ this value includes a safety factor of 2 .
} 

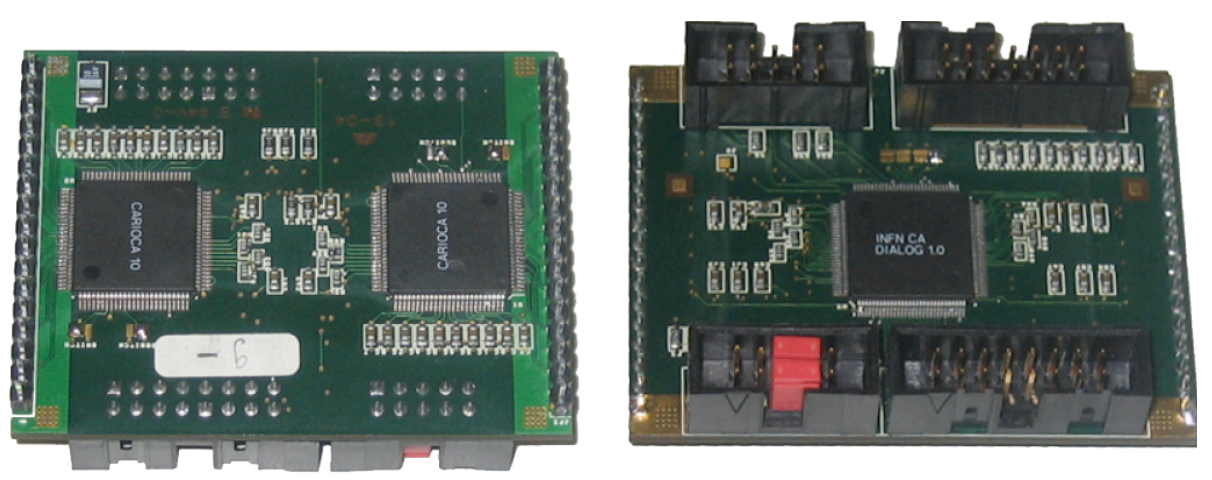

Figure 6.63: Top and bottom views of the CARDIAC board, showing the two CARIOCA chips and the DIALOG chip.

chips and one DIALOG chip, as shown in figure 6.63. A special diode circuit protects the front-end amplifiers from sparks and is mounted on a separated board.

The CARIOCA [200-202] is a front-end amplifier-shaper-discriminator chip with eight channels whose input polarity is selectable. The front-end current preamplifier can handle well the large spread in detector capacitances encountered in the muon chambers (from $20 \mathrm{pF}$ for the M1R1 to $220 \mathrm{pF}$ for the M5R4 chambers). The peaking time is $\sim 10 \mathrm{~ns}$ at the lowest detector capacitance. The input impedance is $50 \mathrm{Ohm}$ which is important to reduce internal pad-pad cross-talk. The chip includes tail cancellation and baseline restoring. The dead time of a fired channel, almost independent of the pulse height, is in the range 50-60 ns.

The CARIOCA has separate thresholds for all the channels in order to overcome the problem of channel-to-channel uniformity in the internal discriminators. Equivalent noise is about 2000 electrons at 0 capacitance, and increases as (42-45) e/pF. Power consumption is about $45 \mathrm{~mW} / \mathrm{ch}$.

The DIALOG $[203,204]$ chip has 16 inputs to receive the outputs from two CARIOCA chips, and performs the logical OR of corresponding pads in the two layers of a chamber to form logical channels. The DIALOG is equipped with adjustable delays for every input allowing the various input signals to be aligned with a step of $1.6 \mathrm{~ns}$. In addition, the DIALOG also allows setting the CARIOCA thresholds and can mask individual channels. It also contains features useful for system set-up, monitoring and debugging. Triple-voting and auto-corrected registers are used to increase single event upset immunity.

The CARDIAC boards are enclosed inside the chamber Faraday cage, together with radiationtolerant voltage regulators (LH4913) which supply the necessary $2.5 \mathrm{~V}$ to the boards. The R4 chambers use only three CARDIAC boards and one regulator each, while the M1R2 chambers require 24 CARDIAC boards and six regulators each.

Given the large number of readout channels the muon system comprises nearly 8000 CARDIACs. All the boards were tested after assembly, and had to pass successfully a thermal cycle to be accepted. Once mounted on the chamber the characteristics of each board, in particular with respect to noise, were measured again.

A special version of the CARIOCA chip, with lower threshold and longer shaping time because of the lower gas gain, CARIOCAGEM [202], has been produced for use on the GEMs. The tail cancellation is suppressed considering the purely electron signal in the GEM case. A more compact CARDIAC card has also been designed, given the tight space available in the R1 region. 


\section{SB and PDM boards}

The front-end boards are managed by the Service Boards (SB) [205] that handle the setting of the thresholds in the CARIOCA chip, as well as the adjustable delays, the channel masking and the setting of the AND/OR logics in the DIALOG chip. Each SB houses four ELMB [206] modules based on an 8-bit microcontroller (Atmel ATMega128) whose firmware was customized to permit $\mathrm{I}^{2} \mathrm{C}$ communication with the CARDIACs via twelve serial links. Each SB can control up to 192 CARDIAC boards. The full system comprises $156 \mathrm{SBs}$, managing the approximately $8000 \mathrm{CAR}-$ DIAC boards of the muon system. Most of the calibration and test procedures of the front-end are implemented in the ELMB firmware and are performed directly by the SBs.

A Pulse Distribution Module (PDM) resides in each of the 10 crates containing the service boards. The PDM is based on an ELMB and houses one TTCrx chip which generates low-jitter pulses in a chosen phase relation with the LHC machine clock. The pulses are then distributed to the service boards by means of a custom back plane. This facility is crucial for the time alignment of the muon system.

\section{IB board}

Whenever the generation of the logical channels is not possible at the DIALOG level, an additional logical layer is needed. This happens in regions R2, R3 and R4 of stations M2 to M5. The needed layer, the Intermediate Board (IB), implements the necessary logic on three ACTEL A54SX16A anti-fuse FPGAs. Anti-fuse technology offers good tolerance against high radiation doses. The IB has been tested successfully with up to 68.5 Gy without failures. The system comprises 168 IB boards which are installed in the electronics racks.

\section{ODE board}

The ODE board [207] contains the Level-0 pipelines and DAQ interface. It synchronizes signals and dispatches them to the Level-0 trigger. Each ODE receives synchronous TFC signals by way of a TTCrx chip [113]. On-board clock de-jittering and distribution is managed by a QPLL chip [208]. A total of 152 ODE installed on the same racks as the IBs, are used.

Each board receives up to 192 logical channels and outputs data to the Level-0 muon trigger and to the DAQ system. The incoming signals are assigned the appropriate BX identifier and are sent to the Level-0 muon trigger directly via twelve $1.6 \mathrm{~Gb} / \mathrm{s}$ optical links, each served by one GOL chip [209]. In parallel, the data are sent to the Level-0 pipelines, where they reside for $4 \mu$ s before receiving the LO-accept signal. Upon reception of a trigger, data are written into the LO derandomizer, a FIFO programmable to a maximum depth of 16 data words. The derandomizer allows the data to be read out at a regular rate of $1.1 \mathrm{MHz}$ with a safe margin with respect to the L0 average trigger rate of $1 \mathrm{MHz}$. Its programmable depth allows it to cope with instantaneous bursts of up to 16 consecutive triggers. Finally the data are formatted and sent to the TELL1 boards. The LVDS receivers, the Level-0 pipelines, the Level-0 derandomizer and the 4-bit TDC for fine time alignment are integrated into a single component, the SYNC chip [204]. The chip incorporates a number of error-detection features, allowing remote control and diagnosis of possible malfunctions on the boards. The other main board components (board controller, Level-0 buffer and DAQ interfaces) 
are based on one FPGA. ${ }^{29}$ Each ODE board also contains a CAN node, based on one Embedded Local Monitor Board (ELMB) [206], for board control via the ECS.

Channel mapping to the Level-0 trigger is organized by grouping the logical channels in three different ways. This is accomplished placing the SYNC chips on daughter-boards of three different sizes, containing 2, 4 or 6 chips, while the ODE mother board is always the same. In the three cases 12, 8 or 6 links respectively are active on the daughter board. The design of the ODE board has been very challenging because it must cope with several, and sometime contradictory, requirements. Due to the limited space available, the board has a high channel density (192 differential inputs into a $6 \mathrm{U}$ card), and should provide a good level of flexibility and at the same time match the different trigger sector topologies. High signal integrity is also mandatory to guarantee high quality optical transmission. For radiation tolerance, a triple modular redundancy technique has been used whenever possible to increase single event upset immunity.

\section{Muon TELL1 features}

Upon reception of a trigger, the data from the detector logical channels are transmitted from the ODE to the TELL1 and from the TELL1 to the DAQ system. The description of the TELL1 board is given in section 8.2. The muon-specific TELL1 board, performs standard control of event synchronization, as well as zero suppression and is also programmed to reconstruct the logical pads as the intersection of two crossing logical channels of the stations M2 to M5. This information is added to the event data to speed up the muon identification algorithm used in the HLT trigger. The muon system requires 14 TELL1 boards (four in the M1 and M2 stations and 2 in the M3, M4, and M5 stations). These numbers are determined by data flow and connectivity requirements as well as the required ECS partitioning.

\subsubsection{LV and HV systems}

The Low Voltage and High Voltage systems are based on radiation-tolerant power supplies. They are installed in the pit on racks on both sides of the muon stations M2-M5 and in the radiation safe area under the RICH. Eight LV power supplies ${ }^{30}$ are used for the CARDIAC boards and ten for the off-detector electronics boards.

The HV cabling for the wire chambers is designed to supply independently all the gaps, i.e. potentially about 5000 channels. Two distinct power supply systems are used. The first system, developed by PNPI and the University of Florida for CMS, is based on 36-channel modules and powers the R4 and R3 chambers in M2-M5. Some of the gaps of R4 chambers are connected in parallel in groups of four via patch panels. In this way the number of independent HV channels is reduced from 3840 to 1920 . The gaps connected in parallel always belong to different chambers to minimize the loss of efficiency in case, e.g., one gap should become shorted. The second system is a commercial one based on 32-channel modules. ${ }^{31}$ It powers the chambers more exposed to

\footnotetext{
${ }^{29}$ ACTEL flash ProASICPLUS FPGA (APA450PQG208).

${ }^{30}$ MARATON low voltage power supply system for hazardous hostile environment; W-IE-NE-R, Plein \& Baus GmbH, Burscheid, Germany.

${ }^{31}$ CAEN Easy 3000 and A3535P.
} 
radiation and drawing larger currents, and for this reason all the 1152 channels can be individually controlled and monitored.

Both HV systems have been tested for radiation resistance (hadrons, total dose and neutrons) and are apt to satisfy $\mathrm{LHCb}$ requirements for 10 -year operation with a 10 -fold safety factor.

The GEM detectors require several voltages to operate. To achieve maximum flexibility and safety a customized system [210] was designed, which allows independent settings for all of the GEM electrodes for a total of 168 channels. This system is entirely installed in the counting house.

\subsubsection{Experiment control system}

The control of the muon detectors is carried out with a distributed system where the processing capacity is shared among the system nodes. CANBus was chosen because of its features of multimaster protocol with real-time capability, error correction, long distance communication and low noise. CAN nodes based on ELMBs are present in the ODE, SB and PDM boards. The implementation of calibration and test procedures in the ELMB firmware, as is the case for the SBs, maximizes the parallelism of the system and minimizes the traffic on the CANBus which is only used to communicate the results of the procedures.

All the physical channels which cannot be accessed via DAQ can be monitored via ECS while running the experiment to measure noise level and detecting dead channels. More complex procedures such as noise measurements at several thresholds to detect faulty FE channels will be performed offline, The pulse system (PDM) will allow for checks of the time alignment of the full detector.

The ECS software (see section 8.4) was developed in PVSS following the general experiment guidelines and exploiting the tools provided by the LHCb PVSS framework. The muon ECS is partitioned in two independent left-right halves and is organized in hierarchycal topological structures. Each device of the muon system, like SBs, ODE Boards, HV and LV power supplies, is seen as a Finite State Machine with well defined states and interactions with other machines.

Ten PCs running PVSS programs are used to manage all the CARDIAC, ODE and SB boards. Six are used to control the HV and LV power supplies and one to monitor the environment pressure and temperature. The entire hierarchy is controlled by two PCs, one for the left and one for the right side of the muon system.

\subsubsection{Gas system}

The gas system has been designed in collaboration with the CERN Gas Group. Due to the rather large gas volume of the MWPCs $\left(8.3 \mathrm{~m}^{3}\right)$, a recirculation system is used with a planned regeneration rate of about $90 \%$. A pump in the return line allows the gas to be compressed before entering the gas building at the surface, where the mixing and purifying units are located. The purifier in the recirculation circuit contains two cleaning agents: a molecular sieve to keep the water contamination below the level of $100 \mathrm{ppm}$, and activated copper as a reducing agent for oxygen impurities, which should be kept below the $50 \mathrm{ppm}$ level. A humidity and an oxygen meter allow for measurement of the impurity concentration before or after the purifier. Due to the small gas volume of only 10 litres, a vented system is used for the GEM chambers. 

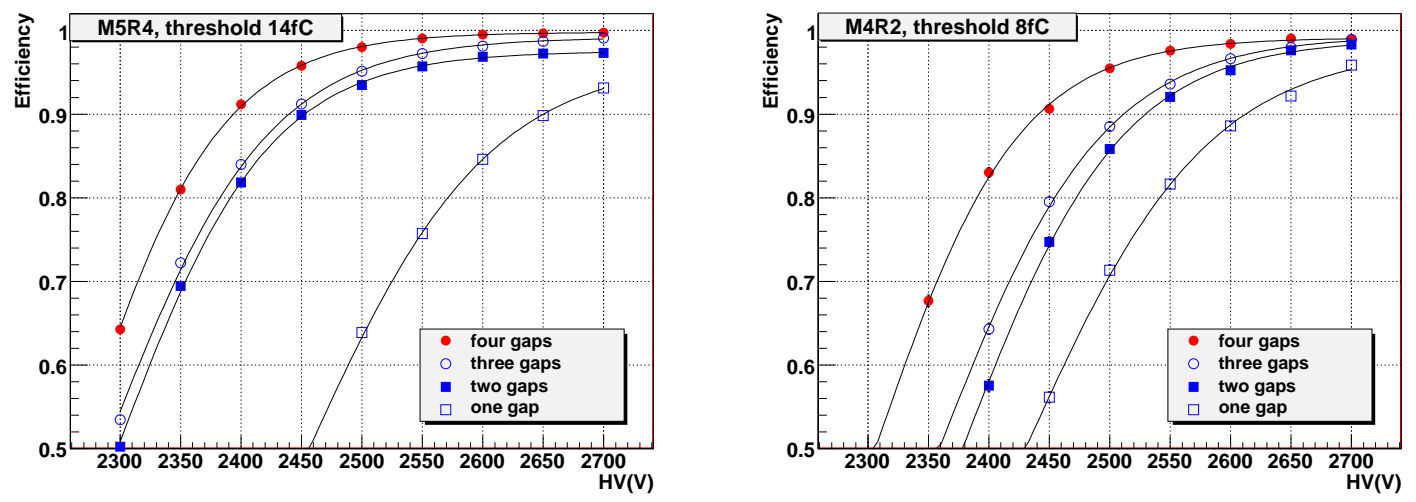

Figure 6.64: Plateau curves showing the efficiency in a $20 \mathrm{~ns}$ window for an anode-readout chamber (left) and a cathode-readout chamber (right). The four curves refer to different number of gaps being operated (see text). The threshold values in $\mathrm{fC}$ are also indicated, a lower value being used for cathode readout.

The flow rate varies across the detector surface from one volume exchange per hour, for the regions exposed to the largest irradiation (inner regions of station M1), to two volume exchanges per day for the outer regions of stations M4 and M5. Six distribution circuits are used: two for M1, two for M2-M3 and two for M4-M5. They are equipped with gas flow meters for the inputs and outputs of each supply line.

While the 12 GEM chambers have individual supply lines, only 180 supply lines are used for the 1368 MWPCs. To maintain still a parallel gas supply for each chamber, which is required to ensure an optimal performance of each individual chamber, several distributors serving 5-8 chambers have been installed on the support walls. In order to equalize the gas flow through the chambers connected to each distributor, capillaries with an impedance of about 1 mbar are introduced at the input of each chamber.

\subsubsection{Performance}

\section{MWPC}

Extensive tests were performed on prototypes and final MWPCs both in test beams and with cosmic rays at the production sites to measure the chamber performance. All measurements were conservatively done using a $20 \mathrm{~ns}$ time window.

Whereas MWPCs are expected to operate normally with four gaps in OR (two in case of M1 chambers), it is possible that a chamber will be operated with fewer gaps. Figure 6.64 shows the efficiency as a function of the applied voltage for the largest chamber with anode readout (M5R4) and for one chamber with cathode readout (M4R2). The curves are given for the standard fourgap OR configuration, but also for three-gap configuration (one double-gap fully working and the second with one gap shut off), for two gaps read by the same front-end and finally for the singlegap case. The curves show clearly the large improvement obtained by adding gaps, but also that, 

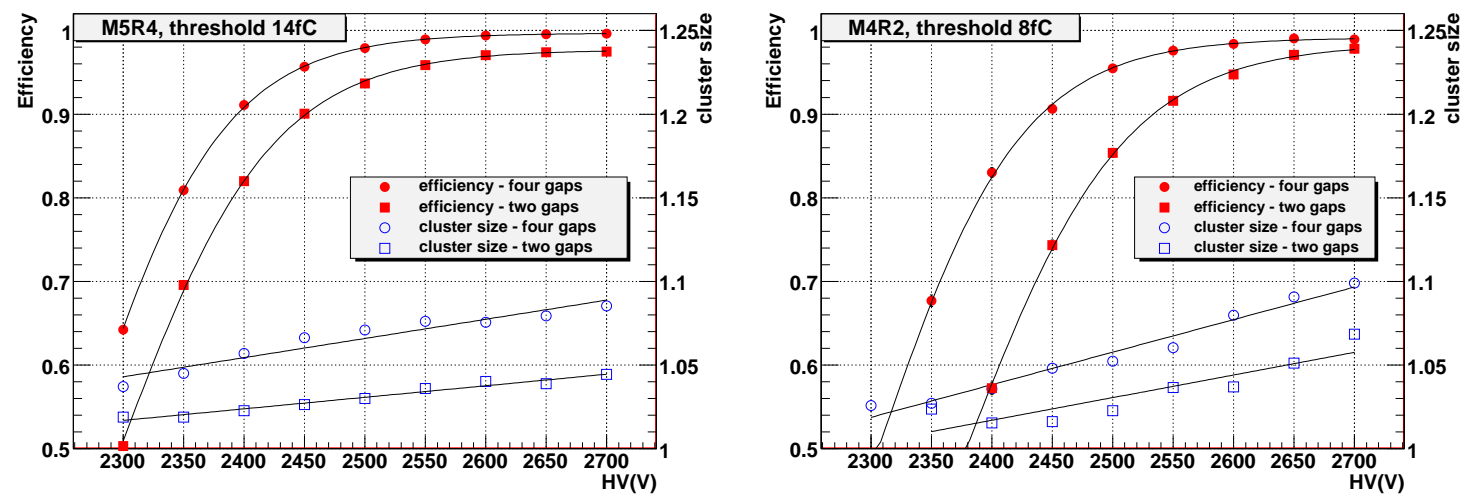

Figure 6.65: Efficiency and cluster size in a $20 \mathrm{~ns}$ window for the four-gap and double-gap configurations vs. HV. Anode readout-type chamber (left) and cathode readout-type chamber (right).

in case one gap should be shut off during the data taking, the redundancy built into the chambers will make the loss in performance negligible.

Another important parameter is the cluster size, i.e. the average number of pads fired by a crossing particle. The cluster size affects directly the space resolution and the $p_{\mathrm{T}}$ measurement in the Level-0 trigger since the yes-no readout does not allow an interpolation between adjacent pads. A non-negligible contribution to the cluster size comes simply from the geometry given the fact that inclined tracks can cross two adjacent pads belonging to different gaps. Another contribution comes from the chamber itself: inductive and capacitive cross-talk in the chamber and in the electronics. The design criteria requires that the intrinsic cluster size should be less than 1.2. This is well satisfied as can be seen in figure 6.65 which shows the cluster size distribution for the M5R4 and M2R4 chambers measured with perpendicular tracks [211]. Cosmic rays have also been used to check the gas gain uniformity on final chambers [212].

The rate capability of the MWPCs was studied at the CERN GIF [213] test beam. The detection efficiency of the chamber exposed to the intense gamma flux was measured with a $100 \mathrm{GeV}$ muon beam. The chamber performance was studied for several detector gap configurations and different values of the background rate from the source.

No effect was detected at the maximum gamma rate allowed at the GIF where a current density of $28 \mathrm{nA} / \mathrm{cm}^{2}$ was measured at a high voltage of $2.75 \mathrm{kV}$. This value is about the one expected in the chamber with the highest occupancy in the nominal conditions of running. This is in agreement with the results of the simulations [179] which show that space charge effects due to accumulation of ions are expected only at much higher currents.

\section{GEM chambers}

Triple-GEM detectors with the final FE electronics were tested both in a dedicated $40 \mathrm{MHz}$ test beam and with cosmics in the lab.

Efficiency and cluster size in a $20 \mathrm{~ns}$ window, as a function of gain, are shown in figure 6.66 for a chamber composed of two triple-GEM detectors. Thanks to the longer shaping time of the CARIOCAGEM with respect to the standard CARIOCA chip the beginning of the efficiency plateau 

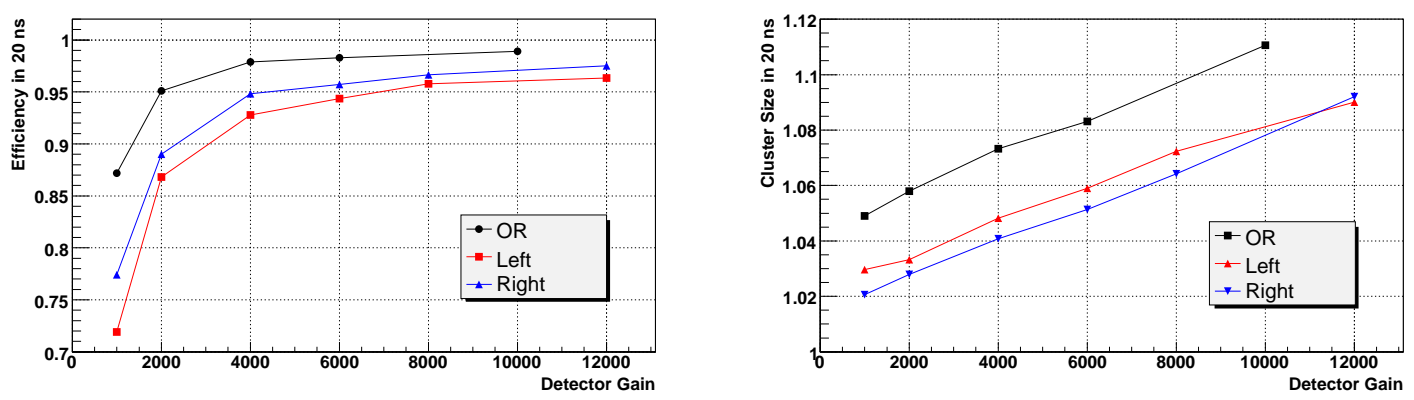

Figure 6.66: Efficiency and cluster size in a $20 \mathrm{~ns}$ window vs. gain for a chamber composed of two triple-GEM detectors. The curves show the data for the single detectors and for the OR of the two.

has moved from a detector gain of 6000 to 4000 . This allows to operate the chambers with an integrated charge decreased by $30 \%$ and in safer conditions with respect to discharges. 


\section{Chapter 7}

\section{Trigger}

The LHCb experiment plans to operate at an average luminosity of $2 \times 10^{32} \mathrm{~cm}^{-2} \mathrm{~s}^{-1}$, much lower than the maximum design luminosity of the LHC, reducing the radiation damage to the detectors and electronics. Futhermore, the number of interactions per bunch crossing is dominated by single interactions, which facilitates the triggering and reconstruction by assuring low channel occupancy. Due to the LHC bunch structure and low luminosity, the crossing frequency with interactions visible $^{1}$ by the spectrometer is about $10 \mathrm{MHz}$, which has to be reduced by the trigger to about $2 \mathrm{kHz}$, at which rate the events are written to storage for further offline analysis. This reduction is achieved in two trigger levels [214] as shown in figure 7.1: Level-0 (L0) and the High Level Trigger (HLT). The L0 trigger is implemented using custom made electronics, operating synchronously with the $40 \mathrm{MHz}$ bunch crossing frequency, while the HLT is executed asynchronously on a processor farm, using commercially available equipment. At a luminosity of $2 \times 10^{32} \mathrm{~cm}^{-2} \mathrm{~s}^{-1}$ the bunch crossings with visible pp interactions are expected to contain a rate of about $100 \mathrm{kHz}$ of b $\bar{b}$-pairs. However, only about $15 \%$ of these events will include at least one B meson with all its decay products contained in the spectrometer acceptance. Furthermore the branching ratios of interesting B meson decays used to study for instance CP violation are typically less than $10^{-3}$. The offline analysis uses event selections based on the masses of the B mesons, their lifetimes and other stringent cuts to enhance the signal over background. For the best overall performance the trigger was therefore optimised to achieve the highest efficiency for the events selected in the offline analysis, while rejecting uninteresting background events as strongly as possible.

The purpose of the L0 trigger is to reduce the LHC beam crossing rate of $40 \mathrm{MHz}$ to the rate of $1 \mathrm{MHz}$ with which the entire detector can be read out. Due to their large mass, B mesons decays often produce particles with large transverse momentum $\left(p_{\mathrm{T}}\right)$ and energy $\left(E_{\mathrm{T}}\right)$ respectively. The Level-0 trigger attempts to reconstruct:

- the highest $E_{\mathrm{T}}$ hadron, electron and photon clusters in the calorimeters,

- the two highest $p_{\mathrm{T}}$ muons in the muon chambers.

In addition, a pile-up system in the VELO estimates the number of primary pp interactions in each bunch crossing. The calorimeters calculate the total observed energy and an estimate for the num-

\footnotetext{
${ }^{1}$ An interaction is defined to be visible if it produces at least two charged particles with sufficient hits in the VELO and $\mathrm{T} 1-\mathrm{T} 3$ to allow them to be reconstructible.
} 


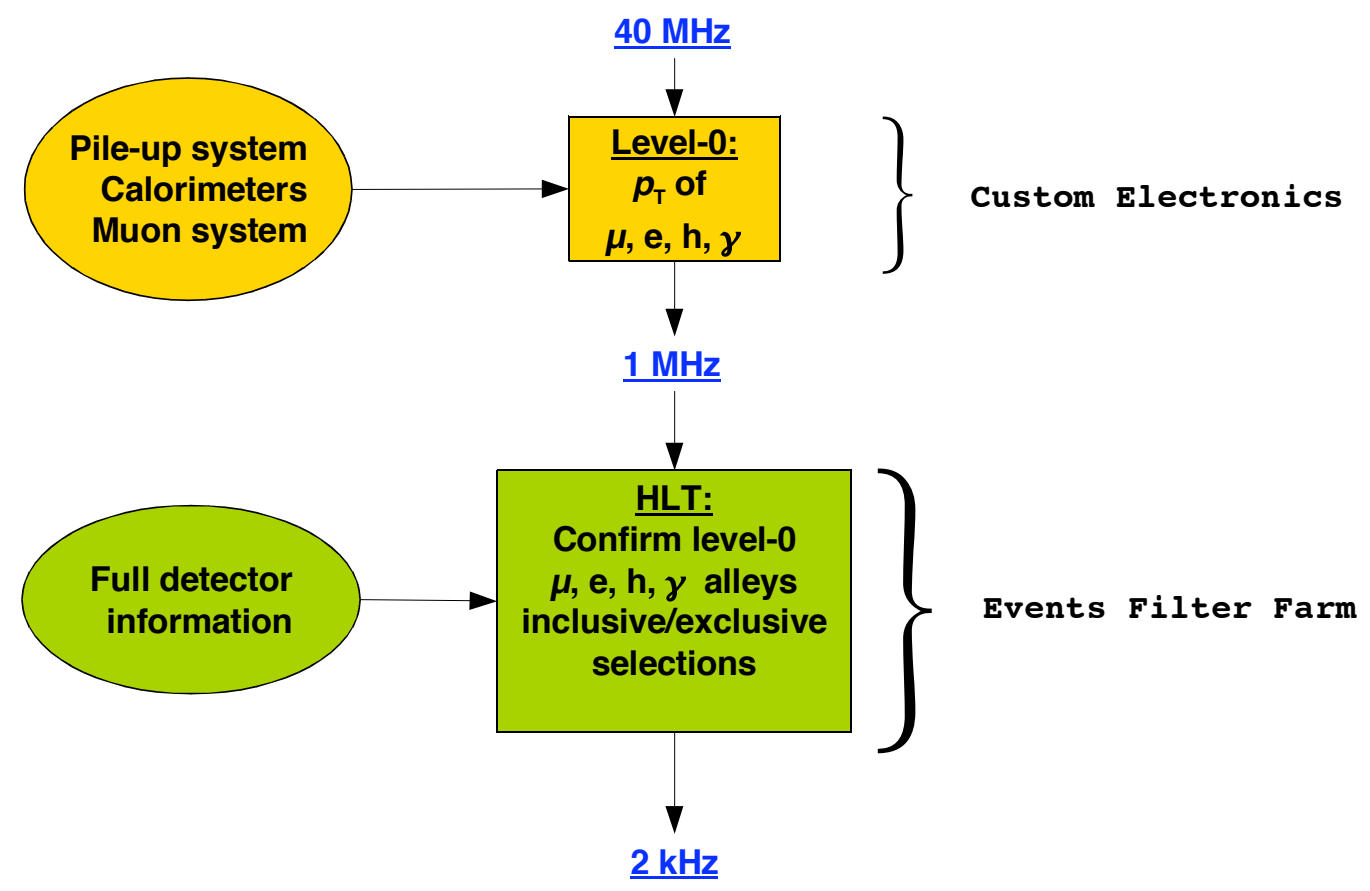

Figure 7.1: Scheme of the LHCb trigger.

ber of tracks, based on the number of hits in the SPD. With the help of these global quantities events may be rejected, which would otherwise be triggered due to large combinatorics, and would occupy a disproportionate fraction of the data-flow bandwidth or available processing power in the HLT.

A Level-0 Decision Unit (DU) collects all the information and derives the final Level-0 trigger decision for each bunch crossing. It allows for overlapping of several trigger conditions and for prescaling.

The L0 trigger system is fully synchronous with the $40 \mathrm{MHz}$ bunch crossing signal of the LHC. The latencies are fixed and depend neither on the occupancy nor on the bunch crossing history. All Level-0 electronics is implemented in fully custom-designed boards which make use of parallelism and pipelining to do the necessary calculations with sufficient speed.

In order to be able to reduce the event rate from $1 \mathrm{MHz}$ down to $2 \mathrm{kHz}$, the HLT makes use of the full event data. The generic HLT algorithms refine candidates found by the Level-0 trigger and divide them into independent alleys (see section 7.2). The alleys to be followed are selected from the Level-0 decision. The alley selections are based on the principle of confirming a previous trigger stage by requiring the candidate tracks to be reconstructed in the VELO and/or the T-stations. Requiring candidate tracks with a combination of high $p_{\mathrm{T}}$ and/or large impact parameter reduces the rate to about $30 \mathrm{kHz}$. At this rate interesting final states are selected using inclusive and exclusive criteria.

Generally speaking, selection cuts are relaxed compared to the offline analysis, in order to be able to study the sensitivity of the selections and to profit from refinements due to improved calibration constants. A large fraction of the output bandwidth is devoted to calibration and monitoring. In order to monitor trigger efficiencies and systematic uncertainties both trigger levels can be emulated fully on stored data. 


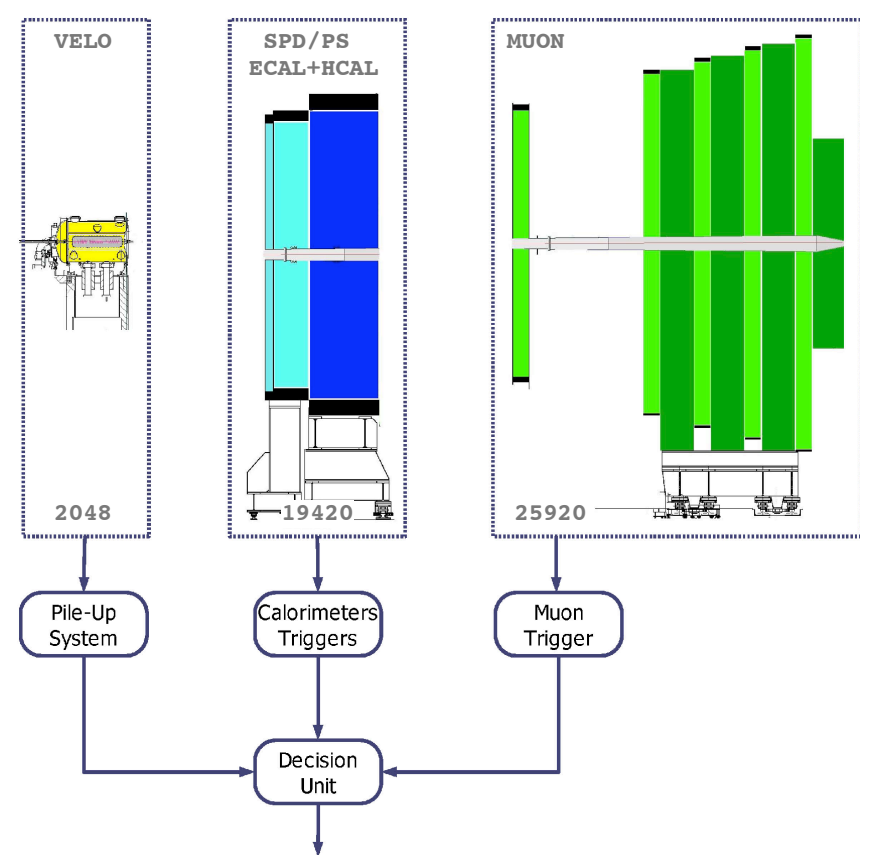

Figure 7.2: Overview of the Level-0 trigger. Every $25 \mathrm{~ns}$ the pile-up system receives 2048 channels from the pile-up detector, the Level-0 calorimeters 19420 channels from the scintillating pad detector, preshower, electromagnetic and hadronic calorimeters while the Level-0 muon handles 25920 logical channels from the muon detector.

\subsection{Level 0 trigger}

\subsubsection{Overview}

As shown in figure 7.2, the Level-0 trigger is subdivided into three components: the pile-up system, the Level-0 calorimeter trigger and the Level-0 muon trigger. Each component is connected to one detector and to the Level-0 DU which collects all information calculated by the trigger systems to evaluate the final decision.

The pile-up system aims at distinguishing between crossings with single and multiple visible interactions. It uses four silicon sensors of the same type as those used in the VELO to measure the radial position of tracks. The pile-up system provides the position of the primary vertices candidates along the beam-line and a measure of the total backward charged track multiplicity.

The Calorimeter Trigger system looks for high $E_{\mathrm{T}}$ particles: electrons, $\gamma^{\prime}$ s, $\pi^{0}$ 's or hadrons. It forms clusters by adding the $E_{\mathrm{T}}$ of $2 \times 2$ cells and selecting the clusters with the largest $E_{\mathrm{T}}$. Clusters are identified as electron, $\gamma$ or hadron based on the information from the SPD, PS, ECAL and HCAL Calorimeter. The $E_{\mathrm{T}}$ of all HCAL cells is summed to reject crossings without visible interactions and to reject triggers on muon from the halo. The total number of SPD cells with a hit are counted to provide a measure of the charged track multiplicity in the crossing.

The muon chambers allow stand-alone muon reconstruction with a $p_{\mathrm{T}}$ resolution of $\sim 20 \%$. Track finding is performed by processing elements which combine the strip and pad data from the five muon stations to form towers pointing towards the interaction region. The Level-0 muon trigger selects the two muons with the highest $p_{\mathrm{T}}$ for each quadrant of the muon detector. 


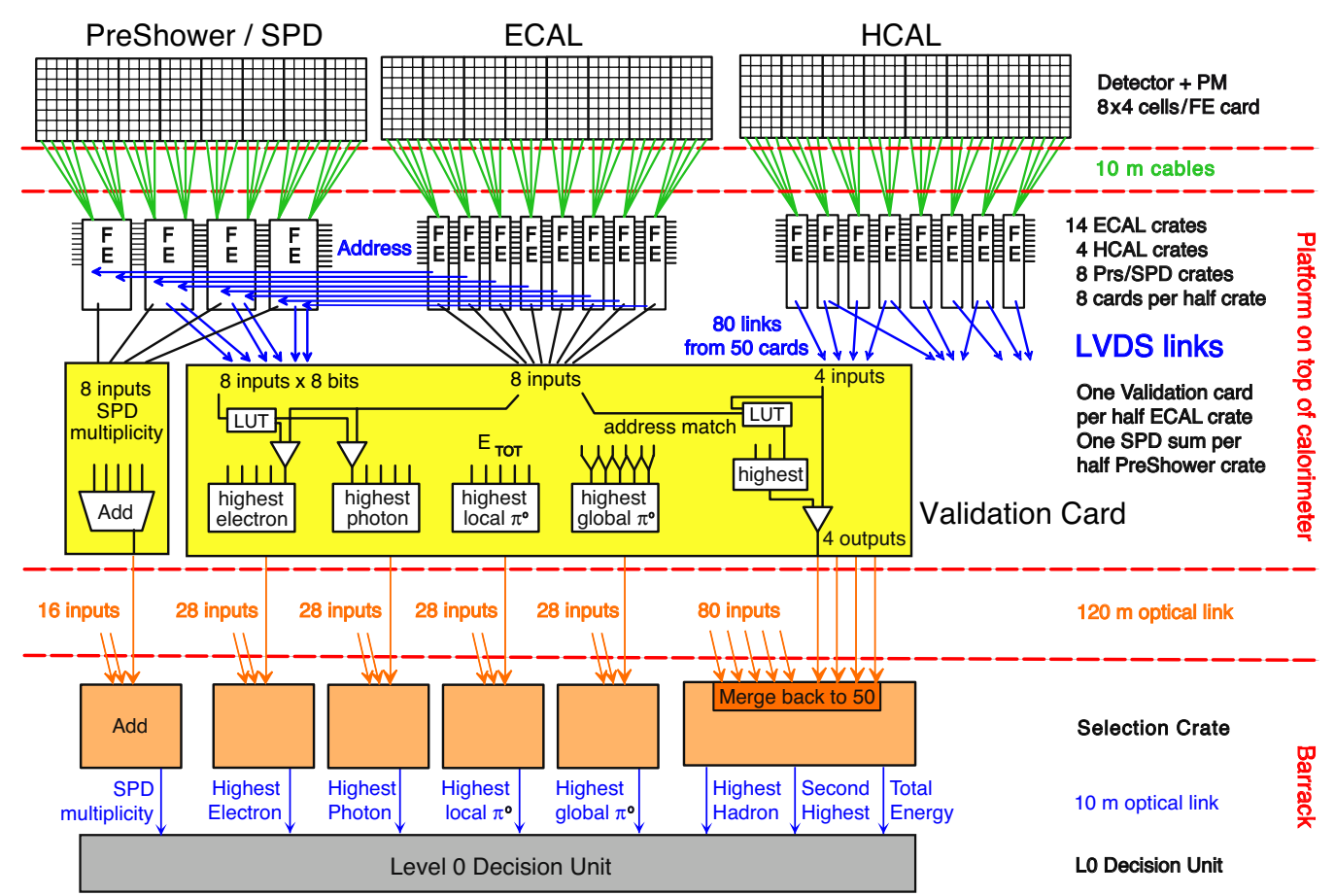

Figure 7.3: Overview of the Level-0 calorimeter trigger architecture.

The Level-0 DU collects all information from Level-0 components to form the Level-0 trigger. It is able to perform simple logic to combine all signatures into one decision per crossing. This decision is passed to the Readout Supervisor (see section 8.3) which transmits it to the front-end electronics.

The latency of Level-0, i.e. the time elapsed between a pp interaction and the arrival of the Level-0 trigger decision at the front-end electronics, is fixed to $4 \mu \mathrm{s}$. This time which includes the time-of-flight of the particles, cable delays and all delays in the front-end electronics, leaves $2 \mu \mathrm{s}$ for the processing of the data in the Level-0 trigger to derive a decision.

\subsubsection{Architecture}

\section{Calorimeter trigger}

A zone of 2 by 2 cells is used, since it is large enough to contain most of the energy, and small enough to avoid overlap of various particles. Ultimately, only the particle with the highest $E_{\mathrm{T}}$ enters into the trigger decision. Therefore, to minimize the number of candidates to be processed, only the highest $E_{\mathrm{T}}$ candidate is kept at this stage.

These candidates are provided by a three step selection system as shown in figure 7.3:

- a first selection of high $E_{\mathrm{T}}$ deposits is performed on the Front-End card, which is the same for ECAL and HCAL. Each card handles 32 cells, and the highest $E_{\mathrm{T}}$ sum over the 32 sums of $2 \times 2$ cells is selected. To compute these 32 sums, access to cells in other cards is an important issue. 
- the Validation Card merges the ECAL with the PS and SPD information prepared by the preshower front-end card. It identifies the type of electromagnetic candidate, electron, $\gamma$ or $\pi^{0}$. Only the highest $E_{\mathrm{T}}$ candidate per type is selected and sent to the next stage. The same card also adds the energy deposited in ECAL to the corresponding hadron candidates. Similar cards in the PreShower crates compute the SPD multiplicity.

- the Selection Crate selects the candidate with the highest $E_{\mathrm{T}}$ for each type, and also produces a measure of the total $E_{\mathrm{T}}$ in HCAL and the total SPD multiplicity.

The first two steps are performed on the calorimeter platform, at a location where the radiation dose is expected to be below $50 \mathrm{~Gy}$ over the whole lifetime of the experiment, and where single event upsets are expected to occur. Each component has been tested for radiation tolerance and robustness against single event upsets. Anti-fuse FPGAs are used, as well as triple-voting techniques.

The trigger interface is housed in one anti-fuse FPGA from ACTEL for ECAL/HCAL frontend cards and in one flash EEPROM based FPGA for PS/SPD front-end boards. There is a large data flow between these components at a frequency of $40 \mathrm{MHZ}$, through a dedicated backplane, where interconnections are realized by point-to-point links running a multiplexed LVDS signals at $280 \mathrm{MHz}$. The same backplane is used for PreShower, ECAL and HCAL crates.

The validation card is a 9U board with 16 layers. Clusters, PS and SPD hit maps arrive through the backplane via 20 LVDS links running at $280 \mathrm{MHz}$. The cluster identification is performed by two ProAsic FPGAs from ACTEL. Electron, $\gamma$, hadron and $\pi^{0}$ candidates are transmitted to the selection crate via an 8-channel optical mezzanine which serializes data at $1.6 \mathrm{Gbps}$ and drives a ribbon of 12 fibres. The control of the validation and calorimeter front-end cards are performed by a SPECS interface.

The selection crate is located in the counting house in a radiation free environment. It is a modular system containing eight 16-layer 9U VME selection boards. The design of the selection boards is unique and adapted to perform both the electromagnetic and the hadron clusters selection. The electromagnetic cluster selection is performed on one board for each cluster type (electron, $\gamma$, $\pi^{0}$ ) while the hadron selection requires three boards. The results of the two first boards are transmitted to the third one where the final selection is performed. Finally, one board is used to sum the SPD multiplicity. Inputs arrive via 28 optical links grouped into three ribbons of 12 fibres. High-speed serial signals are deserialized by 28 TLK2501 chips. ${ }^{2}$ The selection of the highest $E_{\mathrm{T}}$ candidate of each type is performed by six FPGAs from the Xilink Virtex II family. The selected candidates are sent to the Level-0 DU via a mezzanine with 1-channel high speed optical link. Inputs and outputs of the Selection Boards are sent to the data acquisition system via two high speed optical links connected to the TELL1 board. The Selection Boards are controlled by a credit card PC.

The types and total numbers of boards for the Level-0 Calorimeters Trigger are summarized in table 7.1. 
Table 7.1: Boards of the Level-0 calorimeters trigger.

\begin{tabular}{|lr|}
\hline Boards & Number \\
\hline \hline ECAL/HCAL front-end & 246 \\
PS/SPD front-end & 100 \\
8-channels optical mezzanine & 80 \\
1-channels optical mezzanine & 40 \\
Validation card & 28 \\
SPD Control board & 16 \\
Selection Board & 8 \\
\hline
\end{tabular}

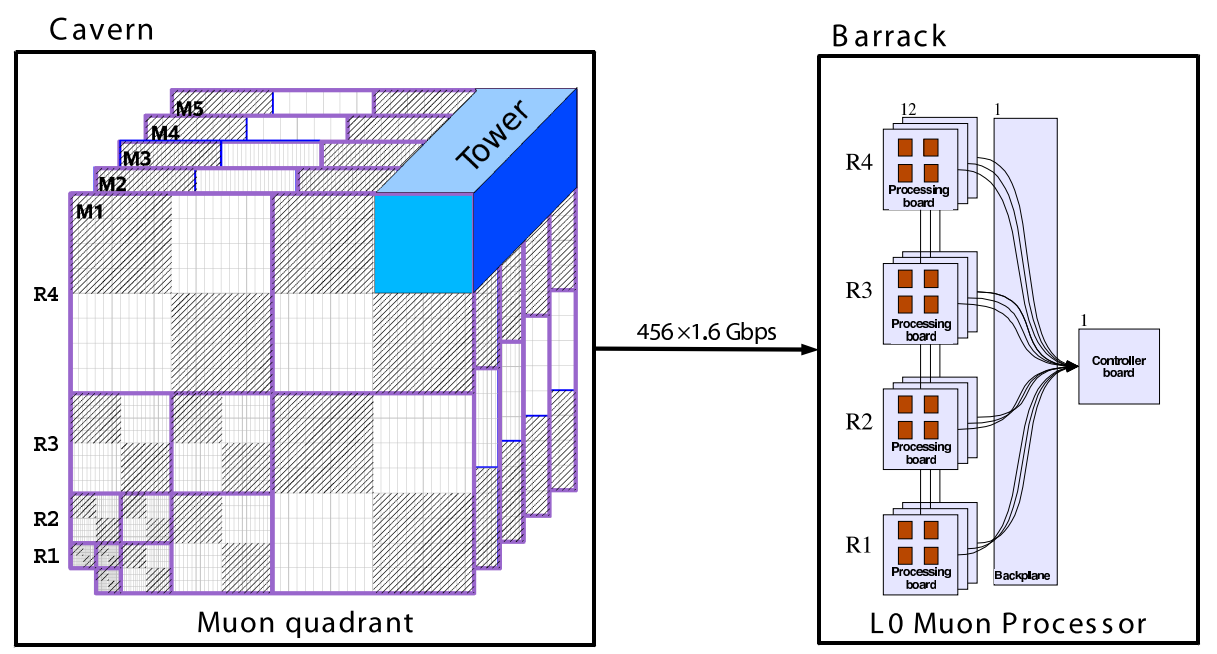

Figure 7.4: Overview of the Level-0 muon architecture.

\section{Muon trigger}

An overview of the Level-0 muon architecture is given in figure 7.4 and a detailed description in [215]. Each quadrant of the muon detector is connected to a Level-0 muon processor via 456 optical links grouped in 38 ribbons containing 12 optical fibres each. An optical fibre transmits serialized data at $1.6 \mathrm{Gbps}$ over a distance of approximately 100 meters. The 4 Level- 0 muon processors are located in the counting house, a place immune to radiation effects.

A L0 muon processor looks for the two muon tracks with the largest and second largest $p_{\mathrm{T}}$. The track finding is performed on the logical pads. It searches for hits defining a straight line through the five muon stations and pointing towards the interaction point. The position of a track in the first two stations allows the determination of its $p_{\mathrm{T}}$. The final algorithm is very close to the one reported in the Technical Proposal [1] and in the Muon Technical Design Report [164].

Seeds of the track finding algorithm are hits in M3. For each logical pad hit in M3, an extrapolated position is set in M2, M4 and M5 along a straight line passing through the hit and the interaction point. Hits are looked for in these stations in search windows termed Field Of Interest (FOI) which are approximately centred on the extrapolated positions. FOIs are opened along the $x$ -

\footnotetext{
${ }^{2}$ from Texas Instrument, USA.
} 
axis for all stations and along the $y$-axis only for stations M4 and M5. The size of the FOI depends on the station being considered, the level of background and the minimum-bias retention allowed. When at least one hit is found inside the FOI for each station M2, M4 and M5, a muon track is flagged and the pad hit in M2 closest to the extrapolation from M3 is selected for a subsequent use.

The track position in station M1 is determined by making a straight-line extrapolation from M3 and M2, and identifying, in the M1 FOI, the pad hit closest to the extrapolation point.

Since the logical layout is projective, there is a one-to-one mapping from pads in M3 to pads in M2, M4 and M5. There is also a one-to one mapping from pairs of pads in M2 and M3 to pads in M1. This allows the track finding algorithm to be implemented using only logical operations.

To simplify the processing and to hide the complex layout of the stations, the muon detector is subdivided into 192 towers (48 per quadrant) pointing towards the interaction point. All towers have the same layout with 288 logical pads ${ }^{3}$ each. Therefore, the same algorithm can be executed in each tower. Each tower is connected to a processing element, the basic component of the Level-0 Muon processor.

To collect data coming from a tower spread over five stations and to send them to the processing element, a patch panel close to the muon processor is used.

Processing elements have to exchange a large number ${ }^{4}$ of logical channels with each other to avoid inefficiencies on borders of towers. The topology of the data exchange depends strongly on the location of the tower.

A processing element runs 96 tracking algorithms in parallel, one per M3 seed, on logical channels from a tower. It is implemented in a FPGA named Processing Unit (PU). A processing board contains four PUs and an additional FPGA to select the two muons with the highest transverse momentum within the board. A Level-0 Muon processor consists of a crate housing 12 Processing Boards, a custom backplane and a controller board. The custom backplane is mandatory to exchange logical channels between PUs. The controller board collects candidates found by the 12 Processing Boards and selects the two with the highest $p_{\mathrm{T}}$. It also distributes signals coming from the TTC.

The Level-0 Muon implementation relies on the massive use of multigigabit serial links deserialized inside FPGAs. Processors are interfaced to the outside world via optical links while processing elements are interconnected with high speed copper serial links.

The Processing Board contains five FPGAs from the Altera Stratix GX family and 92 high speed serial links with serialiazers and deserializers embedded in FPGAs. The board sends data to the data acquisition system via two high speed optical links. The processing board is remotely controlled via Ethernet by a credit card PC running Linux. The size of the printed circuit is $366.7 \times$ $220 \mathrm{~mm}$ and is composed of 18 layers and a total of 1512 components. The power consumption is less than $60 \mathrm{~W}$.

The Controller Board contains two FPGAs from the Stratix GX family. The board shares many common functionalities with the Processing Board: the same credit card PC, the same mechanism to send information to the data acquisition system. The printed circuit measues

\footnotetext{
348 pads from M1, $2 \times 96$ pads from M2 and M3, $2 \times 24$ pads from M4 and M5.

${ }^{4}$ A processing element handles 288 logical pads. It sends a maximum of 224 and receives a maximum of 214 logical channels from neighbouring elements.
} 
Table 7.2: Boards of the Level-0 muon trigger.

\begin{tabular}{|lr|}
\hline Boards & Number \\
\hline \hline Processing Board & 48 \\
Controller Board & 4 \\
Backplane & 4 \\
\hline
\end{tabular}

$366.7 \times 220 \mathrm{~mm}$ and is composed of 14 layers with 948 mounted components. The power consumption is less than $50 \mathrm{~W}$.

The backplane contains 15 slots: 12 for the Processing Boards, one for the Controller Board and two for test. It distributes power supplies, signals coming from the TTC, and assures the connectivity between the processing elements via 288 single-ended links $(40 \mathrm{MHz})$ and 110 differential high speed serial links $(1.6 \mathrm{Gbps})$. The size of the 18-layer printed circuit board is $395,4 \times 426,72 \mathrm{~mm}$.

The types and total numbers of boards for the Level-0 Muon Trigger are summarized in table 7.2.

\section{Pile-Up system}

The pile-up system consists of two planes $(A$ and $B$ ) perpendicular to the beam-line and located upstream of the VELO (see figure 5.1). Each $300 \mu \mathrm{m}$ thick silicon plane consists of two overlapping VELO R-sensors which have strips at constant radii, and each strip covers $45^{\circ}$. In both planes the radii of track hits, $r_{a}$ and $r_{b}$, are measured. The hits belonging to tracks from the same origin have the simple relation $k=r_{b} / r_{a}$, giving:

$$
z_{v}=\frac{k z_{a}-z_{b}}{k-1}
$$

where $z_{b}, z_{a}$ are the detector positions and $z_{v}$ is the position of the track origin on the beam axis, i.e. the vertex. The equation is exact for tracks originating from the beam-line. All hits in the same octant of both planes are combined according to equation 7.1 and the resulting values of $z_{v}$ are entered into an appropriately binned histogram, in which a peak search is performed, as shown in figure 7.5. The resolution of $z_{v}$ is limited to around $3 \mathrm{~mm}$ by multiple scattering and the hit resolution of the radial measurements. All hits contributing to the highest peak in this histogram are masked, after which a second peak is searched for. The height of this second peak is a measure of the number of tracks coming from a second vertex. A cut is applied on this number to detect multiple interactions. If multiple interactions are found, the crossing is vetoed.

The architecture of the pile-up system is shown in figure 7.6. It uses the signals of the integrated comparators of the Beetle chips located on the four hybrids. The outputs of neighbouring comparators are OR-ed in groups of four, resulting in 256 LVDS links running at $80 \mathrm{Mbit} / \mathrm{s}$ per hybrid, which send the Level-0 signals to eight Optical Transmission Boards. Two Optical Transmission Boards cover one quadrant. They time align and multiplex input hit maps to four Vertex Processing Boards. Hit maps of one bunch crossing are sent to one of the four Vertex Processing Board (VEPROB) in four consecutive clock cycles, while hit maps of the following bunch crossing 

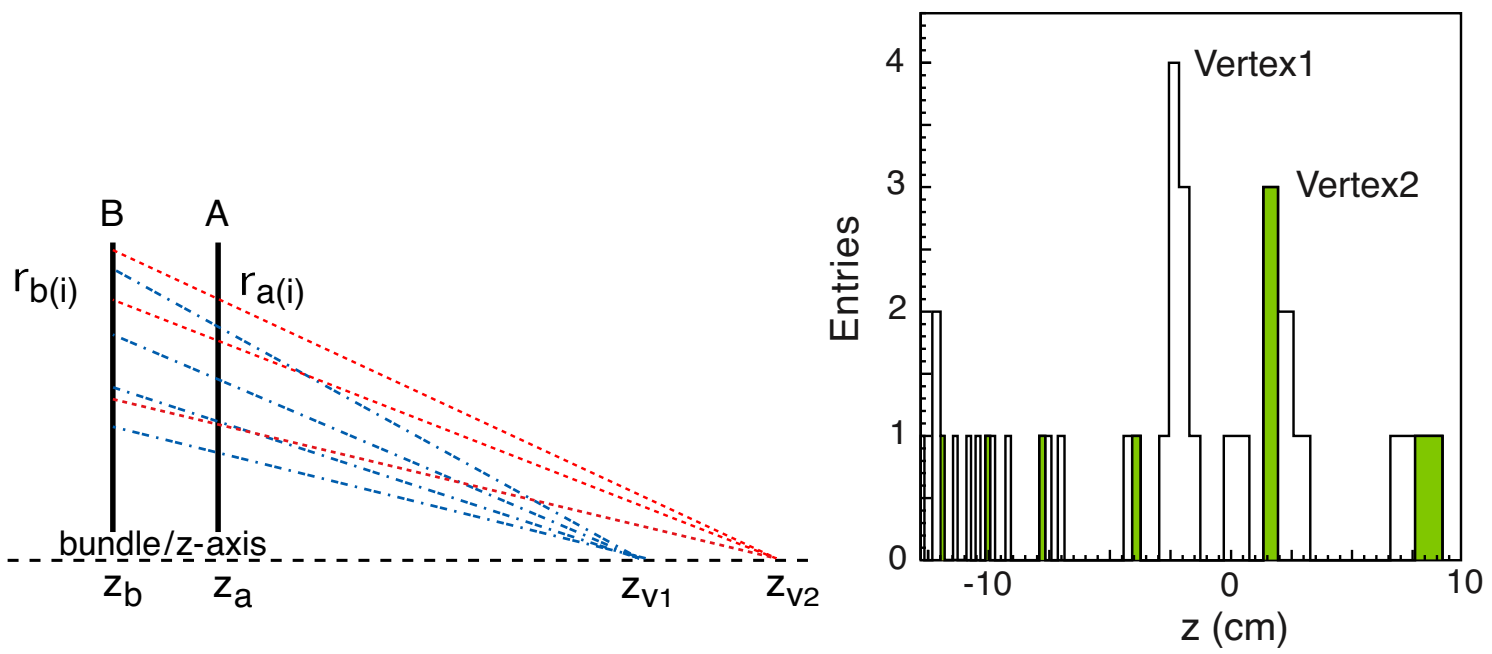

Figure 7.5: The basic principle of detecting vertices in an event. The hits of plane A and B are combined in a coincidence matrix. All combinations are projected onto a $z_{v}$-histogram. The peaks indicated correspond to the two interaction vertices in this particular MonteCarlo event. After the first vertex finding iteration, the hits corresponding to the two highest bins are masked, resulting in the hatched histogram.

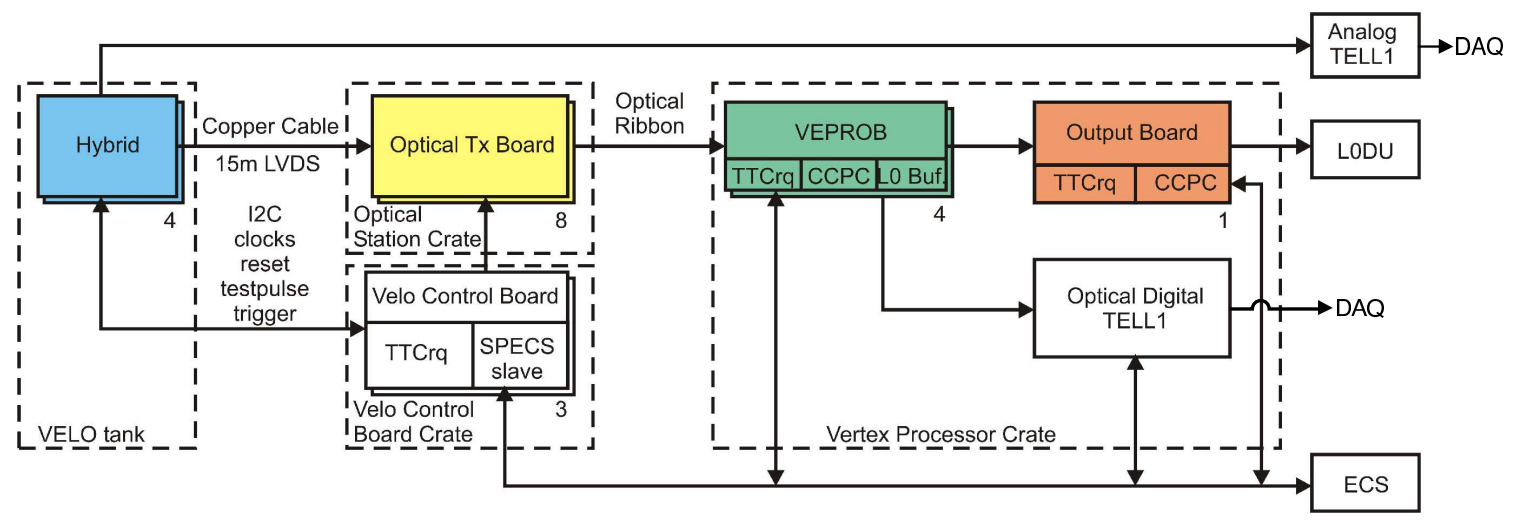

Figure 7.6: Overview of the Level-0 pile-up Architecture.

are sent to the second VEPROB in four consecutive clock cycles. Bunch-crossings are distributed over the four Vertex Processing Boards in a round-robin fashion. The Optical transmision board is a 9 U board controlled by a SPEC interface via the VELO control board.

Vertex Processing boards are $9 \mathrm{U}$ boards located in the radiation-free electronics barracks. They are connected to the Optical Transmission boards via 24 high speed optical links. The vertex processing board is the key component of the pile-up system. It houses a large FPGA from Xilinx Virtex II family which runs the pile-up algorithm. A board handles one of four events and sends its trigger decision to the output board via a high speed copper link (1.6 Gbps). The VEPROB is controlled by a credit card PC and sends the inputs and outputs of the vertex finding algorithm to the DAQ system via two high speed optical links.

The output board is a simple $9 \mathrm{U}$ board multiplexing the inputs from the vertex processing 
Table 7.3: Boards of the pile-up system.

\begin{tabular}{|lr|}
\hline Boards & Number \\
\hline \hline Hybrids & 4 \\
Optical Transmition Board & 8 \\
Vertex Processing Board & 4 \\
Output Board & 1 \\
\hline
\end{tabular}

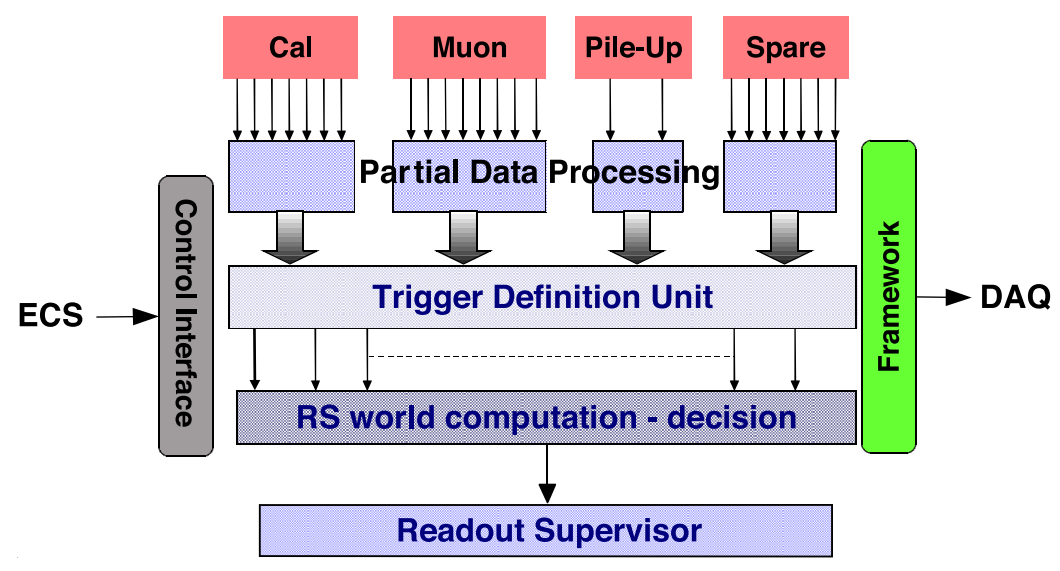

Figure 7.7: Level-0 DU architecture.

board and sends the number of primary pp interactions for each bunch crossing to the Level-0 DU. In addition, the output boards make histograms of trigger decisions made by the pile-up system. These histograms are accessible via the ECS interface.

The types total numbers of boards for the pile-up system are summarized in table 7.3.

\section{Decision Unit}

The Level-0 DU receives information from the calorimeter, muon and pile-up sub-triggers at $40 \mathrm{MHz}$, which arrive at different fixed times. The computation of the decision can start with a sub-set of information coming from a Level-0 sub-trigger, after which the sub-trigger information is time aligned. An algorithm is executed to determine the trigger decision. The decision is sent to the Readout Supervisor, which makes the ultimate decision about whether to accept an event or not. The Readout Supervisor is able to generate and time-in all types of self-triggers (random triggers, calibration, etc.) and to control the trigger rate by taking into account the status of the different components in order to prevent buffer overflows and to enable/disable the triggers at appropriate times during resets.

The architecture of the Level-0 DU is shown in figure 7.7. For each data source, a Partial Data Processing system performs a specific part of the algorithm and the synchronisation between the various data sources. Then a trigger definition unit combines the information from the above systems to form a set of trigger conditions based on multi-source information.

The trigger conditions are logically OR-ed to obtain the Level-0 decision after they have been individually downscaled if necessary. 


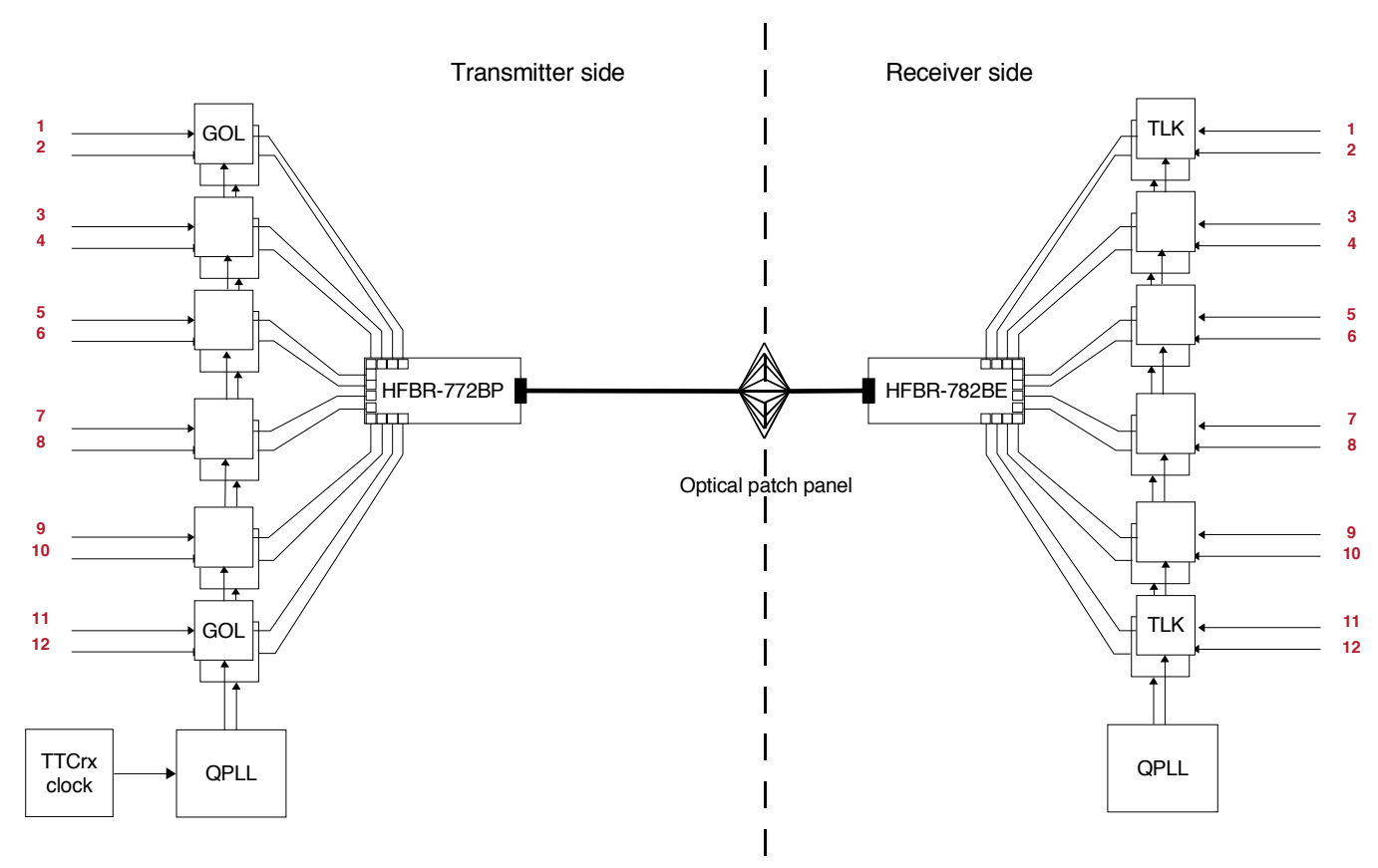

Figure 7.8: Overview of ribbon optical link.

The Level-0 DU is based on the TELL1 board with optical cards replaced by a single mezzanine in which Level-0 DU hardware is implemented. Inputs are received on two ribbons of 12 high speed optical links. Serial signals are deserialized by a 24 TLK2501 chip. ${ }^{5}$ and sent to two large FPGAs from the Stratix Family. Electron, $\gamma, \pi^{0}$, hadron and muon candidates as well as intermediate and final decisions are sent to the DAQ via the TELL1 mother boards. This information can be used later on by the HLT to confirm the Level- 0 candidates using more refined algorithms.

\subsubsection{Technology}

The implementation of the Level-0 trigger relies on the massive use of large FPGAs, high speed serial links and common techniques which simplify debugging and commissioning.

\section{High speed links}

The transport of information from the front-end electronics to Level-0 trigger boards located in the barrack is based on three concepts:

- serialization of the detector data;

- use of optical links as transport media;

- use of high density devices.

High speed serial transmission reduces the number of signal lines required to transmit data from one point to another. It also offers a high level of integration with many advantages: high reliability

\footnotetext{
${ }^{5}$ from Texas Instrument, USA.
} 
for data transfer over 100 meters; complete electrical isolation avoids ground loops and common mode problems. In addition, the integration of several high speed optical links in a single device increases data rate while keeping a manageable component count and a reasonable cost.

Ribbon optical links integrate twelve optical transmitters (fibres, receivers) in one module. The important benefit of ribbon optical links is based on low-cost array integration of electronic and opto-electronic components. It also results a low power consumption and a high level of integration.

An overview of the ribbon optical link developed for the Level-0 trigger is shown in figure 7.8. The emitter stage relies on twelve serializer chips connected to one optical transmitter. The serializer is the GOL, a radiation hard chip designed by the CERN microelectronic group, which every $25 \mathrm{~ns}$, transforms a 32-bit word into a serial signal with a frequency of $1.6 \mathrm{GHz}$ using a $8 \mathrm{~B} / 10 \mathrm{~B}$ encoding. High frequency signals are converted into optical signals by the 12-channel optical transmitter from Agilent HFBR-772BE. The module is designed to operate multimode fibres at a nominal wavelength of $850 \mathrm{~nm}$.

Initially the LHC clock distribution was not intended to be used for optical data transmission and hence, does not fulfill the severe jitter constraints required by high speed serializers. The GOL requires a maximum jitter of 100 ps peak to peak to operate correctly whereas the LHC clock jitter is as large as 400 or 500 ps. To reduce the jitter, a radiation hard chip, the QPLL, designed by the CERN microelectronics group is used. It filters out the jitter up to an acceptable value with the help of a reference quartz crystal associated to a phase locked loop.

The emitter side is close to the detector in a place where the radiation dose is below $50 \mathrm{~Gy}$ over 10 years where single event upsets (SEU) are expected to occur. The GOL and QPLL chips are radiation hard chips immune to SEU. However, the optical transceiver is a commercial component designed to work in an environment free of radiation. An irradiation campaign took place at the Paul Scherrer Institute in December 2003. The component was found to work within its specifications up to a total dose of $150 \mathrm{~Gy}$. The cross-section for single event upsets is equal to $(4.1 \pm 0.1) \times 10^{-10} \mathrm{~cm}^{2}$ per single optical link. The expected SEU rate is 1 every 220 minutes for the Level-0 muon trigger. When this happens, a single optical link emitter is not synchronized with its receiver anymore. All emitter/receiver pairs are resynchronized automatically at the end of each LHC cycle. Therefore, the link will not transmit data during a maximum of one LHC cycle or $89 \mu \mathrm{s}$. The corresponding inefficiency is negligible.

The physical media between the front-end electronic boards and the Level-0 trigger board consist of ribbons of twelve fibres with MPO connectors on both sides $(\sim 10 \mathrm{~m}$.), MPO-MPO patch panels, long cables containing eight ribbons with MPO connectors $(\sim 80 \mathrm{~m}$.), fanout panels (MPO-MPO or MPO-SC), short ribbons of twelve fibres $(\sim 3 \mathrm{~m})$ with MPO connector on one side and a MPO or 12 SC connectors on the other side.

The receiving side is the mirror of the emitting side. Optical signals are converted into $1.6 \mathrm{Gbps}$ serial electrical signals by the 12-channel optical receiver HFBR-782BE. The twelve high-frequency signals are deserialized into 16-bit words at $80 \mathrm{MHz}$ by twelve TLK2501 chips. The receiving side is located in the counting room. Therefore standard components can be used. In the muon processing board, where the density of input signal is high, TLK2501 chips are replaced by serializers and deserializers embedded in the Stratix GX FPGA. 
The routing of the differential high speed traces between serializer/deserializer and the optical transceiver requires considerable care since the geometry of the tracks must be totally controlled to guarantee good impedance matching and to minimize electromagnetic emissions to the environment as well as sensitivity to electromagnetic perturbations from the environment.

The performance of the optical link has been measured with several setups in different ways. The bit error ratio measured with Lecroy SDA11000 Serial Data Analyser is below $10^{-16}$ for a single fibre of $100 \mathrm{~m}$ long.

\section{Field Programmable Gate Arrays}

Three FPGA technologies are used in the Level-0 trigger. They are characterized by the way they are configured:

- Anti-fuse based FPGAs (ACTEL AX family), that can be programmed only once;

- Flash-EEPROM based FPGAs (ACTEL pro-ASIC family), that can be erased and reprogrammed;

- RAM based FPGAs (Altera Acex, Flex, Stratix and Stratix GX families or Xilinx Virtex family) that can be reprogrammed an unlimited number of times.

Anti-fuse and flash FPGAs are used in the front-end boards close to the detector and are therefore exposed to significant radiation doses. These components have been tested in heavy ion beams and have shown very low sensitivity to single event upsets and single event latch-up. Special mechanisms such as triple-voting or horizontal and vertical parity are implemented to increase the protection of registers containing critical data. Dose effects begin to appear in Flash based FPGAs for doses an order of magnitude above the total dose received during 10 years by the trigger frontend electronics.

RAM-based FPGAs are known to be very sensitive to single event upsets. For this reason their use is restricted to boards located in the barracks which is a radiation free area.

All the FPGAs used in the trigger provide for good visibility of internal node behavior during the debug phase by providing embedded logic analyzer features (Silicon Explorer for ACTEL, SignalTap for the largest components of the Altera family and Chipscope for the Xilinx family).

\section{Debugging and monitoring tools}

Each Level-0 trigger board includes either a credit card PC or a SPECS component interfaced to the embedded FPGAs by a custom 16-bit bus. By this means the operation of any of the FPGAs is controlled and error detection mechanisms, such as error counters, spy and snooping mechanisms are implemented.

To test a complete sub-trigger in stand-alone mode, a data injection buffer to substitute input data is implemented. Results of the processing can be read back via the credit card PC at the output of dedicated SPY memories

The level-0 trigger is a very complex system. Any malfunctions can therefore be difficult to understand and interpret. At each stage the input and results of the processing are logged. In 


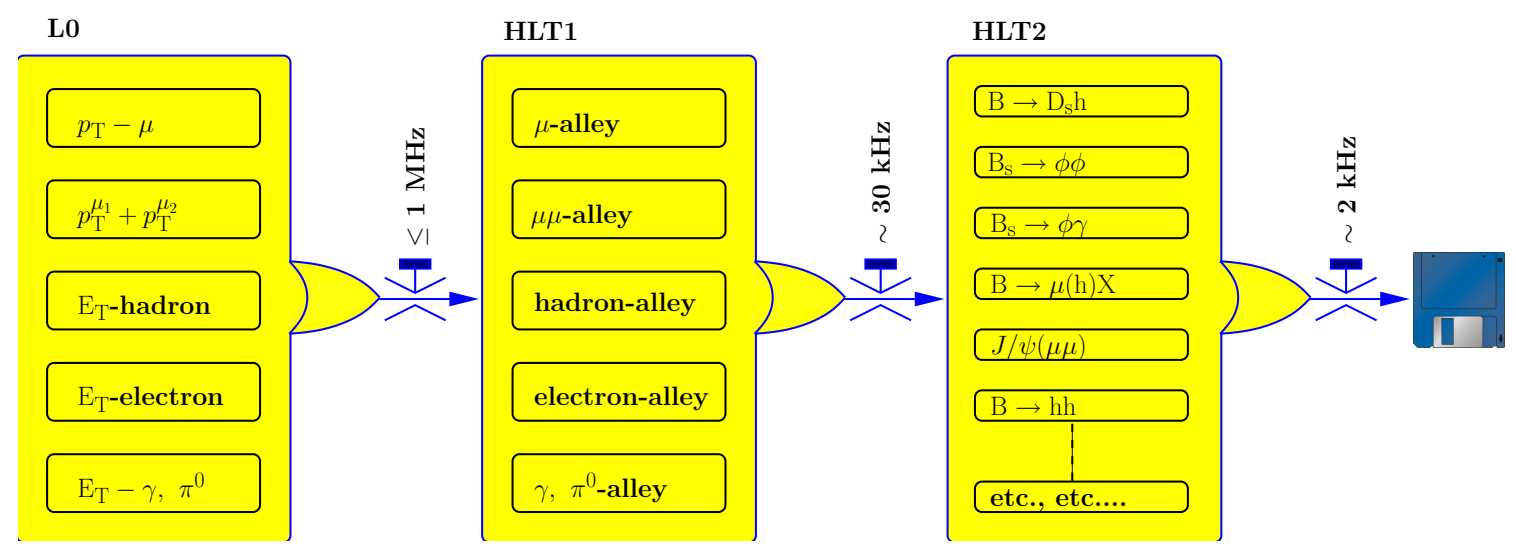

Figure 7.9: Flow-diagram of the different trigger sequences.

addition, a software emulator was developped which reproduces the behaviour of the hardware at the bit level. By comparing results computed by the hardware with those of the emulator run on the same input data, any faulty components can quickly be located.

\subsection{High Level Trigger}

The High Level Trigger (HLT) consists of a C++ application which runs on every CPU of the Event Filter Farm (EFF). The EFF contains up to 2000 computing nodes and is described in section 8. Each HLT application has access to all data in one event, and thus, in principle, could execute the off-line selection algorithms. However, given the $1 \mathrm{MHz}$ output rate of the Level-0 trigger and CPU power limitations, the HLT aims to reject the bulk of the uninteresting events by using only part of the full event data. In this section, the algorithm flow is described which, according to MonteCarlo simulation studies, is thought to give the optimal performance within the allowed time budget. However, it should be kept in mind that since the HLT is fully implemented in software, it is very flexible and will evolve with the knowledge of the first real data and the physics priorities of the experiment. In addition the HLT is subject to developments and adjustments following the evolution of the event reconstruction and selection software.

A schematic of the overall trigger flow is shown in figure 7.9. Level-0 triggers on having at least one cluster in the HCAL with $\mathrm{E}_{\mathrm{T}}^{\text {hadron }}>3.5 \mathrm{GeV}$, or the ECAL with $\mathrm{E}_{\mathrm{T}}^{\mathrm{e}, \gamma, \pi^{0}}>2.5 \mathrm{GeV}$, or a muon candidate in the muon chambers with $p_{\mathrm{T}}^{\mu}>1.2 \mathrm{GeV}$, or $p_{\mathrm{T}}^{\mu_{1}}+p_{\mathrm{T}}^{\mu_{2}}>1 \mathrm{GeV}$, where $\mu_{1}$ and $\mu_{2}$ are the two muons with the largest $p_{\mathrm{T}}$. The above thresholds are typical for running at a luminosity of $2 \times 10^{32} \mathrm{~cm}^{-2} \mathrm{~s}^{-1}$, but depend on luminosity and the relative bandwidth division between the different Level-0 triggers. All Level-0 calorimeter clusters and muon tracks above threshold are passed to the HLT as part of the Level-0 trigger information as described in section 7.1.2, and will be referred to as Level-0 objects henceforward.

The HLT is subdivided in two stages, HLT1 and HLT2. The purpose of HLT1 is to reconstruct particles in the VELO and T-stations corresponding to the Level-0 objects, or in the case of Level-0 $\gamma$ and $\pi^{0}$ candidates to confirm the absence of a charged particle which could be associated to these objects. This is called Level-0 confirmation, and the details of how this is achieved within the 
CPU time budget is explained below. HLT1 should reduce the rate to a sufficiently low level to allow for full pattern recognition on the remaining events, which corresponds to a rate of about 30 $\mathrm{kHz}$. At this rate HLT2 performs a combination of inclusive trigger algorithms where the B decay is reconstructed only partially, and exclusive trigger algorithms which aim to fully reconstruct Bhadron final states.

\subsection{HLT1}

HLT1 starts with so-called alleys, where each alley addresses one of the trigger types of the Level-0 trigger. About $\sim 15 \%$ of the Level- 0 events are selected by multiple triggers, and will consequently pass by more than one alley. To confirm the Level- 0 objects each alley makes use of the following algorithms:

$\mathrm{L} 0 \rightarrow \mathrm{T}$ : The Level-0 objects are assumed to originate from the interaction region, which defines the whole trajectory of the candidate in the spectrometer. So-called T-seeds are reconstructed in the T-stations, decoding only the hits in a window around the trajectory, or in case of the calorimeter clusters the two trajectories corresponding to the two charge hypothesis. The seeds are required to match the Level- 0 object in both space and momentum.

L0 $\rightarrow$ VELO: VELO-seeds are reconstructed in two stages. First the information from the $\mathrm{R}$-sensors are used to reconstruct $2 \mathrm{D}$-tracks. The $\chi^{2}$ is calculated for the matching of a 2D track with the Level-0 object, and only candidates with a sufficiently low $\chi^{2}$ are used to reconstruct a VELO-seed using the $\phi$-sensor information. These VELO-seeds in turn are required to match the Level-0 object with a sufficiently small $\chi^{2}$. In addition the 2D-tracks are used to reconstruct the primary vertexes in the event [216].

$\mathrm{VELO} \rightarrow \mathrm{T}$ : The VELO-seeds above define a trajectory in the T-stations, around which a Tseed is reconstructed completely analogue to the $\mathrm{L} 0 \rightarrow \mathrm{T}$ algorithm described above.

$\mathrm{T} \rightarrow$ VELO: this algorithm finds the VELO-seeds which match a T-seed, using an algorithm analogue to the $\mathrm{L} 0 \rightarrow$ VELO algorithm, but now starting from a T-seed, rather than a Level-0 object.

Each HLT1 alley uses a sequence of the above algorithms to reduce the rate. An algorithm common to all alleys is used for computing the primary vertex with the $2 \mathrm{D}$ tracks reconstructed in the VELO. While the alleys are operating independently, care has been taken to avoid having to reconstruct the same track or primary vertex twice to avoid wasting precious CPU power.

While the bandwidth division between the alleys has not been defined, the performance of the alleys will be illustrated with two typical alleys, the muon and hadron alleys running at a luminosity of $2 \times 10^{32} \mathrm{~cm}^{-2} \mathrm{~s}^{-1}$.

The HLT1 $\mu$-alley input rate will be $\sim 230 \mathrm{kHz}$, and contain $1.2 \mathrm{~L}^{\mu}$ objects per event. $\mathrm{L}^{\mu} \rightarrow \mathrm{T}$ reduces the rate to $120 \mathrm{kHz}$, while the number of candidates increases to $1.8 \mathrm{~T}$-seeds per event. $\mathrm{T} \rightarrow \mathrm{VELO}$ reduces the rate to $80 \mathrm{kHz}$. Requiring the remaining candidates to have an impact parameter to any primary vertex larger than $0.1 \mathrm{~mm}$ reduces the rate to $10 \mathrm{kHz}$. The HLT1 hadronalley input rate will be $\sim 600 \mathrm{kHz}$, and contain $1.3 \mathrm{~L} 0^{\text {hadron }}$ objects per event. L $0^{\text {hadron }} \rightarrow$ VELO, requiring a $0.1 \mathrm{~mm}$ impact parameter of the VELO-seeds to any primary vertex reduces the rate to $300 \mathrm{kHz}$ which contain 2.2 VELO-seeds per event. VELO $\rightarrow$ T reduces this rate to $30 \mathrm{kHz}$ with 1.2 candidates per event. Since this rate is still too large for the HLT2 stage, a further reduction is obtained by requiring a VELO-track with a distance of closest approach to the confirmed Level-0 
track of less than $0.2 \mathrm{~mm}$, and a $p_{\mathrm{T}}$ of at least $1 \mathrm{GeV}$. This reduces the rate to $11 \mathrm{kHz}$ with 3.2 candidate secondary vertices per event. The other HLT1 alleys employ similar strategies.

\subsection{HLT2}

The combined output rate of events accepted by the HLT1 alleys is sufficiently low to allow an off-line track reconstruction as described in section 10.1. The HLT-tracks differ from the off-line in not having been fitted with a Kalman filter to obtain a full covariance matrix since this is too CPU intensive. Prior to the final selection, a set of tracks is selected with very loose cuts on their momentum and impact parameter. These tracks are used to form composite particles, such as $\mathrm{K}^{*} \rightarrow \mathrm{K}^{+} \pi^{-}, \phi \rightarrow \mathrm{K}^{+} \mathrm{K}^{-}, \mathrm{D}^{0} \rightarrow \mathrm{hh}, \mathrm{D}_{\mathrm{s}} \rightarrow \mathrm{K}^{+} \mathrm{K}^{-} \pi^{-}$and $\mathrm{J} / \psi \rightarrow \mu^{+} \mu^{-}$, which are subsequently used for all selections to avoid duplication in the creation of final states.

The HLT2 stage uses therefore cuts either on invariant mass, or on pointing of the B momentum towards the primary vertex. The resulting inclusive and exclusive selections aim to reduce the rate to about $2 \mathrm{kHz}$, the rate at which the data is written to storage for further analysis. The exclusive triggers are sensitive to tracking performance, while the inclusive triggers select partial $\mathrm{B}$ decays to $\phi \mathrm{X}, \mathrm{J} / \psi \mathrm{X}, \mathrm{D}^{*} \mathrm{X}, \mu^{ \pm} \mathrm{X}, \mu^{ \pm} \mathrm{hX}$ and $\mu^{+} \mu^{-} \mathrm{X}$ and therefore are less dependent on the on-line reconstruction. However, the exclusive selection of these channels produces a smaller rate, thus allowing for a more relaxed set of cuts. The final trigger is the logical OR of the inclusive and exclusive selections.

\subsection{HLT monitoring}

Each HLT1 alley and HLT2 selection produces summary information which is written to storage for the accepted events. This summary contains the information of all tracks and vertexes which triggered the event. It is foreseen to reserve a significant fraction of the output bandwidth for triggers on semi-leptonic B-decays, hence a sample in which the trigger did not bias the decay of the accompanying B-hadron. The summary information is used to check if an event would have triggered, even if the B decay of interest would not have participated in the trigger. It therefore allows to study the trigger performance. The summary information also guarantees that during the analysis the trigger source of an individual event is known.

To assure that during off-line analysis the trigger conditions are known, the combination of trigger algorithms with their selection parameters will be assigned a unique key, the Trigger Configuration Key (TCK). All trigger configurations with their associated TCK are pre-loaded in the EFF before a fill. To change from one trigger configuration to another one, for example to follow the decaying luminosity in a fill, a new TCK must be selected. This TCK is attached by the Time and Fast Control system (TFC, see section 8.3) to each event, and it steers the configuration of the algorithms on the EFF and allows full traceability of the used configuration. 


\section{Chapter 8}

\section{Online System}

The task of the Online system is to ensure the transfer of data from the front-end electronics to permanent storage under known and controlled conditions. This includes not only the movement of the data themselves, but also the configuration of all operational parameters and the monitoring of these, as well as environmental parameters, such as temperatures or pressures. The online system also must ensure that all detector channels are properly synchronized with the LHC clock. The LHCb Online system is described in detail in [214, 217, 218]

\subsection{System decomposition and architecture}

The LHCb Online system consists of three components:

- the Data Acquisition (DAQ) system,

- the Timing and Fast Control (TFC) system,

- the Experiment Control System (ECS).

The general architecture of the LHCb online system is shown in figure 8.1.

\subsection{Data Acquisition System}

The purpose of the Data Acquisition (DAQ) system is the transport of the data belonging to a given bunch crossing, and identified by the trigger, from the detector front-end electronics to permanent storage. The design principles for the DAQ architecture (figure 8.1) are:

- Simplicity: simple protocols and a small number of components with simple functionalities

- Scalability: ability to react to changing system parameters, such as event sizes, trigger rates or the CPU needs of trigger algorithms.

- Only point-to-point links: components are connected through point-to-point links only. No buses are used (outside monolithical boards). This leads to a more robust system. 


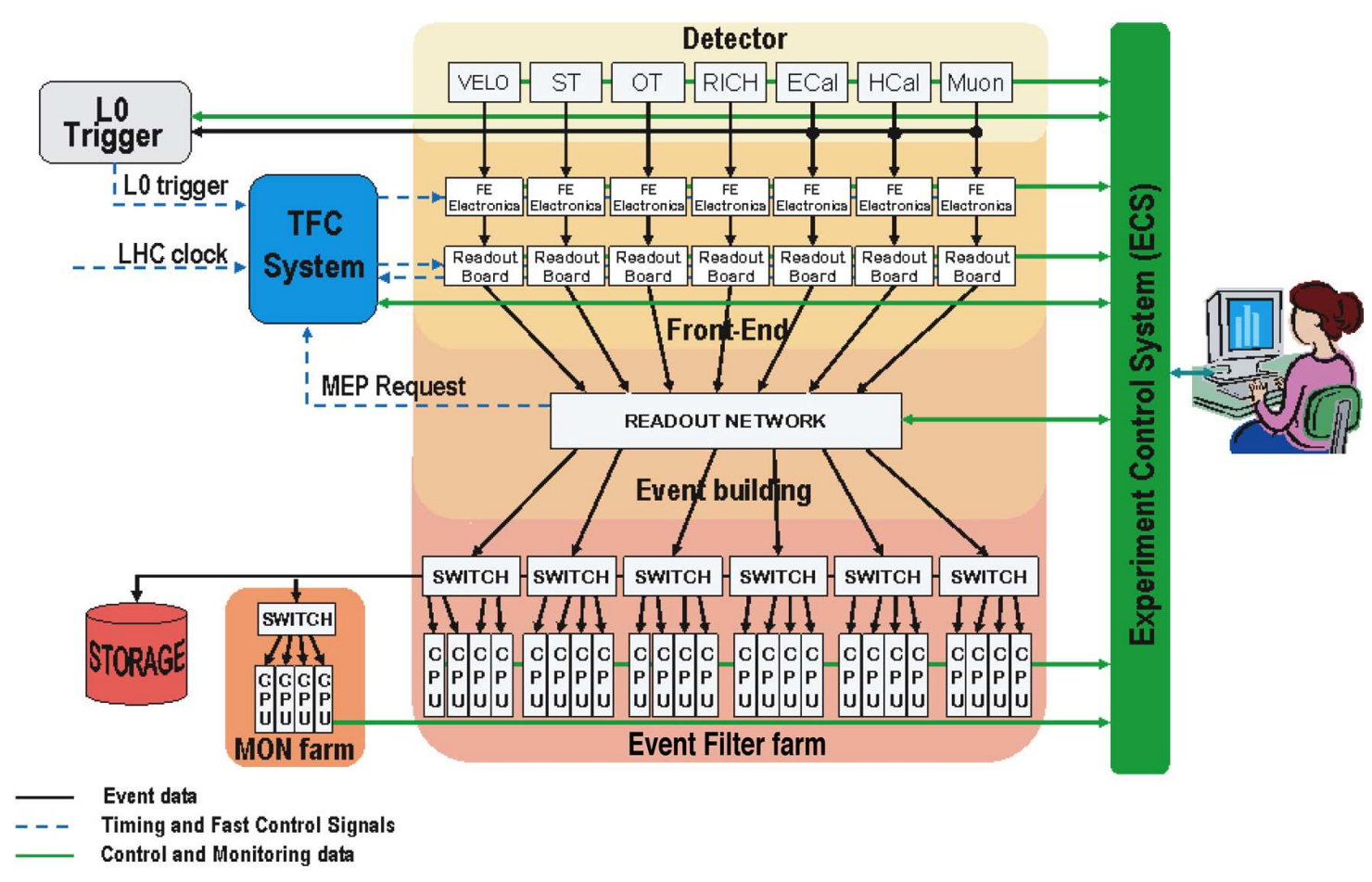

Figure 8.1: General architecture of the LHCb Online system with its three major components: Timing and Fast Controls, Data Acquisition and Experiment Control System. A blow-up of the the TFC box is shown in figure 8.3.

- Use of Commercial off-the-shelf (COTS) products and, wherever possible, commodity components and protocols.

These principles allowed to construct a reliable and robust system with enough flexibility to cope with possible new requirements, motivated by experience with real data.

Data from the on/near-detector electronics (front-end electronics) are collected in LHCb-wide standardized readout boards (TELL1). ${ }^{1}$ Figure 8.2 shows a simplified block diagram of the TELL1 board. A detailed description can be found in [10].

Data are received from the detector electronics either by optical or analogue receiver cards and processed in four pre-processing FPGAs, ${ }^{2}$ where common-mode processing, zero-suppression or data compression is performed depending on the needs of individual detectors. The resulting data fragments are collected by a fifth FPGA (SyncLink) and formatted into a raw IP-packet that is subsequently sent to the DAQ system via the 4-channel GbEthernet mezzanine card. The board interfaces to the Experiment Control System (ECS) by means of a credit-card sized PC mounted on the board. Clock and synchronization signals (e.g. triggers) are transmitted through the on-board Trigger, Timing and Control (TTC) interface [219]. Flow control to the TFC system is performed through the throttle signal driven by the SyncLink FPGA.

\footnotetext{
${ }^{1}$ The RICH detectors use a specific board (c.f. 6.1) which is, from the data readout point of view, functionally identical to the TELL1.

${ }^{2}$ Altera Stratix $1 \mathrm{~S} 25$.
} 


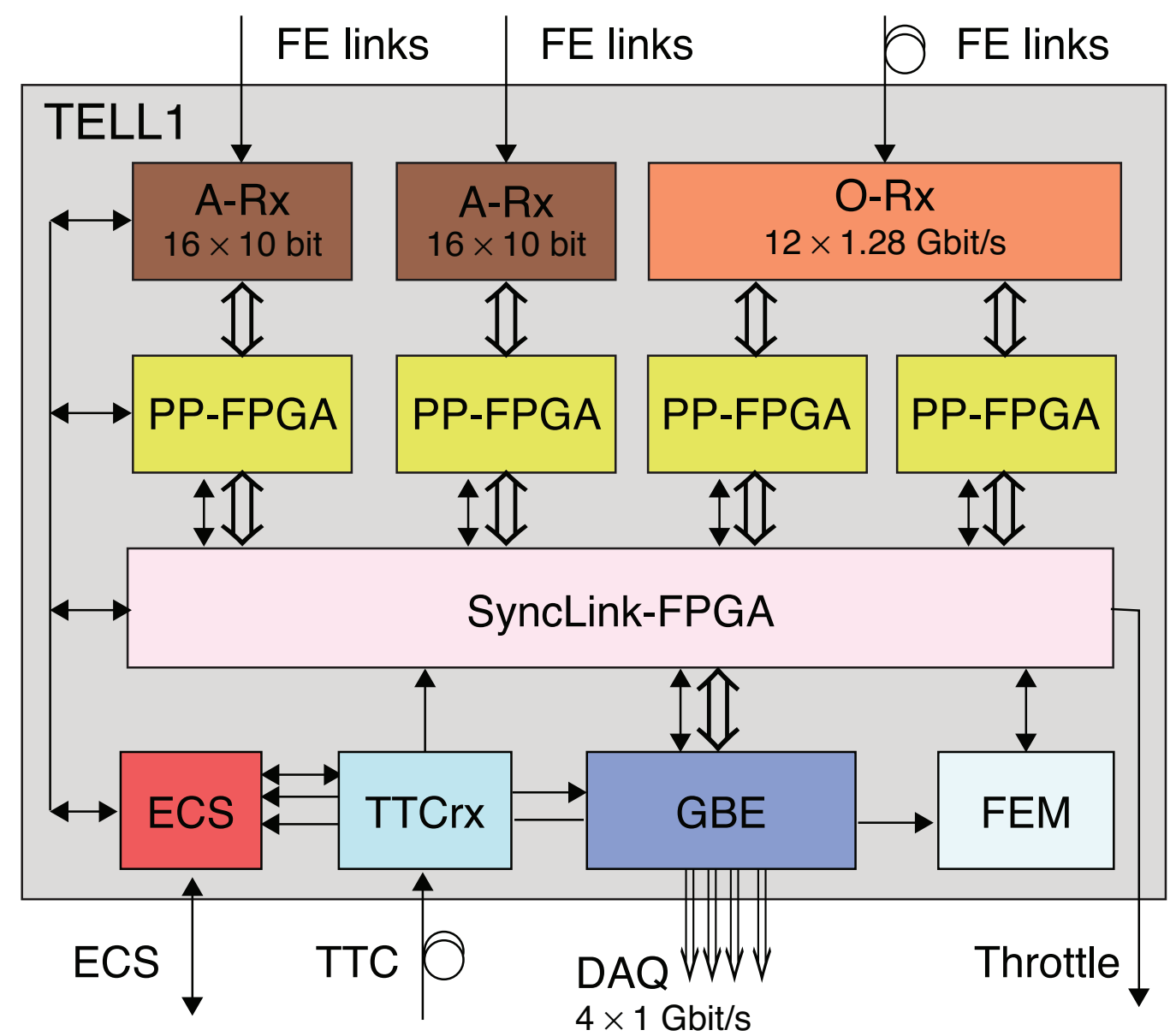

Figure 8.2: Simplified block diagram of the common readout board TELL1. The FEM (Front-End Multiplexer) allows to merge event fragments from several input links to form one output fragment.

In the CPU farm, the HLT algorithm selects interesting interactions; upon a positive decision, the data are subsequently sent to permanent storage. The HLT (see 7.2) is expected to reduce the overall rate from the original trigger rate of $1 \mathrm{MHz}$ to $\sim 2 \mathrm{kHz}$, hence by a factor of 500 . The storage system is expected to have a capacity of $\sim 40 \mathrm{~TB}$, which should offer sufficient buffer space to cope with possible interruptions of the transfer to permanent storage at CERN. Gigabit-Ethernet was chosen as link technology, mainly because of its wide, almost monopoly-like, acceptance in the LAN market and its low price. The very wide range of speed from $10 \mathrm{Mb} / \mathrm{s}$ to $10 \mathrm{~Gb} / \mathrm{s}$, and the availability of very big switches ( $>1200$ ports per chassis) are also important assets.

The TELL1 board offers $4 \mathrm{~Gb}$ Ethernet ports as output stages. Some of these are fed into a large switching network providing the connectivity between the TELL1 boards and the individual Farm nodes. To overcome the significant overhead per frame of Ethernet, the concept of MultiEvent Packets has been devised, in which the data of several triggers $(\sim 10)$ are collected in one IP packet and transferred subsequently through the network. The size of the CPU farm running the HLT trigger algorithms is determined by the average execution time of the HLT algorithm per event but also possibly by the maximum bandwidth into an individual processing node: if the execution 
time were to be very low, the input bandwidth might constitute the limiting factor and the number of boxes would have to be increased. The HLT algorithms are executed on a sizeable farm of CPUs. It's is expected to consist of 1000-2000 1U servers containing CPUs with multi-core technologies. The starting size of the farm will be about 200 servers. The maximum available space is $2200 \mathrm{U}$. The large number of CPUs is organized into 50 sub-farms of 20-40 CPUs each. The scalability is then guaranteed since one sub-farm is a functional unit and there is no cross-communication between sub-farms.

The quality of the acquired data is checked in a separate monitoring farm that will receive events accepted by the HLT and will house user-defined algorithms to determine e.g. the efficiencies of detector channels or the mass resolution of the detector. Also, some rate of L0 accepted and random triggers will be used to monitor the trigger itself.

\subsection{Timing and Fast Control}

The TFC system drives all stages of the data readout of the LHCb detector between the front-end electronics and the online processing farm by distributing the beam-synchronous clock, the L0 trigger, synchronous resets and fast control commands. The system is a combination of electronic components common to all LHC experiments and LHCb custom electronics. The TFC architecture shown in figure 8.3 can be described in terms of three main ingredients, the TFC distribution network, the trigger throttle network, and the TFC master (Readout Supervisor).

The TFC optical distribution network with transmitters and receivers is based on the LHCwide TTC system developed at CERN [219]. In addition to transmitting the beam synchronous clock, the protocol features a low-latency trigger channel and a second channel with framed user data used to encode the control commands. A switch has been developed and introduced into the distribution network to allow a dynamic partitioning of the LHCb detector to support independent and concurrent sub-detector activities such as commissioning, calibration and testing.

The optical throttle network is used to transmit back-pressure, that is a trigger inhibit, from the asynchronous parts of the readout system to the Readout Supervisor in case of congestion of the data path. The network incorporates a Throttle Switch to support the requirement that the readout system is partitionable, and to allow modules to perform an $O R$ of the throttle signals of each sub-system locally.

The heart of the system, the Readout Supervisor, implements the interface between the LHCb trigger system and the readout chain. The Readout Supervisor synchronizes trigger decisions and beam-synchronous commands to the LHC clock and orbit signal provided by the LHC. It is also capable of producing a variety of auto-triggers for sub-detector calibration and tests, and performs the trigger control as a function of the load on the readout system. In order to perform dynamic load balancing among the nodes in the online processing farm, the Readout Supervisor also selects and broadcasts the destination for the next set of events to the Readout Boards based on a credit scheme in which the farm nodes send data requests directly to the Readout Supervisor.

For each trigger the Readout Supervisor transmits a data bank over the readout network which is appended to the event data and which contains the identifier of the event, the time and the source of the trigger. 


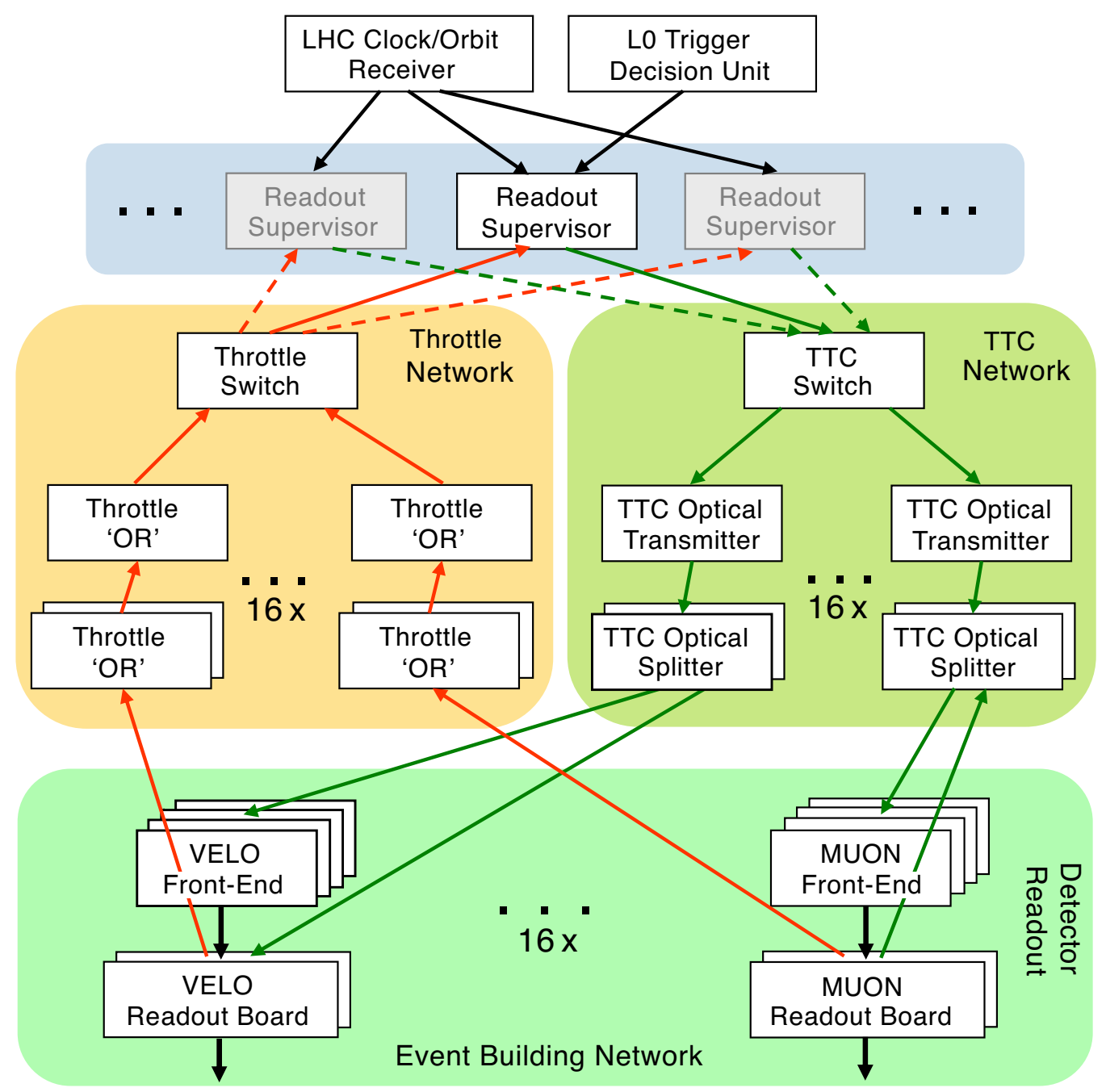

Figure 8.3: Schematic diagram of the TFC architecture. For a given partition there is only one RS, but several partitions can operate at the same time.

\subsection{Experiment Control System}

The Experiment Control System (ECS) ensures the control and monitoring of the operational state of the entire $\mathrm{LHCb}$ detector. This encompasses not only the traditional detector control domains, such as high and low voltages, temperatures, gas flows, or pressures, but also the control and monitoring of the Trigger, TFC, and DAQ systems. The hardware components of the ECS are somewhat diverse, mainly as a consequence of the variety of the equipment to be controlled, ranging from standard crates and power supplies to individual electronics boards. In LHCb, a large effort was made to minimize the number of different types of interfaces and connecting busses. The field busses have been restricted to:

- SPECS, Serial Protocol for ECS, a serial bus providing high-speed, 10Mb/s, control access to front-end electronics [41], 


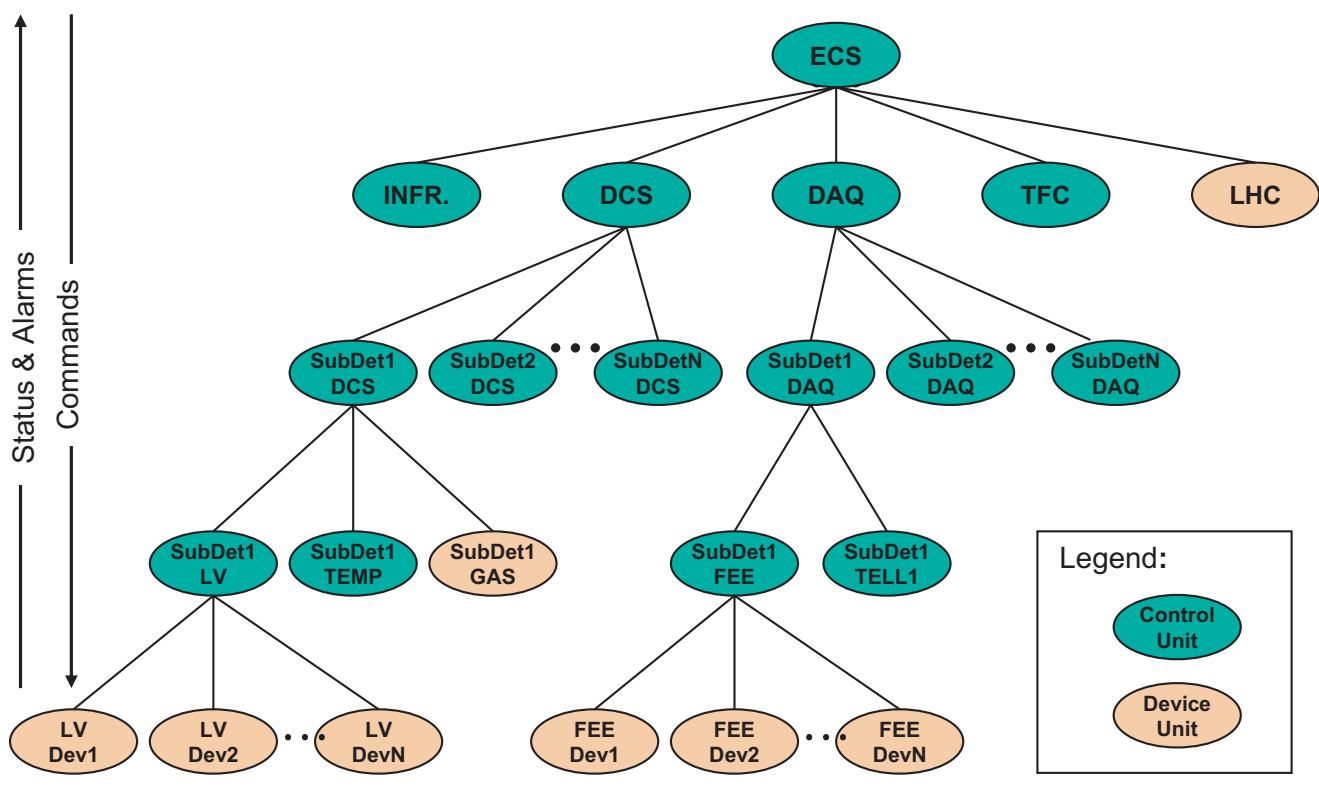

Figure 8.4: ECS architecture.

- CAN (Controller Area Network ${ }^{3}$ ),

- (fast)Ethernet.

The first two, SPECS and CAN, are mainly used for equipment residing in the high radiation area close to the detector. The associated interfaces tolerate modest levels of radioactivity but are not radiation hard. Ethernet is only used in the radiation free areas, such as the electronics barracks or on the surface. Ethernet is used to control individual PCs, as well as the individual electronics boards usedfor the readout through Credit-Card sized PCs mounted directly on each board. This choice allows the use of normal PCs over their standard Ethernet interfaces for controlling the readout electronics.

The ECS software is based on PVSS II, a commercial SCADA (Supervisory Control And Data Acquisition) system. This toolkit provides the infrastructure needed for building the ECS system, such as a configuration database and communication between distributed components, graphical libraries to build operations panels, and an alarm system as well as components, such as OPC clients. Based on PVSS, a hierarchical and distributed system was designed as depicted in figure 8.4.

Device Units, in figure 8.4, denote low-level access components which model the physical device and typically communicate directly with the hardware. In general they only implement a very simple state machine which is exclusively driven by the controlling Control Unit. Examples of Device Units are power supplies, and software processes, such as the HLT processes.

Control Units implement high-level states and transitions and also local logic to support recovery from errors of subordinate Device Units. Typical examples of Control Units are a HV subsystem, or the component that controls the ensemble of crates of a sub-detector or an entire

${ }^{3}$ ISO Standard 11898, see e.g. www.iso.org. 
sub-farm of the Event Filter Farm. Control Units can be controlled by other Control Units, to allow the building of a hierarchy of arbitrary depth. State sequencing in the ECS system is achieved using a Finite State Machine package, based on SMI++ that allows creating complex logic needed, for example, for implementing elaborate sequencing or automatic error recovery.

The distributed components of the ECS system are connected with a large Ethernet network consisting of several hundred Gigabit and Fast Ethernet links. 


\section{Chapter 9}

\section{Computing and Resources}

This section describes the dataflow of the LHCb computing model for all stages in the processing of the real and simulated LHCb events [22]. The roles of the various Tier centres are discussed and the distribution of the processing load and storage are outlined.

There are several phases in the processing of event data. The various stages normally follow each other in a sequential manner, but some stages may be repeated a number of times. The workflow presented here reflects the present understanding of how to process the data. A schematic of the logical dataflow is shown in figure 9.1 and is described in more detail below.

The raw data from the detector is produced via the Event Filter farm of the online system. The first step is to collect data, triggering on events of interest. The raw data are transferred to the CERN Tier 0 centre for further processing and archiving. The raw data, whether real or simulated, must then be reconstructed to form physical quantities such as the calorimeter clusters needed to provide the energy of electromagnetic and hadronic showers, tracker hits to be associated to tracks whose position and momentum are to be determined. Information about particle identification (electron, photon, $\pi^{0}$, charged hadrons, muon) is also reconstructed from the appropriate sub-systems. The

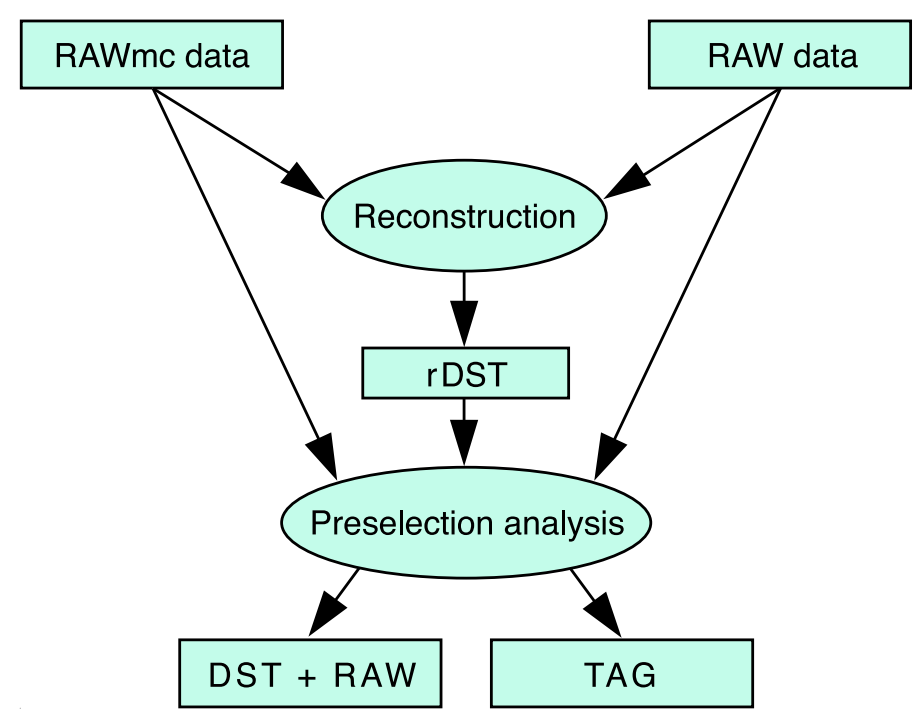

Figure 9.1: The LHCb computing logical dataflow model. 
event reconstruction results in the generation of new data, the Data Summary Tape (DST). Only enough data will be stored in the DST to allow the physics pre-selection algorithms to be run at the next stage: this is known as a reduced DST (rDST). The first pass of the reconstruction will happen in quasi-real time. It is planned to reprocess the data of a given year once, after the end of data taking for that year, and then periodically as required. This is to accommodate improvements in the reconstruction algorithms and to make use of improved determinations of the calibration and alignment of the detector in order to generate new improved rDST information.

The rDST is analysed in a production-type mode in order to produce streams of selected events for further individual analysis. This activity is known as stripping. The rDST information is used to determine the four-momentum vectors corresponding to the measured particles, to locate primary and secondary vertices and reconstruct composite particles such as B candidates. A preselection algorithm will be provided for each channel of interest. Since these algorithms use tools that are common to many different physics analyses they are run in production-mode as a first step in the analysis process. The events that pass the selection criteria will be fully re-reconstructed, recreating the full information associated with each event. The output of the stripping stage will be referred to as the (full) DST and contains more information than the rDST. Before being stored, the events that pass the selection criteria will have their raw data added in order to have as detailed event information as needed for the analysis. An event tag collection will also be created for faster reference to selected events. The tag contains a brief summary of each event's characteristics as well as the results of the pre-selection algorithms and a reference to the actual DST record. The event tags are stored in files independent of the actual DST files. It is planned to run this productionanalysis phase 4 times per year: once with the original data reconstruction; once with the reprocessing of the raw data, and twice more, as the selection cuts and analysis algorithms evolve.

The baseline LHCb computing model is based on a distributed multi-tier regional centre model. It attempts to build in flexibility that will allow effective analysis of the data whether the Grid middleware meets expectations or not. A schematic of the LHCb computing model is given in figure 9.2.

CERN is the central production centre and will be responsible for distributing the raw data in quasi-real time to the Tier-1 centres. CERN will also take on the role of a Tier- 1 centre. Six additional Tier-1 centres have been identified: CNAF (Italy), FZK (Germany), IN2P3 (France), NIKHEF (The Netherlands), PIC (Spain) and RAL (United Kingdom): there is also a number of Tier- 2 computing centres. CERN and the Tier- 1 centres will be responsible for all the productionprocessing phases associated with the real data. The raw data will be stored in its entirety at CERN, with another copy distributed across the other 6 Tier- 1 centres. The second pass of the full reconstruction of the raw data will also use the resources of the LHCb online farm. As the production of the stripped DSTs will occur at these computing centres, it is envisaged that the majority of the distributed analyses will be performed at CERN and at the Tier- 1 centres. The current year's stripped DST will be distributed to all centres to ensure load balancing. It should be noted that although no user analysis is envisaged at the Tier- 2 centres in the baseline model presented, it should not be proscribed, particularly for the larger Tier- 2 centres.

It is expected that the reconstruction and the first stripping of data at CERN and at the Tier-1 centres will follow the production in quasi real-time, with a maximum delay of a few days. The DST output of the stripping will remain on disk for analysis and be distributed to all other Tier-1 centres and CERN, whilst the raw and rDST will be migrated to the mass storage system, MSS. 


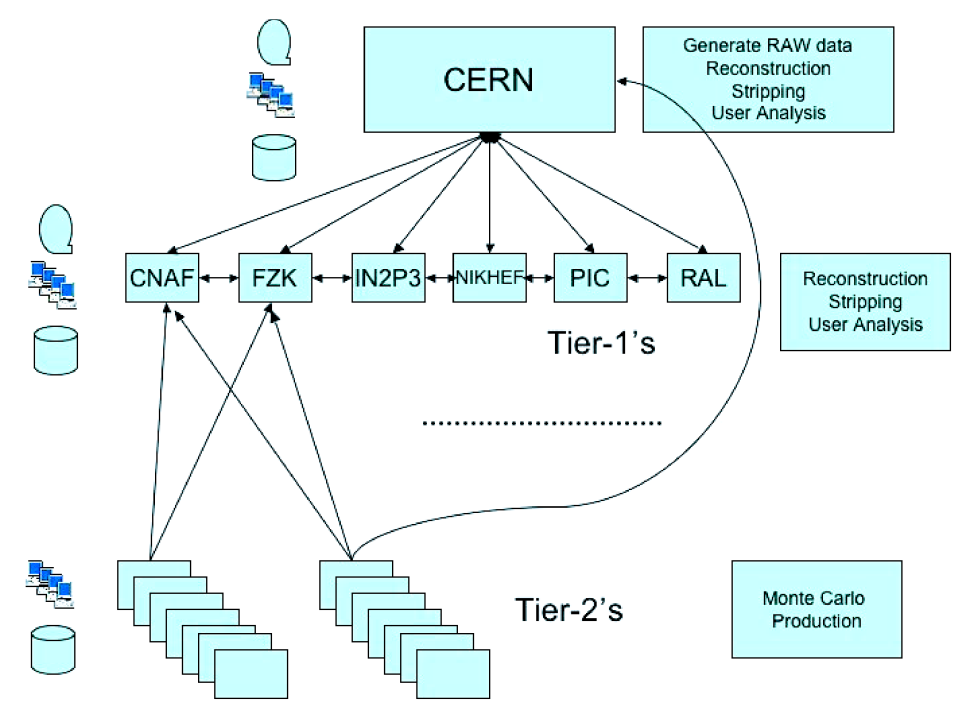

Figure 9.2: Schematic of the LHCb Computing Model.

Table 9.1: Projected resource usage in 2008 at CERN, the Tier-1 and Tier- 2 centres. A 1 GHz PIII processor is equivalent to $400 \mathrm{KSI} 2 \mathrm{k}$.

\begin{tabular}{|c|c|c|c|}
\hline Site & CPU (MSI2k.years) & Disk (TB) & Tape (TB) \\
\hline \hline CERN & 0.36 & 350 & 631 \\
\hline Tier-1 & 1.77 & 1025 & 860 \\
\hline Tier-2 & 4.55 & - & - \\
\hline
\end{tabular}

The re-processing of the data will occur over a two-month period. During this process the raw data will need to be accessed from the MSS both at CERN and the Tier- 1 centres. The CPU resources available at the pit allow a significant fraction of the total re-processing and perhaps the subsequent stripping to be performed there. Hence at CERN there is an additional complication that the raw data will also have to be transferred to the pit; similarly, the produced rDST will have to be transferred back to the CERN computing centre. To enable later stripping, it is necessary to distribute a fraction of the rDST produced at CERN during this re-processing to the Tier- 1 centres; this is a consequence of the large contribution from the online farm.

The (two) stripping productions outside of the reconstruction or of the re-processing of the data will be performed over a one-month period. Both the raw and the rDST will need to be accessed from the MSS to perform this production. The produced stripped DSTs will be distributed to all production centres.

The Tier- 2 centres will be primarily MonteCarlo production centres, with both CERN and the Tier- 1 centres acting as the central repositories for the simulated data. The MonteCarlo production is expected to be an ongoing activity throughout the year and is the mainstay of the Tier- 2 centres. The whole of the current year's MonteCarlo production DST will be available on disk at CERN and another 3 copies, on disk, distributed amongst the other 6 Tier- 1 centres.

The 2008 resource requirements needed for the LHCb computing model at CERN and integrated across the Tier- 1 centres and the Tier- 2 centres are given in table 9.1. 


\section{Chapter 10}

\section{Performance}

The data used to study the performance of the $\mathrm{LHCb}$ detector has been obtained from detailed MonteCarlo simulation, which produces a raw data format identical to real data. The proton-proton collisions are simulated with the PYTHIA program [220]. The generated particles are tracked through the detector and the surrounding material using the GEANT package [221]. The geometry and material composition of the LHCb detector are described in detail. The detector response, resolution, noise, crosstalk, etc. have been tuned to comply with test beam results. Details about this can be found in [2].

The performance quoted in this chapter is only qualitative, for two main reasons: 1) the MonteCarlo estimations are being constantly refined due to better and more detailed description of the detector, its material, the response of the electronics, more realistic source of noise, and also due to improvements in GEANT, and 2) more importantly, the real performance will be established and understood only using the first collected data as LHC will collide beams.

In the following, a description of the procedures for the main LHCb detector tasks, i.e. tracking, vertex reconstruction, particle identification, mass reconstruction, is given.

\subsection{Track reconstruction}

In the track reconstruction software the hits in the VELO, the TT, the IT and the OT detectors are combined to form particle trajectories from the VELO to the calorimeters. The reconstruction algorithm aims to find all tracks in the event which leave sufficient detector hits, and not only those from b-hadron decay.

Depending on their trajectories inside the spectrometer the following classes of tracks are defined, illustrated in figure 10.1:

- Long tracks, traversing the full tracking set-up from the VELO to the T stations. These have the most precise momentum determination and therefore are the most important set of tracks for b-hadron decay reconstruction.

- Upstream tracks, traversing only the VELO and TT stations. These are in general lower momentum tracks that are bent out of the detector acceptance by the magnetic field. However, they pass through the RICH 1 detector and may generate Cherenkov photons if they have 


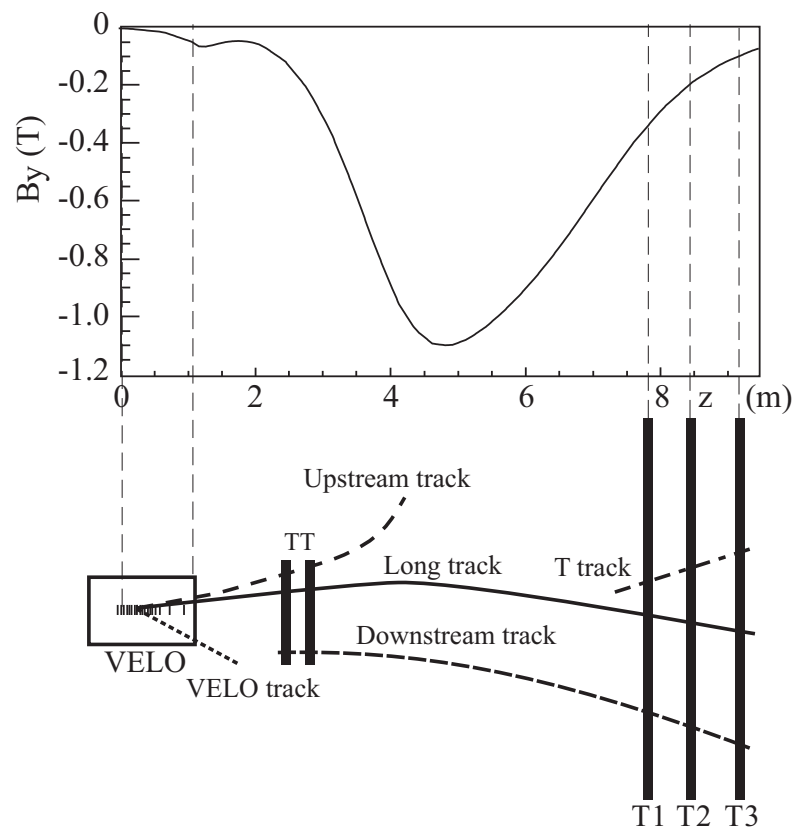

Figure 10.1: A schematic illustration of the various track types: long, upstream, downstream, VELO and T tracks. For reference the main $B$-field component $\left(B_{y}\right)$ is plotted above as a function of the $z$ coordinate.

velocities above threshold. They are therefore used to understand backgrounds in the RICH particle identification algorithm. They may also be used for b-hadron decay reconstruction or flavour tagging, although their momentum resolution is rather poor.

- Downstream tracks, traversing only the TT and T stations. The most relevant cases are the decay products of $\mathrm{K}_{\mathrm{S}}^{0}$ and $\Lambda$ that decay outside the VELO acceptance.

- VELO tracks, measured in the VELO only and are typically large angle or backward tracks, useful for the primary vertex reconstruction.

- T tracks: are only measured in the $\mathrm{T}$ stations. They are typically produced in secondary interactions, but are useful for the global pattern recognition in RICH 2.

The track reconstruction starts with a search for track seeds, the initial track candidates [222], in the VELO region and the $\mathrm{T}$ stations where the magnetic field is low. After tracks have been found, their trajectories are refitted with a Kalman filter [223] which accounts for multiple scattering and corrects for $d E / d x$ energy loss. The quality of the reconstructed tracks is monitored by the $\chi^{2}$ of the fit and the pull distribution of the track parameters.

The pattern recognition performance is evaluated in terms of efficiencies and ghost rates. The efficiencies are normalized to the reconstructible track samples. To be considered reconstructible, a track must have a minimum number of hits in the relevant subdetectors. To be considered as successfully reconstructed, a track must have at least $70 \%$ of its associated hits originating from a single MonteCarlo particle. The reconstruction efficiency is defined as the fraction of reconstructible tracks that are successfully reconstructed, and the ghost rate is defined as the fraction of 


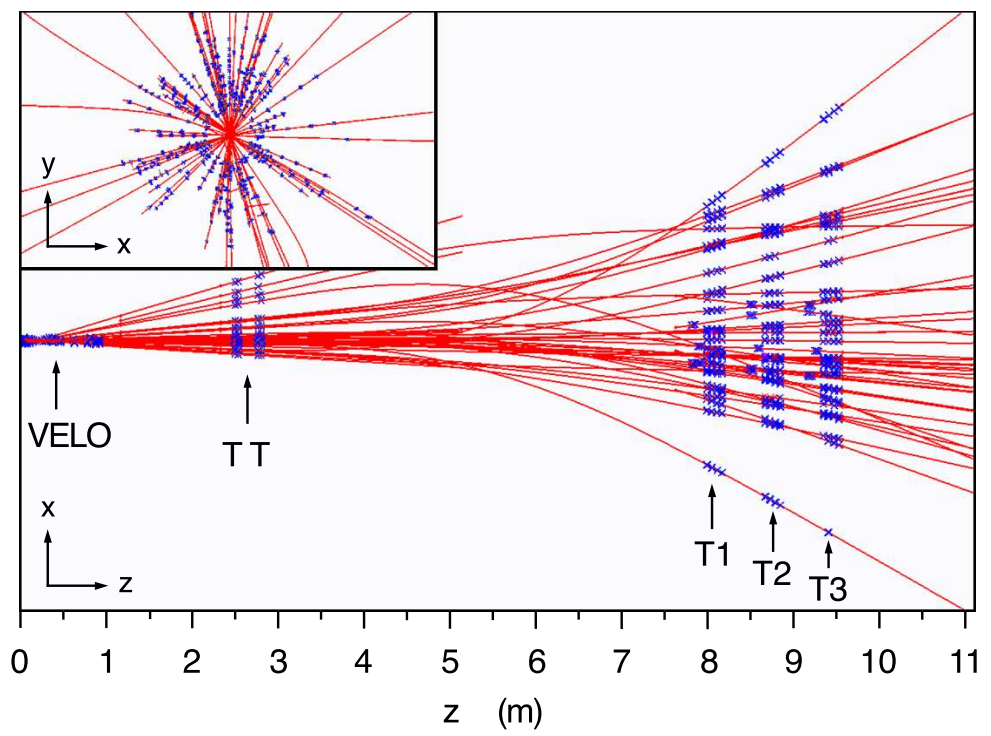

Figure 10.2: Display of the reconstructed tracks and assigned hits in an event. The insert shows a zoom in the plane $(x, y)$ into the VELO region.

reconstructed tracks that are not matched to a true MonteCarlo particle. The results quoted in this section are obtained from a sample of $\mathrm{B}^{0} \rightarrow \mathrm{J} / \psi \mathrm{K}_{\mathrm{S}}^{0}$ events.

An example of a reconstructed event is displayed in figure 10.2. The average number of successfully reconstructed tracks in fully simulated $b \bar{b}$ events is about 72 , which are distributed among the track types as follows: 26 long tracks, 11 upstream tracks, 4 downstream tracks, 26 VELO tracks and $5 \mathrm{~T}$ tracks. The track finding performance is summarized in the following for the most important cases: the long tracks, the low momentum (upstream) tracks and $\mathrm{K}_{\mathrm{S}}^{0}$ decay (downstream) tracks.

The efficiency to find as a long track the trajectory of a particle with momentum larger than $10 \mathrm{GeV} / c$ is on average $\sim 94 \%$. The corresponding average ghost fraction is about $9 \%$, but most ghost tracks have a low reconstructed $p_{\mathrm{T}}$.

The momentum and impact parameter resolutions of the reconstructed long tracks are shown in figure 10.3. The momentum resolution is plotted as a function of the track momentum and is seen to be increasing from $\delta p / p=0.35 \%$ for low momentum tracks to $\delta p / p=0.55 \%$ for tracks at the high end of the spectrum. In the same figure the momentum spectrum for B decay tracks is also illustrated. The impact parameter resolution is plotted as function of $1 / p_{\mathrm{T}}$ of the track. The linear dependence can be parametrized as $\sigma_{\mathrm{IP}}=14 \mu \mathrm{m}+35 \mu \mathrm{m} / p_{\mathrm{T}}$ with $p_{\mathrm{T}}$ in $\mathrm{GeV} / c$. For comparison the $1 / p_{\mathrm{T}}$ spectrum of $\mathrm{B}$ decay particles in the detector acceptance is plotted in the same figure.

The efficiency of the upstream track finding for particles with $p>1 \mathrm{GeV} / \mathrm{c}$ is approximately $75 \%$ with a corresponding ghost rate of $15 \%$. The momentum resolution is only $\delta p / p \sim 15 \%$, due to the small value of the total magnetic field integral in the considered region.

The efficiency for finding downstream tracks above $5 \mathrm{GeV} / \mathrm{c}$ is about $80 \%$. Since the downstream tracks traverse most of the magnetic field, the momentum resolution is relatively good with an average of $\delta p / p=0.43 \%$ for pions originating from $\mathrm{K}_{\mathrm{S}}^{0}$ decays in $\mathrm{B}^{0} \rightarrow \mathrm{J} / \psi \mathrm{K}_{\mathrm{S}}^{0}$ events. In order 

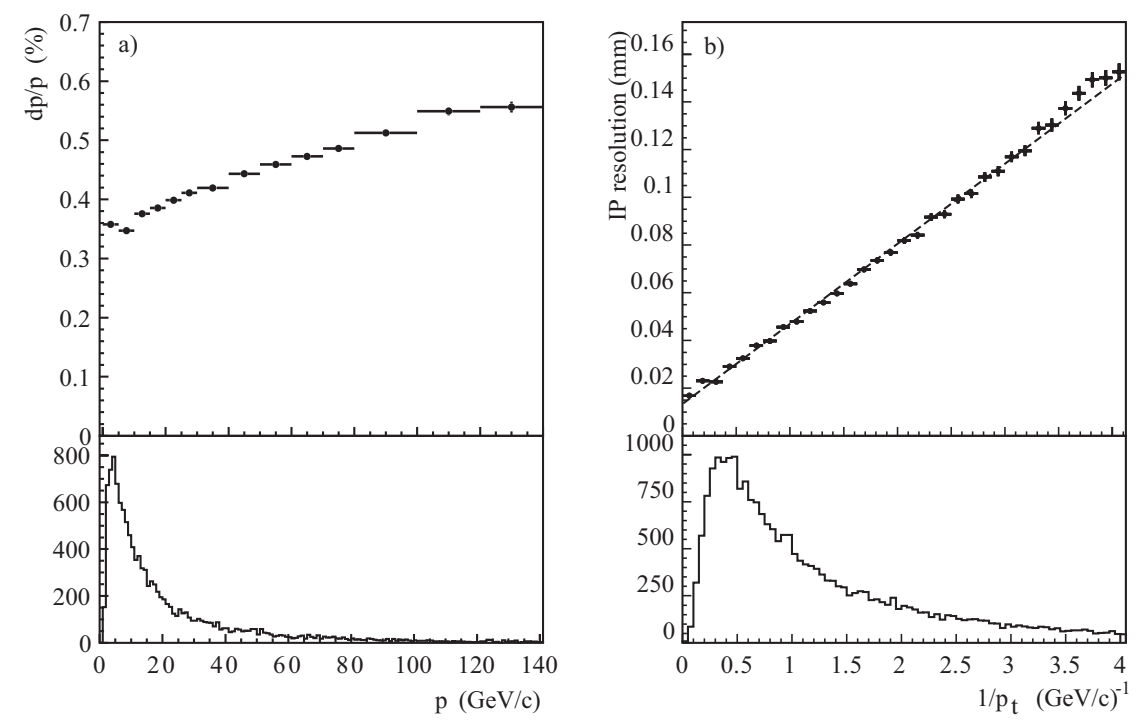

Figure 10.3: Resolution on the reconstructed track parameters at the production vertex of the track: (a) momentum resolution as a function of track momentum, (b) impact parameter resolution, calculated as the sum of the errors in the three projections added in quadrature, as a function of $1 / p_{\mathrm{T}}$. For comparison, the $1 / p_{\mathrm{T}}$ spectra of $\mathrm{B}$ decay particles are shown in the lower part of the plots.

to maintain high efficiency, the reconstruction allows for typically two or three track candidates in the TT to be linked to a single track seed in the T stations.

$\mathrm{K}_{\mathrm{S}}^{0}$ candidates are reconstructed through their decay to $\pi^{+} \pi^{-}$. For $\mathrm{K}_{\mathrm{S}}^{0}$ from $\mathrm{B}^{0} \rightarrow \mathrm{J} / \psi \mathrm{K}_{\mathrm{S}}^{0}$ decays, about $25 \%$ decay inside the active region of the VELO, $50 \%$ decay outside the active region of the VELO but upstream of TT, and the rest decay after TT, and will therefore be difficult to reconstruct. The $\mathrm{K}_{\mathrm{S}}^{0}$ that decay outside the active region of the VELO but before TT are reconstructed using pairs of oppositely charged downstream tracks. The pions from $\mathrm{K}_{\mathrm{S}}^{0}$ that decay within the VELO acceptance give either a long track or an upstream track. The corresponding mass plots are shown in figure 10.4. As can be seen, there exists some combinatorial background from other tracks in the signal events, particularly for the long-upstream category, but this background can be removed by the additional requirements that are imposed when reconstructing the $\mathrm{B}$ meson.

\subsection{Particle identification}

The information from the two RICH detectors, the calorimeters and the muon system is combined for optimal identification of charged particle types $(\mathrm{e}, \mu, \pi, \mathrm{K}, \mathrm{p})$. Photons and neutral pions $\left(\gamma, \pi^{0}\right)$ are identified using the electromagnetic calorimeter, where the $\pi^{0} \rightarrow \gamma \gamma$ decay may be detected as two separate electromagnetic clusters or as a merged cluster. 


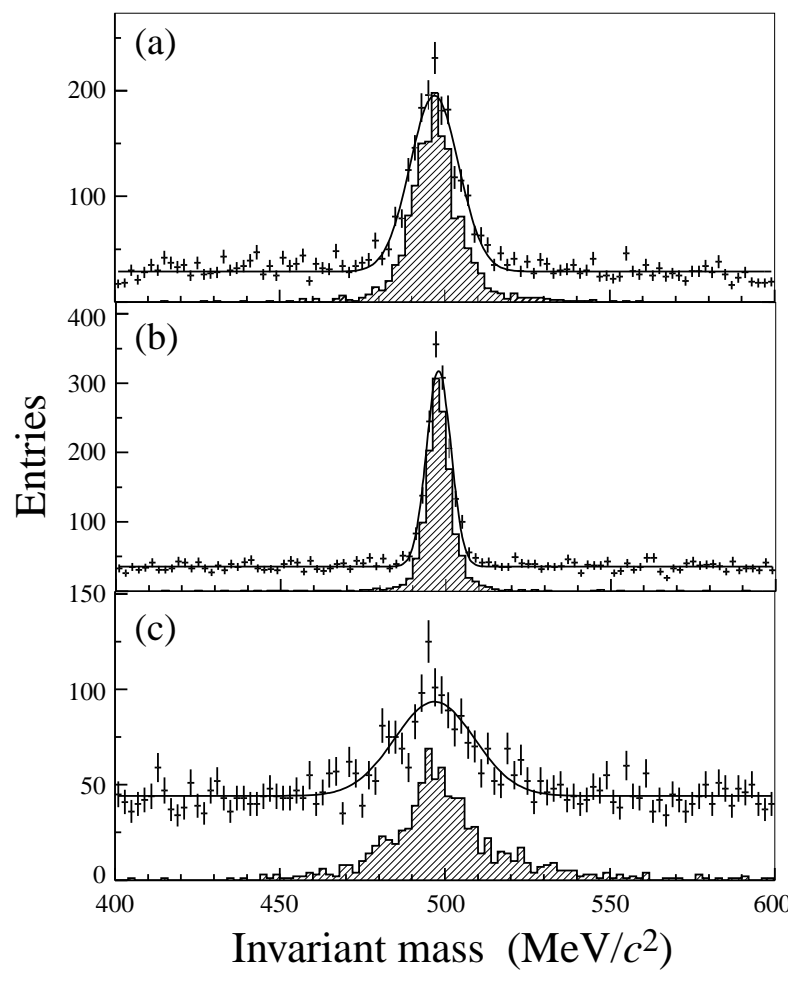

Figure 10.4: Reconstruction of $\mathrm{K}_{\mathrm{S}}^{0} \rightarrow \pi^{+} \pi^{-}$. The $\pi^{+} \pi^{-}$invariant mass is shown in $\mathrm{B}^{0} \rightarrow \mathrm{J} / \psi \mathrm{K}_{\mathrm{S}}^{0}$ signal events, using different categories of tracks for the pion candidates: (a) downstreamdownstream, (b) long-long, (c) long-upstream. Combinations coming from true $\mathrm{K}_{\mathrm{S}}^{0}$ are indicated by the shaded histograms.

\subsubsection{Hadron identification}

Particle identification with the RICH system is performed as follows. The baseline algorithm is based on a log-likelihood approach which matches the observed pattern of hit pixels in the RICH photodetectors to that expected from the reconstructed tracks under a given set of particle hypotheses [224]. In constructing this likelihood it is necessary to calculate the effective emission angle for all pixel-track combinations which could physically be associated through Cherenkov radiation. The likelihood is maximised by varying the particle hypotheses of each track in turn, through electron, muon, pion, kaon and proton. As the likelihood considers all found tracks in the event, and all three radiators simultaneously, the method is referred to as the global pattern-recognition.

The output of the global pattern-recognition is a best hypothesis for each track, and the decrease in log-likelihood when changing from this solution to another hypothesis.

The RICH system provides good particle identification over the entire momentum range. The average efficiency for kaon identification for momenta between 2 and $100 \mathrm{GeV} / c$ is $\varepsilon(\mathrm{K} \rightarrow$ $\mathrm{K}) \sim 95 \%$, with a corresponding average pion misidentification rate $\varepsilon(\pi \rightarrow \mathrm{K}) \sim 5 \%$. Around $30 \mathrm{GeV} / \mathrm{c}$ the identification probability is $\sim 97 \%$ and the misidentification probability $\sim 5 \%$.

Other methods have been investigated to find the Cherenkov rings in the absence of tracking information. These include Markov chain MonteCarlo and Hough Transform techniques [225]. 
Such approaches may be particularly useful in the early stages of data taking, when it may be necessary to study the performance of the RICH detector independently of spectrometer information.

For physics analyses and detector diagnostics it will be very important to understand the performance of the RICH particle identification independently of simulation studies. The dominant $\mathrm{D}^{* \pm} \rightarrow \mathrm{D}^{0}\left(\mathrm{~K}^{-} \pi^{-}\right) \pi^{ \pm}$decay mode will provide a very high statistics unbiased sample of pions and kaons which may be used to measure the RICH performance directly. Studies indicate that with kinematic cuts alone purities of $>95 \%$ can be achieved.

\subsubsection{Muon identification}

Muons are identified by extrapolating well reconstructed tracks with $p>3 \mathrm{GeV} / c$ into the muon stations (particles with $p<3 \mathrm{GeV} / c$ do not reach the muon system). Hits are searched within fields of interest (FOI) around the extrapolation point of the track in each muon station, parameterized as a function of momenta for each station and region. A track is considered as a muon candidate when a minimum number of stations (2-4 depending on momentum) have hits in their corresponding FOI. This number optimizes the resulting efficiency [226].

Using a sample of $\mathrm{B}^{0} \rightarrow \mathrm{J} / \psi \mathrm{K}_{\mathrm{S}}^{0}$ the muon identification efficiency was measured to be $\varepsilon(\mu \rightarrow$ $\mu) \sim 94 \%$ with a corresponding misidentification $\varepsilon(\pi \rightarrow \mu) \sim 3 \%$. The efficiency is a flat function of the momentum above $10 \mathrm{GeV} / c$.

Discriminating variables helping to improve the muon selection purity are constructed from the comparison of slopes in the muon system and the main tracker, and from the average track-hit distance of all hits in FOI associated to the track. For each track the difference in log-likelihood between the muon hypothesis and pion hypothesis is determined, and summed with the values from the RICH and calorimeter systems (if available). By doing this the pion misidentification rate can be reduced to $\sim 1 \%$, whilst maintaining a muon efficiency of $\sim 93 \%$ for muons above $3 \mathrm{GeV} / c$.

The high purity that can be achieved with such cuts is illustrated in figure 10.5 where the $\mu^{+} \mu^{-}$mass plot is shown at the first step in the analysis of $\mathrm{B}_{\mathrm{s}}^{0} \rightarrow \mathrm{J} / \psi \phi$ events, taking all oppositely charged pairs of tracks from signal events that pass the muon identification requirements. The $\mathrm{J} / \psi$ mass peak is reconstructed with a resolution of about $13 \mathrm{MeV} / c^{2}$.

\subsubsection{Electron identification}

The electron identification [227] is mainly based on the balance of track momentum and energy of the charged cluster in the ECAL (figure 10.6), and the matching between the corrected barycenter position of the cluster with the extrapolated track impact point.

A second estimator is related to the bremsstrahlung photons emitted by electrons before the magnet. As there is little material within the magnet, such neutral clusters are expected in a well defined position given by the electron track extrapolation from before the magnet, as illustrated in figure 10.7: if an electron radiates photons when passing through material before the magnet, a cluster with energy $E_{1}$ is seen in the ECAL. If it radiates after the magnet, the bremsstrahlung photon will not lead to a separate cluster. For electron identification the corresponding cluster energy $E_{2}$ will be compared to the track momentum $E_{2}=p$, while the energy of the electron at the origin is $E_{0}=E_{1}+E_{2}$. 


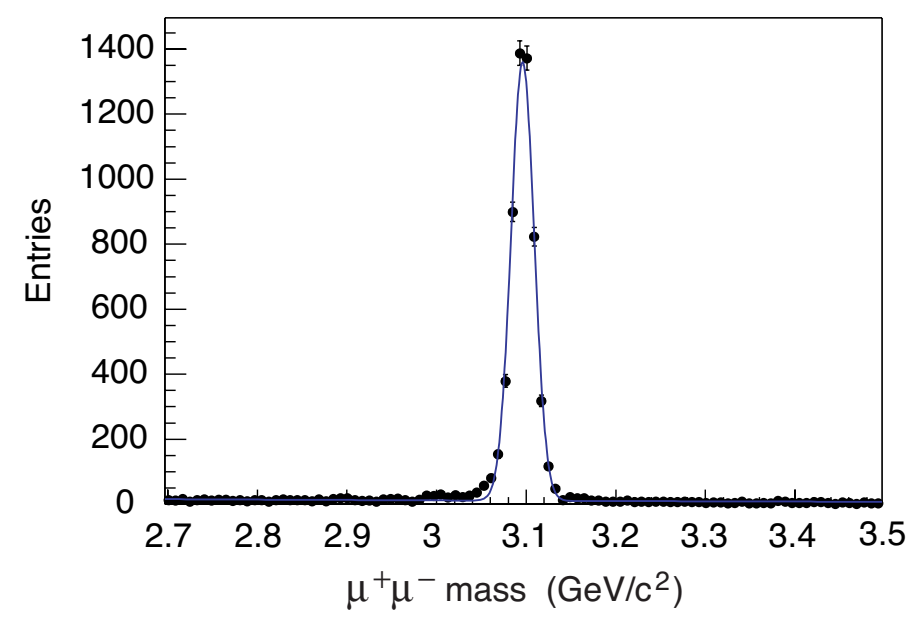

Figure 10.5: Invariant mass plots for the reconstruction of $\mathrm{J} / \psi \rightarrow \mu^{+} \mu^{-}$decays in $\mathrm{B}_{\mathrm{s}}^{0} \rightarrow \mathrm{J} / \psi \phi$ signal events.

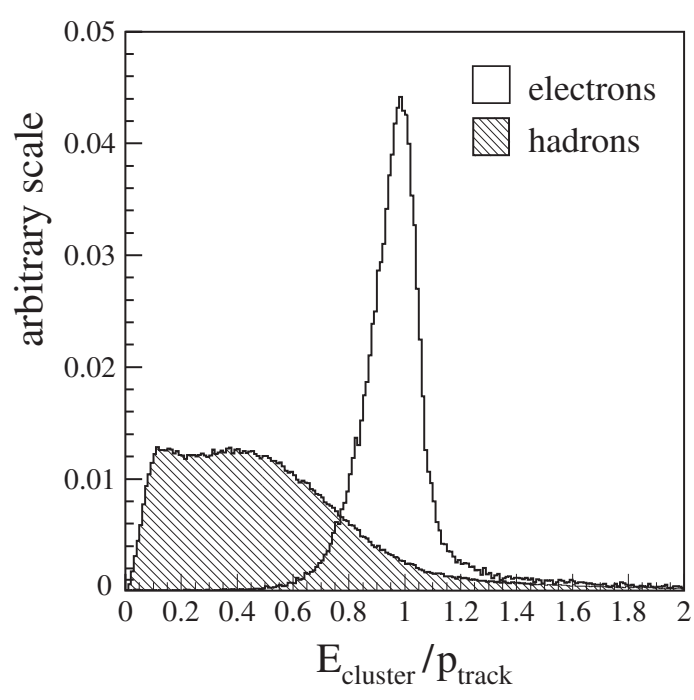

Figure 10.6: The ratio of uncorrected energy of the charged cluster in ECAL to the momentum of reconstructed tracks for electrons (open histogram) and hadrons (shaded histogram).

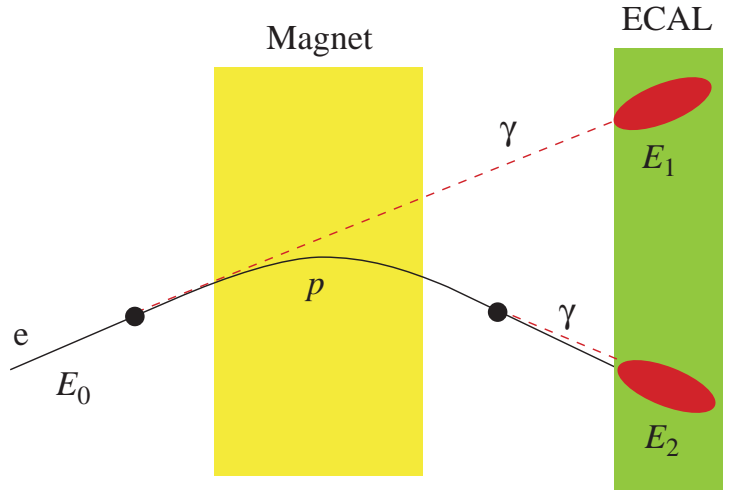

Figure 10.7: Schematic illustration of bremsstrahlung correction.

Further improvement in electron identification is obtained by using the track energy deposition in the preshower detector and the deposition of the energy along the extrapolated particle trajectory in the hadronic calorimeter HCAL.

For particle identification, the calorimeter information is combined with that from the RICH and muon detectors.

To illustrate the performance of electron reconstruction, the $\mathrm{J} / \psi$ mass plot for the decay $\mathrm{J} / \psi \rightarrow \mathrm{e}^{+} \mathrm{e}^{-}$is shown as open points in figure 10.8. The signal is fitted with a function plus a radiative tail, to account for the imperfect correction of bremsstrahlung. The background tracks are 


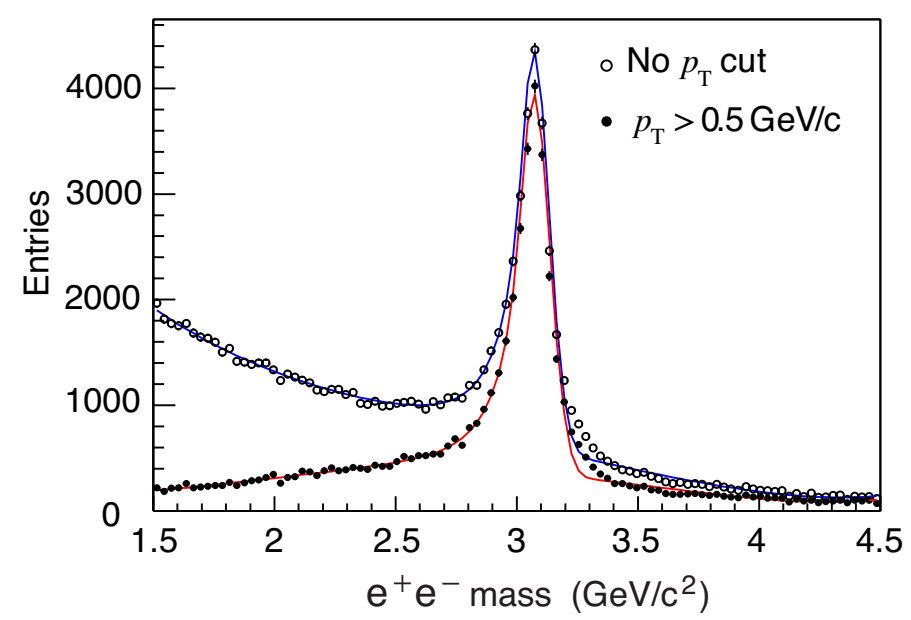

Figure 10.8: Invariant mass plots for the reconstruction of $\mathrm{J} / \psi \rightarrow \mathrm{e}^{+} \mathrm{e}^{-}$decays in $\mathrm{B}_{\mathrm{s}}^{0} \rightarrow \mathrm{J} / \psi \phi$ signal events, where the open points are before any $p_{\mathrm{T}}$ cut, and the solid points are after requiring $p_{\mathrm{T}}>0.5 \mathrm{GeV} / \mathrm{c}$ for the $\mathrm{e}^{ \pm}$candidates.

dominantly of low $p_{\mathrm{T}}$, and can be efficiently rejected by applying the requirement $p_{\mathrm{T}}>0.5 \mathrm{GeV} / \mathrm{c}$ for the electron candidates, as shown by the solid points in figure 10.8 .

The average efficiency to identify electrons from $\mathrm{J} / \psi \rightarrow \mathrm{e}^{+} \mathrm{e}^{-}$decays in $\mathrm{B}^{0} \rightarrow \mathrm{J} / \psi \mathrm{K}_{\mathrm{S}}^{0}$ events in the calorimeter acceptance is $\sim 95 \%$. The pion misidentification fraction for the same events is $0.7 \%$.

\subsubsection{Photon identification}

Photons are reconstructed and identified with the electromagnetic calorimeter, as clusters without an associated track $[121,228]$. The reconstructed tracks are extrapolated to the ECAL face and a cluster-to-track position matching estimator, $\chi_{\gamma}^{2}$, is calculated. Photon candidates correspond to $\chi_{\gamma}^{2}>4$, and are clearly separated from charged particles which form a peak at a small value of $\chi_{\gamma}^{2}$.

The identification of photons converted in the passive material of the apparatus after passing the magnet, e.g. in RICH 2 or in M1, is based on whether there is a hit in the SPD cell that lies in front of central cell of the ECAL cluster. Reconstructed photons from $\mathrm{B}^{0} \rightarrow \mathrm{K}^{*} \gamma$ decays reach the ECAL unconverted in $69 \%$ of cases, while $31 \%$ are converted before the calorimeter. A cut on the energy deposition in the preshower detector can improve the purity of selected samples both for converted and unconverted photons [229].

\subsection{5 $\pi^{0}$ reconstruction}

The neutral pion reconstruction reported in this section is based on the study of $\mathrm{B}^{0} \rightarrow \pi^{+} \pi^{-} \pi^{0}$ decay channel for which the mean transverse momentum of the $\pi^{0}$ is about $3 \mathrm{GeV} / \mathrm{c}$. Below this value the $\pi^{0}$ decays are mostly reconstructed as a resolved pair of well separated photons, while for higher $p_{\mathrm{T}}$ a large fraction of the pairs of photons cannot be resolved as a pair of clusters within the ECAL granularity. About $30 \%$ of the reconstructible $\pi^{0}$ from $\mathrm{B}^{0} \rightarrow \pi^{+} \pi^{-} \pi^{0}$ lead to a single cluster, referred to as a merged $\pi^{0}$. 

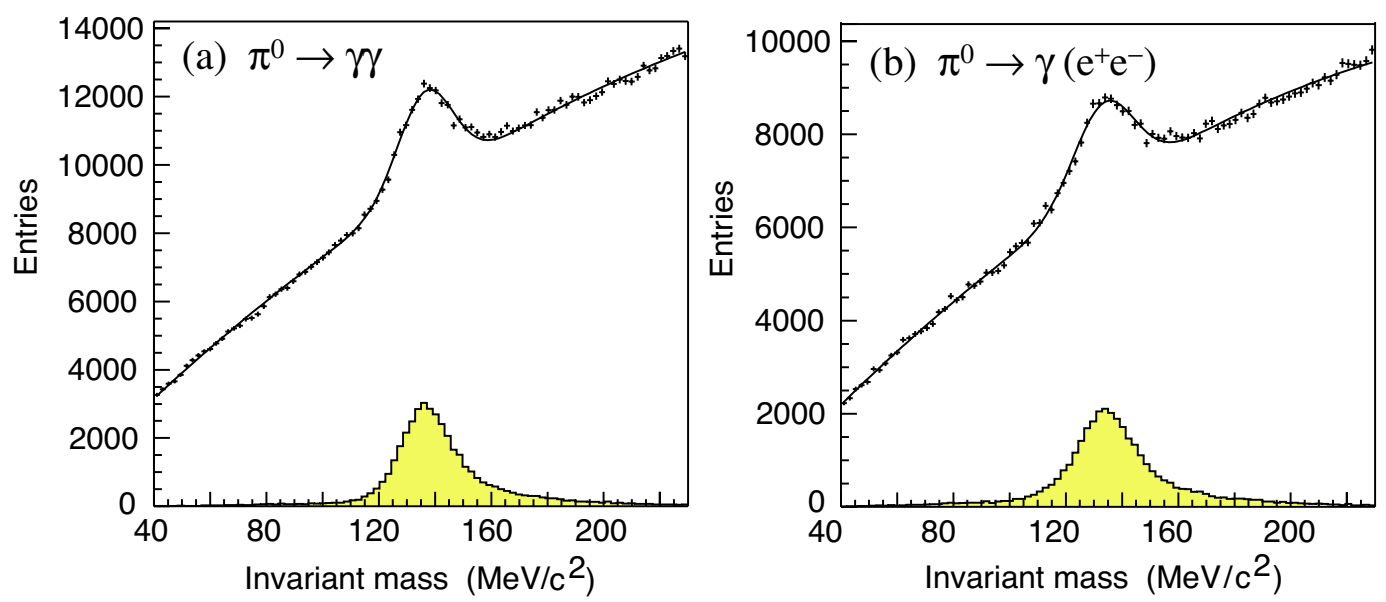

Figure 10.9: $\pi^{0}$ mass distributions, where (a) neither photon has converted, (b) one $\gamma$ converted before the calorimeter. The conversions are identified using the SPD information. The contributions of true $\pi^{0}$ are indicated by the shaded histograms.

Figure 10.9 shows the mass distributions obtained in the case where both photon candidates with $p_{\mathrm{T}}>200 \mathrm{MeV} / \mathrm{c}$ reach the calorimeter (a), and for the case that one photon converted before the calorimeter (b), where the identification of the conversion relies on the SPD signal. The distributions are fitted with the sum of a gaussian and a polynomial function to describe the combinatorial beckground. The fit results in both cases in a mass resolution for the $\pi^{0}$ of $\sim 10 \mathrm{MeV} / \mathrm{c}^{2}$.

An algorithm has been developed to disentangle a potential pair of photons merged into a single cluster. The energy of each cell of the cluster is shared between two virtual sub-clusters according to an iterative procedure based on the expected transverse shape of photon showers. Each of the two sub-clusters is then reconstructed as coming from a photon, as for isolated photons.

The reconstruction efficiency for $\pi^{0}$ that give photons inside the geometrical acceptance is summarized in figure 10.10 for the resolved and merged case.

\subsubsection{Expected global performance}

Given the reconstruction performance described above, the global performance for the reconstruction of B decays in the $\mathrm{LHCb}$ detector is expected to be: a primary vertex resolution of $\sim 10 \mu \mathrm{m}$ transverse to the beam axis and $\sim 60 \mu \mathrm{m}$ along the beam axis; an invariant mass resolution typically in the range between $12 \mathrm{MeV} / \mathrm{c}$ and $25 \mathrm{MeV} / \mathrm{c}$; and a proper lifetime resolution of $\sim 40 \mathrm{fs}$, with a dependence on the decay channel studied. 


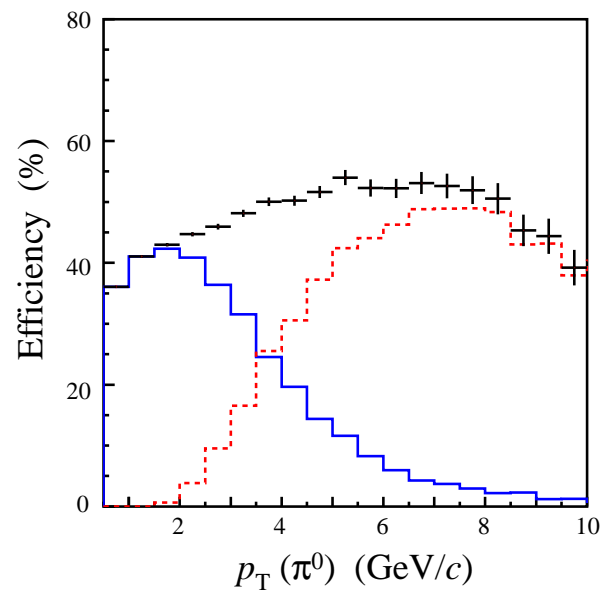

Figure 10.10: The reconstruction efficiency for $\pi^{0}$ decaying into photons inside the geometrical acceptance with $p_{\mathrm{T}}>200 \mathrm{MeV} / \mathrm{c}$, versus the $\pi^{0}$ transverse momentum. The contributions from resolved and merged $\pi^{0}$ reconstruction are indicated by the solid and dashed histograms, respectively. 


\section{Chapter 11}

\section{Summary}

The LHCb experiment has been described as it will be operational at the start up of the LHC, including the detector, its interface to the machine, the hardware-based first level and software-based high level triggers, and the online system. The intrinsic performance of all detector components, as studied in the laboratory and with test-beam measurements, corresponds to expectations. The overall performance for event reconstruction has also been presented, including tracking, vertexing and particle identification, as determined through detailed simulation.

The LHCb experiment will be operational at the time of the very first collisions of the LHC. The first data will be used to measure the real performance of the detector, the trigger behaviour, the reconstruction capabilities and the event selections, and to ensure that the design resolutions have been achieved. This will permit interesting results to be produced in the domain of heavy flavour physics, even with the first period of stable beams in the LHC. The experiment and its associated computing tools will allow a search to be made for the effects of new physics, through high precision measurements of $\mathrm{CP}$ violation and rare decays in the b- and c-physics sectors. 


\section{Acknowledgements}

We are greatly indebted to all CERN departments, without whose support, assistance and active contribution, the preparation of the experimental site, its infrastructure and installation of the $\mathrm{LHCb}$ detector would not have been possible.

We are deeply grateful to our colleagues from the LHC machine for the collaborative relationship and friendly cooperation which enabled running both projects smoothly and effective close to each other.

We acknowledge all the funding agencies which supported the construction and the commissioning of the LHCb detector and also provided the computing infrastructure:

Brazil: CNPq, FINEP and FAPERJ

China: the National Natural Science Foundation of China under contract Nos. 10225522, 10421140657, 10620130426, and the Key Laboratory of Particle and Imaging (Tsinghua University), Ministry of Education of the People's Republic of China

France: CNRS-Institut National de Physique Nucléaire et de Physique des Particules (IN2P3)

Germany: Bundesministerium für Bildung und Forschung and Deutsche Forschungsgemeinschaft Italy: Istituto Nazionale di Fisica Nucleare (INFN)

The Netherlands: Foundation of Fundamental Research of Matter (FOM)

Poland: Ministry of Science and Higher Education

Romania: Ministry of Education and Research

Russia: the Ministry of Science and Technology for the Russian Federation and the Russian Federal Agency for Atomic Energy

Spain: the Spanish MEC (projects FPA2005-06889, FPA2005-06441, FPA2005-07761-C02-01, FPA2005-07761-C02-02, and project CPAN CSD2007-00042 of the Programme ConsoliderIngenio 2010), the "Generalitat de Catalunya" (AGAUR 2005SGR00385) and by the "Xunta de Galicia" (PGIDIT 06PXIC206066PN)

Switzerland: the State Secretariat for Education and Research, the Swiss National Science Foundation and the Ecole Polytechnique Fédérale de Lausanne; the cantons of Vaud and Zuerich

Ukraine: Academy of Sciences of Ukraine

United Kingdom: the Science and Technology Facilities Council (previously PPARC), the Royal Society

United States of America: the U. S. National Science Foundation.

We also acknowledge the European Community support through the projects: HELEN, INTAS, Marie Curie, RII3-CT-2004506078; the regional support from the Region Auvergne (France). 
The construction of the detector would not have been possible without the technical support in each of the participating laboratories: we wish to thank A. Iacoangeli of Università Roma La Sapienza; R. Lunadei and C. Piscitelli of INFN-Roma1; W. Lau, J. Tacon, M. Tacon and S. Yang of Oxford. 


\section{Bibliography}

[1] LHCb collaboration, A Large Hadron Collider beauty experiment, Technical Proposal, CERN-LHCC-98-004, http://cdsweb.cern.ch/record/622031.

[2] LHCb collaboration, R. Antunes-Nobrega et al., LHCb reoptimized detector design and performance, CERN-LHCC-2003-030, http://cdsweb.cern.ch/record/630827.

[3] M. Needham and T. Ruf, Estimation of the material budget of the LHCb detector, Note LHCb-2007-025, http://cdsweb.cern.ch/record/1023537.

[4] J. Christiansen, Requirements to the L0 front-end electronics, Note LHCb-2001-014, http://cdsweb.cern.ch/record/691647.

[5] J. Christiansen, Requirements to the L1 front-end electronics, EDMS document https://edms.cern.ch/document/715154.

[6] P. Moreira et al., G-Link and gigabit ethernet compliant serializer for LHC data transmission, IEEE Nucl. Sci. Symp. Conf. Rec. 2 (2000) 9/6; A radiation tolerant gigabit serializer for LHC data transmission, http://edms.cern.ch/file/906036/1/.

[7] J. Christiansen, Test, time alignment, calibration and monitoring in the LHCb front-end electronics, EDMS document https://edms.cern.ch/document/692583.

[8] B. Taylor, Timing distribution at the LHC, Proceedings of the LECC 2002 Workshop, Colmar France (2002), http://cdsweb.cern.ch/record/592719.

[9] Embedded Local Monitor Board home page, http://elmb.web.cern.ch/ELMB/ELMBhome.html.

[10] G. Haefeli et al., The LHCb DAQ interface board TELL1, Nucl. Instrum. Meth. A 560 (2006) 494.

[11] Radiation hardness assurance, http://lhcb-elec.web.cern.ch/lhcb-elec/html/radiation_hardness.htm.

[12] G. Corti and G. von Holtey, Study of beampipe induced background in the LHCb detector for the optimization of the vacuum chamber design, Note LHCb-2003-085. 
[13] J.R. Knaster, The vacuum chamber in the interaction region of particle colliders: a historical study and developments implemented in the LHCb experiment at CERN, Ph.D. Thesis, CERN-ETSII, Geneva Switzerland (2004).

[14] CERN Safety Instruction IS nr 25, http://safety-commission.web.cern.ch/safetycommission/SC-site/sc_pages/documents/instructions.html

[15] R. Veness et al., Study of minimised UHV flanges for LHC experiments, CERN Vacuum tech. Note EDMS document https://edms.cern.ch/document/350384.

[16] D. Ramos, Design of the fixed beampipe supports inside the acceptance region of the LHCb experiment, CERN Vacuum Tech. Note, EDMS document https://edms.cern.ch/document/882924.

[17] C. Benvenuti et al., Vacuum properties of TiZrV non-evaporable getter films, Vacuum 60 (2001) 57.

[18] O. Grobner, Overview of the LHC vacuum system, Vacuum 60 (2001) 25.

[19] L. Fernandez-Herando et al., The radiation monitoring system for the LHC experimental areas, TS-Note-2004-006, http://cdsweb.cern.ch/record/740728.

[20] B. Todd et al., The architecture, design and realisation of the LHC beam interlock system, in Proceedings of the $10^{\text {th }}$ ICALEPCS International Conference on Accelerator \& Large Experimental Physics Control System, Geneva Switzerland (2005).

[21] E. Effinger et al., Single gain radiation tolerant LHC beam loss acquisition card, in Proceedings of the $8^{\text {th }}$ European Workshop on Beam Diagnostics and Instrumentation for Particle Accelerators, Venice Italy (2007), http://cdsweb.cern.ch/record/1045244.

[22] LHCb collaboration, R. Antunes-Nobrega et al., LHCb computing technical design report, CERN-LHCC-2005-019, http://cdsweb.cern.ch/record/835156.

[23] LHCb collaboration, S. Amato et al., LHCb magnet technical design report, CERN-LHCC-2000-007, http://cdsweb.cern.ch/record/424338.

[24] J. André et al., Status of the LHCb magnet system, IEEE Trans. Appl. Supercond. 12 (2002) 366.

[25] J. André, et al., Status of the LHCb dipole magnet, IEEE Trans. Appl. Supercond. 14 (2004) 509.

[26] M. Patel, M. Losasso and T. Gys., Magnetic shielding studies of the LHCb rich photon detectors, Nucl. Instrum. Meth. A 553 (2005) 114.

[27] F. Bersgma, Calibration of Hall sensors in three dimensions, in Proceedings of the $13^{\text {th }}$ International Magnetic Measurement Workshop (IMMW13), SLAC Stanford, California U.S.A. (2003), http://cdsweb.cern.ch/record/1072471. 
[28] LHCb collaboration, P.R. Barbosa-Marinho et al., Vertex locator technical design report, CERN-LHCC-2001-011, http://cdsweb.cern.ch/record/504321.

[29] P. Koppenburg, Simulation of the vertex trigger preprocessor:effects of noise on L1 performance, Note LHCb-1999-003, http://cdsweb.cern.ch/record/691699.

[30] P. Koppenburg, Effect of pulse overspill on the level 1 trigger, Note LHCb-2001-078, http://cdsweb.cern.ch/record/684442.

[31] D. Petrie, C. Parkes and S. Viret, Study of the impact of VELO misalignments on the LHCb tracking and L1 trigger performance, Note LHCb-2005-056, http://cdsweb.cern.ch/record/899299.

[32] M. Ferro-Luzzi et al., A first study of wake fields in the LHCb detector, Note LHCb-99-041, http://cdsweb.cern.ch/record/684454; Wake fields in the LHCb vertex detector, Note LHCb-99-043, http://cdsweb.cern.ch/record/691632;

$\mathrm{N}$. Van Bakel et al., Wake fields in the LHCb vertex detector: alternative design for the wake fields suppressor, Note LHCb-99-044, http://cdsweb.cern.ch/record/691599.

[33] LHCb VELO GROUP collaboration, Review of the planned replacement of $n$-on- $n$ with $n$-on-p detectors for LHCb-Velo, EDMS document https://edms.cern.ch/document/883223.

[34] S. Löchner and M. Schmelling, The Beetle Reference Manual, Note LHCb-2005-105, http://cdsweb.cern.ch/record/1000429.

[35] R. Mountain et al., VELO module transport box document and operational guide, Syracuse University note, SU-LHCb-2006-02-03.

[36] A. Bates et. al., A facility for long term evaluation and quality assurance of LHCb vertex detector modules, Note LHCb-2007-102, http://cdsweb.cern.ch/record/1061056.

[37] M. Schmelling, Specification of the front-end electronics for the LHCb vertex locator, Note LHCb-2001-048, http://cdsweb.cern.ch/record/691610.

[38] A. Bates et. al., VELO module characterisation: results from the Glasgow LHCb VELO module burn-in, Note LHCb-2007-103, http://cdsweb.cern.ch/record/1046811.

[39] L. Eklund et. al., Radiation tests of the VELO ECS and Analogue Repeater Mezzanines, Note LHCb-2006-001, http://cdsweb.cern.ch/record/926372.

[40] L. Eklund, Control and monitoring of VELO and pile-up level 0 electronics, EDMS document https://edms.cern.ch/document/596194.

[41] D. Breton and D. Charlet, SPECS: the Serial Protocol for the Experiment Control System of LHCb, Note LHCb-2003-004, http://cdsweb.cern.ch/record/681284.

[42] S. Kersten and P. Kind, Technical description of the interlock circuit and system of the ATLAS pixel detector, ATL-IP-ES-0041. 
[43] G. Haefeli, Contribution to the development of the acquisition electronics for the LHCb experiment, LPHE Master thesis, Lausanne Switzerland (2004).

[44] LHCb ST and VELO GROUP collaborations, G. Haefeli and A. Gong, LHCb VELO and ST clusterization on TELL1 EDMS document https://edms.cern.ch/document/690585.

[45] LHCb VELO GROUP, D. Eckstein, VELO raw data format and strip numbering, EDMS document https://edms.cern.ch/document/637676.

[46] LHCb VELO GROUP collaboration, M. Ferro-Luzzi, VELO hardware interlocks, EDMS document https://edms.cern.ch/document/706629.

[47] M. Artuso and J.C. Wang, Study of the spatial resolution achievable with the BTeV pixel sensors, Nucl. Instrum. Meth. A 465 (2000) 115 [hep-ex/ 0007054 ].

[48] C. Parkes and T. Szumlak, VELO event model, Note LHCb-2006-054, http://cdsweb.cern.ch/record/989093.

[49] C. Parkes, T. Ruf and T. Szumlak, Reconstruction of cluster positions in the LHCb VELO, Note LHCb-2007-151, http://cdsweb.cern.ch/record/1074928.

[50] W. Baldini et al., LHCb alignment strategy, Note LHCb-2006-035, http://cdsweb.cern.ch/record/964804.

[51] V. Blobel and C. Kleinwort, A new method for the high-precision alignment of track detectors, Contribution to the Conference on Advanced Statistical Techniques in Particle Physics PHYSTAT2002, Durham U.K., hep-ex/0208021.

[52] T.W. Versloot, Position reconstruction and charge distribution in LHCb VELO silicon sensors, Note LHCb-2007-119, http://cdsweb.cern.ch/record/1073483.

[53] J. Gassner, M. Needham and O. Steinkamp, Layout and expected performance of the LHCb TT station, Note LHCb-2003-140, http://cdsweb.cern.ch/record/728548.

[54] LHCb collaboration, P.R. Barbosa-Marinho et al., LHCb inner tracker technical design report, CERN-LHCC-2002-029, http://cdsweb.cern.ch/record/582793.

[55] J. Gassner, F. Lehner and S. Steiner, The mechanical design of the LHCb silicon trigger tracker, Note LHCb-2004-110, http://cdsweb.cern.ch/record/858499.

[56] J.-L. Agram et al., The silicon sensors for the Compact Muon Solenoid tracker - design and qualification procedure, Note CMS-2003-015, http://cdsweb.cern.ch/record/687875.

[57] A. Bay et al., Hybrid design, procurement and testing for the LHCb silicon tracker, Note LHCb-2005-061, http://cdsweb.cern.ch/record/885752.

[58] I. Abt et al., Gluing silicon with silicone, Nucl. Instrum. Meth. A 411 (1998) 191.

[59] A. Vollhardt, A radiation tolerant fiber-optic readout system for the LHCb silicon tracker, Note LHCb-2005-032, http://cdsweb.cern.ch/record/872267. 
[60] V. Bobillier, J. Christiansen and R. Frei, Grounding, shielding and power distribution in LHCb, Note LHCb-2004-039, http://cdsweb.cern.ch/record/738180.

[61] D. Esperante and A. Vollhardt, Design and development of the control board for the LHCb silicon tracker, Note LHCb-2007-153, http://cdsweb.cern.ch/record/1082457.

[62] TTCrq, http://proj-qpll.web.cern.ch/proj-qpll/ttcrq.htm.

[63] C. Bauer et al., Grounding, shielding and power distribution for the LHCb silicon tracking, Note LHCb-2004-101, http://cdsweb.cern.ch/record/836185.

[64] M. Agari et al., Test beam results of multi-geometry prototype sensors for the LHCb inner tracker, Note LHCb-2002-058, http://cdsweb.cern.ch/record/684437.

[65] R. Bernhard et al., Measurements of prototype ladders for the silicon tracker with laser, Note LHCb-2003-075, http://cdsweb.cern.ch/record/684488.

[66] M. Agari et al., Test-beam measurements on prototype ladders for the LHCb TT station and Inner Tracker, Note LHCb-2003-082, http://cdsweb.cern.ch/record/722699.

[67] J. Gassner et al., Measurements of prototype ladders for the TT station with a laser, Note LHCb-2004-102, http://cdsweb.cern.ch/record/818585.

[68] M. Agari et al., Measurements of a prototype ladder for the TT station in a $120 \mathrm{GeV} / \mathrm{c} \pi^{-}$ beam, Note LHCb-2004-103, http://cdsweb.cern.ch/record/811085.

[69] S. Köstner and U. Straumann, Noise considerations for the beetle amplifier used with long silicon strip detectors, Note LHCb-2005-029, http://cdsweb.cern.ch/record/837194.

[70] M. Needham, Silicon tracker occupancies and clustering, Note LHCb-2007-024, http://cdsweb.cern.ch/record/1023456.

[71] M. Needham and D. Volyanskyy, Updated geometry description for the LHCb trigger tracker, Note LHCb-2006-032, http://cdsweb.cern.ch/record/961216.

[72] A. Perrin and K. Vervink, The inner tracker detector description and its implementation in the XML database, Note LHCb-2006-018, http://cdsweb.cern.ch/record/962061.

[73] LHCb collaboration, P.R. Barbosa-Marinho et al., Outer tracker technical design report, CERN-LHCC-2001-024, http://cdsweb.cern.ch/record/519146.

[74] S. Bachmann, Specifications for the drift gas quality of the outer tracking system, Note LHCb-2002-031; Proposal for the gas distribution in the outer tracking system, Note LHCb-2003-054.

[75] J. Nardulli and N. Tuning, A study of the material in an outer tracker module, Note LHCb-2004-114, http://cdsweb.cern.ch/record/815493.

[76] T. Haas, Aging phenomena in the LHCb outer tracker, Nucl. Instrum. Meth. A 581 (2007) 164. 
[77] A. Berkien et al., The LHCb outer tracker front-end electronics, Note LHCb-2005-025, http://cdsweb.cern.ch/record/1089278;

Y. Guz et al., Study of the global performance of an LHCb OT front-end electronics prototype, Note LHCb-2004-120.

[78] N. Dressnandt et al., Implementation of the ASDBLR and DTMROC ASICS for the ATLAS TRT in DMILL technology, Proceedings of the $6^{\text {th }}$ Workshop on Electronics for LHC Experiments, Cracow Poland (2000), http://cdsweb.cern.ch/record/478863;

$\mathrm{R}$. Bevensee et al., An amplifier-shaper-discriminator with baseline restoration for the ATLAS transition radiation tracker, IEEE Trans. Nucl. Sci. 43 (1996) 1725.

[79] H. Deppe, et al., The OTIS reference manual, Note LHCb-2008-010, http://cdsweb.cern.ch/record/1089277.

[80] U. Stange, Development and characterization of a rad hard readout chip for the LHCb outer tracker detector, PhD Thesis, Heidelberg Germany (2005).

[81] U. Uwer et al., Specifications for the IF 13-2 prototype of the auxiliary board for the outer tracker, Note LHCb-2005-039.

[82] G.W. van Apeldoorn et al., Beam tests of final modules and electronics of the LHCb outer tracker in 2005, Note LHCb-2005-076, http://cdsweb.cern.ch/record/896901.

[83] T. Bauer, J. Nardulli and N. Tuning, Flatness of an outer tracker module, Note LHCb-2005-009.

[84] N. Tuning and A. Pellegrino, Flatness of an outer tracker layer in a prototype C-frame, Note LHCb-2008-003.

[85] H. Dekker et al., The RASNIK/CCD 3-dimensional alignment system, in Proceedings of the $3^{\text {rd }}$ International Workshop On Accelerator Alignment (IWAA 93), Annecy France (1993).

[86] M. Adamus, A. Nawrot and M. Szczekowski, Alignment system for the outer tracker detector in LHCb experiment, Note LHCb-2001-006, http://cdsweb.cern.ch/record/691623; M. Adamus et al., First results from a prototype of the RASNIK alignment system for the outer tracker detector in LHCb experiment, Note LHCb-2002-016.

[87] LHCb RICH GROUP collaboration, N. Brook et al., LHCb RICH 1 engineering design review report, Note LHCb-2004-121, http://cdsweb.cern.ch/record/897981.

[88] F. Metlica, Development of light-weight spherical mirrors for RICH detectors, NIMA 48462, http://dx.doi.org/10.1016/j.nima.2008.07.026.

[89] A. Papanestis, Limits of software compensation for mirror misalignment of the RICH detectors, Note LHCb-2001-141.

[90] R. Plackett, Photon detectors for the Ring Imaging Cherenkov detectors of the LHCb experiment, $\mathrm{PhD}$ Thesis, University of London, London U.K. (2006). 
[91] LHCb collaboration, S. Amato et al., LHCb RICH technical design report, CERN-LHCC-2000-037, http://cdsweb.cern.ch/record/494263.

[92] LHCb RICH GROUP collaboration, M. Adinolfi et al., LHCb RICH 2 engineering design review report, Note LHCb-2002-009, http://cdsweb.cern.ch/record/691478.

[93] T. Bellunato, Development of ring imaging Cherenkov detectors for $\mathrm{LHCb}, \mathrm{PhD}$ Thesis, Università degli Studi di Milano, Milano Italy (2001).

[94] M. Laub, Development of opto-mechanical tools and procedures for a new generation of RICH-detectors at CERN, PhD Thesis, Ceské vysoké ucení technické v Praze, Prague Czech Republic (2001).

[95] L. Fernández Hernando, New automatic techniques to test optical components of the next generation of RICH detectors at CERN, $\mathrm{PhD}$ Thesis, Universitat Politècnica de Catalunya, Barcelona Spain (2001).

[96] G. Aglieri-Rinella, Development of the photon detection, acquisition and optical systems of modern ring imaging Cherenkov detectors, $\mathrm{PhD}$ Thesis, Università degli Studi di Palermo, Palermo Italy (2006).

[97] C. D'Ambrosio et al., The optical systems of LHCb RICHes: a study on the mirror walls and mirrors specifications, Note LHCb-2000-071, http://cdsweb.cern.ch/record/691486.

[98] T. Gys, Magnetic field simulations for the LHCb-RICH 2 detector, Note LHCb-2002-029; M. Patel et al., Magnetic shielding studies of the RICH photon detectors, Note LHCb-2005-055, http://cdsweb.cern.ch/record/920381;

M. Alemi, Passive magnetic shielding calculation for the photodetectors of RICH2, Note LHCb-1998-017, http://cdsweb.cern.ch/record/691679.

[99] M.Y. Barnykov et al., Development of aerogel Cherenkov counters with wavelength shifters and phototubes, Nucl. Instrum. Meth. A 419 (1998) 584.

[100] T. Bellunato et al., Performance of aerogel as Cherenkov radiator, Nucl. Instrum. Meth. A 519 (2004) 493.

[101] T. Bellunato et al., Study of ageing effects in aerogel, Nucl. Instrum. Meth. A 527 (2004) 319.

[102] T. Bellunato et al., Refractive index inhomogeneity within an aerogel block, Nucl. Instrum. Meth. A 556 (2006) 140.

[103] D. Perego, Ageing tests and recovery procedures of silica aerogel, Note LHCb-2008-004.

[104] O. Ullaland, Fluid systems for RICH detectors, Nucl. Instrum. Meth. A 553 (2005) 107.

[105] M. Bosteels et al., LHCb RICH gas system proposal, LHCb-2000-079, http://cdsweb.cern.ch/record/684687. 
[106] A. Braem et al., Metal multi-dielectric mirror coatings for Cherenkov detectors, Nucl. Instrum. Meth. A 553 (2005) 182.

[107] E. Albrecht et al., Operation, optimisation and performance of the DELPHI RICH detectors, Nucl. Instrum. Meth. A 433 (1999) 47.

[108] E. Albrecht et al., The mirror system of COMPASS RICH 1, Nucl. Instrum. Meth. A $\mathbf{5 0 2}$ (2003) 236;

P. Fauland, The COMPASS experiment and the RICH-1 detector, $\mathrm{PhD}$ Thesis, Universität Bielefeld, Mannheim Germany (2004).

[109] M. Alemi et al., First operation of a hybrid photon detector prototype with electrostatic cross-focussing and integrated silicon pixel readout, Nucl. Instrum. Meth. A 449 (2000) 48.

[110] K. Wyllie et al., Silicon detectors and electronics for pixel hybrid photon detectors, Nucl. Instrum. Meth. A 530 (2004) 82.

[111] M. Adinolfi, System test of a three-column LHCb RICH 2 prototype detector, Nucl. Instrum. Meth. A 553 (2005) 328.

[112] P. Moreira et al., A radiation tolerant gigabit serializer for LHC data transmission, Proceedings of the Seventh Workshop on Electronics for LHC Experiments, CERN-LHCC-2001-034, http://cdsweb.cern.ch/record/588665.

[113] J. Christiansen et al., Receiver ASIC for timing, trigger and control distribution in LHC experiments, IEEE Trans. Nucl. Sci 43 (1996) 1773;

TTCrx reference manual V.3.10, http://ttc.web.cern.ch/TTC/TTCrx_manual3.10.pdf, (2005).

[114] C. Arnaboldi et al., The high voltage distribution system for the hybrid photodetector arrays of RICH 1 and RICH 2 at LHCb, IEEE Nucl. Sci. Symp. Conf. Rec. 1 (2005) 413.

[115] C. Gaspar et al, The use of credit card-sized PCs for interfacing electronics boards to the LHCb ECS, Note LHCb-2001-147.

[116] C. D'Ambrosio et al., The LHCb RICH detector control system, Note LHCb-2004-071, http://cdsweb.cern.ch/record/793159.

[117] C. Gaspar and M. Dönszelmann, DIM: a distributed information management system for the DELPHI experiment at CERN, presented at IEEE Conference REAL TIME '93, Vancouver Canada (1993), http://cdsweb.cern.ch/record/254799.

[118] B. Hallgren et al., The Embedded Local Monitor Board (ELMB) in the LHC front-end I/O control system, presented at the $7^{\text {th }}$ Workshop on Electronics for LHC Experiments, Stockholm Sweden (2001), http://cdsweb.cern.ch/record/530675.

[119] M. Adinolfi et al., Performance of the LHCb RICH photodetectors in a charged particle beam, Nucl. Instrum. Meth. A 574 (2007) 39. 
[120] S. Easo et al., Simulation of LHCb RICH detectors using GEANT4, IEEE Trans. Nucl. Sci. 52 (2005) 1665.

[121] LHCb collaboration, S. Amato et al., LHCb calorimeters technical design report, CERN-LHCC-2000-036, http://cdsweb.cern.ch/record/494264.

[122] E. Guschin and S.V. Laptev, Monte-Carlo study of LHCb preshower, Note LHCb-2000-030, http://cdsweb.cern.ch/record/691547.

[123] S. Barsuk et al., Design and construction of the electromagnetic calorimeter for the LHCb experiment, Note LHCb-2000-043, http://cdsweb.cern.ch/record/691508.

[124] R. Djeliadine, O. Iouchtchenko and V.F. Obraztsov, LHCb hadron trigger and HCAL cell size and length optimisation, Note LHCb-1999-035, http://cdsweb.cern.ch/record/691688.

[125] C. Beigbeder-Beau et al., A joint proposal for the level 0 calorimetric triggers, Note LHCb-99-017, http://cdsweb.cern.ch/record/691582.

[126] C. Beigbeder-Beau et al., The front-end electronics for LHCb calorimeters, Note LHCb-2000-028, http://cdsweb.cern.ch/record/691705.

[127] S. Bota et al., Scintillator pad detector front-end electronics, Note LHCb-2000-027, http://cdsweb.cern.ch/record/691544.

[128] G. Böhner et al., Front-end electronics for the LHCb preshower detector, Note LHCb-2000-048, http://cdsweb.cern.ch/record/691511.

[129] B. Delcourt, J. Lefrançois, Investigation of widening of the $\pi^{0}$ mass peak with electronic defects, Note LHCb-2000-029, http://cdsweb.cern.ch/record/691719.

[130] S.N. Filippov et al., Design and construction of the LHCb scintillator-pad/preshower detector, Note LHCb-2000-042, http://cdsweb.cern.ch/record/691521.

[131] S.N. Filippov et al., Experimental performance of PS/SPD prototypes, Note LHCb-2000-031, http://cdsweb.cern.ch/record/691545.

[132] G. Böhner et al., Very front-end electronics for LHCb preshower, Note LHCb-2000-047, http://cdsweb.cern.ch/record/691512.

[133] Z. Ajaltouni et al., Study of multianode photomultipliers for the electromagnetic calorimeter preshower read out of the LHCb experiment, Nucl. Instrum. Meth. A 504 (2003) 9; Photomultiplier pulse read out system for the preshower detector of the LHCb experiment, Nucl. Instrum. Meth. A 504 (2003) 250.

[134] E. Aguiló et al., Test of multi-anode photomultiplier tubes for the LHCb scintillator pad detector, Nucl. Instrum. Meth. A 538 (2005) 255.

[135] E. Graugés et al., Mass characterization of MaPMT tubes for the LHCb scintillator pad detector, Nucl. Instrum. Meth. A 572 (2007) 427. 
[136] S. Monteil, Photodetector performance for the LHCb pre-shower detector, in Proceedings of the $11^{\text {th }}$ International Conference on Calorimetry in High-Energy Physics, CALOR 2004, Perugia Italy (2004).

[137] L. Garrido et al., Results of tagged photon test beam for the scintillator pad detector, Note LHCb-2000-032, http://cdsweb.cern.ch/record/691546;

E. Aguiló et al., Backsplash testbeam results for the SPD subdetector of LHCb, Nucl. Instrum. Meth. A 546 (2005) 438.

[138] HERA-B collaboration, HERA-B: an experiment to study CP violation in the $B$ system using an internal target at the HERA proton ring. Proposal, DESY-PRC-94-002; HERA-B: an experiment to study CP violation in the $B$ system using an internal target at the HERA proton ring. Design report, DESY-PRC-95-01.

[139] PHENIX collaboration, PHENIX: preliminary conceptual design report, BNL-PROPOSAL-R2.

[140] HERA-B collaboration, E. Tarkovsky, The HERA-B electromagnetic calorimeter, Nucl. Instrum. Meth. A 379 (1996) 515.

[141] A. Bazilevsky et al., Performance of the PHENIX EM calorimeter, IEEE Trans. Nucl. Sci. 43 (1996) 1491.

[142] J. Badier et al., Shashlik calorimeter beam-test results, Nucl. Instrum. Meth. A 348 (1994) 74.

[143] A. Arefiev et al., Design, construction, quality control and performance study with cosmic rays of modules for the LHCb electromagnetic calorimeter, Note LHCb-2007-148, http://cdsweb.cern.ch/record/1080559.

[144] A. Arefiev et al., Beam test results of the LHCb electromagnetic calorimeter, Note LHCb-2007-149, http://cdsweb.cern.ch/record/1103500.

[145] V. Brekhovskikh et al., The WLS fiber time properties study, Note LHCb-2000-039, http://cdsweb.cern.ch/record/691514.

[146] R.I. Dzhelyadin, The LHCb hadron calorimeter, Nucl. Instrum. Meth. A 494 (2002) 332, also in Proceedings of the $8^{\text {th }}$ International Conference on Instrumentation for Colliding Beam Physics, Novosibirsk Russia (2002).

[147] L.G. Afanasieva et al., The hadron calorimeter design and construction, Note LHCb-2000-045, http://cdsweb.cern.ch/record/691506.

[148] M. Bonnet et al., The hadron calorimeter prototype design and construction, Note LHCb-2000-035, http://cdsweb.cern.ch/record/691513.

[149] C. Coca et al., The hadron calorimeter prototype beam-test results, Note LHCb-2000-036, http://cdsweb.cern.ch/record/691519. 
[150] R.I. Dzhelyadin et al., The hadron calorimeter module-0 construction, Note LHCb-2001-122.

[151] G.I. Britvich et al., The HCAL optics radiation damage study, Note LHCb-2000-037, http://cdsweb.cern.ch/record/691516.

[152] I. Korolko, J. Ocariz and A. Schopper, HCAL performance with irradiated sub-components, Note LHCb-2000-038, http://cdsweb.cern.ch/record/691524.

[153] C. Beigbeder-Beau et al., The readout of the LHCb calorimeters, Note LHCb-2000-046, http://cdsweb.cern.ch/record/691493.

[154] N. Dumont-Dayot, The preprocessor FPGA for the ECAL/HCAL and PS/SPD detectors, LAPP EDMS I-008689 https://edms.in2p3.fr/file/I-008689/1/pp-fpga-firmware.pdf.

[155] D. Boget et al., The readout of the LHCb calorimeters, EDMS 527942 http://edms.cern.ch/document/527942.

[156] G. Böhner, LHCb preshower signal characteristics, Note LHCb-2000-026, http://cdsweb.cern.ch/record/691597.

[157] D. Gascón et al, The front-end electronics of the scintillator pad detector of LHCb calorimeter, in Proceedings of the $12^{\text {th }}$ LECC Workshop, Valencia Spain (2006), http://cdsweb.cern.ch/record/1027429.

[158] D. Gascón et al., A BICMOS synchronous pulse discriminator for the LHCb calorimeter system, in Proceedings of the $8^{\text {th }}$ LECC Workshop, Colmar France (2002), http://cdsweb.cern.ch/record/619291.

[159] S. Luengo et al., SPD very front end electronics, Nucl. Instrum. Meth. A 567 (2006) 310.

[160] D. Breton and D. Charlet, Using the SPECS in LHCb, Note LHCb-2003-005.

[161] A. Arefiev et al., Design of PMT base for the LHCb electromagnetic calorimeter, Note LHCb-2003-150.

[162] G. Avoni et al., The HERA-B ECAL electronics and monitoring, in Proceedings of International Conference on Calorimetry in Particle Physics, CALOR 2000, Annecy France (2000).

[163] S. Amato et al., Analysis of the $\mathrm{B}_{\mathrm{s}}^{0} \rightarrow \mu^{+} \mu^{-}$decay with the reoptimized LHCb detector, Note LHCb-2003-165, http://cdsweb.cern.ch/record/726431.

[164] LHCb collaboration, LHCb muon system technical design report, CERN-LHCC-2001-010, http://cdsweb.cern.ch/record/504326.

[165] LHCb collaboration, LHCb addendum to the muon system technical design report, CERN-LHCC-2003-002, http://cdsweb.cern.ch/record/600536. 
[166] LHCb collaboration, $L H C b$ second addendum to the muon system technical design report, CERN-LHCC-2005-012, http://cdsweb.cern.ch/record/831955.

[167] G. Martellotti, R. Santacesaria and A. Satta, Muon system digitization, Note LHCb-2004-063, http://cdsweb.cern.ch/record/784561.

[168] R. Santacesaria and A. Satta, A new calculation of the low energy background in the muon system, Note LHCb-2003-057, http://cdsweb.cern.ch/record/684464.

[169] G. Martellotti, R. Santacesaria and A. Satta, Particle rates in the LHCb muon detector, Note LHCb-2005-075, http://cdsweb.cern.ch/record/896904.

[170] B. Bochin et al., Wire pad chamber for LHCb muon system, Note LHCb-2000-003, http://cdsweb.cern.ch/record/681334.

[171] B. Bochin et al., Beam tests of WPC-7 prototype of wire pad chambers for the LHCb muon system, Note LHCb-2000-102, http://cdsweb.cern.ch/record/691718.

[172] D. Hutchcroft et al., Results obtained with the first four gap MWPC prototype chamber, Note LHCb-2001-024, http://cdsweb.cern.ch/record/691631.

[173] B. Bochin et al., Test results of a full size prototype of the muon chambers for region M2/R4 of the LHCb muon system, Note LHCb-2002-025, http://cdsweb.cern.ch/record/681216.

[174] B. Maréchal et al., Construction and test of the prototype chamber for region 1 of the LHCb muon station 2, Note LHCb-2002-034, http://cdsweb.cern.ch/record/691482.

[175] M. Anelli et al., Test of MWPC prototypes for R3 of the LHCb muon system, Note LHCb-2004-074, http://cdsweb.cern.ch/record/793160.

[176] G. Lanfranchi, Time resolution and aging properties of the MWPCs for the LHCb muon system, Nucl. Instrum. Meth. A 535 (2004) 221.

[177] E. Danè et al., Detailed study of the gain of the MWPCs for the LHCb muon system, Nucl. Instrum. Meth. A 572 (2007) 682.

[178] A. Kachtchouk et al., Performance study of a MWPC prototype for the LHCb muon system with the ASDQ chip, Note LHCb-2000-062, http://cdsweb.cern.ch/record/681340.

[179] W. Riegler, Detector physics and performance: simulation of the MWPCs for the full LHCb muon system, Note LHCb-2000-060, http://cdsweb.cern.ch/record/681186.

[180] R. Veenhof, Garfield - simulation of gaseous detectors, http://consult.cern.ch/writeup/garfield/.

[181] I. Smirnov, Heed: interactions of particles with gases, http://consult.cern.ch/writeup/heed/.

[182] S. Biagi, Magboltz: transport of electrons in gas mixtures, http://consult.cern.ch/writeup/magboltz/. 
[183] A. Kachtchouk et al., Design and construction of the wire chambers for the LHCb muon system, Note LHCb-2001-026, http://cdsweb.cern.ch/record/684460.

[184] M. Anelli et al., Quality tests of the LHCb muon chambers at the LNF production site, IEEE Trans. Nucl. Sci. 53 (2006) 330.

[185] A.F. Barbosa et al., Production and quality control of MWPC for the LHCb muon system at CERN, IEEE Trans. Nucl. Sci. 53 (2006) 336.

[186] E. Dané, D. Pinci and A. Sarti, Results of the quality controls of the four-gap MWPCs produced at LNF for LHCb, IEEE Trans. Nucl. Sci. 54 (2007) 354.

[187] P. Ciambrone et al., Automated wire tension measurement system for LHCb muon chambers, Nucl. Instrum. Meth. A 545 (2005) 156.

[188] W. Baldini et al., A laser based instrument for MWPC wire tension measurement, Note LHCb-2007-120, http://cdsweb.cern.ch/record/1055333.

[189] A. Alves et al., Results of the MWPC gas gain uniformity test performed at CERN, Note LHCb-2007-115, http://cdsweb.cern.ch/record/1054084.

[190] S. Agosteo et al., A facility for the test of large-area muon chambers at high rates, Nucl. Instrum. Meth. A 452 (2000) 94.

[191] V. Souvorov et al., First results of an aging test of a full scale MWPC prototype for the LHCb muon system, Nucl. Instrum. Meth. A 515 (2003) 220.

[192] M. Anelli et al., Extensive ageing test of two prototypes of four-gap MWPC for the LHCb muon system, Note LHCb-2004-029, http://cdsweb.cern.ch/record/733605.

[193] F. Sauli, GEM: a new concept for electron amplification in gas detectors, Nucl. Instrum. Meth. A 386 (1997) 531.

[194] G. Bencivenni et al., Advances in triple-GEM detector operation for high-rate particle triggering, Nucl. Instrum. Meth. A 513 (2003) 264.

[195] M. Alfonsi et al., Advances in fast multi-GEM-based detector operation for high-rate charged-particle triggering, IEEE Trans. Nucl. Sci. 51 (2004) 2135.

[196] A. Bressan et al., Beam tests of the gas electron multiplier, Nucl. Instrum. Meth. A 425 (1999) 262.

[197] M. Alfonsi et al., Studies of etching effects on triple-GEM detectors operated with CF/sub 4/-based gas mixtures, IEEE Trans. Nucl. Sci. 52 (2005) 2872.

[198] M. Alfonsi et al., Aging measurements on triple-GEM detectors operated with $\mathrm{CF}_{4}$ based gas mixtures, Nucl. Phys. B 150 (Proc. Suppl.) (2006) 159.

[199] W. Bonivento, D. Marras and G. Auriemma, Production of the front-end boards of the LHCb muon system, Note LHCb-2007-150, http://cdsweb.cern.ch/record/1079951. 
[200] D. Moraes et al., CARIOCA $0.25 \mu \mathrm{m}$ CMOS fast binary front-end for sensor interface using a novel current-mode feedback technique, , IEEE Proc. Int. Symp. Circuits Syst. 1 (2001) 360 .

[201] W. Bonivento et al., Status of the CARIOCA project, in Proceedings of the $7^{\text {th }}$ workshop on electronics for LHC experiments, Stockholm Sweden (2001), http://lhc-electronicsworkshop.web.cern.ch/LHC-electronics-workshop/2001/muon/moraesboniv.pdf.

[202] W. Bonivento, Design and performance of the front-end electronics of the LHCb muon detector, in Proceedings of the $11^{\text {th }}$ workshop on electronics for LHC and future experiments, Heidelberg Germany (2005), http://www.lecc2005.uni-hd.de/.

[203] S. Cadeddu, C. Deplano and A. Lai, The DIALOG chip in the front-end electronics of the LHCb muon detector, IEEE Trans. Nucl. Sci. 52 (2005) 2726.

[204] S. Cadeddu et al., DIALOG and SYNC: a VLSI chip set for timing of the LHCb muon detector, IEEE Trans. Nucl. Sci. 51 (2004) 1961.

[205] V. Bocci et al., The services boards system for the LHCb muon detector (equalization, timing and monitoring of the $120 \mathrm{k}$ front end channels in the LHCb muon detector), IEEE Nucl. Sci. Symp. Conf. Rec. 3 (2007) 2134.

[206] Technical documentation on the ELMB can be found at Embedded Local Monitor Board home page, http://elmb.web.cern.ch/ELMB/ELMBhome.html.

[207] G. Felici et al., The L0 Off Detector Electronics (ODE) for the LHCb muon spectrometer, in Proceedings of the $10^{\text {th }}$ Workshop on Electronics for LHC and future experiments, Boston U.S.A. (2004), http://lhc-workshop-2004.web.cern.ch/lhc-workshop-2004/.

[208] P. Moreira, QPLL manual v. 1.1, http://proj-qpll.web.cern.ch/proj-qpll/images/qpllManual.pdf, (2005).

[209] P. Moreira et al., A radiation tolerant gigabit serializer for LHC data transmission, in Proceedings of the $7^{\text {th }}$ Workshop on Electronics for LHC Experiments, Stockholm Sweden (2001), http://lhc-electronics-workshop.web.cern.ch/LHC-electronicsworkshop/2001/opto/Moreira.pdf.

[210] G. Corradi et al., A novel high-voltage system for a triple GEM detector, Nucl. Instrum. Meth. A 572 (2007) 96.

[211] G. Sabatino et al., Cluster size measurements for the LHCb muon system M5R4 MWPCs using cosmic rays, Note LHCb-2006-011, http://cdsweb.cern.ch/record/939097.

[212] S. de Capua et al., Study of gas gain uniformity for the LHCb muon system MWPCs using cosmic rays, Note LHCb-2006-010, http://cdsweb.cern.ch/record/939085.

[213] M. Anelli et al., Test of a MPWC for the LHCb muon system at the GIF at CERN, Note LHCb-2005-003, http://cdsweb.cern.ch/record/815058. 
[214] LHCb collaboration, R. Antunes-Nobrega et al., LHCb trigger system technical design report, CERN-LHCC-2003-031, http://cdsweb.cern.ch/record/630828.

[215] E. Aslanides et al., The level-0 muon trigger for the LHCb experiment, Nucl. Instrum. Meth. A 579 (2007) 989.

[216] M. Krasowski et al., Primary vertex reconstruction, Note LHCb-2007-011, http://cdsweb.cern.ch/record/1057577.

[217] LHCb collaboration, P.R. Barbosa-Marinho et al., LHCb online system technical design report, CERN-LHCC-2001-040, http://cdsweb.cern.ch/record/545306.

[218] LHCb collaboration, Addendum to the LHCb online system technical design report, CERN-LHCC-2005-039, http://cdsweb.cern.ch/record/903611.

[219] RD12 collaboration, Timing, Trigger and Control (TTC) systems for the LHC, http://ttc.web.cern.ch/TTC/ and links therein.

[220] T. Sjöstrand et al., High-energy physics event generation with PYTHIA 6.1, Comput. Phys. Commun. 135 (2001) 238 [hep-ph/ 0010017 ].

[221] GEANT detector description and simulation tool, CERN Program Library long writeup W5013 (1994).

[222] J. van Tilburg, Matching VELO tracks with seeding tracks, Note LHCb-2001-103, http://cdsweb.cern.ch/record/691686;

R. Forty, Track seeding, Note LHCb-2001-109, http://cdsweb.cern.ch/record/691473;

R. Hierck, Track following in LHCb, Note LHCb-2001-112, http://cdsweb.cern.ch/record/691752;

M. Benayoun and O. Callot, The forward tracking, an optical model method, Note LHCb-2002-008, http://cdsweb.cern.ch/record/684710;

Y. Xie, Short track reconstruction with VELO and TT, Note LHCb-2003-100, http://cdsweb.cern.ch/record/684462;

O. Callot, M. Kucharczyk and M. Witek, VELO-TT track reconstruction, Note LHCb-2007-010, http://cdsweb.cern.ch/record/1027834;

D. Hutchcroft et al., VELO pattern recognition, Note LHCb-2007-013, http://cdsweb.cern.ch/record/1023540;

O. Callot and S. Hansmann-Menzemer, Performance of the forward tracking, Note LHCb-2007-015, http://cdsweb.cern.ch/record/1033584;

R. Forty and M. Needham, Updated performance of the T-seeding, Note LHCb-2007-023, http://cdsweb.cern.ch/record/1023581;

O. Callot, Downstream pattern recognition, Note LHCb-2007-026,

http://cdsweb.cern.ch/record/1025827;

M. Needham, Performance of the track matching, Note LHCb-2007-129, http://cdsweb.cern.ch/record/1060807; Performance of the LHCb track reconstruction software, Note LHCb-2007-144, http://cdsweb.cern.ch/record/1080556. 
[223] R. Hierck et al., Performance of the LHCb OO track-fitting software, Note LHCb-2000-086, http://cdsweb.cern.ch/record/684697;

E. Rodrigues, The LHCb track Kalman fit, Note LHCb-2007-014;

E. Bos et al., The trajectory model for track fitting and alignment, Note LHCb-2007-008, http://cdsweb.cern.ch/record/1025826.

[224] R. Forty and O. Schneider, RICH pattern recognition, Note LHCb-98-040, http://cdsweb.cern.ch/record/684714.

[225] R. Muresan, Cherenkov ring reconstruction methods, Note LHCb-2007-121, http://cdsweb.cern.ch/record/1057872.

[226] J.R.T. de Mello Neto and M. Gandelman, Muon ID performance with the reoptimized LHCb detector, Note LHCb-2003-089, http://cdsweb.cern.ch/record/691744; M. Gandelman and E. Polycarpo, The performance of the LHCb muon identification procedure, Note LHCb-2007-145, http://cdsweb.cern.ch/record/1093941.

[227] H. Terrier and I. Belyaev, Particle identification with LHCb calorimeters, Note LHCb-2003-092, http://cdsweb.cern.ch/record/691743.

[228] V. Breton, N. Brun and P. Perret, A clustering algorithm for the LHCb electromagnetic calorimeter using cellular automaton, Note LHCb-2001-123, http://cdsweb.cern.ch/record/681262.

[229] O. Deschamps et al., Photon and neutral pion reconstruction, Note LHCb-2003-091, http://cdsweb.cern.ch/record/691634. 